\title{
Molecular mechanisms of lipid-rich myelin membrane sheet formation
}

\author{
Dissertation \\ in partial fulfilment of the requirements \\ for the degree "Doctor rerum naturalium" \\ in the Molecular Biology Program at the \\ Georg August University Göttingen, Faculty of Biology
}

submitted by

Shweta Aggarwal

born in

Jalandhar, India

Göttingen 2012 
Members of the Thesis Committee:

Prof. Dr. Mikael Simons, Reviewer

Max Planck Institute of Experimental Medicine

Department of Neurology, University of Göttingen

Prof. Dr. Peter Rehling, Reviewer

Department of Biochemistry, University of Göttingen

Prof. Dr. Dirk Görlich

Max Planck Institute for Biophysical Chemistry

Date of the oral examination: $\quad 23^{\text {rd }}$ October, 2012 


\section{Affidavit}

I hereby declare that this $\mathrm{PhD}$ thesis "Molecular mechanisms of lipid-rich myelin membrane sheet formation" has been written independently with no other aids or sources than quoted.

Shweta Aggarwal

September, 2012

Göttingen, Germany 



\section{Publications}

Shweta Aggarwal, Larisa Yurlova, Nicolas Snaidero, Christina Reetz, Steffen Frey, Johannes Zimmermann, Gesa Pähler, Andreas Janshoff, Jens Friedrichs, Daniel J. Müller, Cornelia Goebel, Mikael Simons.

A size barrier limits protein diffusion at the cell surface to generate lipid-rich myelinmembrane sheets.

Developmental Cell, 21, 445-456, September 2011.

Shweta Aggarwal, Larisa Yurlova, Mikael Simons.

Central nervous system myelin: structure, synthesis and assembly.

Trends in Cell Biology, 21(10), 585-593, October 2011.

Larisa Yurlova, Nicoletta Kahya, Shweta Aggarwal, Hermann-Josef Kaiser, Salvatore Chiantia, Mostafa Bakhti, Yael Pewzner-Jung, Oshrit Ben-David, Anthony H. Futerman, Britta Brügger, Mikael Simons.

Self-seggregation of myelin membrane lipids in model membranes Biophysical Journal, 101, 2713-2720, December 2011.

Mikael Simons, Nicolas Snaidero, Shweta Aggarwal.

Cell polarity in myelinating glia: From membrane flow to diffusion barriers

Biochimica et Biophysica Acta, 1821, 1146-1153, January 2012.

Shweta Aggarwal, Nicolas Snaidero, Steffen Frey, Gesa Pähler, Andreas Janshoff, Paula Sánchez, Marie-Theres Weil, Iwan Schaap, Dirk Görlich, Mikael Simons.

Functional self-assembly by phase separation in myelin biogenesis Manuscript in preparation. 



\section{Contents}

Publications $\quad$ iv

Contents vii

List of Figures . . . . . . . . . . . . . . . . . . . . . xi

List of Tables . . . . . . . . . . . . . . . . . . . . . . xv

Abbreviations $\quad$ xvii

$\begin{array}{ll}\text { Abstract } & \mathrm{xxi}\end{array}$

1 Introduction 1

1.1 Myelin . . . . . . . . . . . . . . . . . . . . . . 1

1.2 Oligodendrocyte differentiation . . . . . . . . . . . . . . . 1

1.3 Myelin architecture . . . . . . . . . . . . . . . . . 2

1.4 Myelin lipid composition . . . . . . . . . . . . . . . . . . 3

1.5 Myelin protein composition . . . . . . . . . . . . . 5

1.5.1 Proteolipid protein . . . . . . . . . . . . . . 5

1.5.2 Myelin basic protein (MBP) . . . . . . . . . . . . . 7

1.5.3 Other myelin proteins . . . . . . . . . . . . . . . 12

1.6 Myelin Domains . . . . . . . . . . . . . . . . . . . . . . . . . 14

1.7 Cellular polarization . . . . . . . . . . . . . . . . . 17

1.7.1 Mechanisms of cellular polarization . . . . . . . . . . 17

1.7.2 Mechanisms of myelin membrane polarization . . . . . . . . 21

1.8 Aims of the work . . . . . . . . . . . . . . . . . . . . 22

2 Materials and Methods $\quad 23$ 


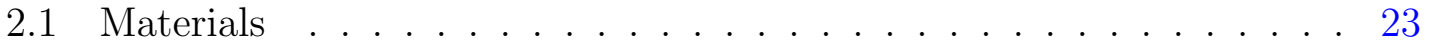

2.1.1 Antibodies . . . . . . . . . . . . . . . 23

2.1.2 Chemicals, Enzymes and Kits . . . . . . . . . . . . . . . 24

2.1.3 Mammalian cell lines and bacterial strains . . . . . . . . . . 26

2.2 Methods . . . . . . . . . . . . . . . . . . . . 27

2.2.1 Molecular biology and biochemical methods . . . . . . . . . 27

2.2.2 Expression and purification of proteins . . . . . . . . . . . 29

2.2 .3 Biochemical assays . . . . . . . . . . . . . . 30

2.2.4 Cell culture . . . . . . . . . . . . . . . . . 33

2.2.5 Transient Transfection . . . . . . . . . . . . . . . . 35

2.2 .6 Immunocytochemistry . . . . . . . . . . . . . 36

2.2 .7 Microscopy . . . . . . . . . . . . . 36

2.2.8 Biomimetic SLB-MBP-GUV assay . . . . . . . . . . . . . . 39

2.2 .9 MBP aggregation assay . . . . . . . . . . . . . 40

2.2 .10 Image Analysis . . . . . . . . . . . . . . . . . . 41

2.2.10.1 Statistical Analysis . . . . . . . . . . . . . . . . 42

3 Results 43

3.1 MBP generates lipid-rich myelin membrane sheets . . . . . . . . . . . 43

3.1.1 Cellular model of cultured oligodendrocytes . . . . . . . . . 43

3.1.2 MBP regulates surface polarity in oligodendrocytes . . . . . . 45

3.1.3 Cytoplasmic size-based diffusion of proteins into the myelin membrane sheets . . . . . . . . . . . . . 50

3.1.4 Diffusion barrier characterization with FRAP . . . . . . . . 52

3.1.5 MBP regulates lipid/protein ratios in myelin . . . . . . . . . 55

3.1.6 In vivo validation of size barrier hypothesis . . . . . . . . . . . 57

3.1.7 Estimation of MBP amounts in primary oligodendrocytes . . . 60

3.1.8 A biomimetic assay and barrier reconstitution . . . . . . . . . 61

3.2 Sieving function of MBP requires molecular self-assembly . . . . . . . 65

3.2.1 Displacement of bulky proteins from the myelin membrane sheets .......................... 65

3.2.2 Reconstitution of MBP barrier in a fibroblast cell line . . . . . 67 
3.2.3 Self-associating assemblies of myelin basic protein . . . . . . . 74

3.2.4 Molecular nature of self-association . . . . . . . . . . . 82

3.2.4.1 $\mathrm{MBP} \mathrm{F} \rightarrow \mathrm{S}$ mutant retains membrane binding capabilities ................... . 84

3.2.4.2 Self-interaction between MBP molecules is promoted by hydrophobicity per se at the site of phenylalanine residues ................... 85

3.2.4.3 FRET measurements reveal less proximity between MBP F $\rightarrow$ S molecules . . . . . . . . . . . . . . 86

3.2.4.4 Measurement of adhesion forces between wild type and $\mathrm{F} \rightarrow \mathrm{S}$ molecules . . . . . . . . . . . . 87

3.2.4.5 Functional consequences of $\mathrm{F} \rightarrow \mathrm{S}$ mutations . . . . . 89

3.2.5 Self-association of MBP promotes macroscopic phase separation 91

4 Discussion

4.1 Model system to study biogenesis of myelin membrane . . . . . . . . 93

4.2 Mechanisms that regulate polarization of oligodendrocytes . . . . . . 94

4.3 Minimal component systems and reconstitution of MBP barrier . . . 98

4.4 Self-assembling diffusion barriers . . . . . . . . . . . . . . . . . . . . . 99

4.5 Protein phase separation in solution . . . . . . . . . . . . . . . . 103

5 Summary and Outlook 105

Bibliography 107

A Appendix

Acknowledgements 



\section{List of Figures}

1.1 Schematic view of major myelin lipids . . . . . . . . . . . . . . . . 4

1.2 Schematic view of myelin proteins . . . . . . . . . . . . . 12

1.3 Schematic view of axo-glia junctions . . . . . . . . . . . . . 15

1.4 Schematic view of polarized myelin along the radial direction . . . . 16

1.5 Examples of lateral diffusion barriers . . . . . . . . . . . . . . 20

3.1 Model system of cultured oligodendrocytes to study myelin membrane biogenesis . . . . . . . . . . . . . . . . . . 44 44

3.2 Localization of cytosolic proteins with decreasing Stoke's radii in oligodendrocytes . . . . . . . . . . . . . . . . . . . . 4 44

3.3 MBP establishes polarity in oligodendrocytes . . . . . . . . . . . . 46

3.4 Localization of endogenous and exogenous proteins with bulky cytoplasmic domains in WT and Shiv cells . . . . . . . . . . . . . 47

3.5 Restoration of polarity in shiverer oligodendrocytes . . . . . . . . . 48

3.6 MBP regulates surface polarity in oligodendrocytes . . . . . . . . . 49

3.7 Fusion of GFP to the cytosolic, but not extracellular side restricts MOG to the processes . . . . . . . . . . . . . 50

3.8 Non-compact myelin proteins lacking cytoplasmic domains redistribute into the myelin membrane sheets . . . . . . . . . . . 51

3.9 Cytoplasmic size limit for entering into the myelin membrane sheets is less than 30 aa . . . . . . . . . . . . . . . . . . 53

3.10 Charge of the cytosolic domain does not influence diffusion into the myelin membrane sheets . . . . . . . . . . . . . . . 54

3.11 FRAP experiments with compact and non-compact myelin proteins 56

3.12 Lipid-to-protein ratios are altered in the shiverer myelin . . . . . . . 57

3.13 Cell surface abundance of proteins in WT vs. Shiv membrane sheets 58 
3.14 In vivo localization of membrane-anchored GFP in myelin . . . . . 59

3.15 Comparison of CNPase distribution within myelin between WT and Shiv mice . . . . . . . . . . . . . . . . . . . . . 60

3.16 Estimation of MBP amounts in primary oligodendrocytes . . . . . . 61

3.17 Reconstituting the sieving function of MBP in a biomimetic assay . 63

3.18 Extrusion of R3-GFP from the areas where GUVs spread onto the SLB in the presence of MBP . . . . . . . . . . . . . . . 64

3.19 Cross-linking of two membrane with antibodies is not sufficient to mimic the function of $\mathrm{MBP} \ldots \ldots \ldots$. . . . . . . . 64

3.20 Extrusion of non-compact myelin proteins from the myelin membrane sheets during oligodendrocyte development . . . . . . . . 66

3.21 In vivo localization of CNPase in myelin during development . . . . 67

3.22 MBP establishes ER-PM domains in PtK2 cells when fused to the C-terminus of an integral membrane protein . . . . . . . . . . . 69

3.23 Reconstitution of MBP barrier function in a cell line . . . . . . . . . 70

3.24 MBP positive ER-PM domains exclude proteins with bulky cytosolic domains . . . . . . . . . . . . . . . . . . . . . . . . . 72

3.25 Cytosolic size limit for entering into MBP positive ER-PM domains is less than 30 aa . . . . . . . . . . . . . . . . . . . . . 73

3.26 MBP positive ER-PM domains exclude glycoproteins . . . . . . . . 74

3.27 Retraction of MBP domains upon redistribution of surface charge . 75

3.28 FRAP experiments to monitor the mobility of MBP domains in PtK2 cells and primary oligodendrocytes . . . . . . . . . 77

3.29 Cross-linking RFP into MBP positive domains and measuring its mobility . . . . . . . . . . . . . . . . . 78

3.30 Photoconversion experiments for monitoring the mobility of MBP domains in PtK2 cells and primary oligodendrocytes . . . . . . . . . 79

3.31 MBP forms higher order structures in vitro as well as in primary oligodendrocytes . . . . . . . . . . . . . . . . . . . . . 81

3.32 Oligomerization requirements for the efficient size barrier formation 82

3.33 MBP $\mathrm{F} \rightarrow \mathrm{S}$ is restricted from entering into the myelin membrane

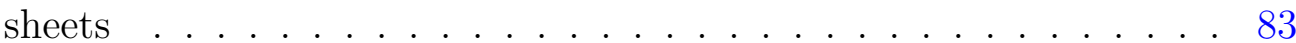


3.34 MBP $\mathrm{F} \rightarrow \mathrm{S}$ retains membrane binding capabilities similar to wild type MBP . . . . . . . . . . . . . . . . . . . 84

3.35 ER-PM domain formation by various MBP mutants in PtK2 cells . 85

3.36 Self-interactions between wild type MBP molecules vs $\mathrm{F} \rightarrow \mathrm{S}$ mutant molecules . . . . . . . . . . . . . . . . . . . 87

3.37 The self-interaction forces between $\mathrm{MBP} \mathrm{F} \rightarrow \mathrm{S}$ mutant are smaller than wild type MBP . . . . . . . . . . . . . . . . . . . . . 88

3.38 Functional consequences of $\mathrm{F} \rightarrow \mathrm{S}$ mutations within MBP sequence . 89

$3.39 \quad \mathrm{~F} \rightarrow \mathrm{S}$ mutant fails to rescue polarity loss in shiverer cells . . . . . . 90

3.40 Mesoscopic phase separation of MBP in solution . . . . . . . . . . . 91

4.1 A diffusion barrier generates polarized myelin membrane sheets . . . 95 



\section{List of Tables}

2.1 Antibodies used in this study . . . . . . . . . . . . . . . 23

2.2 Buffers and solutions used in the study . . . . . . . . . . . 25

2.3 Enzymes used in this study . . . . . . . . . . . . . . . 26

2.4 Commercial kits used in this study . . . . . . . . . . . . 26

2.5 Cell lines and bacterial strains employed in the study . . . . . . . . 27

2.6 List of softwares used in the study . . . . . . . . . . . . . . . . . . . 41

A.1 List of constructs . . . . . . . . . . . . . . . . . . 131

A.2 List of primers . . . . . . . . . . . . 136 



\section{Abbreviations}

\begin{tabular}{ll} 
A & Alanine \\
aa & Amino acids \\
ab & Antibody \\
AIS & Axon initiation segment \\
AnkG & Ankyrin G \\
APS & Ammonium persulfate \\
BSA & Bovine serum albumin \\
BCA & Bicinchoninic acid \\
CAM & Cell adhesion molecule \\
Caspr & Contactin associated protein \\
Cit & Citrulline \\
CHAPS & 3 -[(3-cholamidopropyl)dimethylammonio]-1-propanesulfonate \\
CNPase & $2^{\prime}, 3^{\prime}$-cyclic-nucleotide $3^{\prime}$-phosphodiesterase \\
CNS & Central nervous system \\
ConA & Concanavalin A \\
D & Aspartate \\
DFDNB & 1,5-Difluoro-2,4-dinitrobenzene \\
DIV & Days in vitro \\
DMA & Dimethyl adipimidate.2HCl \\
DMEM & Dulbecco's modified eagle's medium \\
DMP & Dimethyl pimelimidate.2HCl \\
DMS & Dimethyl suberimidate.2HCl \\
DMSO & Dimethyl sulfoxide \\
DNA & Deoxyribonucleic acid \\
DSG & Disuccinimidyl glutarate \\
& \\
\hline
\end{tabular}


DSS Disuccinimidyl suberate

DTT Dithiothritol

E Glutamate

E.coli Escherichia coli

EDTA Ethylenediaminetetraacetic acid

EM Electron microscopy

ER Endoplasmic reticulum

F Phenylalanine

FCS Fetal calf serum

FRAP Fluorescence recovery after photobleaching

FDAP Fluorescence decay after photoconversion

FRET Fluorescence resonance energy transfer

FLIP Fluorescence loss in photobleaching

G Glycine

GAP GTPase activating protein

GalC Galactosylceramide

GDP Guanosine diphosphate

GFP Green fluorescent protein

GLC Gas liquid chromatography

GTP Guanosine triphosphate

GUV Giant unilamellar vesicle

HBSS Hank's balanced salt solution

HEPES 4-(2-Hydroxylethyl)piperazine-1-ethanesulfonic acid

hrs Hours

HPLC High pressure liquid chromatography

HRP Horseradish peroxidase

HS Horse serum

$\mathrm{Hz} \quad$ Hertz

I Isoleucine

Ig Immunoglobulin

IPTG Isopropyl $\beta$-D-thiogalactopyranoside

IUPs Intrinsically unstructured proteins

K Lysine

$\mathrm{kDa} \quad$ Kilodalton 
LUV Large unilamellar vesicle

$\mathrm{PH} \quad$ Plekstrin homology

PLP Proteolipid protein

PMD Pelizaeus-Merzbacher disease

MAG Myelin-associated glycoprotein

MAL Myelin and lymphocyte

MARKS Myristoylated alanine-rich C kinase substrate

MBP Myelin basic protein

min Minute(s)

mld myelin deficient

MOBP Myelin oligodendrocyte basic protein

MOG Myelin oligodendrocyte protein

Necl Nectin-like

Nfas155 Neurofascin 155

Nfas186 Neurofascin 186

OPCs Oligodendrocyte precursor cells

PAGE Polyacrylamide gel electrophoresis

PBS Phosphate buffered saline

PC Phosphatidylcholine

PCR Polymerase chain reaction

PE Phosphatidylethanolamine

PFA Paraformaldehyde

PIP2 Phosphatidyl-4,5-bisphosphate

PLL Poly-L-lysine

PM Plasma membrane

PNS Peripheral nervous system

pOL Primary oligodendrocyte

PS Phosphatidylserine

R Arginine

RFP Red fluorescent protein

ROI Region of interest

rpm Rotations per minute

RT Room Temperature

S Serine 
SD Standard deviation

SDS Sodium dodecyl sulphate

sec Second (s)

SEM Standard error of mean

Shiv Shiverer

SLBs Supported lipid bilayers

SM Sphingomyelin

SUV Small unilamellar vesicle

TAG Transient axonal glycoprotein-1

TCEP Tris-(2-Carboxyethyl)phosphine, Hydrochloride

TEMED N,N, $\mathrm{N}^{\prime}, \mathrm{N}^{\prime}$-Tetramethylethane-1,2-diamine

TGN Trans-Golgi network

UTR Untranslated region

VLFA Very long chain fatty acids

WGA Wheat germ agglutinin

WT Wild type

Y Tyrosine

YFP Yellow fluorescent protein 


\section{Abstract}

Myelin is a membrane of vital importance. In the vertebrate nervous system, it promotes rapid conduction of nerve impulses via insulating the axons. To act as an insulator myelin needs to assemble into a stable, lipid-rich structure. While the biochemical composition of myelin is well described, mechanisms regulating this unique molecular composition are poorly understood. In this study, we show that oligodendrocytes employ a molecular sieve to filter out membrane proteins from compact myelin domain. Myelin basic protein (MBP) forms a size barrier and controls the entry of proteins into the compact myelin based on the bulkiness of their cytoplasmic domains. In fact, only proteins with cytosolic domain of less than 30 amino acids cross the permeability barrier. Mechanistically, we show that after binding to the inner leaflet of the myelin bilayer, MBP self-associates and phase separates. Self-assembly requires hydrophobic interactions between the phenylalanine residues. Replacing phenylalanine residues with serines abolished the self-association between the MBP molecules without perturbing membrane binding capabilities. We further show that during development, molecular self-assembly of the MBP molecules is required for the extrusion of bulky proteins from the compacted myelin membrane sheets. This system might have evolved in oligodendrocytes in order to generate an anisotropic membrane organization that facilitates the assembly of highly insulating lipid-rich membranes. 

"Nothing is more practical than a good theory."

Kurt Lewin

\section{Introduction}

\subsection{Myelin}

Myelin is a mutilayered stack of tightly packed membranes that are wrapped around the axons in the central nervous system (CNS) and the peripheral nervous system (PNS). While oligodendrocytes synthesize this membrane in the CNS, Schwann cells do so in the PNS. Both these cell types expand enormous amount of membranes that are closely apposed on the cytosolic as well as extracellular side. These wrappings effectively increase the total membrane resistance, decrease membrane capacitance and therefore prevent leakage of current along the myelinated segments. The main function of myelin involves wrapping and insulation of axons which in turn leads to saltatory propagation of nerve impulses.

\subsection{Oligodendrocyte differentiation}

Oligodendrocyte precursor cells (OPCs) originate from proliferating neuroepithelial cells of the ventricular and subventricular zone. Basic helix-loop-helix transcription factor Olig2 plays an important role in defining the oligodendrocyte lineage cells. Upon activation, Olig2 triggers the expression of a number of transcription factors like Olig1, Asc1, Nkx2.2, Sox10, YY1, MRF and Zfp19 (Wegner, 2008; Emery, 2010). In the Olig2-null mice, cells of oligodendrocyte lineage are completely missing (Zhou et al., 2001; Lu et al., 2002). OPCs can be identified by the presence of markers like A2B5 antigen and chondroitin sulfate proteoglycan, NG2 (Raff, 1989). Proliferation of OPCs is under the control of growth factors like platelet derived growth factor (PDGF) and fibroblast growth factor (FGF) (Noble et al., 1988; McKinnon et al., 1991). During development, precursor cells migrate under the influence of extra- 
cellular matrix and populate the entire brain. After migration, progenitors settle along the future white matter fiber tracts. Further, the cells become less motile, lose mitogenic response to PDGF and develop into immature oligodendrocytes. At this stage, cells also lose A2B5 expression and instead begin to express galactosylceramide (GalC) (Bansal and Pfeiffer, 1997; Pfeiffer et al., 1993). The final stage of oligdendrocyte development involves maturation into myelinating oligodendrocytes. In this stage, cells express myelin membrane components like MBP and PLP, thereby triggering myelin assembly.

\subsection{Myelin architecture}

Myelin is made up of multilayered stacks of uniformly thick membrane. It shows a characteristic periodic structure with alternating electron dense and light layers visible under an electron microscope. The electron dense structures correspond to the major dense line, which is formed due to the close association of myelin membranes on their cytoplasmic side. On the other hand, the intraperiod line is the electron light structure where myelin membranes are apposed closely on their extracellular surface. As a result, myelin membrane sheaths are compacted on the cytosolic as well as extracellular side. Myelin is wrapped around the axons of a certain caliber $(>1 \mu \mathrm{m})$. While an oligodendrocyte can wrap up to 40 different axons at a time, a Schwann cell establishes 1:1 contact with an axonal segment. One myelin segment can be 150-200 $\mu \mathrm{m}$ in length and is termed as an internode. The internodal distances within the same axon are relatively constant and seem to be inherent for individual axons. The two internodes are separated by the unmyelinated region that corresponds to the nodes of Ranvier (discussed in detail later). An internodal myelin is polarized into two major domains, the compact and the non-compact myelin. Each domain contains specific set of proteins and thus has unique molecular composition. Compact myelin forms the bulk of myelin and is mainly composed of lipids. On the other hand, the majority of the cytoplasm and vesicular trafficking is restricted to the non-compact myelin areas that are mainly present as channels surrounding the compact myelin in the CNS. In PNS, the non-compact areas also extend within the compact myelin and correspond to the so called Schmidt-Lanterman incisures. 


\subsection{Myelin lipid composition}

Lipids constitute up to $80 \%$ of the dry weight of myelin (Norton and Autilio, 1965). It is because of this high lipid-to-protein ratios ( 4 in comparison to $0.25-1$ for the plasma membrane), myelin can be obtained in a relatively pure form. The purification method involves homogenization of the brain tissue in an isotonic sucrose solution to detach myelin from the axons and thereby generate large vesicles. The high lipid content makes these myelin membrane vesicles lighter than the intracellular organelles (nuclei and mitochondria). As a result, myelin membrane can be recovered from top of the lighter fractions in a density gradient centrifugation (Norton and Poduslo, 1973; Larocca and Norton, 2007).

Lipid analysis of biochemically purified myelin has shown that cholesterol constitutes more than $40 \%$ of the total lipid content. Cholesterol is an amphiphilic molecule with polar hydroxyl group and non-polar cyclic four-ring structure together with isooctyl side chain (Figure 1.1). Cholesterol interacts with the polar head groups of other lipids through the hydroxyl group. At the same time, via its rigid planar structure, cholesterol intercalates between saturated hydrocarbon chains of adjacent lipids. These properties allow cholesterol to control various parameters like membrane thickness and membrane fluidity. Insights into the role of cholesterol in myelin membrane biogenesis came from the study of squalene synthase conditional knock-out mice. As this enzyme operates at the first step committed towards sterol biosynthesis, mice lacking squalene synthase are not able to synthesize sterols. These mice show severely perturbed myelin synthesis, early in development which seems to be compensated with aging by cholesterol uptake from the neighboring cells (Saher et al., 2005, 2011). The exact mechanism of this horizontal transfer remains to be elucidated.

Another major lipid class in myelin includes galactolipids, with galatosylceramide and sulfatide being the two major ones (30\% of the total lipids). These lipids are essentially localized to the extracellular leaflet of the bilayer. The role of galactolipids in myelin membrane assembly was investigated via generation of knock-out mice 


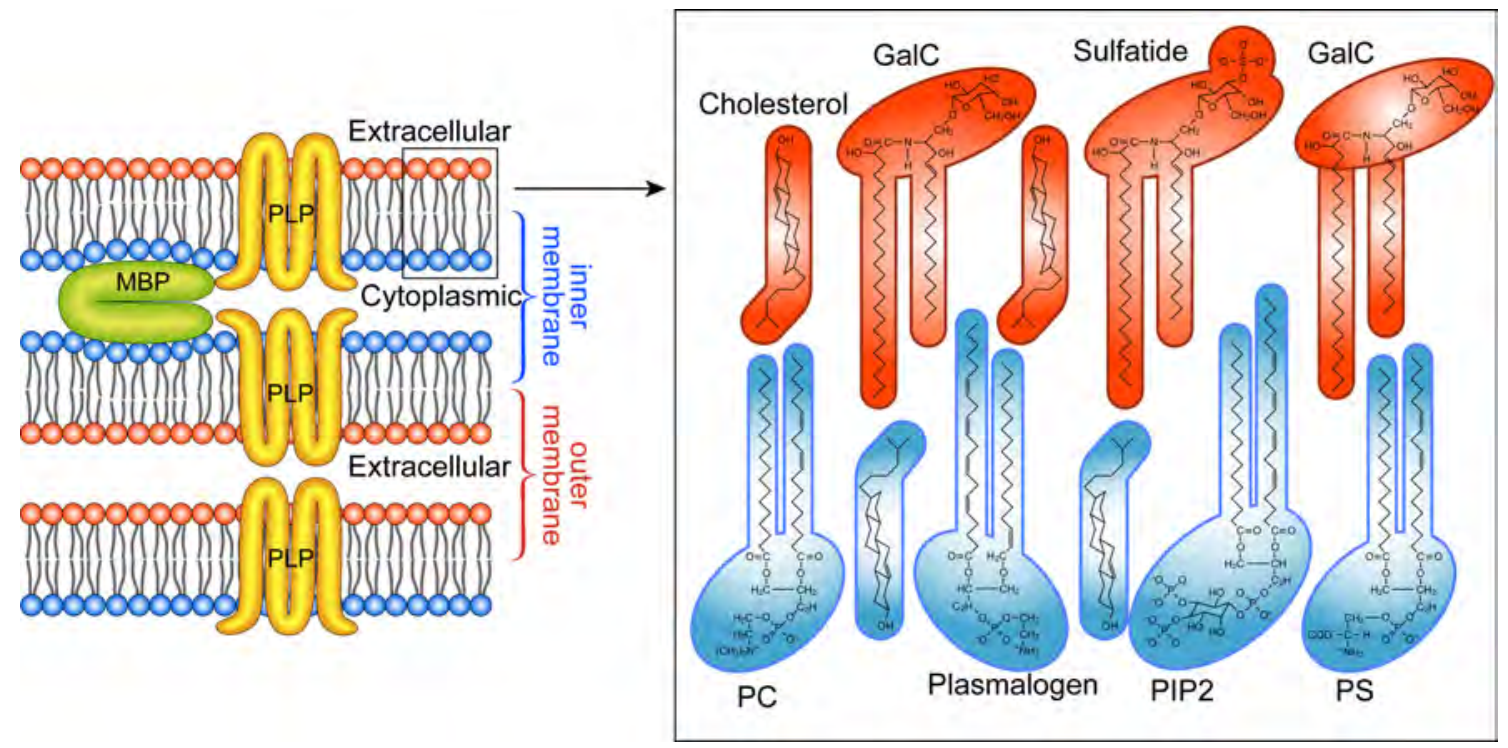

Figure 1.1: Schematic view of major myelin lipids. Compact myelin is formed by the apposition of the external surfaces and internal surfaces of the myelin bilayer that constitute the intraperiod line and the major dense line, respectively. Proteolipid protein (PLP) and myelin basic protein (MBP) are the two major proteins in myelin. The myelin bilayer has an asymmetric lipid composition. Some of the major lipid classes are shown on right. Lipids like galactosylceramide (GalC) and sulfatide are exclusively found in the outer leaflet. On the other hand negatively charged lipids, phosphatidylserine (PS) and phosphatidylinositol-4,5-bisphosphate (PIP2) are localized to the inner leaflet together with plasmalogens. Cholesterol and phosphatidylcholine (PC) are distributed in both the leaflets. Note the intercalation of cholesterol molecules between the fatty acid chains. Figure adapted from (Aggarwal et al., 2011).

lacking key enzymes in the biosynthesis pathway of these lipids. CST knock-out mice lack cerebroside sulfotransferase and cannot synthesize the lipid sulfatide. On the other hand, CGT knock-out mice do not produce sulfatide as well as galactosylceramide due to the deficiency of UDP-galactose:ceramide galactosyl transferase. Both of these mice form structurally abnormal myelin with disrupted nodes and paranodes (Coetzee et al., 1996; Marcus et al., 2006; Hoshi et al., 2007).

A unique feature of cerebrosides and sulfatides within myelin is their enrichment in very long chain fatty acids (VLFA), with chain lengths ranging between 22-24 carbons. Ceramide synthase 2 (CerS2) specifically catalyzes addition of VLFA to 
the sphingosine base. Expression of this enzyme transiently increases during active myelination (Becker et al., 2008). Mice deficient in CerS2 show progressive loss of CNS and PNS myelin together with decreased levels of MBP (Imgrund et al., 2009). Further, time course analysis of these mice revealed that the initial myelin formation per se is normal, but myelin maintenance is impaired (Ben-David et al., 2011).

Finally, plasmalogens account for $20 \%$ of the myelin phospholipid mass. These are ether phosphoethanolamines containing a vinyl ether linkage at the $s n-1$ position and an ester linkage at the $s n-2$ position. Plasmalogens increase membrane fluidity by lowering the transition temperature for phospholipids from lamellar to a liquid crystalline phase. Deficiency of dihydroxyacetone phosphate acyltransferase (DAPAT), the key enzyme for plasmalogen synthesis results in reduced myelin synthesis in optic nerve and cerebellum (Teigler et al., 2009).

\subsection{Myelin protein composition}

Proteins constitute about $20-30 \%$ of the dry weight of myelin. Out of this, proteolipid protein (PLP) and myelin basic protein (MBP) constitute $80 \%$ of the total protein content. Other abundant proteins in the CNS myelin include myelin-associated glycoprotein (MAG), myelin oligodendrocyte glycoprotein (MOG), myelin oligodendrocyte basic protein (MOBP), 2' $3^{\prime}$-cyclic-nucleotide $3^{\prime}$-phosphodiesterase (CNPase), myelin and lymphocyte tetraspan protein (MAL), claudin 11, neurofascin-155 and opalin/Tmem10 (Figure 1.2). Some of these proteins are important for both, the structure and function of myelin, whereas the function of others are unknown, as will be discussed below.

\subsubsection{Proteolipid protein}

PLP is the most abundant protein of CNS myelin and accounts for $50 \%$ of the total myelin proteins. It is a four-membrane spanning protein with intracellular $\mathrm{N}$ - and C-terminus. It has a molecular weight of $20 \mathrm{kDa}$. Due to its extremely hydrophobic nature, PLP exists in close association with lipids (Weimbs and Stoffel, 1992) and 
can therefore be extracted from myelin only with organic solvents (Folch and Lees, 1951). It is strongly conserved during evolution (Möbius et al., 2008). Further, it is considered as the structural protein with an important role in stabilizing the intraperiod line, mainly via the extracellular domain (Boison and Stoffel, 1994; Boison et al., 1995). PLP has an alternatively spliced isoform, DM20 which lacks 35 amino acids in the intracellular domain, but has similar physical properties (Macklin et al., 1987; Nave et al., 1987). In comparison to PLP, DM20 appears very early during oligodendrocyte differentiation in pre-myelinating oligodendrocytes.

PLP is synthesized on membrane-bound ribosomes associated with the ER (Colman et al., 1982) and is transported to the developing myelin sheath via vesicular trafficking pathway. Briefly, after synthesis in the ER, the protein is transported to the Golgi where it associates with cholesterol and galactosylceramide (Simons et al., 2000). Finally, vesicles bud off from the Golgi and are delivered to the growing myelin membrane sheath. Delivery of PLP to the surface of myelin membrane is regulated by neuronal signals that seem to reduce its endocytosis rate and instead promote delivery to the plasma membrane from late endosomes/lysosomes (Trajkovic et al., 2006).

Gene duplication, mutation or deletions in the PLP gene lead to dysmyelinating diseases like Pelizaeus-Merzbacher disease (PMD) and X-linked spastic paraplegia (Yool et al., 2000). In primary oligodendrocyte cultures, overexpressed PLP associates with cholesterol and accumulates in the late endosome/lysosomes (Simons et al., 2002). In the mouse model of PMD, intracellular accumulation of PLP could be reduced via feeding mice with cholesterol enriched diet (Saher et al., 2012). Point mutations in PLP have been shown to result in ER retention due to abnormal protein cross-linking that elicits enfolded protein response (Swanton et al., 2005; Dhaunchak and Nave, 2007). Unlike mutations and duplications in PLP gene, mutant mice lacking PLP expression form relatively normal myelin during early development. However, with age these animals exhibit axonal degeneration indicating that myelin deficient in PLP is not able to efficiently support the axons (Griffiths et al., 1998). 


\subsubsection{Myelin basic protein (MBP)}

Another abundant protein in myelin is myelin basic protein (MBP). It comprises $30 \%$ of the total myelin proteins and $10 \%$ of the dry weight of myelin. MBP is a peripheral membrane protein. It is a highly positively charged protein with a pI of 10 and can associate with a number of ligands. The most important ones are the negatively charged lipids present in the inner myelin leaflet, namely phosphatidylserine (PS) and phosphatidylinositol-4,5-bisphosphate (PIP2) (Boggs and Moscarello, 1978). Electrostatic interactions with these lipids are thought to mediate the interaction of MBP with the plasma membrane. Apart from lipids, MBP has also been shown to bind actin, calmodulin, tubulin and clathrin in the in vitro experiments (Boggs, 2006). Due to interaction with multiple ligands, MBP is also termed as a multifunctional protein.

\section{MBP structure}

MBP belongs to the family of intrinsically unstructured proteins (IUPs) (Hill et al., $2002,2003)$. In this family, proteins are natively unfolded in the absence of a binding partner (Tompa, 2002; Fink, 2005). The main reason for the lack of structure is the abundance of charged amino acids like glutamate, arginine, lysine and serine that increase intramolecular electrostatic repulsions. In addition, the overall hydrophobicity is low compared to globular proteins (Romero et al., 2001; Dunker et al., 2002). As a result, the chances of hydrophobic collapse are minimum and proteins remain unstructured in solution.

Even though IUPs are unfolded in solution, ligand binding induces conformational changes within IUPs. MBP acquires both $\alpha$-helices and $\beta$-sheets upon binding to the charged lipids (Keniry and Smith, 1979; Polverini et al., 1999). In the folded state, IUPs are involved in a wide variety of cellular functions like cell cycle control, transcriptional and translational regulation.

For some IUPs like MARKS (myristoylated alanine-rich C kinase substrate), K-Ras and HIV-1 Gag, the unstructured basic stretch is concentrated in one region of the 
protein (Murray et al., 1997). In case of MBP, the scenario is little different as the basic residues are distributed throughout the length of the protein. This uniform distribution has been considered to be important for simultaneous binding of the protein to the opposing membranes. Indeed both $\mathrm{N}$ - and C-terminus of MBP have been shown to independently associate with lipids (Boggs et al., 1999).

\section{MBP-lipid interactions}

The association of MBP with the membrane has been mainly considered to be electrostatic in nature. Upon addition to lipid vesicles containing negatively charged and neutral lipids, MBP displays preferential binding for the negatively charged species (Boggs et al., 1977). In vitro, MBP aggregates lipid vesicles containing negatively charged lipids causing multilayer formation (Wood and Moscarello, 1989). When expressed in oligodendroglial precursor cells, MBP binds to the plasma membrane in a PIP2 dependent manner (Nawaz et al., 2009). It also sequesters the lipid, PIP2 on the surface of large unilamellar vesicles (LUVs) (Musse et al., 2008). These attractive electrostatic interactions between lipids and MBP closely depend on the ratios between the lipid and protein charges (Hu et al., 2004; Min et al., 2009).

Even though the major interaction between MBP and lipids is electrostatic, hydrophobic interactions may also play an important role. In the lipid aggregation experiments, high $\mathrm{K}^{+}$concentration $(>150 \mathrm{mM}$ ) causes dissociation of MBP from PC/PS vesicles, but not from vesicles with complex, inner myelin leaflet composition (Jo and Boggs, 1995). Based on above observation, the authors speculate that the protein can interact with the lipid head groups either via hydrogen bonding or through hydrophobic interactions. Through hydrophobic association, the side chains of the non-polar residues can bury into the bilayer. In fact, it has been demonstrated that MBP affects the motion of fatty acid chains, buried deep within the bilayer (Marsh et al., 2002). Penetration of certain regions of the protein in the bilayer possibly affects membrane order as well as domain organization (Harauz et al., 2009). For example, MBP plays a role in the clustering of outer leaflet lipid, GalC (Fitzner et al., 2006). Further, dramatic rearrangements and morphological changes have been observed in lipid monolayers upon MBP adsorption (Hu and Is- 
raelachvili, 2008). These experiments clearly indicate a role of MBP in generating lateral heterogeneity.

\section{Charge isomers of MBP}

The net charge of MBP can be up to +20 at the physiological $\mathrm{pH}$. However, the extent of post-translational modifications can modify the overall charge of the protein. For example, phosphorylation of serine and threonine residues, deimination of the arginine (arg) residues to citrulline (cit), and deamidation reduce the overall positive charge (Harauz et al., 2004). C1 and C8 represent two extremes amongst various charge isomers. While $\mathrm{C} 1$ is least modified and has maximum charge, $\mathrm{C} 8$ is extensively deiminated. Each deimination reduces the net charge of protein by one. 6-8 arg residues are deiminated to cit in the $\mathrm{C} 8$ isoform. Because of reduced charge, C8 is less efficient than C1 in adhering apposed membranes (Boggs et al., 1997; Musse et al., 2006).

\section{MBP isoforms}

Apart from charge isomers, MBP has different size isoforms with molecular weight ranging between 14 and $21.5 \mathrm{kDa}$. These isoforms arise from the differential splicing of a single mRNA transcript. $18 \mathrm{kDa} \mathrm{MBP}$ is the major isoform in human and bovine CNS. On the other hand, $14 \mathrm{kDa}$ is the most abundant isoform in mice and rats. Both, 14 and $18 \mathrm{kDa} \mathrm{MBP}$ isoforms contain exon I, III, IV, VI and VII. Exon II is present in the $21.5 \mathrm{kDa}$ MBP. This isoform appears early in development, while the other isoforms are expressed later during myelination (Kamholz et al., 1988). Unlike 14 and $18 \mathrm{kDa}$ isoforms that are mainly associated with the membrane, 21.5 $\mathrm{kDa}$ isoform localizes to the nucleus (Pedraza, 1997). Sequence analysis of exon II revealed the presence of two non-traditional PY-nuclear localization signals (Smith et al., 2012). The functional role of subcellular trafficking to the nucleus remains to be elucidated.

\section{Shiverer mice}

The importance of MBP in myelination is highlighted by the naturally occurring autosomal recessive shiverer mutant mice. These mice lack exons $2-7$ within the 
MBP gene and are therefore unable to synthesize all the isoforms of MBP. At the age of 2 weeks, corresponding to the peak phase of myelination, animals begin to develop shivering phenotype in their movement. Later on, the mutants develop tonic convulsions and die prematurely, between 8-11 weeks after birth (Chernoff, 1981). Ultra-structurally, major dense line is missing in these mice as visualized by electron microscopy (Privat et al., 1979). Furthermore, myelin is also thinly packed and the axo-glia junctions are diffusely distributed (Rosenbluth, 1980).

Extensive studies have been carried out in the past to demonstrate the direct involvement of MBP in myelin compaction. Shiverer phenotype was rescued by the introduction of full-length MBP gene coding for all the isoforms of MBP (Readhead et al., 1987). Apart from full-length MBP, shiverer phenotype in mice could also be rescued via re-expression of only $14 \mathrm{kDa}$ isoform of MBP under MBP promoter (Kimura et al., 1989). Further, introduction of MBP anti-sense cDNA in the genome of wild type mice evoked shivering response (Katsuki et al., 1988). Yet another naturally occurring MBP mutant is known as myelin deficient (mld). In these mice, inversion of exon 2 in the duplicated MBP gene leads to the formation of anti-sense RNA, which inhibits MBP transcription (Akowitz et al., 1987; Okano et al., 1987). As a result, the phenotype is similar to the shiverer mice. MBP, thus seems to be a very important player in the architectural design of myelin.

\section{Regulation of MBP expression}

Given the role of MBP within myelin, spatio-temporal regulation of its expression is important to finely regulate myelin biogenesis. Nature has achieved this via localized MBP synthesis close to its incorporation sites. MBP mRNA, once formed in the cell body is kept silent and is transported towards the cellular processes. The first hints for localized mRNA transport came from the biochemical fractionation studies where isolated crude myelin fractions were found to be enriched in MBP mRNA (Colman et al., 1982). Subsequently in situ hybridization experiments confirmed RNA enrichment near the sites of myelin membrane (Kristensson et al., 1986; Verity and Campagnoni, 1988). 
Localization of MBP mRNA to the sites of myelin membrane assembly occurs in a series of steps. First, the cellular address of the transcript is defined by a 21 nucleotide cis-acting element, termed "RNA trafficking signal" (RTS), present in the $3^{\prime}$ untranslated region (UTR). This sequence was shown to be sufficient for the localization of an exogenous RNA into the myelin compartment (Ainger et al., 1997; Munro et al., 2006). In the next step, ribonucleoproteins (RNA binding proteins) bind to the RTS to form supramolecular discrete structures termed "RNA granules" (Ainger et al., 1993). HnRNP A2, hnRNP CBF-A and hnRNP K are the major ribonucleoproteins, which directly associate with the RTS and block the translation of MBP mRNA (Hoek et al., 1998; Raju et al., 2008; Laursen et al., 2011). Other proteins like hnRNP E1, hnRNP F and tumor overexpressed gene (TOG) interact with hnRNP A2 and thus indirectly associate with the RNA granules. While hnRNP E1 and hnRNP F act in the same pathway as hnRNP A2 and represses translation, TOG seem to be important for the efficient translation of mRNA (Kosturko et al., 2006; Francone et al., 2007; White et al., 2011). Apart from the RNA and associated ribonucleoproteins, RNA granules also contain various ribosomal subunits, translation factors and tRNAs (Barbarese et al., 1995). Following RNA granule assembly in the perikaryon, RNA complexes travel in the anterograde direction along microtubule tracks, using kinesin motor proteins. Disruption of microtubules with drugs like nocodazole or knockdown of kinesin blocks the granule transport (Carson et al., 1997). The final and essential step necessary for appropriate myelination is the release of translation repression near the assembly sites, either by pre-localized proteins or in response to cellular signaling. In vitro, association of neural adhesion molecule L1 with oligodendrocytes activates Fyn kinase, which phosphorylates and thereby inactivates hnRNP A2 and hnRNP F (Umemori et al., 1999; White et al., 2008). As a result, transcription repression is relieved and MBP protein synthesis is triggered. The transport of mRNAs from cell body to the distal parts in a polarized cell adds another layer of regulation in the functional domain assembly. 


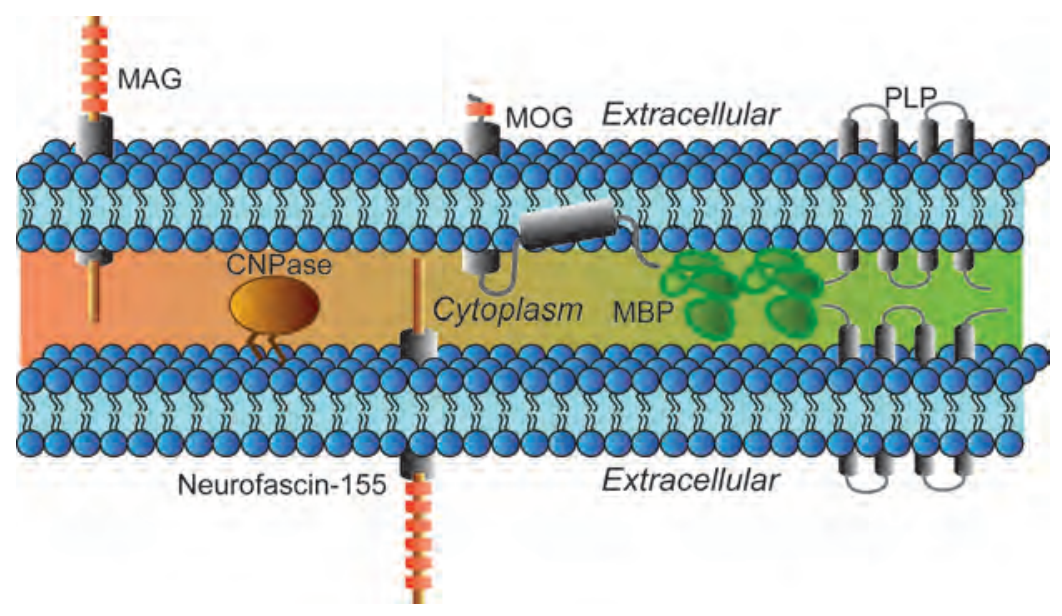

Figure 1.2: Schematic view of myelin proteins. Myelin basic protein (MBP) and proteolipid protein (PLP) are the two major compact myelin proteins. The non-compact myelin proteins include membrane anchored, CNPase and single transmembrane spanning myelin-associated glycoprotein (MAG) and $155 \mathrm{kDa}$ isoform of neurofascin (Neurofascin-155).

\subsubsection{Other myelin proteins}

\section{Claudin-11}

Apart from PLP and MBP, claudin 11 is also present within compacted myelin (Bronstein et al., 1996). It belongs to the tetraspanin protein family and constitutes $7 \%$ of the total myelin proteins (Bronstein et al., 1997). Like PLP, both N- and Cterminus for claudin-11 are intracellular. The protein forms the radial component within compacted myelin, which isolates the myelin sheaths from the extracellular space. Claudin-11 knock-out mice lack the radial component (Gow et al., 1999) and exhibit decreased nerve conduction velocities (Devaux and Gow, 2008).

\section{MAG}

Myelin-associated glycoprotein (MAG), a single transmembrane protein, is another component of myelin. It is mainly present in the periaxonal space, region between the axon and the innermost layer of myelin (Trapp and Quarles, 1982). The protein belongs to immunoglobulin (Ig) superfamily with five extracellular Ig-like domains. Within myelin, MAG exists in two isoforms: large L-MAG and small S-MAG, which differ in the length of cytosolic domain. L-MAG has 90 aa on the cytoplasmic side 
and is expressed early during myelination. On the other hand, S-MAG has only 46 aa and appears in the later stages of development. Mice deficient in MAG assemble normal myelin (Li et al., 1994), however axonal caliber is affected, which leads to progressive axonal degeneration (Yin et al., 1998).

\section{MOG}

Myelin oligodendrocyte glycoprotein (MOG) is another single transmembrane protein with a molecular mass of $28 \mathrm{kDa}$. It has a single Ig-like domain on the extracellular side (Pham-Dinh et al., 1993). MOG is present mainly in the outermost layer of myelin. Although exact function of MOG in myelin is not clear, it has been described as a putative auto-antigen in demyelinating diseases such as multiple sclerosis (Bernard et al., 1997).

\section{CNPase}

2',3'-cyclic-nucleotide $3^{\prime}$-phosphodiesterase or CNPase localizes to certain regions within non-compact myelin including the lateral loops, inner and outer tongue (Vogel and Thompson, 1988). The protein has a C-terminal farnesyl lipid anchor for membrane association. It is one of the proteins that are expressed early during myelination. Exact function of CNPase in myelin is not clear. CNPase mutant mice develop axonal degeneration despite normal myelination (Lappe-Siefke et al., 2003).

\section{MAL}

Myelin and lymphocyte protein (MAL) is a small hydrophobic, four membrane spanning protein. It has been shown to localize to the compact myelin. Transgenic mice with increased MAL expression have abnormal axo-glia junctions (Frank et al., 2000). In MAL knock-out mice, paranodal loops detach and face away from the axons (Schaeren-Wiemers et al., 2004). Similar phenotype has been observed for the sulfatide deficient, CGT knock-out mice. The exact relevance of this overlapping function has not yet been described.

Apart from above mentioned proteins, several other proteins have been found to be enriched in myelin. Proteins belonging to tetraspanin family (CD9, CD81, CD82 and 
tetraspanin 2) and septin family are also present in myelin. Furthermore, proteomic analysis of purified myelin membrane fractions has revealed the presence of a number of other proteins which are present in very low proportions (Jahn et al., 2009). Insights into the functional and structural role of these new proteins would increase our understanding about myelin assembly process.

\subsection{Myelin Domains}

Initiation of polarization in a cell requires integrated action of intrinsic signals and extracellular cues. Extrinsic factors can position and assemble intracellular complexes, which define a specific domain. This pathway is well known in case of Schwann cells. As these cells contact the axon, neuregulin1/ErbB receptor signaling is activated. ErbB2 receptors have been shown to be important for myelination (Garratt et al., 2000). Furthermore, the extent of NRGIII expression controls thickness of myelin sheaths (Michailov et al., 2004). In a striking contrast to the PNS, the master regulator of CNS myelination is not known. In fact, initial events of polarization are known to be intrinsic to the oligodendrocytes and occur without axonal contact. However, further polarization into distinct membrane domains does require axonal contact. In the following section, various domains within myelin membrane are described in terms of their structural organization and functional relevance.

\section{Nodes of Ranvier}

Along the myelinated axon, non-insulated regions constitute the nodes of Ranvier (Figure 1.3). Due to the lack of myelin, these regions are directly exposed to the extracellular space. They are about $1 \mu \mathrm{m}$ in size and have high density of voltage gated $\mathrm{Na}^{+}$channels. In addition, several other channels, transmembrane and cytoskeletal proteins localize to the nodes. These include NrCAM and neurofascin-186 (Nfas186), the cell adhesion molecules (CAMs) of immunoglobulin (Ig) superfamily; ankyrin $\mathrm{G}$ (AnkG), the cytoskeletal adaptor protein (Bennett and Lambert, 2000) and $\beta \mathrm{IV}$-spectrin, the actin binding protein. The ankyrin family is known to play a general role in membrane organization and polarity (Mohler et al., 2002). While nodes are the sites where action potentials regenerate, the segment where the ac- 


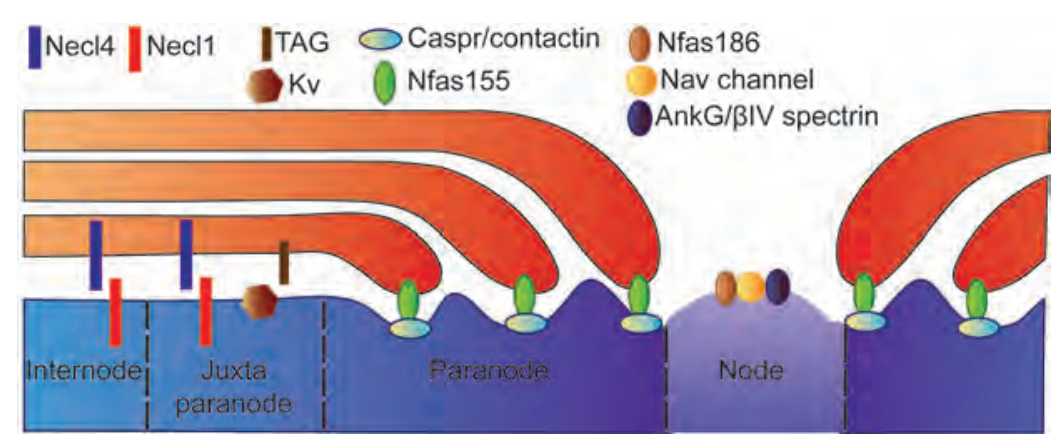

Figure 1.3: Schematic view of axo-glia junctions. Myelinated segments correspond to internodes. Nodes of Ranvier constitute the unmyelinated regions along the myelinated axonal tracks and are enriched in sodium channels (Nav channel). Additionally neurofascin-186 (Nfas186), ankyrin $\mathrm{G}$ (AnkG) and $\beta$ IV-spectrin are also present at the nodes and associate with Nav chanels. Compacted myelin opens around the nodes to form the paranodes. Neurofascin-155 (Nfas155) from the glial side forms complex with axonal contactin-associated protein (caspr) and contactin at the paranodes. Away from the node and next to paranodes are the juxtaparanodes, which are enriched in the potassium channels (Kv). Modified from (Sherman and Brophy, 2005).

tions potentials originate is known as the axon initiation segment (AIS). As the name suggest, AIS is present immediately after the end of cell body and precedes the axon. The molecular architecture/composition is very similar between AIS and a node.

\section{Paranodes}

Around the nodes of Ranvier, compact myelin opens up on both sides to form the cytoplasm filled paranodal loops. These loops helically wind around an axon. At the ultra-structural level, paranodes are connected to the axon by a series of transverse bands, which are reminiscent of the invertebrate septate junctions (Rosenbluth et al., 1995). Paranodal junctions contains a complex of two cell recognition molecules: contactin-associated protein (caspr) and contactin on the axonal side. From the glial side, neurofascin-155 (Nfas155) serves as the ligand for caspr-contactin complex (Charles et al., 2002; Gollan et al., 2003). In caspr-2 knock-out mice, septa disappear and space between the axon and paranodal loops widens. As a result, these mice show decreased nerve conduction velocities and several other neurological symptoms in both, central and peripheral nervous system. A very similar phenotype 
is observed for CGT and CST-null mice (Coetzee et al., 1996; Marcus et al., 2006; Hoshi et al., 2007). These two mutants also provided the first hints that probably axons cannot intrinsically polarize the distribution of molecules to the axo-glia junction, but indeed require intimate contact with the glial membrane. The role of axo-glia junctions in polarity establishment will be discussed in later sections.

\section{Juxtaparanodes}

A short zone that immediately follows the innermost paranodal junction constitutes the juxtaparanodes. On the glial side, juxtaparanodes contain TAG-1 (Transient axonal glycoprotein-1), while shaker based potassium channels are present on the axonal side (Poliak and Peles, 2003). Furthermore, juxtaparanodes together with internodes also harbor nectin-like molecule 1 and 4 (Necl1 and Necl4 respectively). While Necl1 is present on the axonal side, Necl4 is found on the glial side. Necls belong to immunoglobulin like family of adhesion molecules (Takai et al., 2003). Via heterophilic adhesion, they maintain axo-glia adhesion at the juxtaparanodes and internodes (Quarles, 2007; Spiegel et al., 2007).

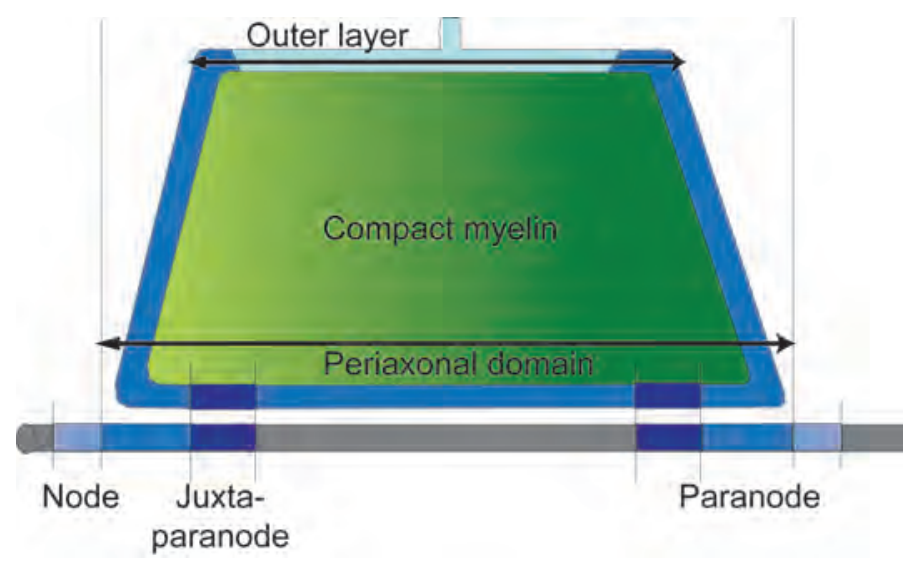

Figure 1.4: Schematic view of polarized myelin along the radial direction. Apart from the polarization along the lateral side, myelin is also radially polarized. The two major radial domains include the outer layer (close to the cell body of the oligodendrocyte) and the periaxonal domain (close to the axon). Figure adapted from (Salzer, 2003). 


\section{Periaxonal and abaxonal domains}

In addition to this longitudinal polarity, myelin is also asymmetric along the radial dimension (Figure 1.4). The inner most layer of myelin (also called periaxonal region) runs parallel to the axon and is non-compacted just like the paranodes and juxtaparanodes. Moreover, it harbors specific set of molecules like MAG and Necl4 that are absent from the outer layers (Maurel et al., 2007; Quarles, 2007; Spiegel et al., 2007). Similarly, the outer most layer (also called abaxonal domain) contains proteins like MOG.

The examples given above clearly illustrate the importance of axo-glia contacts in establishment of cellular polarity and how this polarity in turn orchestrates the formation of functional domains within myelin and axons.

\subsection{Cellular polarization}

In biological terms, a membrane is polar when certain components are distributed asymmetrically along its axis (Cove, 2000). The theme of cell polarization is common not just among different cell types, but also between different organisms. Ranging from simple organisms like prokaryotic bacteria or eukaryotic yeast to complex multicellular tissues, polarization is important for many cellular functions like proliferation, differentiation and morphogenesis.

\subsubsection{Mechanisms of cellular polarization}

Considering the wide diversity between the cell and tissue type that exhibit polarity, the assumption would be that mechanism of asymmetry establishment is unique for a given cell type. However, despite enormous variety amongst the cell types, polarization is executed via common, yet conserved fundamental mechanisms. The major ones include segregation and trafficking to a defined domain, preferential retention after non-specific targeting and selective endocytosis (transcytosis) after polarized delivery. 


\section{Targeted delivery model}

In this model, there is polarized delivery of components to a specific domain. Components with similar sorting signals are pre-sorted at the Golgi and bud into common carrier vesicles, which then fuse with the target compartment. Epithelial cells use this mechanism to target proteins specifically to the apical or basolateral domain. In these cells, the apical domain faces the external space, while the basolateral domain is surrounded by the cellular matrix. Proteins destined for the basolateral domain have either tyrosine-based (Fölsch, 2008) or dileucine-based (Hunziker and Fumey, 1994) targeting motifs in their cytosolic domains. In the Golgi, these motifs are recognized by specific adaptor proteins, for example AP-4 in case of MDCK cells (Simmen et al., 2002) and AP-1, AP-1B for LLC-PK1 cells (Fölsch et al., 1999). Sorting at the Golgi further requires GTPases Arf, Arl and Rab family of proteins. All these GTPases continuously cycle between inactive (GDP bound) and active state (GTP bound). In comparison to basolateral domain, apical membrane proteins have sorting signals in the transmembrane or extracellular domain (Schuck and Simons, 2004).

\section{Diffusion retention model}

In the diffusion retention model, proteins destined for distinct membrane domains are packed into common vesicular carriers and diffuse freely between the two domains. However, preferential retention in one of the domains leads to their further enrichment. For instance, in neurons, the synaptic vesicle protein VAMP2 is initially delivered to both axons and somatodendritic compartment. However, selective endocytosis in dendrites at the later stages enriches the protein in axons (Sampo et al., 2003).

\section{Transcytosis}

In this pathway, different proteins first arrive together at one common domain. Thereafter, the non-resident proteins are selectively endocytosed. In hepatocytes, polarized epithelial cells of liver, proteins are first delivered to the basolateral side. Apical proteins are then preferentially endocytosed and delivered via vesicular trafficking to the apical domain (Wang and Boyer, 2004). The vesicular trafficking 
involves early endosomes (Shivas et al., 2010).

\section{Orientation cues for the polarization machinery}

Cell polarity can also be generated and maintained by a set of conserved intracellular protein complexes. PAR, crumbs and scribble are the three major polarity complexes that provide specific orientation cues for different components. In case of epithelial cells PAR and crumbs localize to the apical membrane, while scribble is mainly present in the basolateral membrane. Activated cdc42 binds and thereby recruits par6 to the apical membrane. Par6 then associates with par3 and aPKC to form the PAR complex. It is important to note that aPKC also phosphorylates $\operatorname{lgl}$ and keeps it inactive on the apical membrane. Since there is no aPKC on the basolateral side, lgl is active and in turn interacts with scribble and establishes basolateral polarity (Hutterer et al., 2004; Humbert et al., 2006; Bryant and Mostov, 2008). Mis-localization of either of these proteins disrupts polarity. The mechanisms by which these proteins promote cellular polarity are currently unknown.

\section{Diffusion barriers}

Diffusion is a physical process in which random thermal agitation of particles causes their mixing. If not hindered, biomolecules can freely diffuse in cell. A diffusion barrier is a structure/fence which prevents this intermixing by restricting the random movements to well defined areas. These barriers play a very important role in the maintenance of cellular polarity . Once different domains have been established in a membrane (via distinct vesicular trafficking), diffusion barriers act as molecular fences restricting the movements of components between the two domains. There are many well-established examples of diffusion barriers in a variety of cell types (Figure 1.5). In case of epithelial cells, tight junctions prevent the mixing of lipids in the outer leaflet and transmembrane proteins between apico-basolateral domains. The septin diffusion barrier, existent at the base of the primary cilium, prevents diffusion of ciliary components into the underlying plasma membrane ( $\mathrm{Hu}$ et al., 2010). Another lateral diffusion barrier is present in the plasma membrane at the axon initiation segment (Rasband, 2010b). The barrier in AIS is made up of a set of proteins that include actin, AnkG, Nfas186, NrCAM and voltage dependent sodium 
channels. This barrier not only restricts the diffusion of membrane proteins, but also soluble proteins as well as certain lipids (Winckler et al., 1999; Nakada et al., 2003; Song et al., 2009).

A

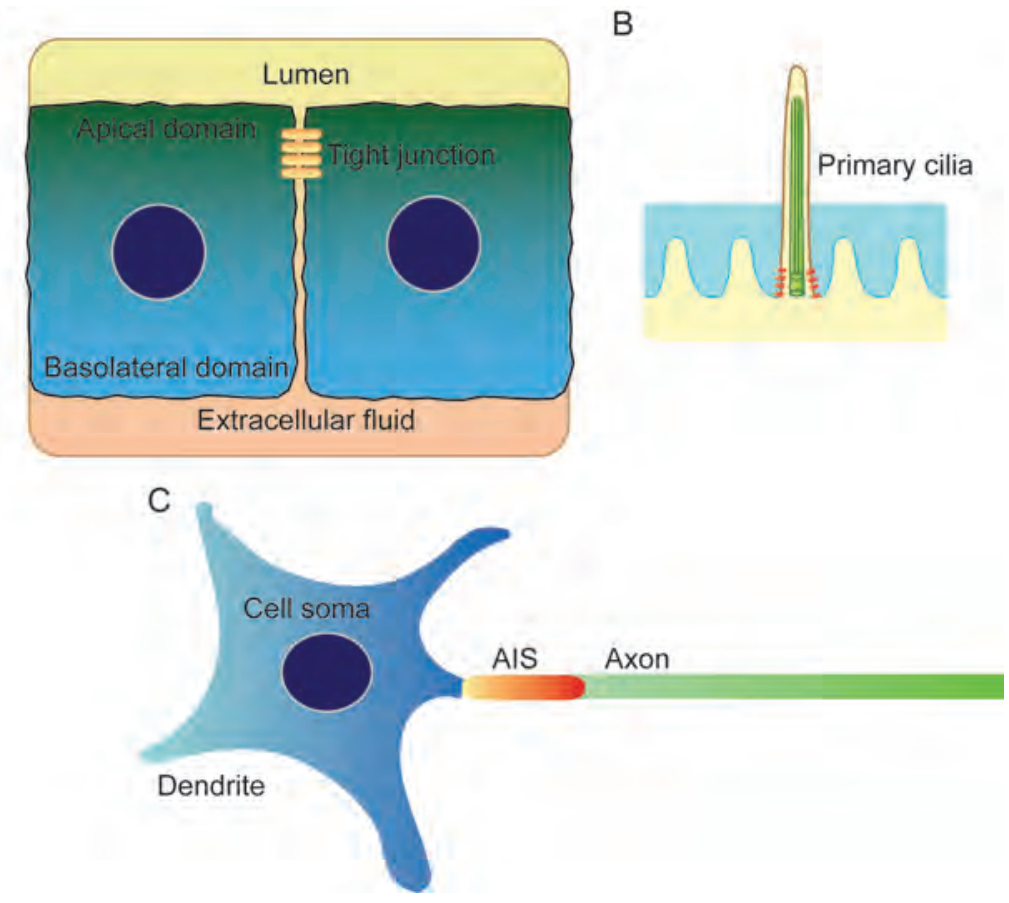

Figure 1.5: Examples of lateral diffusion barriers. (A) Tight junctions in epithelial cells separate apical membrane domain from the basolateral side (B) Septin diffusion barrier (orange) separates membrane protein from cilliary components. (C) Axon initiation segment (AIS) compartmentalize neurons into axons and dendrites. Modified from (Caudron and Barral, 2009).

Diffusional barriers can functionally play dual roles. First, they maintain domain identity and second they serve as scaffolds. For example, in budding yeast septins serve as scaffolds in order to recruit and possibly stabilize the contractile machinery. At later stages, they confine membrane associated factors to the cleavage site via diffusion barrier formation (Caudron and Barral, 2009).

Composition and assembly process for some diffusion barriers is well described. At the axon initiation segment, a dense network of actin cytoskeleton anchors various transmembrane proteins. Anchored membrane proteins function as pickets and re- 
strict the mobility of lipids and transmembrane proteins from the axonal membrane into the soma or dendrites (Nakada et al., 2003). AnkG was also shown to be directly involved in the barrier maintenance through knockdown experiments (using short-hairpin RNA) in cultured mature hippocampal neurons. AIS disassembled in the absence of AnkG and one of processes acquired characteristics of both axons and dendrites (Hedstrom et al., 2008; Rasband, 2010b). Recently, it has been shown that AnkG is clustered at the AIS by distal cytoskeleton comprised of AnkB, $\alpha$ II-spectrin and $\beta$ II-spectrin (Galiano et al., 2012).

\subsubsection{Mechanisms of myelin membrane polarization}

As stated above, apart from the compact and non-compact domains, myelin membrane is polarized along the radial as well as lateral direction. Several key polarity mechanisms of domain establishment within PNS myelin have been demonstrated. Radially, abaxonal domain is similar to the basolateral domain of epithelial cells. On the other hand, apical membrane regulators like pals1 (Protein associated with lin seven 1) and par3 are enriched in the periaxonal domain (Pereira et al., 2011). Pals1 knock down with short hairpin RNAs (shRNAs) resulted in reduced surface expression of MAG and E-cadherin in rat epithelial cells. In myelinating schwann cell-axonal co-cultures, polarized distribution of trafficking machinery was abolished in the absence of pals1 (Ozçelik et al., 2010).

Diffusion barriers also have been shown to exist along the myelinated domains and maintain their identity. For example, axo-glia contacts at the paranodes restrict diffusion of $\mathrm{Na}^{+}$to the nodal areas (Pedraza et al., 2001; Rios et al., 2003). Furthermore, they also separate nodes from the juxtaparanodes, which harbor $\mathrm{K}^{+}$channels. Contactin/caspr and Nfas155 complex plays an important role in this barrier formation. In neurofascin knock-out mice, paranodal domains are disrupted and the distribution of $\mathrm{Na}^{+}$channels is diffuse (Sherman et al., 2005). Furthermore, ablation of Nfas155 in adult myelinating glia via PLP-CreER recombinase disrupted the fencing and caused disorganization of axonal domains (Pillai et al., 2009). 
Another example includes the autaptic tight junctions present along the radial component in the compact myelin. Claudin-11 forms these junctions and promotes electrical isolation of the myelin lamella from the extracellular space. Claudin-11 null mice form ultrastructurally normal myelin without the assembly of radial component (Gow et al., 1999). However, nerve conduction is severely slowed down emphasizing the need for the claudin-11 barrier (Devaux and Gow, 2008).

\subsection{Aims of the work}

Whereas most of the previous studies have been dedicated to elucidate the mechanisms of radial and lateral compartmentalization of myelin, the principles which segregate bulk of the compact myelin from the non-compact myelin remain poorly understood. Compact myelin has a very specific lipid and protein composition, which is distinct from the non-compact areas. It is immensely enriched in lipids and contains a low proportion of mostly low molecular weight proteins. This unique composition is required for the insulation of axons. The first aim of the work was to identify the mechanism(s), which generate two major domains within myelin, namely the compact and non-compact myelin. We used the model system of cultured oligodendrocytes that establish these two polarized domains in two-dimension. This model system allowed us to visualize the myelin domains via light microscopy. Our goal was to identify the players which generate oligodendroglial polarity in two-dimension. The question was whether compact and non-compact domains are established via distinct vesicular trafficking or lateral heterogeneity is established after common sites of vesicular deliver. Diffusion barriers are known to play an important role in the maintenance of asymmetry. Therefore, we also wanted to investigate whether a diffusion barrier prevents intermixing of the components between domains. Further, we aimed to identify conditions in which proteins missort into one or the other domain. To address these questions, we employed a variety of cell biological, biochemical, biophysical and molecular biology approaches. We were also interested in validating our key findings in the in vitro minimal component assays as well as in vivo, using mice models. 


\section{Materials and Methods}

\section{$2.1 \quad$ Materials}

\subsubsection{Antibodies}

Primary antibodies used in this study are listed in 2.1.

Table 2.1: Antibodies used in this study: IF (Immunofluorescence), WB (Western blot), $r$ :rabbit (affinity purified or serum), m:mouse (monoclonal).

\begin{tabular}{lll}
\hline Target & Application & Reference \\
\hline$m$-anti-CNPase & IF (1:200), WB (1:500) & Sigma-Aldrich \\
$m$-anti-GalC (clone MAB342) & IF (1:200) & Millipore \\
$r$-anti-GFP & IF (1:1000), WB (1:5000) & Invitrogen \\
$r$-anti-HA & IF (1:500), WB (1:1000) & Abcam \\
$m$-anti-MAG (clone 513) & IF (1:200) & Millipore \\
$m$-anti-MBP & IF (1:1000) & Sternberger \\
$r$-anti-MBP & IF (1:300), WB (1:2000) & DakoCytomation \\
$r$-anti-MOG (clone 8-18-C5) & IF (1:100), WB (1:500) & Millipore \\
$r$-anti-Myc & IF (1:500), WB (1:1000) & Sigma-Aldrich \\
$m$-anti-Myc (clone 9E10) & IF (1:500), WB (1:5000) & Sigma-Aldrich \\
$m$-anti-O1 (IgM) & IF (1:50) & (Sommer and Schachner, \\
& & 1981) \\
$r$-anti-Septin 7 & IF (1:100) & Immuno-Biological Lab- \\
$m$-anti- $\alpha$ tubulin & IF (1:2000), WB (1:5000) & Sigma-Aldrich \\
$m$-anti-polyglut-tubulin (IgM) & IF (1:1000) & Sigma-Aldrich \\
\hline
\end{tabular}


Antibodies were obtained from Abcam (Cambridge, UK), DakoCytomation (Carpinteria, CA, USA), Immuno-Biological Laboratories (Hamburg, Germany), Invitrogen (Darmstadt, Germany), Millipore (Billerica, MA, USA), Sigma-Aldrich (Munich, Germany), Sternberger (Lutherville, MD, USA) and Stressgen Bioreagents (Victoria, Canada). Secondary fluorophore ( $\mathrm{Cy} 3$ or $\mathrm{Cy} 5)$ and peroxidase-conjugated antibodies (anti-mouse $\operatorname{IgG}$, anti-mouse $\operatorname{IgM}$ and anti-rabbit) were purchased from Dianova (Hamburg, Germany). Anti-rabbit, anti-mouse IgG and anti-mouse IgM secondary antibodies coupled to Alexa-488 fluorophore were purchased from Invitrogen (Darmstadt, Germany). Alexa-488 and Cy5-conjugated secondary antibodies were used at a dilution of 1:100. Cy3-conjugated anti-rabbit antibodies were diluted 1:1000. Cy3-conjugated anti-mouse antibodies were used at 1:300 dilution.

\subsubsection{Chemicals, Enzymes and Kits}

Ionomycin was purchased from Calbiochem (Darmstadt, Germany), rapamycin from Sigma-Aldrich (Munich, Germany), and sphingosine from Avanti Polar Lipids (Alabaster, AL, USA). 1,2-dioleoyl-sn-glycero-3-phosphoethanolamine (PE), porcine brain L- $\alpha$-phosphatidylinositol-4,5-bisphosphate (PIP2), L- $\alpha$-phosphatidyl choline $(\mathrm{PC})$, porcine brain sulfatide and porcine brain L- $\alpha$-phosphatidylserine (PS) were obtained from Avanti Polar Lipids as chloroform stocks. Cholesterol and chicken egg yolk sphingomyelin (eSM) were obtained from Sigma-Aldrich (Munich, Germany). Lissamine $^{\mathrm{TM}}$ rhodamine B 1,2-dihexadecanoyl-sn-glycero-3- phosphoethanolamine (rhodamine-DHPE), Texas Red DHPE, concanavalin A (ConA) coupled to Alexa594, wheat germ agglutinin (WGA) coupled to Tetramethylrhodamine, cell mask orange, Alexa-488 $\mathrm{C}_{5}$ maleimide and tris(2-carboxyethyl)phosphine (TCEP) were bought from Invitrogen (Munich, Germany). Protease inhibitor cocktail Complete Mini (EDTA-free) was acquired from Roche Applied Science (Mannheim, Germany). Other chemicals were obtained from either Sigma-Aldrich (Munich, Germany), Invitrogen (Munich, Germany), Roth (Karlsruhe, Germany), Merck (Darmstadt, Germany) or Applichem (Darmstadt, Germany), unless otherwise stated. Each chemical was either of analytical purity or cell culture grade. Cell culture media and supplements like Dulbecco's modified eagle's medium (DMEM) with high glucose, 
Hank's balanced salt solution (HBSS), GlutaMAX ${ }^{\mathrm{TM}}$, Pen/Strep, B-27 supplement, sodium pyruvate, $0.05 \%$ trypsin-EDTA and $0.25 \%$ trypsin-EDTA were purchased from Gibco (Invitrogen, Darmstadt, Germany). DMEM with $4.5 \mathrm{~g} / \mathrm{L}$ glucose, Horse serum (HS), Fetal calf serum (FCS) and Dulbecco's Phosphate buffer saline (PBS) were obtained from PAA (Cölbe, Germany). Consumables were purchased from Falcon (Becton Dickinson Labware Europe, Le Pont De Claix, France) and Eppendorf (Eppendorf AG, Hamburg, Germany). Culture flasks $\left(75 \mathrm{~cm}^{2}\right.$ and $\left.175 \mathrm{~cm}^{2}\right)$, dishes $(10 \mathrm{~cm}$ and $15 \mathrm{~cm}$ ) and plates (6-well, 12-well and 24-well) were obtained from Greiner Bio-One (Greiner Bio-One GmbH, Frickenhausen, Germany).

\section{Buffers and Enzymes}

The buffers and solutions used in the study are listed in Table 2.2. Enzymes used in the study are listed in Table 2.3 and were mainly obtained from Fermantas (St. Leon-Rot, Germany), New England Biolabs (NEB GmbH, Frankfurt, Germany), Promega (Mannheim, Germany) and Finnzymes (Espoo, Finland).

Table 2.2: Buffers and solutions used in the study

\begin{tabular}{|c|c|}
\hline Buffers and solutions & Ingrediants \\
\hline $\begin{array}{l}\text { Phosphate buffer saline } \\
\text { (PBS) }\end{array}$ & $150 \mathrm{mM} \mathrm{NaCl}, 20 \mathrm{mM} \mathrm{Na}_{2} \mathrm{HPO}_{4}$, pH 7.4 \\
\hline Krebs-Ringer's solution & $\begin{array}{l}120 \mathrm{mM} \mathrm{NaCl}, 4.7 \mathrm{mM} \mathrm{KCl}, 1.2 \mathrm{mM} \mathrm{CaCl}, 0.7 \mathrm{mM} \\
\mathrm{MgSO}_{4}, 10 \mathrm{mM} \text { glucose and } 10 \mathrm{mM} \text { HEPES, } \mathrm{pH} 7.4\end{array}$ \\
\hline Mowiol & $\begin{array}{l}6 \text { g glycerol AR, } 2.4 \text { g Mowiol 4-88 (Calbiochem), } 6 \mathrm{~mL} \\
\mathrm{H}_{2} \mathrm{O}, 12 \mathrm{~mL} 0.2 \mathrm{M} \text { Tris } \mathrm{pH} 8.5\end{array}$ \\
\hline Blocking solution & $\begin{array}{l}2 \mathrm{~g} \text { BSA (Applichem), } 2 \mathrm{~mL} \text { FCS (PAA), } 2 \mathrm{~mL} \text { Fish } \\
\text { gelatin (Sigma-Aldrich), } 10 \mathrm{~mL} 10 \times \text { PBS, final volume } \\
100 \mathrm{~mL} \text { with distilled } \mathrm{H}_{2} \mathrm{O}\end{array}$ \\
\hline LB media & $25 \mathrm{~g} / \mathrm{L}$ LB-powder (Applichem) in distilled $\mathrm{H}_{2} \mathrm{O}$ \\
\hline $1 \times$ Lysis buffer & $\begin{array}{l}150 \mathrm{mM} \mathrm{NaCl}, 1 \mathrm{mM} \text { EDTA, } 1 \% \text { TritonX-100 and } 20 \mathrm{mM} \\
\text { Tris- } \mathrm{HCl} \mathrm{pH} 7.5\end{array}$ \\
\hline
\end{tabular}


Table 2.3: Enzymes used in this study

\begin{tabular}{lll}
\hline Enzyme & Application & Reference \\
\hline Restriction enzymes & DNA digest & NEB or Fermentas \\
T4 ligase & Ligation of DNA fragments & Fermentas \\
Taq DNA polymerase & Genotyping & Promega \\
Phusion DNA polymerase & Polymerase chain reaction & Finnzymes \\
Antartic phosphatase & $5^{\prime}$ phosphate removal & NEB \\
T4 polynucleotide kinase & $5^{\prime}$ phosphorylation & NEB \\
\hline
\end{tabular}

\section{Kits}

Kits availed in the study are listed in Table 2.4 and were used together with the supplied buffer solutions in accordance with manufacturer's guidelines.

Table 2.4: Commercial kits used in this study

\begin{tabular}{lll}
\hline Kit & Application & Reference \\
\hline NucleoSpin Plasmid kit & DNA isolation, small scale & Macherey-Nagel \\
NucleoBond ${ }^{\mathbb{R}}$ Xtra EF & DNA isolation, medium scale & Macherey-Nagel \\
NucleoSpin Extract II kit & DNA extraction from agarose gels & Macherey-Nagel \\
Nucleospin $^{\circledR}$ Gel & DNA extraction from gel & Macherey-Nagel \\
and PCR Clean-Up & and PCR clean up & \\
Lipofectamine $^{\text {TM }}$ 2000 & transient transfection, primary cells & Invitrogen \\
TransIT(R)-LT1 & transient transfection, cell lines & Mirus Biologicals \\
Pierce ${ }^{\circledR}$ BCA protein assay & protein quantification & ThermoFischer \\
Invisorb spin tissue mini kit & tail DNA extraction & Invitek \\
Bio-Rad Protein Assay & protein quantification & Bio-Rad \\
\hline
\end{tabular}

\subsubsection{Mammalian cell lines and bacterial strains}

The mammalian cell lines and the bacterial strains used in the study are listed in Table 2.5. 
Table 2.5: Cell lines and bacterial strains employed in the study

\begin{tabular}{|c|c|c|}
\hline Cells & Description & Reference \\
\hline PtK2 & Male rat kangaroo kidney epithelial cells & (Eggeling et al., 2009) \\
\hline Oli-neu & Murine oligodendroglial precursor cell line & (Jung et al., 1995) \\
\hline & $\mathrm{F}^{-} \quad$ \$80lacZ1 $($ lacZYA- $\arg \mathrm{F}) \mathrm{U} 169 \quad$ recA1 end $\mathrm{A} 1$ & \\
\hline $\mathrm{DH} 5 \alpha$ & $\begin{array}{l}\text { hsdR17 }\left(\mathrm{r}_{\mathrm{k}}^{-}, \mathrm{m}_{\mathrm{k}}{ }^{+} \text {phoA sup E44 thi-1 gyr A96 relA1 }\right. \\
\lambda^{-}\end{array}$ & Invitrogen \\
\hline
\end{tabular}

\subsection{Methods}

\subsubsection{Molecular biology and biochemical methods}

\section{Molecular Cloning}

For molecular cloning standard methods of restriction digestion, ligation and transformation (via heat shock) into chemically competent E.coli $\mathrm{DH} 5 \alpha^{\mathrm{TM}}$ competent cells were used. Clones were screened through restriction digestion of plasmid 'miniprep' followed by sequencing and large scale preparation of DNA via 'midi-prep' using commercial kits. DNA primers were either ordered from AGCT lab (Central facility at the Max Planck Institute of Experimental Medicine, Göttingen, Germany) or biomers.net (Ulm/Donau, Germany). MBP mutant genes (with multiple point mutations) were ordered from Genscript (New Jersey, USA). The DNA constructs cloned in the study are listed in Table A. The $5^{\prime}-3^{\prime}$ sequence of primers employed for the study are included in Table A.

\section{Protein determination}

Protein concentration was determined according to Bradford (Bradford, 1976). Protein standards were prepared in the range of 1-10 $\mu \mathrm{g}$ using bovine serum albumin (BSA, Applichem). Standards were further diluted in $200 \mu \mathrm{L} \mathrm{H}_{2} \mathrm{O}$ and then mixed with $800 \mu \mathrm{L}$ bradford solution (Bio-Rad). The samples were incubated at RT for 5 min followed by absorbance measurements at $595 \mathrm{~nm}$ wavelength using a photometer 
(Bio-Rad). From the standard curve (linear trace), unknown protein concentrations for the test samples were interpolated.

BCA assay (Smith et al., 1984) was used to quantify the total protein content of cell lysates. This assay was performed using Pierce BCA Protein Assay Kit (Thermo Scientific) according to the manufacturer's manual.

\section{SDS-PAGE and Western blotting}

Samples were separated on 10\% denaturing Tris-SDS polyacrylamide gel electrophoresis system as described before (Laemmli, 1970). The resolving gel contained $10 \%$ acrylamide/bis-acrylamide (37.5:1, Bio-Rad), $375 \mathrm{mM}$ Tris (pH 8.5), 0.1\% SDS, $0.05 \%$ ammonium per sulphate (APS) and 0.005\% TEMED (N,N,N $N^{\prime} \mathrm{N}^{\prime}$ Tetramethyethylenediamine). The stacking gel had 4\% acrylamide/bis-acrylamide (37.5:1, Bio-Rad), 125 mM Tris (pH 6.8), 0.1\% SDS, 0.05\% ammonium persulphate (APS) and $0.005 \%$ TEMED. Before loading, samples were boiled in the sample buffer (50 $\mathrm{mM}$ Tris $\mathrm{pH}$ 6.8; $10 \%$ glycerol, 2 mM EDTA, 2\% SDS, $0.05 \%$ bromophenol blue and $144 \mathrm{mM} \beta$-mercaptoethanol) for $10 \mathrm{~min}$ at $70^{\circ} \mathrm{C}$. For each gel, $5 \mu \mathrm{L}$ of pre-stained protein ladder (Fermentas) was loaded into one of the lanes. Separation was performed in a continuous buffer system containing Tris-glycine electrophoresis buffer (25 mM Tris, $192 \mathrm{mM}$ glycine and 0.1\% SDS).

Western blotting was done as described before (Towbin et al., 1979). Briefly, proteins were transferred onto a Protran ${ }^{\circledR}$ (Whatman GmbH, Dassel, Germany) via a semidry procedure in the transfer buffer (25 mM Tris at pH 7.4, $192 \mathrm{mM}$ glycine and $2 \%$ methanol). For the transfer, $100 \mathrm{~V}$ was applied for $1 \mathrm{hr}$ in a Bio-rad PowerPac 1000 blotting system. The membranes were then blocked with $4 \%$ non-fat dried milk powder (Sigma-Aldrich), prepared in PBST (0.1\% Tween-20 in PBS), for 30 min followed by overnight incubation in primary antibodies (diluted appropriately in PBST). Following primary antibody incubation, blots were washed three times, 10 min each, in PBST and then incubated with secondary antibodies (peroxidase conjugated, diluted 1:2000 in PBST) for $1 \mathrm{hr}$ at RT. Afterwards, blots were washed three times with PBST (10 min each). Finally, protein bands were detected on X-ray films (CL-XPosure ${ }^{\mathrm{TM}}$, Thermo Scientific, Pierce) using enhanced chemiluminescence 
(ECL, Thermo Scientific, Pierce) as a substrate for peroxidase on Kodak imaging station.

Alternatively, gels were directly stained with Coomassie brilliant blue G-250 (Fairbanks et al., 1971). For staining, stacking gel was discarded and the resolving gel was washed once with water. Next, the gels were stained with solution A $(25 \%$ isopropanol, $10 \%$ acetic acid and $0.025 \%$ coomassie brilliant blue) by heating for 30 sec in a microwave. Gels were then destained in solution B (10\% acetic acid, $10 \%$ isopropanol and $0.005 \%$ coomassie brilliant blue) followed by solution C (10\% acetic acid and $0.002 \%$ coomassie brilliant blue), each time for 30 sec in a microwave. Finally, the gels were destained with $10 \%$ acetic acid overnight, washed in water and scanned.

\subsubsection{Expression and purification of proteins}

To produce GFP with N-terminally attached lipid binding regions R1-3 of NHE3 (Alexander et al., 2011), Escherichia coli strain TOP10F' was transformed with the respective constructs pSF1622 to pSF1624 and grown at $25^{\circ} \mathrm{C}$ to $\mathrm{OD}_{600}=3.0$ in TB medium (supplemented with $50 \mathrm{mg} / \mathrm{mL}$ kanamycin). The culture was induced with $0.15 \mathrm{mM}$ isopropyl $\beta$-D-1-thiogalactopyranoside (IPTG), warmed to $37^{\circ} \mathrm{C}$ and further shaken over night. Before cell harvest, $1 \mathrm{mM}$ phenylmethylsulfonylfluoride (PMSF) and $5 \mathrm{mM}$ EDTA were added directly to the culture. Cells were resuspended in buffer $\mathrm{HS}(1.3 \mathrm{M} \mathrm{NaCl}, 220 \mathrm{mM}$ Tris-HCl pH 7.5, $11 \mathrm{mM} \mathrm{MgCl}, 10$ mM imidazole, $2.2 \mathrm{mM}$ EDTA, $10 \mathrm{mM}$ DTT) and lysed by sonification. After centrifugation for $1 \mathrm{hr}$ at 37,000 $\mathrm{rpm}$, the cleared lysate was applied to a nickel-chelate matrix equilibrated with buffer HS. After extensively washing with buffer HS and buffer HNS (20 mM HEPES, $100 \mathrm{mM} \mathrm{NaCl}, 250 \mathrm{mM}$ sucrose, $5 \mathrm{mM}$ DTT, final pH 7.3), bound protein was eluted by incubation with SUMO protease (20 nM) for 15 min at RT.

H14-TEV-MBP14/MBP F $\rightarrow$ S-Cys was expressed from pSF1625 in E. coli strain BLR harboring plasmid pRil. Cultures were grown in TB medium supplemented with $50 \mathrm{mg} / \mathrm{mL}$ kanamycin and $37 \mathrm{mg} / \mathrm{mL}$ chloramphenicol at $37^{\circ} \mathrm{C}$ to $\mathrm{OD}_{600}=$ 
6.0, induced with $1 \mathrm{mM}$ IPTG and further shaken at $37^{\circ} \mathrm{C}$ for 6 hrs at $37^{\circ} \mathrm{C}$. The protein was purified essentially as described for the Nsp1 FG/FxFG repeat domain (Eisele et al., 2010). Before cell harvest, $1 \mathrm{mM} \mathrm{PMSF}$ and $5 \mathrm{mM}$ EDTA were added directly to the culture. Cells were resuspended in 8.3 M guanidinium-hydrochloride (Gua-HCl) containing $2 \mathrm{mM}$ EDTA and $20 \mathrm{mM}$ DTT and lysed by a single round of freezing and thawing. After centrifugation for $1 \mathrm{hr}$ at 37,000 rpm, the cleared lysate was supplemented with $100 \mathrm{mM}$ Tris- $\mathrm{HCl} \mathrm{pH} 8.5$ and $1 \mathrm{mM}$ imidazole; and applied to a nickel-chelate column. The column was washed with $7.5 \mathrm{mM}$ Gua$\mathrm{HCl}, 100 \mathrm{mM}$ Tris/HCl (pH 8.5), $1 \mathrm{mM}$ EDTA, $1 \mathrm{mM}$ imidazole followed by a second wash step with $8 \mathrm{M}$ urea, $20 \mathrm{mM}$ Tris-HCl pH 8.0, $1 \mathrm{mM}$ EDTA, $1 \mathrm{mM}$ imidazole. Bound protein was eluted with the same buffer supplemented with 500 mM imidazole, diluted 1:3 with water and applied to a thiopyridine-activated, SHreactive matrix. The matrix was washed with $6 \mathrm{M}$ Gua-HCl, $20 \mathrm{mM}$ Tris/HCl $(\mathrm{pH}$ 8.0), $1 \mathrm{mM}$ EDTA, $1 \mathrm{mM}$ imidazole, and eluted with $6 \mathrm{M}$ Gua-HCl, $20 \mathrm{mM}$ Tris- $\mathrm{HCl}$ pH 7.5, $10 \mathrm{mM}$ DTT, applied to a preparative C18 reverse phase HPLC column, eluted with increasing concentrations of acetonitrile in $0.15 \%$ TFA, and lyophilized.

\subsubsection{Biochemical assays}

\section{Cell lysis}

Primary cells $(\sim 500,000$ cells per well) or PtK2 cells ( $80 \%$ confluent) were grown in 6-well plates for the desired time periods. After aspirating the medium, cells were washed once with PBS and scraped in $100 \mu \mathrm{L}$ of lysis buffer on ice and further incubated for $10 \mathrm{~min}$ (on ice). Following incubation in the lysis buffer, the samples were centrifuged (pre-cooled bench top centrifuge) at 20,817 g for $10 \mathrm{~min}$. Pellet was discarded and protein concentration in the supernatant was determined using bradford assay.

\section{Myelin membrane preparation}

Myelin membrane was prepared from adult mice as described before (Norton and Poduslo, 1973). Briefly, mice between 3-10 weeks were sacrificed, whole brains were removed followed by removal of meninges. Each brain was individually homogenized 
via sonication in $1 \mathrm{~mL}$ of $0.32 \mathrm{M}$ sucrose (supplemented with protease inhibitor cocktail). Homogenized brain suspension was further diluted with the addition of $2 \mathrm{~mL}$ of cold water. $1.5 \mathrm{~mL}$ of diluted homogenate was layered on top of a two-step gradient consisting of $3.5 \mathrm{~mL}$ each of $0.85 \mathrm{M}$ lower sucrose phase and $0.32 \mathrm{M}$ upper sucrose phase (each sucrose solution was buffered with $10 \mathrm{mM}$ HEPES, pH 7.5 and supplemented with $5 \mathrm{mM}$ EDTA) in $14 \times 89 \mathrm{~mm}$ ultra-clear centrifugation tube (Beckman Coulter GmbH, Krefeld, Germany). The gradients were set up in multiple tubes at a time and tubes were centrifuged at 75,000 g for 30 min (Beckman SW41Ti rotor). The interface between $0.85 \mathrm{M}$ and $0.32 \mathrm{M}$ sucrose was collected and diluted up to 10 times with ice-cold water and pelleted via low speed centrifugation at 12,000 g for $30 \mathrm{~min}$. The pellet was again resuspended in ice-cold water and recentrifuged as described above. This preparation gives so called 'crude myelin'. For pure myelin membrane preparation, the crude myelin was once again loaded onto the density gradient followed by two washing steps with ice cold water as described above. Finally, the supernatant was discarded and the pellet was resuspended in PBS. Protein concentration was determined using either bradford or BCA assay.

\section{Lipid extraction from isolated myelin}

For determining lipid-to-protein ratios for the wild type (WT) and shiverer (Shiv) myelin, protein concentration was determined in the crude myelin samples using either Bradford or BCA assay. Equal protein amounts from WT and Shiv myelin were subjected to lipid extraction protocol as described earlier (Folch et al., 1957). Briefly, $1 \mathrm{~mL}$ of Methanol:Chloroform (1:2) was added to $12 \mu \mathrm{g}$ of a sample (containing $500 \mathrm{ng}$ of lanosterol as internal standard to quantify cholesterol amount) and the samples were gently rotated at $4^{\circ} \mathrm{C}$ for 30 min. Afterwards, $0.3 \mathrm{~mL}$ of $\mathrm{H}_{2} \mathrm{O}$ was added to each sample followed by further incubation on ice for $15 \mathrm{~min}$. Samples were then centrifuged at 20,800 g, $4^{\circ} \mathrm{C}$ for 15 min in a tabletop centrifuge (Eppendorf AG, Hamburg, Germany). The lower phase was collected, dried under the stream of nitrogen, resuspended in chloroform $(50 \mu \mathrm{L})$ and subjected to gas chromatography. Cholesterol analysis was performed with an Agilent (Waldbronn, Germany) 6890 gas chromatograph filled with a capillary HP-5 column $(30 \mathrm{~m} \times 0.32 \mathrm{~mm} ; 0.25$ $\mu \mathrm{m}$ coating thickness; J\&W Scientific, Agilent). Helium was used as a carrier gas 
$(1 \mathrm{~mL} / \mathrm{min})$. The temperature was $200^{\circ} \mathrm{C}$ for $1 \mathrm{~min}, 200-325^{\circ} \mathrm{C}$ at $20 \mathrm{~K} / \mathrm{min}$ and $325^{\circ} \mathrm{C}$ for $7.5 \mathrm{~min}$.

Preparation of methyl esters of fatty acids (FAMEs) for analysis by gas chromatography/flame ionization detection (GC/FID) was performed as described before (Ren et al., 1993). For acidic hydrolysis, $1 \mathrm{~mL}$ of a methanolic solution containing $2.75 \%$ (v/v) $\mathrm{H}_{2} \mathrm{SO}_{4}(95-97 \%)$ and $2 \%$ (v/v) dimethoxypropane was added to $6 \mu \mathrm{g}$ of each of the myelin samples. For the quantification of the total lipid content, $1 \mu \mathrm{g}$ of triheptadecanoate standard was added and the sample was incubated for $1 \mathrm{hr}$ at $80^{\circ} \mathrm{C}$. To extract the resulting FAMEs, $200 \mu \mathrm{L}$ of saturated aqueous $\mathrm{NaCl}$ solution and 2 $\mathrm{mL}$ of hexane were added. The hexane phase was dried under streaming nitrogen and redissolved with equal volumes of water and hexane. The hexane phase was filtrated from cotton wool soaked in $\mathrm{Na}_{2} \mathrm{SO}_{4}$ and dried under streaming nitrogen. Finally the samples were redissolved in $10 \mu \mathrm{L}$ acetonitrile for $\mathrm{GC}$ analysis that was performed with an Agilent (Waldbronn, Germany) 6890 gas chromatograph fitted with a capillary DB-23 column $(30 \mathrm{~m} \times 0.25 \mathrm{~mm} ; 0.25 \mu \mathrm{m}$ coating thickness; J\&W Scientific, Agilent). Helium was used as carrier gas at a flow rate of $1 \mathrm{~mL} / \mathrm{min}$. The temperature gradient was $150^{\circ} \mathrm{C}$ for $1 \mathrm{~min}, 150-200^{\circ} \mathrm{C}$ at $8 \mathrm{~K} / \mathrm{min}, 200-250^{\circ} \mathrm{C}$ at $25 \mathrm{~K} / \mathrm{min}$ and $250^{\circ} \mathrm{C}$ for $6 \mathrm{~min}$.

\section{Cell based cross-linking assay}

Cell based cross-linking protocol was adapted from the procedure described before (Friedrichson and Kurzchalia, 1998). Briefly, primary oligodendrocytes were grown on 6-well plates $(\sim 500,000$ per well). At 5 DIV, cell medium was aspirated and the wells were washed once with PBS followed by the addition of $0.1-1 \mathrm{mM}$ of the crosslinker disuccinimidyl glutarate (DSG, Thermo Scientific, GmbH, Germany). Cells were incubated with the cross-linker for $30 \mathrm{~min}$ on ice. Cross-linking reaction was quenched by the addition of $1 \mathrm{M}$ Tris- $\mathrm{HCl}, \mathrm{pH} 7.5$ at a final concentration of $20 \mathrm{mM}$ (10 min incubation). Thereafter, the wells were washed with PBS. Cells were subsequently scraped and incubated for $10 \mathrm{~min}$ in $1 \times$ lysis buffer (supplemented with protease inhibitors) and centrifuged at $20,817 \mathrm{~g}\left(10 \mathrm{~min}, 4^{\circ} \mathrm{C}\right)$. Following centrifugation, supernatants were collected; protein concentrations were measured (bradford 
assay) and samples were then processed for SDS-gel electrophoresis.

\section{In vitro cross-linking assay}

Chemical cross-linking was performed between MBP or R3-GFP (control) molecules upon incubation with GUVs composed of PC:PS (2:1 mole\%). Briefly, $340 \mathrm{ng}$ of each, $14 \mathrm{kDa} \mathrm{MBP}$ and R3-GFP were incubated with $20 \mu \mathrm{L}$ GUV solution for $30 \mathrm{~min}$ at RT. Next, various cross-linkers namely DFDNB (1,5-Difuoro-2,4-dinitrobenzene), DSG (Disuccinimidyl glutarate), DMA (Dimethyl adipimidate.2HCl), DMP (Di methyl pimelimidate.2HCl), DMS (Dimethyl suberimidate.2HCl) and DSS (Disuccinimidyl suberate) with a spacer arm of 3.0, 7.7, 8.6, 9.2, 11.0 and $11.4 \AA$ respectively were added at a final concentration of $120 \mu \mathrm{M}$. After 30 min of cross-linking, the reaction was quenched by the addition of $1 \mathrm{M}$ glycine at a final concentration of $50 \mathrm{mM}$ (10 min incubation at RT) and the samples were processed for SDS-PAGE and Western blotting.

\subsubsection{Cell culture}

\section{Primary oligodendrocyte culture}

Oligodendroglial primary cell cultures from post natal day zero (P0) mice were prepared according to a modified protocol (Dubois-Dalcq et al., 1986; Trajkovic et al., 2006). Briefly, P0 mice were sacrificed and forebrains were removed. Following meninges separation, brains ( 5 brains at a time) were incubated with $0.25 \%$ trypsinEDTA for $10 \mathrm{~min}$ at $37^{\circ} \mathrm{C}$. After $10 \mathrm{~min}$ of incubation, trypsin was removed; brains were briefly washed with HBSS followed by addition of $5 \mathrm{~mL}$ BME medium (supplemented with $10 \%$ horse serum, 4 mM GlutaMAX ${ }^{\mathrm{TM}}$ and 50 units/mL each of penicillin and streptomycin) and brain homogenization by passing through a $5 \mathrm{~mL}$ pipette (5-10 times). $1.25 \mathrm{~mL}$ of medium was added per one $75 \mathrm{~mm}^{2}$ tissue culture flask (pre-coated with $100 \mu \mathrm{g} / \mathrm{mL}$ poly-L-Lysine, $\mathrm{M}_{\mathrm{w}}>300000$ ). The mixed cultures were then grown at $37^{\circ} \mathrm{C}$ with $7.5 \% \mathrm{CO} 2$ and $90 \%$ humidity for 7-10 days with intermitant medium changes. After 7-10 days, oligodendrocytes were shaken off from the astrocytic layer, pelleted via centrifugation at $900 \mathrm{~g}$ (10 min) and resuspended in the Super-Sato medium (DMEM with high glucose containing 1x B-27 supplement, 
$2 \mathrm{mM}$ GlutaMAX ${ }^{\mathrm{TM}}, 1 \mathrm{mM}$ sodium pyruvate, $1 \% \mathrm{HS}, 50 \mathrm{units} / \mathrm{mL}$ each of penicillin and streptomycin, $0.5 \mu \mathrm{M}$ triiodothyronine and $0.52 \mu \mathrm{M}$ L-thyroxine). Cells were plated onto $18 \mathrm{~mm}$ coverslips. Shiverer mouse line was maintained by breeding heterozygote carriers of the shiverer allele and genotyping their progeny as described before (Gomez et al., 1990).

\section{PtK2 cells}

PtK2 cells (Eggeling et al., 2009) were kindly provided by C. Eggeling, Max Planck Institute for Biophysical Chemistry, Göttingen, Germany. Cells were cultured in Dulbecco's modified eagle's medium (DMEM with $4.5 \mathrm{~g} / \mathrm{L}$ glucose) with the following additions: 10\% FCS, $4 \mathrm{mM}$ GlutaMAX ${ }^{\mathrm{TM}}, 1 \mathrm{mM}$ pyruvate and 50 units $/ \mathrm{mL}$ each of penicillin and streptomycin. Cells were grown till $90 \%$ confluency on culture flasks at $37^{\circ} \mathrm{C}$ with 5\% CO2 and 90\% humidity. The cells were passaged 1:5 - 1:10 by detaching them from the surface of flask using 0.05\% trypsin-EDTA (incubation at $37^{\circ} \mathrm{C}$ for $5 \mathrm{~min}$ ). Fresh medium supplemented with FCS was added to stop the trypsin reaction. Cells were then split onto pre-sterilized $18 \mathrm{~mm}$ coverslips or onto culture flasks.

\section{Oli-neu cells}

Oli-neu (Jung et al., 1995) were kindly provided by Prof. J. Trotter, University of Mainz, Mainz, Germany. Cells were cultured in SATO medium (DMEM with 3.5 $\mathrm{g} / \mathrm{L}$ glucose supplemented with $2 \mathrm{mM}$ GlutaMAX ${ }^{\mathrm{TM}}, 1 \mathrm{mM}$ sodium pyruvate, $5 \%$ HS, 50 units/mL each of penicillin and streptomycin, 1x insulin-selenium-A supplement, $100 \mu \mathrm{M}$ putrescine dihydrochloride, $0.5 \mu \mathrm{M}$ triiodothyronine and $0.52 \mu \mathrm{M}$ L-thyroxine and $0.2 \mu \mathrm{M}$ progesterone). Cells were grown till $80 \%$ confluency on 10 $\mathrm{cm}$ petri dishes (pre-coated with $33 \mu \mathrm{g} / \mathrm{mL}$ PLL) at $37^{\circ} \mathrm{C}$ with $5 \% \mathrm{CO} 2$ and $90 \%$ humidity. Cells were passaged between 1:3-1:6 by detaching them from the surface of the petri dish using $10 \mathrm{~mL}$ pipette. Cells were then split onto pre-sterilized 18 mm coverslips (PLL coated) or onto petri dishes.

\section{Freezing and thawing of cell lines}

For long-term storage of the cell lines, cells were grown to maximum confluency on 
$10 \mathrm{~cm}$ petri dishes or $75 \mathrm{~cm}^{2}$ cell culture flasks and were either washed off (Oli-neu) or trypsinized (PtK2 cells) as described above. Cells were pelleted by centrifugation at $900 \mathrm{~g}$ for $5 \mathrm{~min}$ and resuspended in $0.5 \mathrm{~mL}$ DMEM. The cell suspension was then added to $0.5 \mathrm{~mL} 2 \times$ freezing medium (40\% FCS, $20 \%$ DMSO in DMEM with 4.5 $\mathrm{g} / \mathrm{L}$ glucose) and mixed gently. Finally, the contents were transferred into freezing vials and the vials were placed in a freezing rack that was first kept at $-80^{\circ} \mathrm{C}$ for 24 hrs (temperature drop rate of $1^{\circ} \mathrm{C} / \mathrm{min}$ ). Frozen cells were later transferred to the liquid nitrogen tank for long term storage.

In order to thaw the cell lines, frozen vials were transferred from liquid nitrogen to $37^{\circ} \mathrm{C}$ water bath. Thawed cell suspension was diluted in $9 \mathrm{~mL}$ fresh cell media. Cells were then pelleted by centrifuging at $900 \mathrm{~g}$ for $5 \mathrm{~min}$. The cell pellet was resuspended in fresh media and plated onto $10 \mathrm{~cm}$ petri dishes or $75 \mathrm{~cm}^{2}$ cell culture flasks (coated with PLL in case of Oli-neu cells).

\subsubsection{Transient Transfection}

\section{Primary Cells}

Pre-treated coverslips were used to culture oligodendrocytes after shaking them off the astrocytic layer. Coverslips were treated with $\mathrm{HCl}$ for $2 \mathrm{hrs}$, washed several times with water, dried and finally sterilized by baking at $200^{\circ} \mathrm{C}$ for $6 \mathrm{hrs} .18 \mathrm{~mm}$ coverslips were placed into 12-well plates and coated with poly-L-Lysine $(100 \mu \mathrm{g} / \mathrm{mL})$ for $1 \mathrm{hr}$ at $37^{\circ} \mathrm{C}$, washed two times with PBS and further incubated with $1 \mathrm{~mL}$ of Super-Sato medium. Cells were plated at a density ranging between 60,000-100,000 per $18 \mathrm{~mm}$ coverslip. Cells were transfected between 3-5 DIV. Lipofectamine ${ }^{\mathrm{TM}} 2000$ kit was used for transient transfections. For one well in a 12-well plate, $1.6 \mu \mathrm{g}$ of plasmid DNA was added to $100 \mu \mathrm{L}$ Opti-MEM and $3 \mu \mathrm{L}$ of lipofectamine ${ }^{\mathrm{TM}} 2000$ reagent was mixed with another $100 \mu \mathrm{L}$ Opti-MEM. Both tubes were incubated for 5 min at RT. The lipofectamine solution was then added to the solution of DNA, mixed gently and further incubated at RT for 24 min. Finally, Opti-MEM-lipofectamine-DNA mixture was gently pipetted on top of cells. The cells were either fixed or imaged 
live, 16-24 hrs after transfection.

\section{Cell lines}

Prior to cell splitting, coverslips were sterilized by baking at $200^{\circ} \mathrm{C}$ for $6 \mathrm{hrs}$. Cells were split onto $18 \mathrm{~mm}$ coverslips (for the Oli-neu cell line, coverslips were PLL coated) and were transfected at 70\% confluency. TransIT-LT1 (Mirus Biologicals) transfection reagent was used for the transfection. Briefly, $1 \mu \mathrm{g}$ of plasmid DNA was added together with $3 \mu \mathrm{L}$ of TransIT-LT1 to $100 \mu \mathrm{L}$ Opti-MEM solution (Gibco, Invitrogen). The mixture was incubated at RT for 24 min followed by gentle pipetting on top of the cells. $24 \mathrm{hrs}$ after transfections, the cells were either fixed or imaged live.

\subsubsection{Immunocytochemistry}

Primary cultures were prepared as described above. Cells were stained for endogenous and/or exogenously expressed proteins. For immunostaining, cells were washed once with PBS and fixed by the addition of $4 \%$ paraformaldehyde (Sigma-Aldrich) for $15 \mathrm{~min}$ at RT. Cells were then washed 3 times with PBS, permeabilized with 0.1\% TritonX-100 (in PBS) for 2 min and blocked with the blocking solution for 30 min. Blocking solution was then aspirated and cells were incubated with the primary antibodies, diluted in $10 \%$ blocking solution (blocking solution diluted appropriately in PBS) for $1 \mathrm{hr}$. Following primary antibody incubation, cells were washed with PBS three times and incubated further with appropriate secondary antibodies, also diluted in $10 \%$ blocking solution for $1 \mathrm{hr}$. Finally, the cells were washed with PBS several times and the coverslips were mounted in mowiol.

\subsubsection{Microscopy}

\section{Confocal Microscopy}

Confocal microscopy images of fixed cell samples were acquired with either Carl Zeiss LSM 510 microscope with a $63 \times$ oil -objective, or Leica TCS SP2 DMIRE2 microscope with a $63 \times$ oil plan-apochromat objective. For live imaging, cells were washed with PBS, incubated in Kreb-ringer's solution (Table 2.2) and images were 
acquired at $37^{\circ} \mathrm{C}$ with the Leica setup as described above. The temperature was controlled with an air flow chamber and cells were imaged up to $50 \mathrm{~min}$.

\section{Fluorescence recovery after photobleaching (FRAP)}

Primary oligodendrocytes or PtK2 cells were transfected for up to 24 hrs with the plasmids of interest as explained above. Cells were imaged live at $37^{\circ} \mathrm{C}$ with a confocal laser scanning microscope Leica TCS SP2 equipped with 63× NA 1.4 oil -objective using $512 \times 512$ pixel resolution $(1000 \mathrm{~Hz}$ scanning speed). The beam expander 1 was used in order to achieve efficient bleaching. Photobleaching was performed in five scans with the $488 / 514$ or $561 \mathrm{~nm}$ laser at $100 \%$ power within a $5 \mu \mathrm{m} \times 5 \mu \mathrm{m}$ rectangular region of interest, ROI (zoom-in mode). Pre- and postbleach fluorescence intensities (2 and 50 scans, respectively) were monitored with a laser power of $6 \%$ for $488 \mathrm{~nm}, 8 \%$ for $514 \mathrm{~nm}$ and $25 \%$ for $561 \mathrm{~nm}$ laser. 8-bit images were recorded every $0.657 \mathrm{sec}$. Processing and analysis of FRAP data was performed as described before (Axelrod et al., 1976). The fluorescence intensities for the post-bleach images were corrected for the background, as well as for bleaching followed by normalization with the average fluorescence intensity of pre-bleach images. Following equation was used for fitting the recovery curves:

$$
Y=Y o+a\left(1-e^{-b x}\right)
$$

Mobile fraction (also called as recovery\%) was then calculated from the obtained fit using the following equation:

$$
\text { Mobile fraction }=(Y o+a) * 100
$$

\section{Fluorescence decay after photoactivation (FDAP)}

Primary oligodendrocytes or PtK2 cells were transfected with Dendra2 fusion proteins for 24 hrs. Cells were imaged live at $37^{\circ} \mathrm{C}$ with a confocal laser scanning microscope Leica TCS SP2 equipped with $63 \times$ NA 1.4 oil-objective using $512 \times$ 512 pixel resolution (1000 Hz scanning speed). A $5 \mu \mathrm{m} \times 5 \mu \mathrm{m}$ ROI was excited with $80 \% 405 \mathrm{~nm}$ laser for 3-5 cycles in order to photoconvert Dendra2 from green to red. The decay of fluorescence intensities in the ROI was measured with time 
using 15\% $561 \mathrm{~nm}$ laser for $500 \mathrm{sec}$ with image acquisition every $10 \mathrm{sec}$. Processing and analysis of FDAP data was performed as described before (Serrels et al., 2009). Intensities in ROI were corrected for the background and normalized, with the first post-bleach intensity set to 1 . Same equation, as for the FRAP curves was used to fit the decay of the signal. Mobile fraction (decay\%) was calculated using the following equation:

$$
\text { Mobilefraction }=\left[1-\left(\frac{a}{a+y o}\right)\right] * 100
$$

\section{Fluorescence resonance energy transfer (FRET)}

PtK2 cells were cotransfected with GFP and mCherry fusion proteins for $24 \mathrm{hrs}$ as previously described (Fogel et al., 2011). Cells were then fixed with 4\% PFA followed by mounting in mowiol. For the experiment, FRET unit available at the Leica SP2 confocal setup equipped with $63 \times$ NA 1.4 oil-objective was used. Briefly, images were first acquired in both green (488 nm laser) and red (561 nm laser) channels prior to acceptor (red) photobleaching and were labelled as pre-bleached images. This was followed by bleaching of a $10 \mu \mathrm{m} \times 10 \mu \mathrm{m}$ region of interest (ROI) in the acceptor channel (4-8 bleaching cycles using $80 \% 561 \mathrm{~nm}$ laser power). Following acceptor bleaching, images were acquired in both channels and were labelled as post-bleach images. To calculate the FRET efficiency, increase in the fluorescence intensity in the green (donor) channel upon bleaching in the red (acceptor) channel was measured in the region of interest (Nawaz et al., 2009) and quantified using the formula:

$$
F \operatorname{RET}(\%)=\frac{F_{\text {acceptor }}(\text { postbleach })-F_{\text {acceptor }}(\text { prebleach })}{F_{\text {acceptor }}(\text { postbleach })}
$$

\section{Electron microscopy}

Optic nerves were fixed overnight with $4 \%$ paraformaldehyde and $0.2 \%$ glutaraldehyde. Samples were then proceeded as described before (Werner et al., 2007). Briefly, dissected optic nerves were infiltrated in 2.3 M sucrose in $0.1 \mathrm{M}$ phosphate buffer overnight, mounted onto aluminum pins for ultramicrotomy and frozen in 
liquid nitrogen. Ultrathin cryosections (Leica EM FC6) were picked up in a 1:1 mixture of $2 \%$ methylcellulose and $2.3 \mathrm{M}$ sucrose. Samples were immunolabelled with anti-GFP (1:100; Invitrogen, Darmstadt, Germany), anti-PLP (A431, 1:100) and anti-CNPase (1:250; Sigma-Aldrich, Munich) antibodies followed by gold-labelled secondary antibody staining (size of gold beads-10 nm, dilution-1:80, AURION, Wageningen, Netherlands). The sections were imaged with a Leo 912AB electron microscope equipped with a CCD camera $2048 \times 2048$ (Proscan, Scheuring, Germany).

\section{Atomic force microscopy (AFM)}

AFM indentation experiments were carried out with a MFP-3D (Asylum Research, Santa Barbara, CA, USA) as described before (Mueller et al., 1999). The spring constants of the silicon nitride cantilevers (OMCL-TR400PSA-3, Olympus, Japan) were individually calibrated by fitting the power spectrum to a simple harmonic oscillator using the Asylum research built in software routines. Proteins were resuspended in AFM buffer $\left(150 \mathrm{mM} \mathrm{NaCl}, 5 \mathrm{mM} \mathrm{KH_{2 }} \mathrm{PO}_{4}, \mathrm{pH} 7.4\right.$ titrated with $\mathrm{KOH})$ to a concentration of $0.25 \mathrm{mg} / \mathrm{mL}$. $40 \mu \mathrm{L}$ of solution was pipetted onto the Mica surface. AFM cantilever was then lowered on to the drop, and the system was incubated for $10 \mathrm{~min}$ to allow the proteins to bind. Afterwards, sample was rinsed 3 times with AFM buffer. Force vs. separation curves were recorded in liquid at RT with a constant speed of $1 \mu \mathrm{m} / \mathrm{s}$.

\subsubsection{Biomimetic SLB-MBP-GUV assay}

The following mole\% of lipid mixtures, mimicking inner leaflet myelin composition (Inouye and Kirschner, 1988), was used at a final concentration of $1 \mathrm{mg} / \mathrm{mL}$, cholesterol:PE:PIP2:PC:PS: SM (44\%:27\%:2\%:11.5\%:12.5\%:3\%). Lipids were dried in a speed-vac and then hydrated in $50 \mathrm{mM}$ HEPES containing $100 \mathrm{mM} \mathrm{NaCl}$ at $60^{\circ} \mathrm{C}$ for $1 \mathrm{hr}$ followed by sonication until a clear solution of small unilamellar vesicle (SUVs) was obtained. 2\% Hellmanex III detergent (Hellma analysis, Müllheim, Germany) was used for the cleaning of coverslips, followed by hydration via multiple washing steps in MilliQ water. SUVs were then spread onto the cleaned and hydrated 18 
mm glass coverslips. After washing the unbound lipids with $50 \mathrm{mM}$ HEPES, SLBs were incubated with either R3-GFP or MBP $(7 \mu \mathrm{M})$ for $40 \mathrm{~min}$ followed by washing and addition of giant unilamellar vesicles (GUVs) composed of PC:PS in 2:1 molar ratio. For the preparation of GUVs, electroformation method was used. It yields unilamellar vesicles with diameter ranging from 5 to $100 \mu \mathrm{m}$ (Kahya et al., 2005). The perfusion chamber used for vesicle preparation was equipped with two microscope slides, each coated with indium-tin oxide (ITO), which is electrically conductive and exhibits high light transmission in the visible range. GUVs were grown in the perfusion chamber at high temperature $\left(60^{\circ} \mathrm{C}\right)$ in presence of water, as a result of lipid swelling under an alternating current field (Kahya et al., 2005; García-Sáez et al., 2010).

\subsubsection{MBP aggregation assay}

Large unilamellar vesicles were prepared via extrusion protocol using mini-extruder (Avanti-Polar Lipids) according to the guidelines of the manufacturer. Briefly, lipids resembling inner leaflet composition: cholesterol:PE:PIP2:PC:PS:SM (44\%:27\%:2\%: $11.5 \%: 12.5 \%: 3 \%)$ were mixed together at a final concentration of $1 \mathrm{mg}$. The lipids were dried in speed-vac followed by addition of $1 \mathrm{~mL}$ HEPES buffer and brief sonication $\left(5 \mathrm{~min}, 30 \%\right.$ power and $\left.60^{\circ} \mathrm{C}\right)$ in a bath sonicator to obtain a milky hydrated lipid solution. This solution was then subjected to 5 freeze-thaw cycles (freezing in liquid nitrogen and quick thawing at $60^{\circ} \mathrm{C}$ ), followed by passing through miniextruder (20 times) using polycarbonate membranes with $100 \mathrm{~nm}$ pore size. The translucent solution thus obtained consisted of mostly $100 \mathrm{~nm}$ sized LUVs. Next, $50 \mu \mathrm{M}$ of either wild type or mutant MBP (each dissolved in $5 \mu \mathrm{L}$ of HEPES buffer) were mixed with $95 \mu \mathrm{L}$ of LUV solution and the mixture was further incubated at RT for $30 \mathrm{~min}$. The solutions were then subjected to ultracentrifugation at 100,000 g (Beckman TLA120.1). The pellet was resuspended in same volume as supernatant and the fractions were subjected to SDS-PAGE followed by Western blotting. 


\subsubsection{Image Analysis}

Table 2.6 provides a list of specific softwares used throughout the study for the purpose of primer designing, image processing, data quantification, plotting of data and statistical analysis. The software/applications marked with $\dagger$ are freely available online.

Table 2.6: List of softwares used in the study

\begin{tabular}{lll}
\hline Software & Application & Source/Manufacturer \\
\hline ApE $\dagger$ & DNA editing & by Wayne Davis, University of Utah \\
Image $\dagger \dagger$ & Image analysis & http://rsbweb.nih.gov/ij/ \\
MBF ImageJ $\dagger$ & Image analysis & http://wwwmacbiophotonics.ca/imagej/ \\
SigmaPlot 11 & Statistical analysis & Systat Software GmbH, Erkrath, Germany \\
\hline
\end{tabular}

\section{Correlation analysis}

To quantify the co-localization of exogenously expressed or endogenous proteins with MBP positive myelin membrane sheets, 8-bit confocal images with a pixel resolution of $1024 \times 1024$ were acquired in the green (expressed protein) and the red (MBP) channel. The signals in both channels were corrected for background followed by calculation of Pearson's correlation coefficient (McBiophotonics ImageJ, see Table 2.6) in the region of interest using the following equation:

$$
R_{x y}=\sum_{i=1}^{n} \frac{\left(x_{i}-\bar{x}\right)\left(y_{i}-\bar{y}\right)}{\sqrt{\sum_{i=1}^{n}\left(x_{i}-\bar{x}\right)^{2} \sum_{i=1}^{n}\left(y_{i}-\bar{y}\right)^{2}}}
$$

\section{Quantification of relative cell area}

In order to quantify the relative cell area (also termed \% cell area) occupied by the protein of interest, confocal images were acquired (Carl Zeiss LSM 510 microscope with a $63 \times$ oil -objective, or with Leica DMIRE2 microscope with a $63 \times$ oil planapochromat objective). Appropriate thresholds above the background were applied to all the images using the plugin: apply threshold from background in McBiophotonics ImageJ. Next, the percentage of area occupied by the signal above the 
threshold value was calculated in the region of interest (in this case one cell) using the measure tool.

\section{Profile plots}

In order to the see the overlap of signal intensities between the images in two channels, for example green and red, mean intensities were measured after background correction along a horizontal line $(100 \mu \mathrm{m}$ in size $)$ using measure tool in Image J, see Table 2.6.

\subsubsection{Statistical Analysis}

SigmaPlot 11.0 software (Systat Software Inc., San Jose, CA, USA) was used for all the statistical analysis. Unpaired $t$-test was used for comparing two groups in case of normal distribution, while for the samples not distributed normally MannWhitney Rank Sum Test was used. More than two groups were compared using one-way analysis of variance (ANOVA) in case of normal distribution. However, if the distribution was not normal, we used the nonparametric Kruskal-Wallis ANOVA on Ranks. 
"Research is to see what everybody else has seen and to think what nobody else has thought."

Albert Szent-Gyorgyi

\section{Results}

\subsection{MBP generates lipid-rich myelin membrane sheets}

\subsubsection{Cellular model of cultured oligodendrocytes}

To investigate the mechanisms involved in the generation of lipid-rich myelin membrane, we used cultured primary oligodendrocytes as the model system. OPCs were prepared from the brains of new born P0 mice. Cells were then shaken off from the astrocytic layer and plated in the differentiation medium. As these cultures differentiate and mature, they establish two major polarized domains: large, flat, two-dimensional compacted myelin membrane sheets and tubular, vein-like noncompacted myelin processes (Figure 3.1). The sheets resemble in vivo compact myelin (see unrolled myelin model Figure 3.1) in composition and are positive for MBP and PLP. The components of non-compact myelin like MAG and CNPase are restricted to the cellular processes.

After identifying the two major polarized domains in this cell culture model, we could address the question of how these domains are generated. One way to achieve polarized distribution of proteins is via differential sorting of cargo into distinct carrier vesicles. However, we found that exogenously expressed soluble proteins like GFP-Ub (Figure 3.2 A), GFP (Figure 3.2 B), thioredoxin (Figure 3.2 C) and ubiquitin (Figure 3.2 D) (Stoke's radii $3 \mathrm{~nm}, 2.5 \mathrm{~nm}, 2.0 \mathrm{~nm}$ and $1.9 \mathrm{~nm}$ respectively) were restricted to the processes. In addition, previous results from the lab had shown that the vesicular trafficking machinery is restricted to the processes 

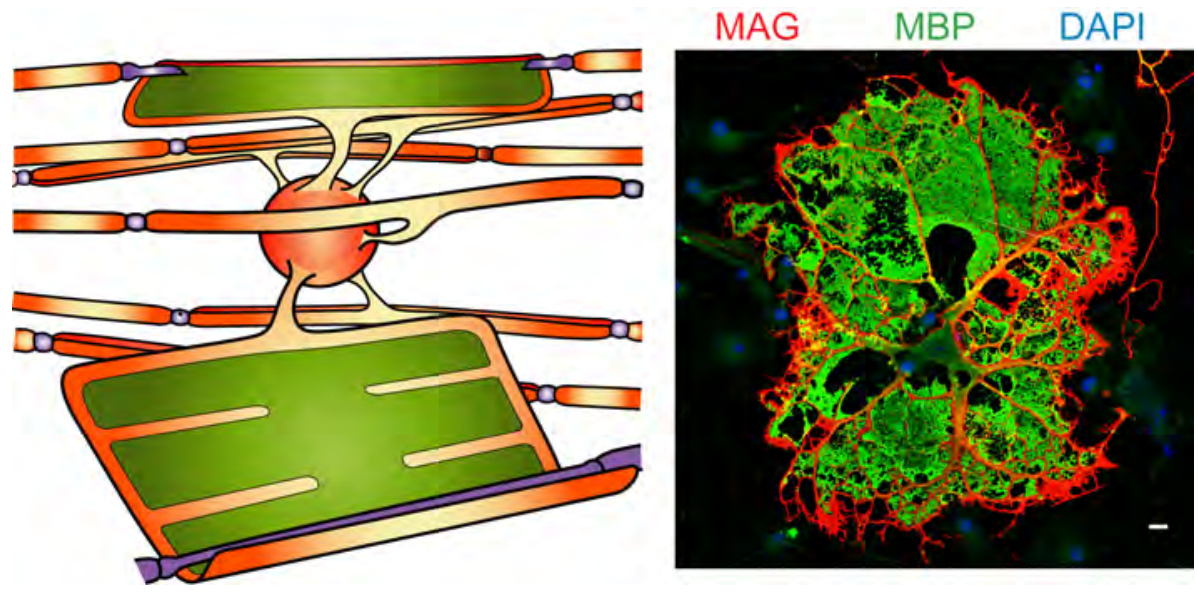

Figure 3.1: Model system of cultured oligodendrocytes to study myelin membrane biogenesis. These cultures intrinsically polarize their membrane into two major domains, the compact myelin (green in the left panel and MBP positive in the right panel) and the non-compact myelin (red in the left panel and MAG positive in the right panel).

of an oligodendrocyte (Dr. L. Yurlova), thereby excluding the polarized trafficking mechanism as a key for the generation of myelin membrane sheets.
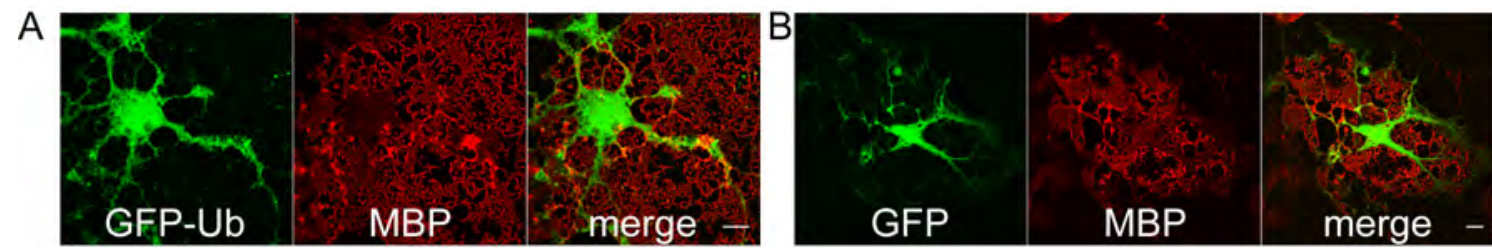

C

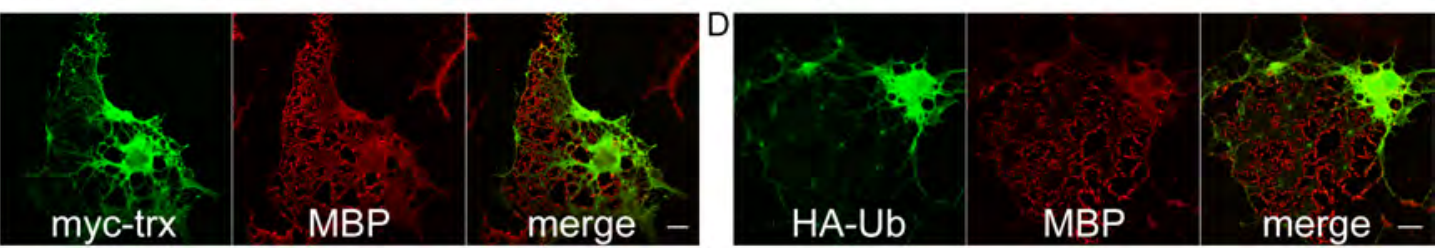

Figure 3.2: Localization of cytosolic proteins with decreasing Stoke's radii in oligodendrocytes. 4 DIV primary oligodendrocytes were transfected with (A) GFP-Ubiquitin (GFP-Ub), (B) GFP, (C) Myc-thioredoxin (Myc-trx) and (D) HA-Ubiquitin (HA-Ub) for 16 hrs followed by immunostaining. Myc-thioredoxin and HA-Ubiquitin were visualized by staining with myc and HA antibodies, respectively. Cells were co-stained for MBP to visualize the myelin membrane sheets. Scale bars represent $10 \mu \mathrm{m}$. All these cytosolic proteins are restricted from diffusing into MBP positive myelin-membrane sheets. 


\subsubsection{MBP regulates surface polarity in oligodendrocytes}

Next, we had a closer look at the secondary structure of transmembrane proteins within compact and non-compact myelin. PLP is a known compact myelin protein. It has 8 and 17 residues on the intracellular $\mathrm{N}$ - and C-terminus respectively. On the other hand, typical non-compact myelin proteins like MAG, Tmem10 (Opalin) and neurofascin-155 have 46, 90 and 110 residues respectively on the cytoplasmic sides. We tested an interesting hypothesis that proteins gain access into the myelin membrane sheets based on the size of their cytosolic domain. First, we designed a reporter construct where GFP was fused to the C-terminus of PLP (PLP-GFP). Next, we expressed this construct transiently in 4 DIV primary oligodendrocyte cultures for $16 \mathrm{hrs}$. Cells were then stained for the sheet/compact myelin marker MBP and scanned using a confocal setup (Leica SP2). Interestingly, PLP-GFP was excluded from the MBP positive sheets. It was mainly concentrated to the processes and the periphery of cells (Figure 3.3 A upper panel). Since PLP usually localizes to the compact myelin sheets, the above observation could be explained by the existence of a barrier that prevents entry of GFP-fusion proteins. Next, we studied the possibility whether a diffusion barrier exists within myelin membrane sheets. We explored the role of MBP, a peripheral myelin membrane protein, in this process. As explained in the introduction, MBP is one of the most abundant protein in myelin and its absence leads to severe myelination defects (Roach et al., 1983). Being an extremely basic protein, MBP interacts with the negatively charged lipids present in the inner leaflets of two opposing membranes, thereby bringing the two membranes in close apposition (Harauz et al., 2009). To investigate the role of MBP in the establishment of polarity, we transfected 4 DIV shiverer cells with the PLP-GFP construct for 16 hrs followed by immunostaining for MBP. We confirmed the absence of MBP with its negative staining in shiverer cultures (Figure $3.3 \mathrm{~A}$ lower panel). In contrast to the wild type cells, PLP-GFP was uniformly distributed in the membrane sheets of shiverer oligodendrocytes (Figure 3.3 A lower panel, see Figure 3.3 B for quantification). 


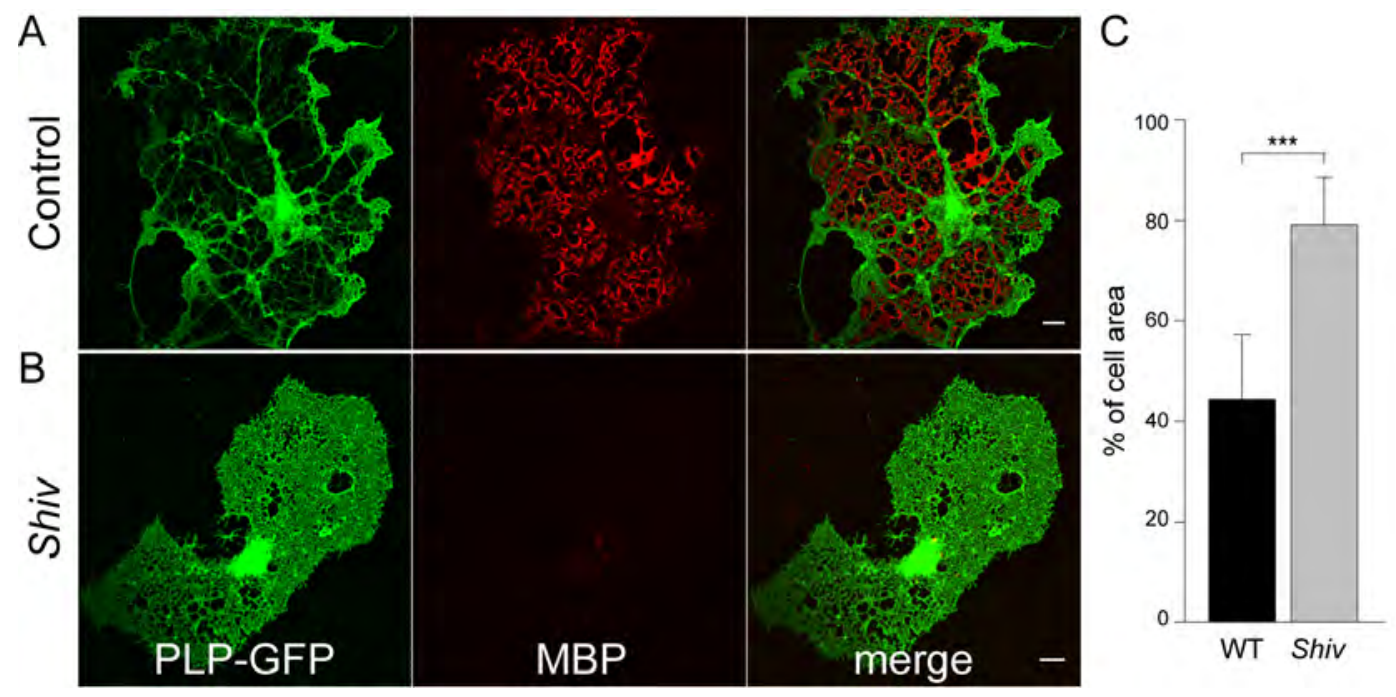

Figure 3.3: MBP establishes polarity in oligodendrocytes. (A) Localization of PLP-GFP (PLP tagged with GFP on the cytosolic side) in wild type (Control) or MBP deficient shiverer (Shiv) oligodendrocytes. 4 DIV cultures were transfected with PLP-GFP for 16 hrs followed by staining for MBP (Note the negative MBP staining for the Shiv cultures). Scale bars represent $10 \mu \mathrm{m}$. (B) Quantification of \% cell area occupied by PLP-GFP in wild type (WT) and shiverer (Shiv) cells. Bars show mean $\pm S D\left(n=20,{ }^{* * *} p<0.001, t\right.$-test). In comparison to WT cells, PLP-GFP is more uniformly distributed within the Shiv oligodendrocytes.

Further, we tested a number of different transmembrane proteins with GFP in their cytosolic domains (CD9-GFP, CD81-GFP, Tsp2-GFP, MOG-GFP). Each of these proteins localized to the processes in the wild type primary oligodendrocytes. However, all these proteins were uniformly distributed throughout the membrane sheets in shiverer oligodendrocytes (See the quantification of \% cell area in Figure 3.4 C). Also, endogenous proteins such as CNPase, MAG and septin 7 were present in the processes of wild type, but not shiverer cells (Figure 3.4 A, C).

The experiments above show the loss of cell surface polarity in cultures derived from MBP deficient shiverer mice. However, is MBP directly involved in polarity establishment? One possibility could be that another molecule forms the size barrier and the expression/localization of this molecule changes in the absence of MBP. To rule out the polarity loss as a secondary effect, we re-expressed MBP into the shiverer 

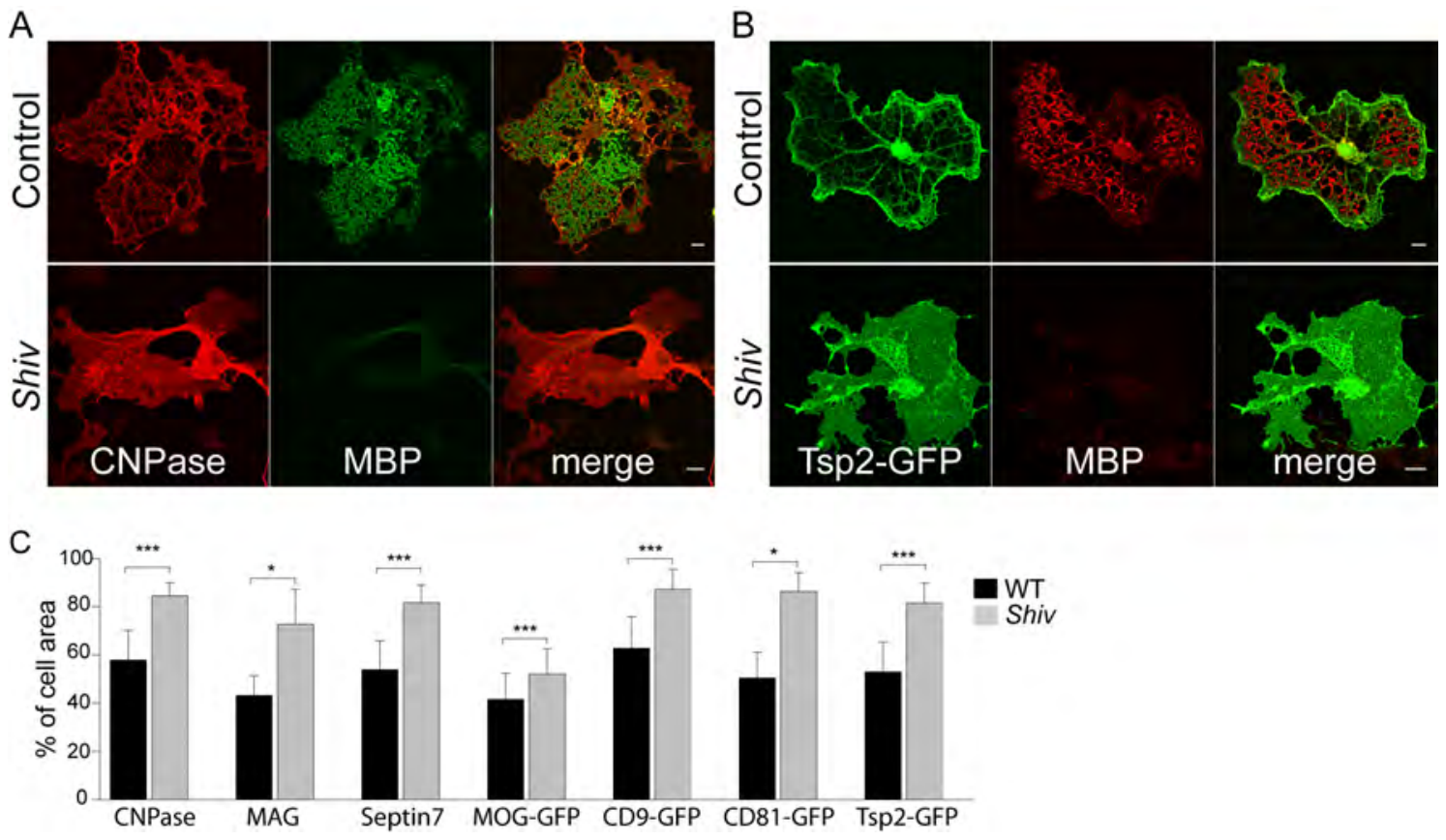

Figure 3.4: Localization of endogenous and exogenous proteins with bulky cytoplasmic domains in WT (Control) and shiverer (Shiv) cells. (A) Immunostaining of 5 DIV Control and Shiv cultures for CNPase. Negative MBP staining in the Shiv cells confirms its absence. Scale bars represent $10 \mu \mathrm{m}$. (B) Expression of Tetraspanin2-GFP (Tsp2-GFP) for 16 hrs into the control and Shiv cells followed by staining for MBP. Scale bars represent $10 \mu \mathrm{m}$. (C) Quantification of relative cell area occupied by endogenously expressed proteins: CNPase, MAG and Septin 7; and exogenously expressed proteins: MOG-GFP, CD9-GFP, CD81-GFP and Tsp2-GFP in wild type (WT) or shiverer (Shiv) cells. Note that GFP is on the cytosolic side for each of the exogenously expressed proteins. Bars show mean $\pm S D\left(n=20,{ }^{*} p<0.05,{ }^{* * *} p<0.001, t\right.$-test $)$. CNPase and MAG stainings were performed by Dr. L. Yurlova.

cells. MBP $14 \mathrm{kDa}$ with a C-terminal HA tag (MBP14-HA) was cloned into AAV2 viral expression vector. Shiverer cells were infected with the viral particles, 2-4 hrs after plating onto the coverslips. Cells were fixed at 6 DIV and CNPase distribution was investigated via immunostaining. Cells were also co-stained for the HA tag to visualize the expressed protein. Indeed, CNPase reallocated to the cellular processes upon expression of MBP14-HA (Figure 3.5). This experiment demonstrates that re-expression of MBP in shiverer cells is able to rescue cell surface polarity. 


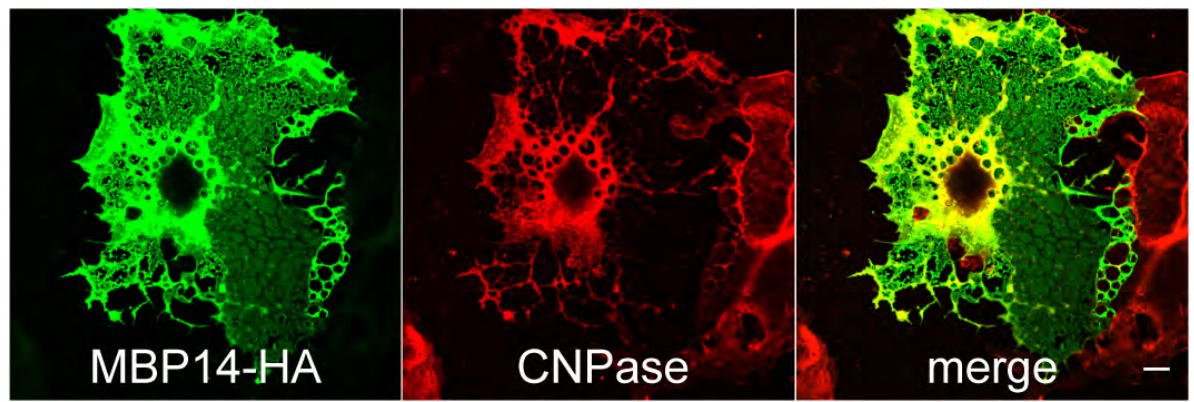

Figure 3.5: Restoration of polarity in shiverer oligodendrocytes. Shiverer cultures were infected with AAV2 viral particles expressing $14 \mathrm{kDa}$ isoform of MBP fused to an $\mathrm{HA}$ tag at the C-terminus (MBP 14-HA). Cells were immunostained for CNPase at 6 DIV. Expressed MBP was visualized by staining for the HA tag. Scale bar represents $10 \mu \mathrm{m}$. Expression of MBP redistributes CNPase to the processes, demonstrating the direct involvement of MBP in polarity establishment. Note that the virus was produced in collaboration with S. Kügler at the Department of Neurology, University Medicine, Göttingen.

So far, the data have shown that MBP regulates the distribution of membrane proteins. To analyze whether MBP also affects the distribution of cytosolic proteins, soluble GFP was expressed into wild type (WT) and shiverer (Shiv) oligodendrocytes. In both, WT as well as Shiv cells, GFP was excluded from the membrane sheets (Figure 3.6 A, see Figure 3.6 B for quantification). As an important control, we also expressed membrane anchored YFP, mem-YFP (anchored to the membrane via its N-terminal double palmitoylation signal sequence), which was targeted to the membrane sheets in Shiv, but not WT cells (Figure 3.6 C, see Figure 3.6 D for quantification). These experiments show that MBP-independent mechanisms regulate polarization of the cytosol in oligodendrocytes.

In another set of experiments, we fused GFP either to the extracellular (Figure 3.7 A upper panel) or to the cytoplasmic domain (Figure 3.7 A lower panel) of MOG, a protein that endogenously localizes to the membrane sheets in oligodendrocytes. MOG tagged with GFP on the extracellular domain co-localized with MBP in the myelin membrane sheets. On the other hand, MOG tagged with GFP on the cytosolic domain was restricted to the processes (see Figure 3.7 B for quantification). 

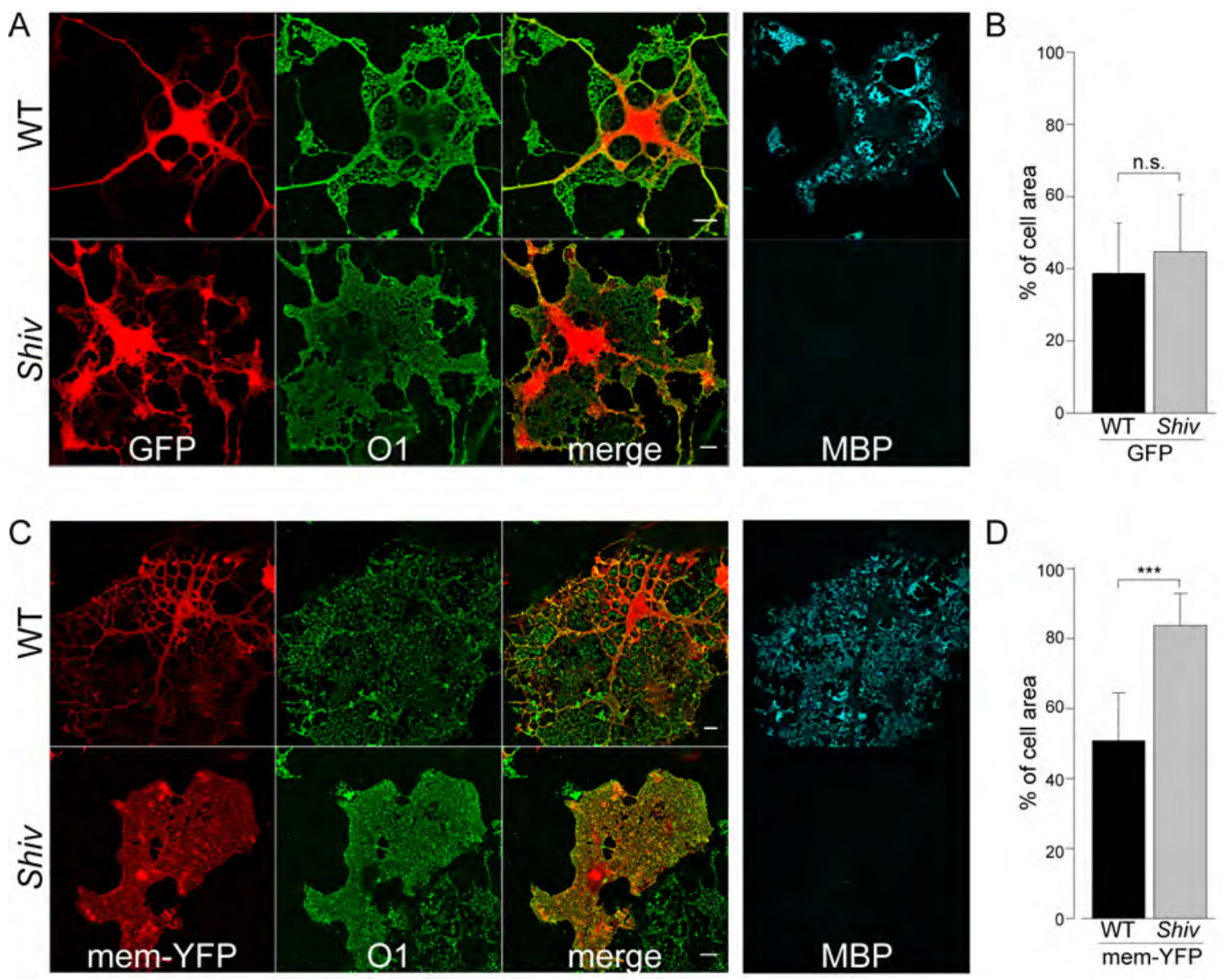

Figure 3.6: MBP regulates surface polarity in oligodendrocytes. (A) Oligodendrocytes were prepared from shiverer mice and the cells were immunostained for galactosylceramide (O1) to visualize the membrane sheets and for MBP to show its absence in shiverer cells. In MBP deficient oligodendrocytes, soluble GFP does not diffuse into the membrane sheets. Scale bars represent $10 \mu \mathrm{m}$. (B) Quantification of the relative cell area occupied by GFP in wild type (WT) and shiverer (Shiv) cells. Bars show mean $\pm S D\left(n=20,{ }^{* * *} p<0.001, t\right.$-test). (C) In MBP-deficient oligodendrocytes, membrane-anchored YFP (mem-YFP) is redistributed into the membrane sheets. Scale bars represent $10 \mu \mathrm{m}$. (D) Quantification of the relative cell area occupied by mem-YFP in wild type (WT) and shiverer (Shiv) cells. Bars show mean $\pm S D\left(n=20,{ }^{* * *} p<0.001, t\right.$-test). Note that the experiments with soluble GFP were performed by Dr. L.Yurlova. 
A

B
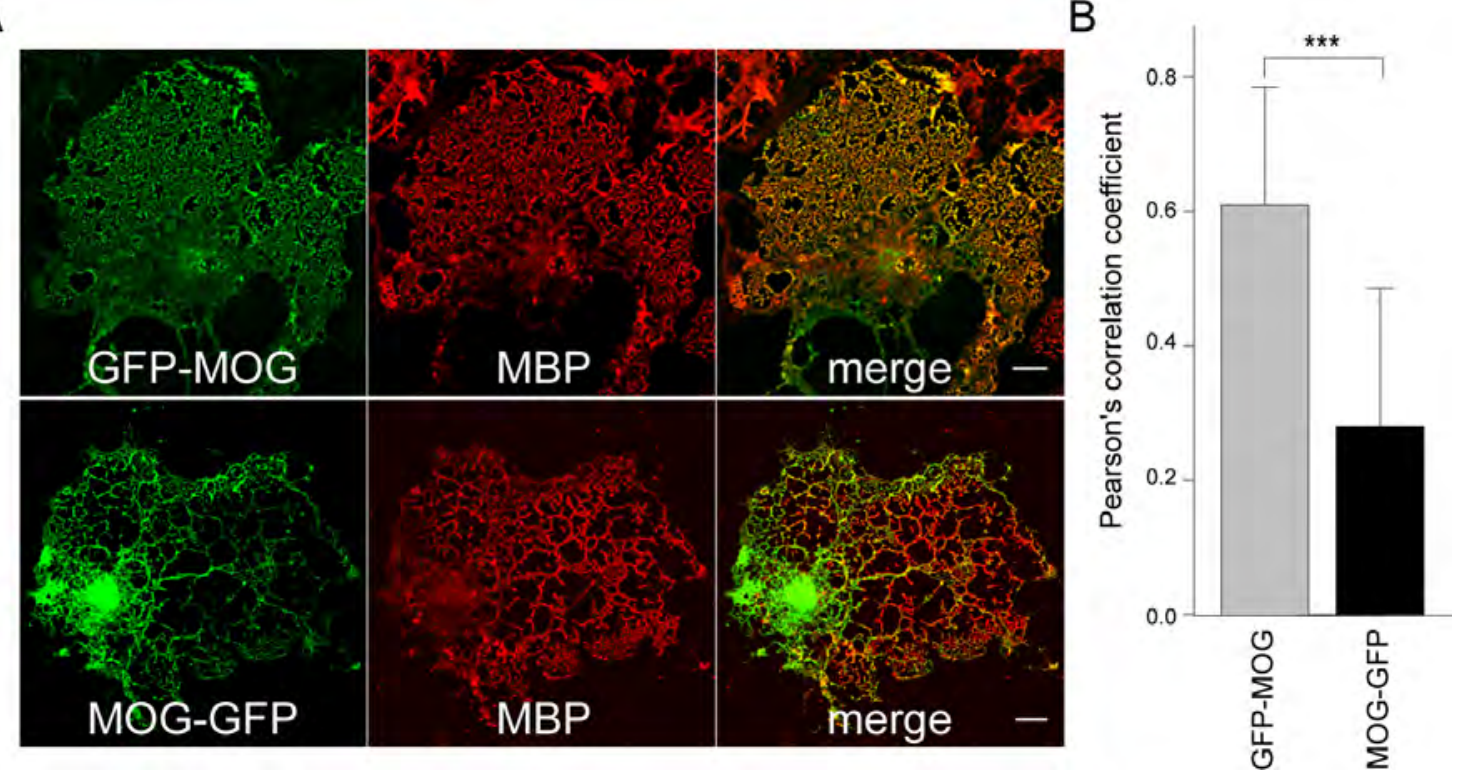

Figure 3.7: Fusion of GFP to the cytosolic, but not extracellular side restricts MOG to the processes. (A) Typical images of oligodendrocytes expressing GFP-MOG (extracellular GFP, upper panel) or MOG-GFP (cytosolic GFP, lower panel) and immunostained for MBP. Scale bars represent $10 \mu \mathrm{m}$. (B) Quantification of colocalization of expressed protein with MBP using Pearson's correlation coefficient shows that in comparison GFP-MOG, MOG-GFP is restricted from entering into the MBP positive myelin membrane sheets. Bars show mean $\pm S D\left(n=20,{ }^{* \star *} p<0.001\right.$, $t$-test).

\subsubsection{Cytoplasmic size-based diffusion of proteins into the myelin membrane sheets}

Thus, our data so far indicates that compact myelin proteins can be restricted from entering into the myelin membrane sheets by the exogenous addition of bulky cytosolic tags. The question now was whether non-compact myelin proteins would be able to gain access into the sheets upon truncation of their cytoplasmic parts. Therefore, in the next step, we designed variants of known non-compact myelin proteins with truncated cytoplasmic domains. We chose three known non-compact myelin proteins:MAG, Tmem10 and neurofascin-155, which have 46, 90 and 100 amino acid residues, respectively in the cytosolic domain (Tait et al., 2000; Charles et al., 2002; Golan et al., 2008; Yoshikawa et al., 2008; Kippert et al., 2008). The 
truncated variants of each of these proteins were expressed in 4 DIV primary oligodendrocytes and then localization into myelin membrane sheets was determined by co-staining for MBP (Figure 3.8 A, B and $\mathbf{C}$ right panels). As controls, full-length proteins were also expressed exogenously into the primary oligodendrocytes (Figure $3.8 \mathbf{A}, \mathbf{B}$ and $\mathbf{C}$ left panels). While the full-length proteins were detected mainly in the processes, the truncated variants for each of these proteins were uniformly distributed throughout the myelin membrane sheets (For quantification see Figure $3.9 \mathbf{B}, \mathbf{C}$ and $\mathbf{D})$.
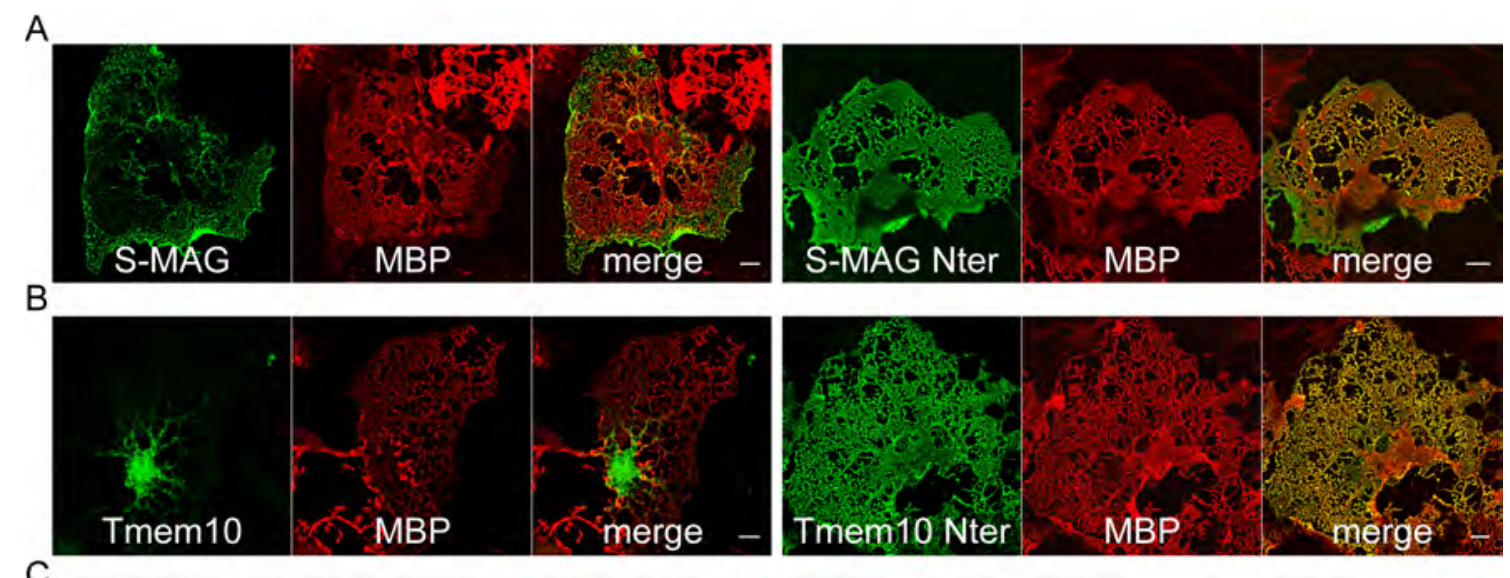

$\mathrm{C}$
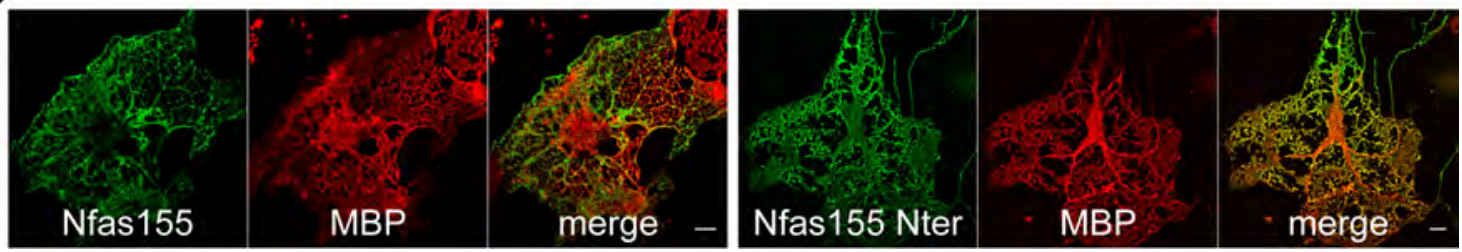

Figure 3.8: Non-compact myelin proteins lacking cytoplasmic domains redistribute into the myelin membrane sheets. Typical images of exogenously expressed (A) full length MAG (S-MAG) and truncated MAG (S-MAG Nter), (B) full length Tmem10 (Tmem10) and trucated Tmem10 (Tmem10 Nter) and (C) full length neurofascin-155 (Nfas155) and truncated neurofascin-155 (Nfas155 Nter). Expressed constructs were visualized by staining for the surface myc tags. Cells were co-stained for MBP in order to visualize the myelin membrane sheets. Scale bars represent $10 \mu \mathrm{m}$. Full length, but not truncated variants (containing only $\mathrm{N}$-terminal domains) are restricted from entering into the MBP positive myelin membrane sheets.

To define the exact size limit for the cytosolic domain, we designed serial truncation mutants of MAG, Tmem10 and neurofascin-155 (See the schematic, Figure 3.9 A). 
For each of these constructs, we found the cytosolic size limit to be less than 30 amino acids (aa) to enter into the compact myelin sheets. While constructs with either 10 or 20 aa on the cytosolic side colocalized with MBP in the myelin membrane sheets, the variants with 30 aa or more were restricted to the processes (See quantification, Figure 3.9 B, C and D). Localization of 30 aa long cytosolic domain containing MAG, Tmem10 and neurofascin-155 to the processes points towards size based sorting mechanism. However, could it be that there are retention signals within these 30 aa, which would restrict the localization to the processes/non-compact myelin domains? In order to test this possibility, we further designed probes where we added inert tags to the cytoplasmic domain of Tmem10 and checked for the localization into compact vs. non-compact myelin domains in primary oligodendrocyte cultures. As mentioned above, Tmem10 with 20 aa on the cytosolic domain is targeted to the MBP positive myelin membrane sheets. However, when we increased the size of this Tmem10 variant to 30 aa via the addition of an HA tag together with glycine linkers, the construct was restricted to the processes (Figure 3.9 E). This experiment demonstrates that sorting into the compact and non-compact myelin domains is independent of the amino acid sequence of the cytosolic domain.

Since MBP is a highly positively charged protein, one possibility for protein sorting into the myelin membrane sheets could be via electrostatic attraction or repulsion. To test this possibility, we designed variants of S-MAG and Tmem10 where the cytosolic domains were replaced with a stretch of either positive charged residues or negative charged residues. Next, we expressed these constructs in primary oligodendrocytes for 16 hrs followed by staining for MBP (Figure 3.10 A). Interestingly, all these proteins localized to the myelin membrane sheets regardless of the nature of the charge present on the cytosolic side (See Figure 3.10 B for quantification).

\subsubsection{Diffusion barrier characterization with FRAP}

The results so far have shown that MBP regulates polarity at the cell surface in oligodendrocytes leading to the formation of two major domains, compact and noncompact myelin. The presence of a diffusion barrier can also be identified by the lack of component exchange between the two sides of the boundary (Luedeke et al., 

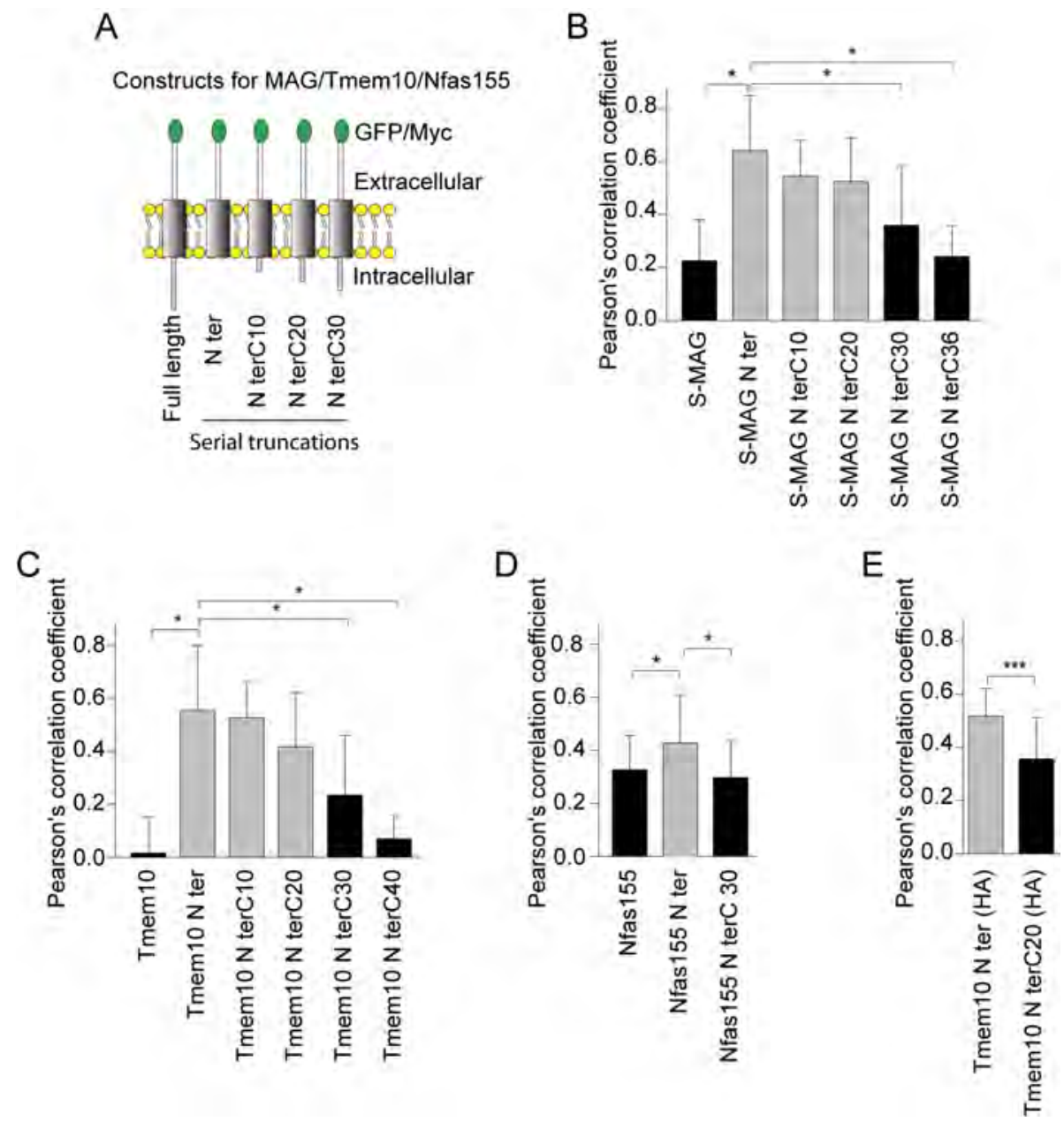

Figure 3.9: Cytoplasmic size limit for entering into the myelin membrane sheets is less than 30 aa. (A) Schematic representation of the serial truncation mutants of non-compact myelin proteins. Quantification of colocalization with MBP in case of the serial truncation mutants of (B) MAG, (C) Tmem10 and (D) Neurofascin-155. Bars show mean \pm SD $\left(n=20,{ }^{*} p<0.05\right.$, Kruskal-Wallis ANOVA on ranks). (E) Addition of inert HA tag to the cytosolic domain of Tmem10 N terC20 resulted in decreased colocalization with MBP. As a positive control HA tag was added to the cytosolic end of Tmem10 N ter. Bars show mean $\pm \operatorname{SD}\left(n=20,{ }^{* *} p<0.001, t\right.$-test $)$. 

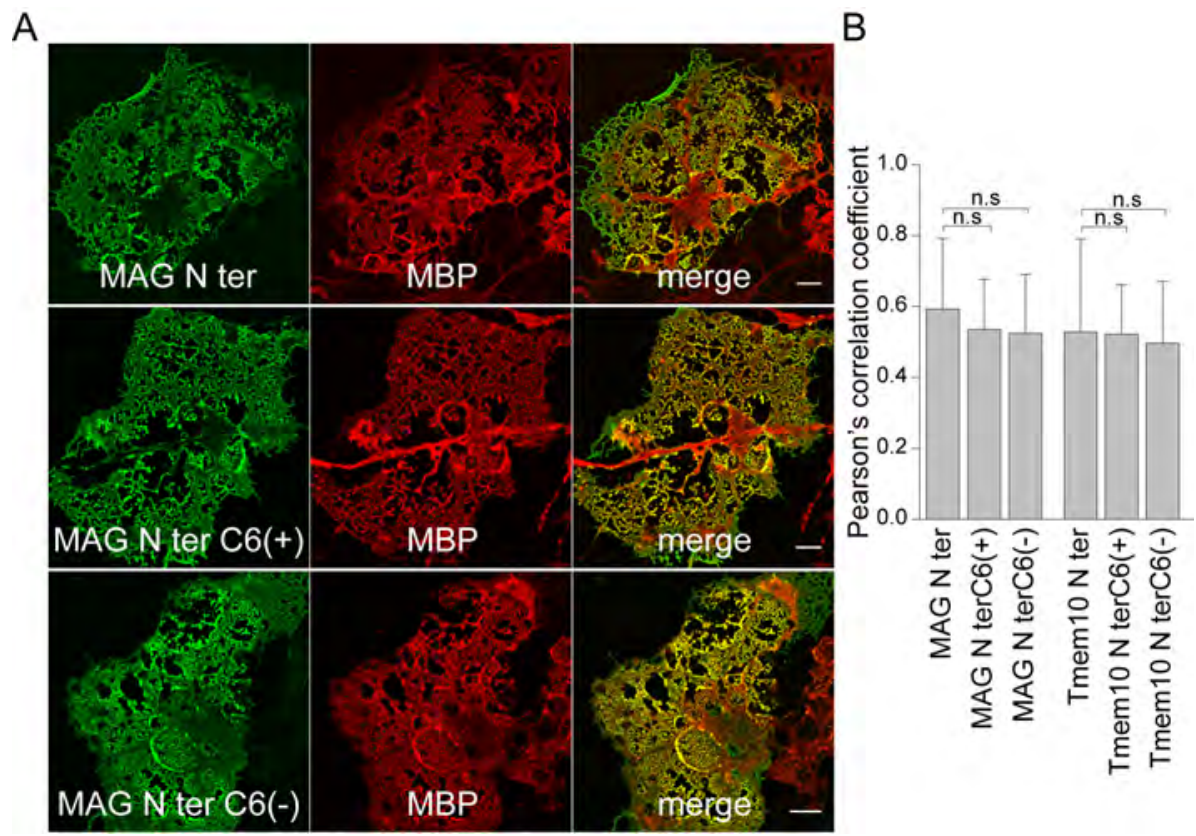

Figure 3.10: Charge of the cytosolic domain does not influence diffusion into the myelin membrane sheets. Cytosolic domains of MAG and Tmem10 were truncated and replaced by four glycine residues followed by either 6 positive or 6 negative charged residues. (A) Typical images of the charged variants of MAG that were visualized by staining for the extracellular myc tags. Myelin membrane sheets were visualized by co-immunostaining for MBP. Scale bars represent 10 $\mu \mathrm{m}$. (B) Quantification showing colocalization of the indicated proteins with MBP. Bars show mean $\pm S D(n=40, p>0.05$, ANOVA, n.s. indicates no significant difference). Charge did not influence the distribution of MAG or Tmem10 into the myelin-membrane sheets. Note that all charged forms of MAG and Tmem10 have only 10 amino acids within the cytosolic domain.

2005). Since MBP itself is a part of the developing compacted myelin sheets, we chose areas within the sheets where non-compact myelin components like MAG was still around. 4 DIV primary oligodendrocytes were surface stained with primary antibodies against MAG followed by Fab fragments of secondary antibodies. Next, we bleached these areas within the sheets and measured signal recovery with time (Figure 3.11 A upper panel). MAG was virtually immobile within the sheets as shown by the recovery curve (Figure 3.11 B). Further, we measured the mobility of MAG molecules in shiverer cells (Figure 3.11 A lower panel). As expected, the mobility was drastically increased in comparison to the wild type cells (see Figure $3.11 \mathbf{B}$ for the recovery curves). In a complementary approach, we looked at the 
mobility of truncated Tmem10 lacking the cytosolic domain. First, the construct was transfected into 4 DIV primary oligodendrocytes for $16 \mathrm{hrs}$. Transfected cells were identified by staining for the surface myc tags (first with primary antibodies against myc tag followed by labeling with Fab fragments of secondary antibodies). As a control, we also transfected full length Tmem10 (90 aa in the cytoplasmic domain) into the cells. Again, mobility of truncated Tmem10 was dramatically higher within the sheets in comparison to the full length construct (Figure 3.11 C).

\subsubsection{MBP regulates lipid/protein ratios in myelin}

The filtering mechanism elucidated above could probably explain why compact myelin is lipid-rich and contains only a restricted set of proteins. To analyze whether MBP indeed regulates the ratio of proteins to lipids in myelin membrane, we subjected brains from wild type (WT) and shiverer (Shiv) mice to a myelin purification protocol (Larocca and Norton, 2007). Next, we measured the protein and lipid content (cholesterol and fatty acids) of these fractions. Shiverer myelin had a much higher ratios of protein-to-cholesterol (Figure 3.12 A) and protein-to-fatty acids (Figure 3.12 B) in comparison to the WT myelin.

Because purified myelin membrane fractions from shiverer mice might be contaminated with membranes from other sources, we analyzed the protein abundance in the membrane sheets of shiverer (Shiv) and wild type (WT) oligodendrocytes in culture. The cell surface of oligodendrocytes was stained with fluorophore-conjugated concanavalin A (ConA) or wheat germ agglutinin (WGA) to label the terminal $\alpha$-D-mannosyl, $\alpha$-D-glucosyl residues, sialic acid, or N-acetyl-glucosamine carbohydrate groups that are present on many glycoproteins (Figure 3.13 A and $\mathbf{C}$ upper panels). Membrane sheets from shiverer oligodendrocytes were labeled more extensively by the fluorophore-conjugated ConA and WGA in comparison to the sheets from the wild type cells (Figure 3.13 A and C lower panels, see Figure 3.13 B and $\mathbf{D}$ for quantification). Furthermore, in another set of experiments, surface cysteine residues were reduced with tris(2-carboxyethyl)phosphine (TCEP) and then fluorescent maleimide was used to probe for the cysteine residues. These exper- 


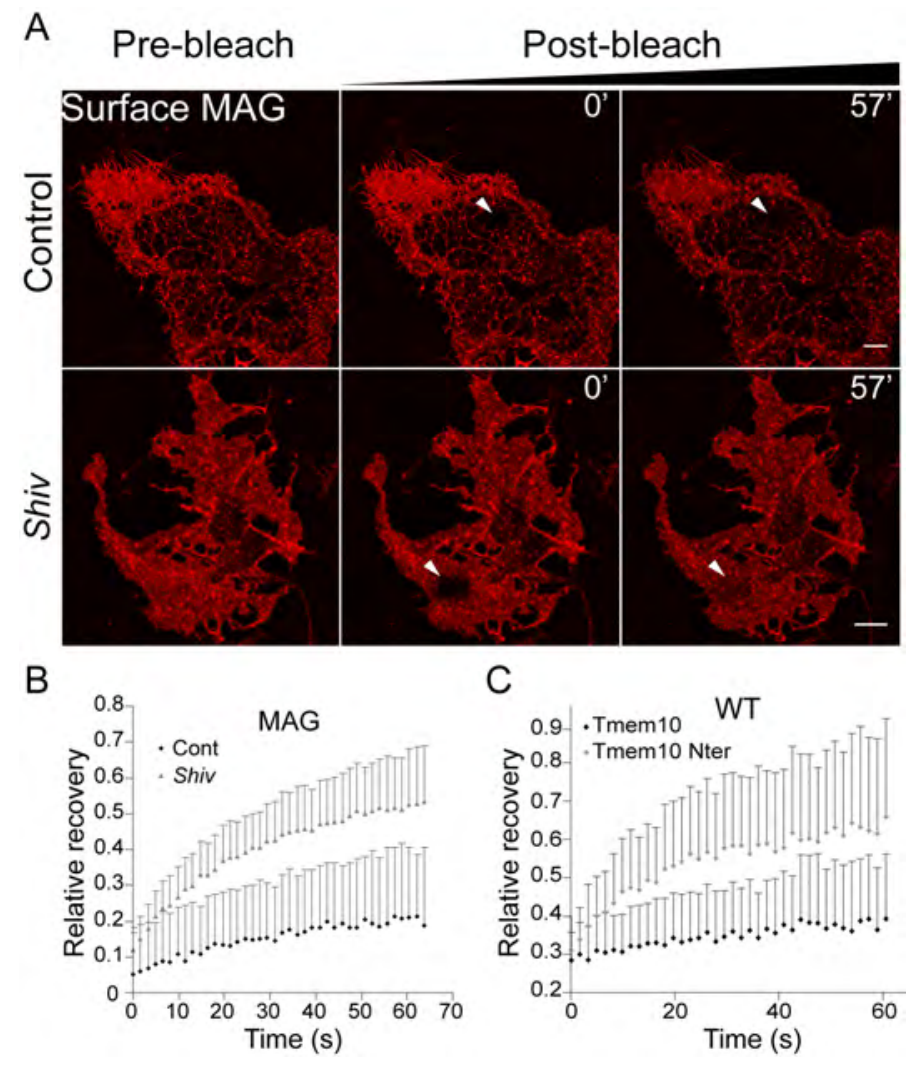

Figure 3.11: FRAP experiments with compact and non-compact myelin proteins. (A) 4 DIV wildtype (Control) or shiverer (Shiv) cells were surface labeled against MAG using anti-MAG primary antibodies $\left(15 \mathrm{~min}, 4^{\circ} \mathrm{C}\right.$ ) followed by Rhodamine conjugated Fab fragments of the secondary antibodies $\left(15 \mathrm{~min}, 4^{\circ} \mathrm{C}\right)$. Cells were then imaged live at $37^{\circ} \mathrm{C}$ for up to $45 \mathrm{~min}$. Typical prebleach and post-bleach images (at time points $0 \mathrm{sec}$ and $57 \mathrm{sec}$ ) are shown for the Control (upper panel) and Shiv cells. Region of interests are marked with arrow heads. Scale bars represent 10 $\mu \mathrm{m}$. (B) FRAP was measured by bleaching a squared ROI $(5 \mu \mathrm{m} \times 5 \mu \mathrm{m})$ within the membrane sheets (Control or Shiv) and fluorescence recovery was measured with time. Recovery curves are presented as graphs ( $n=20$ cells). (C) 4 DIV oligodendrocytes were transfected with full length Tmem10 and truncated Tmem10 lacking the cytosolic domain (Tmem10 Nter) for 16 hrs. Cells were surface stained for the extracellular myc tags, first with monoclonal primary antibodies (15 min, $4^{\circ} \mathrm{C}$ ) and then with Rhodamine coupled Fab fragments of anti-mouse secondary antibodies $\left(15 \mathrm{~min}, 4^{\circ} \mathrm{C}\right)$. The recovery curves are presented as graphs ( $\mathrm{n}=20$ cells).

iments revealed that membrane sheets from shiverer oligodendrocytes contain a much higher intensity of fluorescent label than the wild-type membranes, showing that the protein concentration was elevated in the shiverer sheets (Figure $3.13 \mathbf{E}$, 

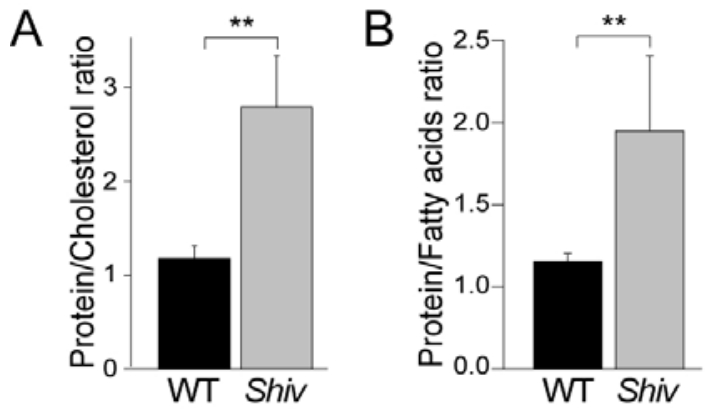

Figure 3.12: Lipid-to-protein ratios are altered in the shiverer myelin. Protein and lipid amounts were estimated in myelin purified from 4-7 week old wild type (WT) and shiverer (Shiv) mice. (A) Quantification of Protein/Cholesterol and (B) Protein/Fatty acid ratios in WT and Shiv myelin reveals that in the absence of MBP, protein-to-lipid ratios increase significantly. Bars show mean $\pm \mathrm{SD}\left(\mathrm{n}=6\right.$ animals, ${ }^{* *} \mathrm{p}<0.05, t$-test). Note that lipid amounts in the myelin samples were estimated in collaboration with C. Goebel at the Department of Plant Biochemistry, University of Göttingen.

for quantification see Figure $3.13 \mathbf{F}$ ). Together, the results so far indicate that a simple physical mechanism regulates protein-to-lipid ratios in myelin.

\subsubsection{In vivo validation of size barrier hypothesis}

Using the model system of cultured oligodendrocytes, we have shown that oligodendrocytes employ MBP as a physical filter, which restricts the diffusion of proteins with bulky cytoplasmic domains into the myelin membrane sheets. We next aimed to test the relevance of size barrier mechanism in vivo. We chose a transgenic mice line expressing membrane anchored GFP. In these animals, GFP is anchored to the membrane via double palmitoylation 13 aa sequence, derived from the N-terminal region of PLP (Spassky et al., 2001). This mice line will be referred to as 13aaPLPGFP from here onwards. Optic nerves from 13aaPLP-GFP mice were dissected and processed for cryo-electron microscopy (cryo-EM). It has been shown in previous studies that 13aaPLP-GFP in these mice is incorporated into the myelin membrane (Wight et al., 1993). However, precise localization of this protein within myelin domains was not investigated before. We immunolabeled cross-sections of optic nerves with GFP antibodies followed by gold-conjugated secondary antibody labeling. In- 

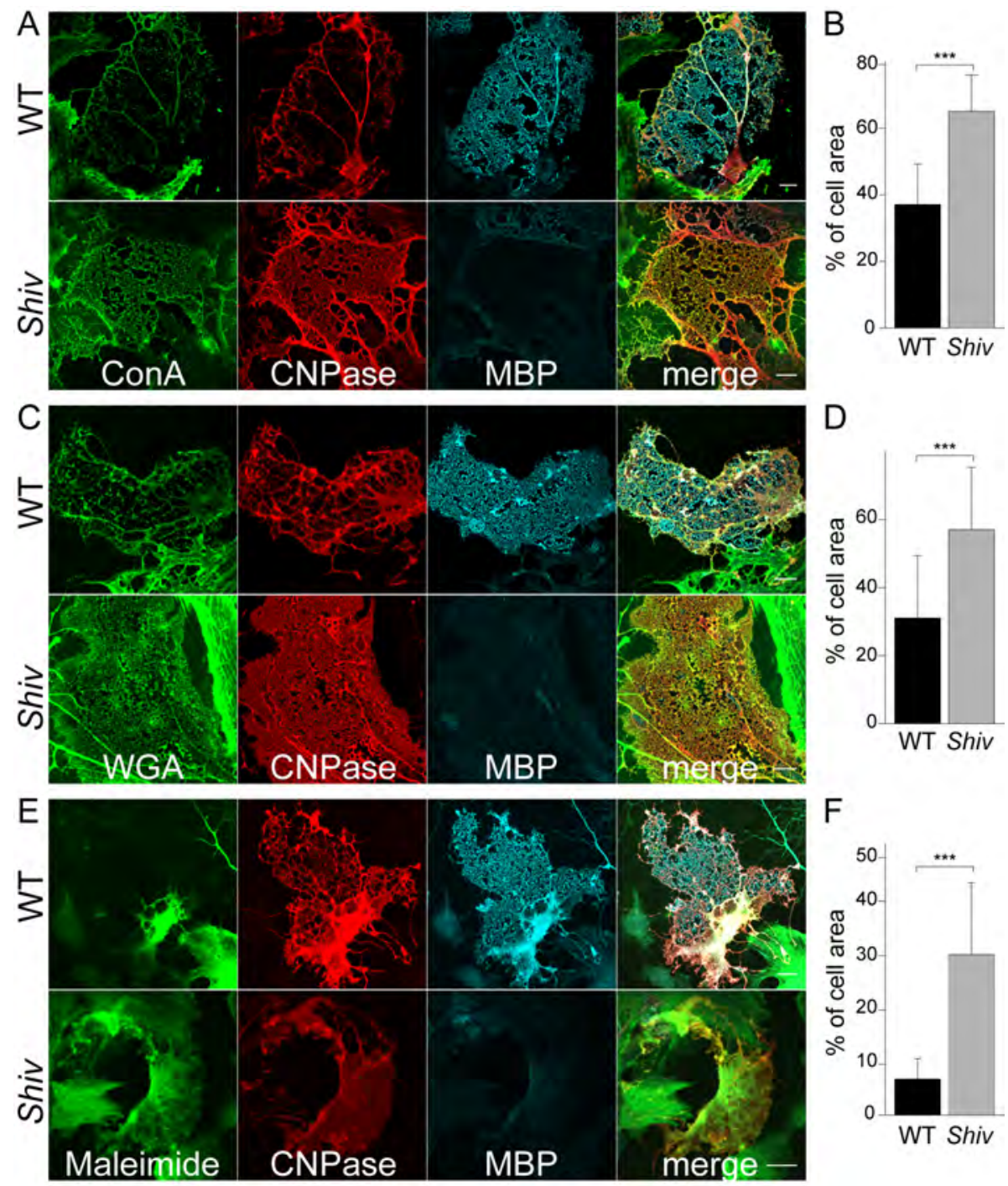

Figure 3.13: Cell surface abundance of proteins in WT vs. Shiv membrane sheets. Cell surface glycoproteins in oligodendrocytes were visualized by staining with fluorophore-conjugated lectins, (A) ConA and (C) WGA. CNPase was used as a marker for oligodendrocytes (negative MBP staining in Shiv cells is shown for confirmation). Scale bars represent $10 \mu \mathrm{m}$. Quantification of relative cell area occupied by (B) ConA and (D) WGA in WT and Shiv cells shows more uniform distribution of both lectins in the absence of MBP. Bars show mean $\pm S D\left(n=20,{ }^{* * *} p<0.001\right.$, $t$-test). (E) Cell surface disulfide bonds were reduced with TCEP and then fluorescent maleimide (coupled to Alexa-488) was used to label the cysteine residues of surface proteins through their free $\mathrm{SH}$ groups. CNPase was used as a marker for oligodendrocytes (negative MBP staining in shiverer cells is shown for confirmation). Scale bars represent $10 \mu \mathrm{m}$. (F) Quantification showing mean pixel intensity in arbitrary units (AU) of Alexa-488-conjugated maleimide in regions of interest in the membrane sheets of WT and Shiv cells. Fluorescence intensity is significantly higher in the membrane sheets of Shiv cells indicating an increment in surface proteins. Bars show mean $\pm S D\left(n=40,{ }^{* * *} p<0.001\right.$, Mann-Whitney Rank Sum test). 
terestingly, GFP signal was specifically enriched in the periaxonal region of myelin and was almost completely excluded from the compact myelin (Figure $3.14 \mathrm{~A}$ left panel). To confirm the specificity of the GFP antibody, we also immunolabeled the optic nerves from the wild type mice. Indeed, background was found to be negligible when compared to the signal observed in 13aaPLP-GFP transgenic mice (data not shown). As an important positive control, we also looked at the distribution of endogenous PLP in these transgenic mice (Figure 3.14 A right panel). As expected, PLP signal was specifically enriched in the compact myelin areas (80\% of the total labeling, see Figure 3.14 B for quantification). This experiment nicely illutrates the exclusion of a bulky protein from the compacted myelin sheaths in vivo.
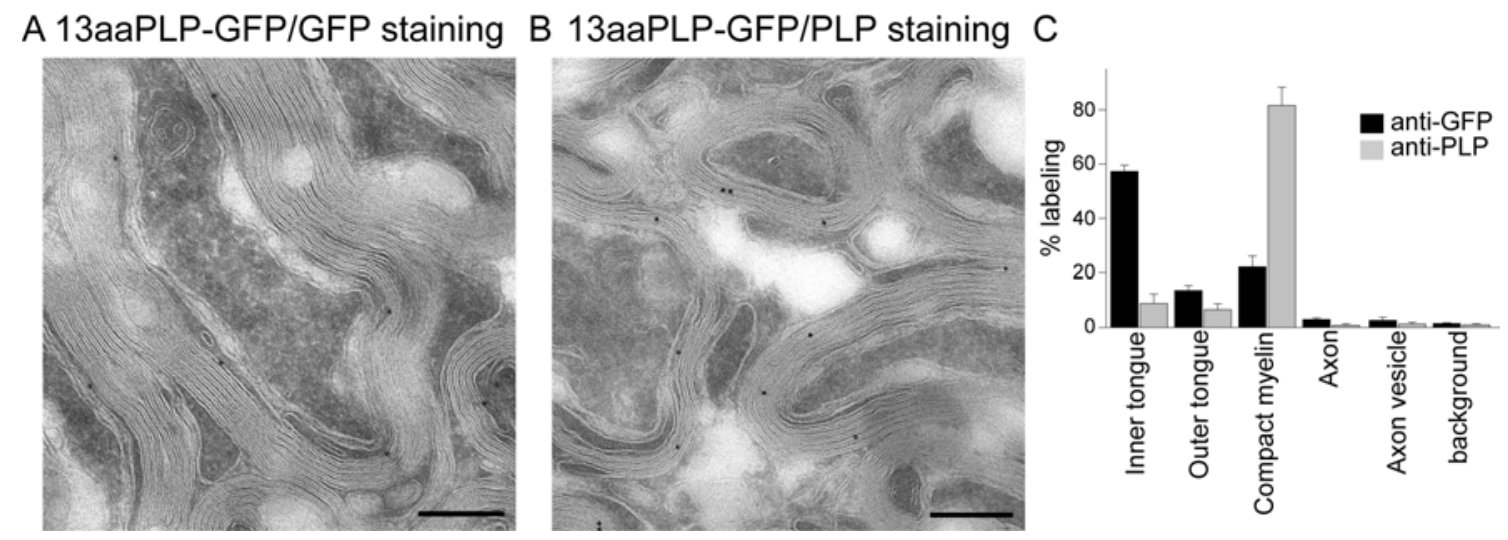

Figure 3.14: In vivo localization of membrane-anchored GFP in myelin. Immunoelectron microscopic analysis of optic nerves from 6-month old transgenic mice expressing 13aaPLP-GFP. (A) GFP antibodies were used to visualize 13aaPLP-GFP. Scale bar represents $200 \mathrm{~nm}$. (B) Anti-PLP antibodies were used to detect endogenous PLP. Scale bar represents $200 \mathrm{~nm}$. (C) Quantification for the $\%$ of total labeling revealed enrichment of 13aaPLP-GFP in the inner tongue of myelin. Bars show mean $\pm S D$ ( $n=3$ animals). In a sharp contrast, endogenous PLP is mainly concentrated in the compact myelin areas. Note that these experiments were performed in collaboration with N. Snaidero at Max Planck Institute of Experimental Medicine, Göttingen.

Next, we compared the in vivo distribution of the non-compact myelin protein, CNPase between wild type (WT) and shiverer mice. Optic nerves from 3-week old animals were dissected and processed for cryo-EM. Samples were immunolabeled for CNPase. While the localization of CNPase was mainly restricted to the innermost 


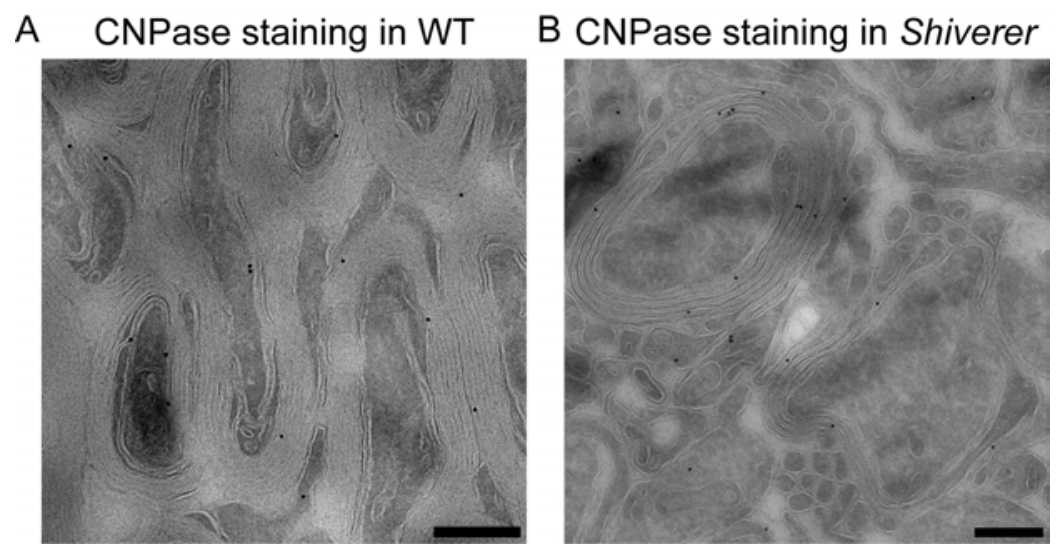

Figure 3.15: Comparison of CNPase distribution within myelin between WT and Shiv mice. Immunoelectron microscopy of optic nerves dissected from 21-day old (A) wildtype (WT) and (B) shiverer (Shiverer) mice. Staining with antibodies reveals that CNPase is mainly found in the inner most tongue of the myelin sheath in wild-type mice, but not shiverer mice. Representative pictures of 30 analyzed images are shown. Scale bars represent $200 \mathrm{~nm}$. Note that these experiments were performed in collaboration with $\mathrm{N}$. Snaidero at Max Planck Institute of Experimental Medicine, Göttingen.

tongue of myelin in the wild type animals (Figure $3.15 \mathbf{A}$ ), the distribution was rather uniform, throughout the myelin sheaths in the shiverer mice (Figure 3.15 B). These experiments clearly demonstrate the in vivo relevance of the size barrier mechanism.

\subsubsection{Estimation of MBP amounts in primary oligodendro- cytes}

For the size barrier model to be plausible, MBP must be expressed at high enough levels to coat the entire surface of membrane sheets. To test this, we first measured the average surface area of 7 DIV primary oligodendrocytes. The average area was around $7,511 \pm 2,146 \mu \mathrm{m}^{2}$ (mean $\pm \mathrm{SD}$ and $\mathrm{n}=50$ cells). For estimating MBP amounts, we subjected cell lysate from 7 DIV oligodendrocytes to SDS-PAGE analysis followed by Coomassie staining (Figure 3.16). Purified MBP was used to prepare the protein standards for calibration. From the standard curve, we in- 


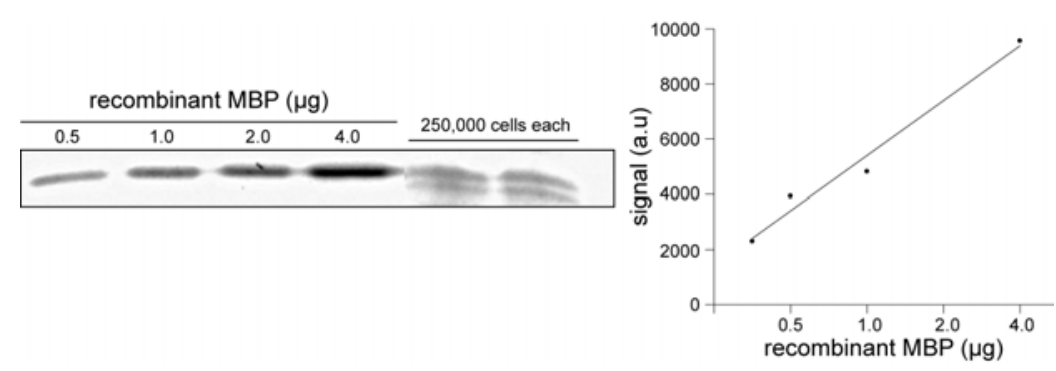

Figure 3.16: Estimation of MBP amounts in primary oligodendrocytes. 7 DIV oligodendrocyte lysates were subjected to SDS-PAGE analysis to estimate the amount of MBP per cell. Purified MBP was used for preparing the standards. We estimated 466,195,516 $\pm 84,028,306$ (mean \pm SD) MBP molecules per oligodendrocyte ( $n=4$ independent experiments).

trapolated amounts of MBP per cell followed by the calculation for the number of molecules. Our estimates indicated 466,195,516 $\pm 84,028,306$ (mean \pm SD) MBP molecules per oligodendrocyte ( $\mathrm{n}=4$ independent experiments). These MBP molecules should cover an area of $7,915 \pm 661 \mu \mathrm{m}^{2}$, if one takes the dimension of MBP as $7 \mathrm{~nm} \times 2 \mathrm{~nm}$ (Beniac et al., 1997; Ridsdale et al., 1997). Thus, there would be sufficient number of MBP molecules in a cell to cover the entire surface.

\subsubsection{A biomimetic assay and barrier reconstitution}

To further reconstitute the barrier properties of MBP outside the cellular environment, we went on to develop a minimal component biomimetic membrane system, based on supported lipid bilayers (SLBs). In the first step, we purified MBP $14 \mathrm{kDa}$ (from here on referred as MBP) in large amounts using bacterial expression system. Second, we prepared small unilamellar vesicles (SUVs) from artificial lipids with 44 mol\% cholesterol, $27 \mathrm{~mol} \%$ PE, $2 \mathrm{~mol} \%$ PIP2, $11.5 \mathrm{~mol} \%$ PC, $12.5 \mathrm{~mol} \%$ PS and $3 \mathrm{~mol} \% \mathrm{SM}$. The mol\% for each lipid corresponds to the values reported in literature for the inner leaflet of myelin (Inouye and Kirschner, 1988). In addition, SUVs contained $0.1 \%$ of 1,2-dihexadecanoyl-syn-glycero-3-phosphoethanolamine (DHPE)Texas Red for visualization with light microscopy later (see below). SUVs were added to detergent-treated and pre-cleaned coverslips fitted into the imaging chambers. The tension exerted by the hydrated coverslips causes spreading of the SUVs leading to the formation of SLB (Richter et al., 2006). Next, we incubated SLBs with 
$7 \mu \mathrm{M}$ MBP for 30 min followed by extensive washing. Finally, we added giant unilamellar vesicles (GUVs) with $66 \mathrm{~mol} \%$ PC, $33 \mathrm{~mol} \%$ PS and $1 \mathrm{~mol} \%$ DHPE-Texas Red (for visualization) on top of SLBs to sandwich MBP between two membranes. Strikingly, when GUVs were added on top of the SLB, MBP induced deformation of GUVs, which was immediately followed by their spreading onto the SLB (Figure 3.17 B right panel). No GUV spreading was observed for the control sample where MBP was omitted (Figure $3.17 \mathbf{C}$ right panel). We also purified a membrane anchoring GFP probe, R3-GFP. This protein binds to the negatively charged lipids via the N-terminal R3 basic stretch (For the schematic see Figure 3.17 A). Next, we added $7 \mu \mathrm{M}$ of R3-GFP to the SLB-MBP-GUV sandwich. As expected, MBP together with the SLB and the GUVs formed a tight barrier for the diffusion of membrane-anchored GFP (Figure 3.17 B left panel). As a control experiment, we left out MBP and in this condition R3-GFP was uniformly distributed throughout the SLB (Figure 3.17 C left panel).

We also performed the SLB-MBP-GUV assay by adding the components in a different order. First, we added R3-GFP to the SLBs followed by the addition of MBP. Afterwards, we washed off the unbound R3-GFP. At this point, R3-GFP was uniformly distributed on the SLBs. Finally, GUVs were added from the top to complete the sandwich. As expected, in the presence of MBP, GUVs attached to the SLB followed by spreading (in the control sample MBP was skipped, Figure 3.18 B). Surprisingly, at these attachment sites, R3-GFP signal decreased hinting towards extrusion of bulky R3-GFP (Figure 3.18 A). Another possibility is that the GFP fluorescence decreases due to quenching by the red fluorophore of GUVs. However, Figure 3.18 C clearly shows that GFP signal decreases from certain areas on the SLBs even when we used unlabelled GUVs.

These in vitro experiments clearly demonstrated self-sufficiency of MBP in generating molecular sieve with minimal components. Importantly, the experiments also highlighted the importance of sandwiching MBP between two opposing membranes for the efficient barrier formation. But the question that comes next is whether any protein that cross-links the two membranes would deform the GUVs and thus form the molecular sieve. To test this possibility, we used pentavalent O4 IgM antibodies 


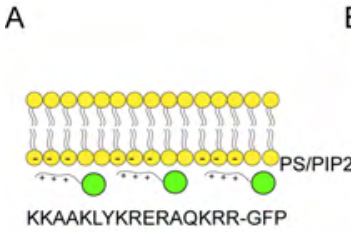

R3-GFP
B

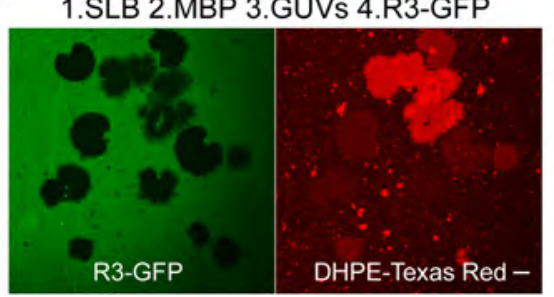

C

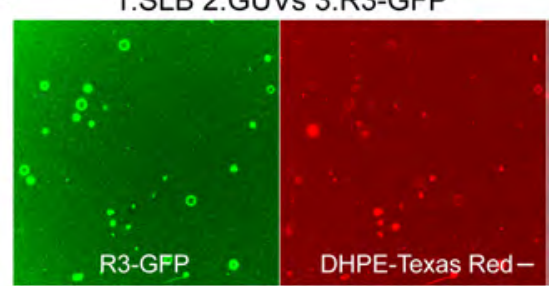

Figure 3.17: Reconstituting the sieving function of MBP in a biomimetic assay. (A) Schematic view of membrane-anchored GFP (R3-GFP). (B) Supported lipid bilayers (SLBs) mimicking myelin inner leaflet lipid composition were prepared using mol\% of following lipids: $44 \%$ cholesterol, $27 \%$ 1,2- dioleoyl-sn-glycero-3-phosphoethanolamine (PE), $2 \%$ porcine brain L- $\alpha$-phosphatidylinositol4,5-bisphosphate (PIP2), 11.5\% egg L- $\alpha$-phosphatidylcholine (PC), 12.5\% porcine brain L- $\alpha$ phosphatidylserine (PS), $3 \%$ sphingomyelin (SM), and $0.1 \%$ of 1,2-dihexadecanoyl-sn-glycero3-phosphoethanolamine (DHPE)-Texas Red. First, purified recombinant $14 \mathrm{kDa}$ MBP $(7 \mu \mathrm{M})$ was added onto the SLBs followed by the addition of GUVs composed of PS:PS in 1:2 molar ratio ( $0.1 \mathrm{~mol} \%$ of DHPE-Texas Red was used during the preparation of GUVs for visualization). MBP induced attachment of GUVs to the SLB followed by deformation and spreading. R3-GFP $(7 \mu \mathrm{M})$ was added after MBP had induced the spreading of GUVs onto the SLBs. MBP together with GUVs formed a tight barrier for the diffusion of the R3-GFP. Scale bars represent $10 \mu \mathrm{m}$. (C) Control experiments were performed as in (B) but in the absence of MBP. No GUV spreading was observed onto the SLB and R3-GFP was uniformly distributed throughout the SLB (Note that all the recombinant proteins were purified in collaboration with S. Frey at Max Planck Institute for Biophysical Chemistry, Göttingen).

that react against the lipid, sulfatide. Sulfatide was incorporated into both, SLBs (44 mol\% cholesterol, 15 mol\% PE, 2 mol\% PIP2, 11.5 mol\% PC, 12.5 mol\% PS, $3 \mathrm{~mol} \% \mathrm{SM}$ and $12 \mathrm{~mol} \%$ sulfatide) as well as GUVs (50 mol\% PC, $25 \mathrm{~mol} \% \mathrm{PS}$ and $25 \mathrm{~mol} \%$ sulfatide). First, we added O4 antibodies to the GUV solution. Antibodies clearly induced aggregation of GUVs (Figure 3.19 A). Next, we prepared SLB-antibody-GUV sandwich as described before. Importantly, neither spreading of GUVs nor extrusion of R3-GFP was observed (Figure 3.19 B). As a positive control, we used MBP, which not only induced GUV spreading, but also caused R3GFP extrusion (Figure 3.19 C). Together these experiments demonstrate that mere cross-linking of two membrane surfaces by an antibody is not sufficient to mimic the function of MBP. 

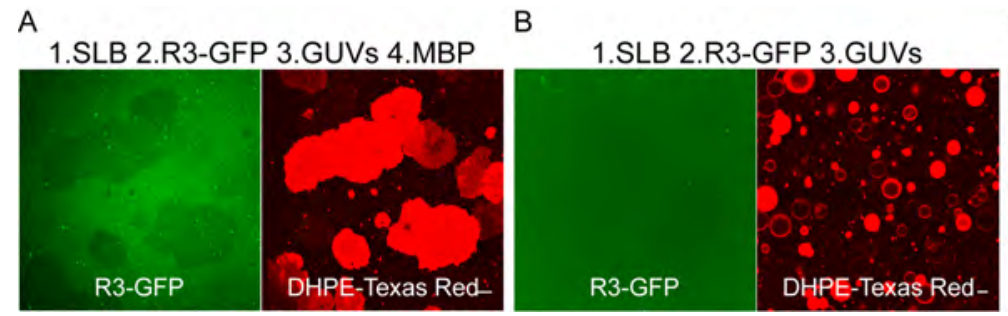

C

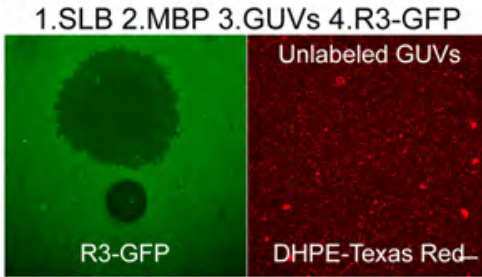

Figure 3.18: Extrusion of R3-GFP from the areas where GUVs spread onto the SLB in the presence of MBP. (A) Recombinant R3-GFP ( $7 \mu \mathrm{M})$ was added onto the SLBs, followed by the addition of purified recombinant $14 \mathrm{kDa} \mathrm{MBP}(7 \mu \mathrm{M})$ and finally GUVs were added. MBP not only induced the spreading of the GUVs onto the SLB, but also caused extrusion of R3-GFP from these areas. (B) Control experiments were performed as in (A) but in the absence of MBP. (C) As a control for quenching, SLB experiments were performed with non-labelled GUVs. Supported lipid bilayers (SLBs) mimicking inner leaflet myelin lipid composition were prepared and visualized using 0.1 mol\% of DPPE-Texas Red. Purified $14 \mathrm{kDa}$ MBP $(7 \mu \mathrm{M})$ was added to the SLB, followed by the addition of GUVs composed of PS:PC (1:2 mol\%) and R3-GFP $(7 \mu \mathrm{M})$. Scale bars represent 10 $\mu \mathrm{m}$.
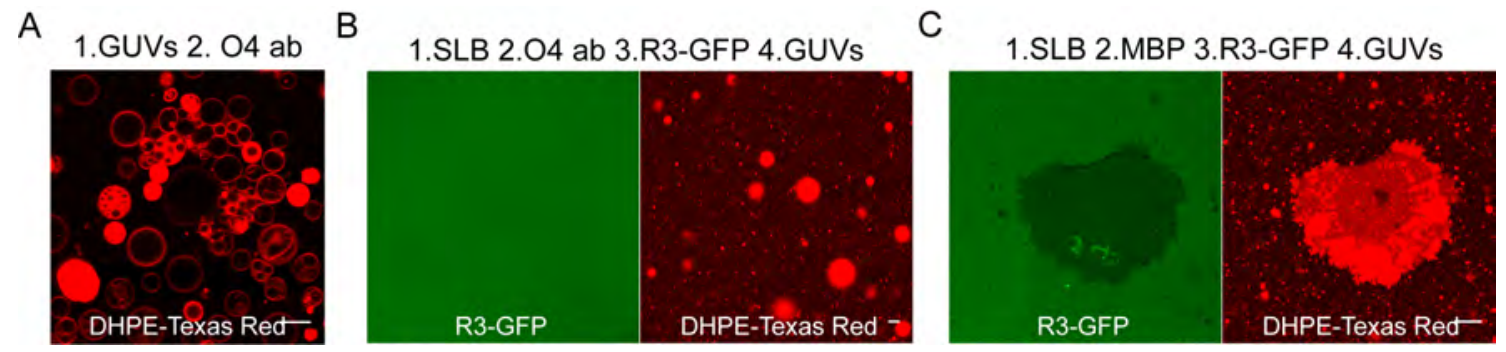

Figure 3.19: (A) O4 antibodies against the lipid sulfatide were added to GUVs composed of PC:PS:Sulfatide (2:1:1 mole \%, visualized using 0.1 mole\% DHPE-Texas Red). Addition of O4 antibodies $(10 \mu \mathrm{g} / \mathrm{mL})$ induced aggregation of GUVs. (B) Supported lipid bilayers (SLBs) using the following lipid composition were prepared: 44\% cholesterol, 15\% PE, 2\% PIP2, 11.5\% PS, $3 \%$ SM, $12 \%$ porcine brain sulfatide and $0.1 \%$ of DHPE-Texas Red. O4 antibodies $(10 \mu \mathrm{g} / \mathrm{mL})$ were added to the SLBs followed by the addition of GUVs composed of PC:PS:Sulfatide (2:1:1 mole\%, visualized using 0.1 mole\% DHPE-Texas Red). Even after repeated washing steps GUVs remained bound to the SLBs, however no extrusion of R3-GFP was observed. (C) Control experiments were performed with MBP $(7 \mu \mathrm{M})$ instead of $\mathrm{O} 4$ antibodies as in (B). MBP induced both spreading of GUVs as well as extrusion of R3-GFP. Scale bars represent $10 \mu \mathrm{m}$. 


\subsection{Sieving function of MBP requires molecular self-assembly}

\subsubsection{Displacement of bulky proteins from the myelin mem- brane sheets}

As experiments with SLBs suggested an extrusion/displacement mechanism, we next proceeded to investigate whether this also holds true in cultured oligodendrocytes. First, we looked at the distribution of non-compact myelin proteins during oligodendrocyte development. Cells were fixed between 2-7 DIV and stained for CNPase and MAG, the non-compact myelin markers (Figure 3.20 A, B). Indeed, CNPase and MAG were distributed throughout the myelin membrane sheets in 2 DIV primary oligodendrocytes (except in small regions that had strong MBP signal). However, both CNPase and MAG were restricted mostly to the processes between 4-5 DIV, time during which MBP was distinctly visible in the form of small domains within the myelin membrane sheets. As we looked further during development, between 6-7 DIV, minor veins within processes disappeared together with the formation of larger MBP domains; and bulky proteins were restricted mostly to the major veins. As a control, we looked at the distribution of MOG, the myelin membrane sheet protein, which has a small cytoplasmic domain (less than 30 amino acids). In a striking contrast to CNPase and MAG, MOG remained distributed into the membrane sheets throughout development (Figure 3.20 C, D).

Next, we investigated CNPase distribution in vivo during early myelination phase (P 14). Similar to the in vitro observations, CNPase could be clearly detected in myelin sheath in the areas that were not still compacted (Figure 3.21 A). However, as indicated in previous experiments, at P21, CNPase was mainly localized to the inner tongue areas within the non-compact myelin (Figure 3.21 B). Together, these experiments suggest an extrusion mechanism in which MBP pushes proteins with bulky cytosolic domains out of the membrane sheaths. 

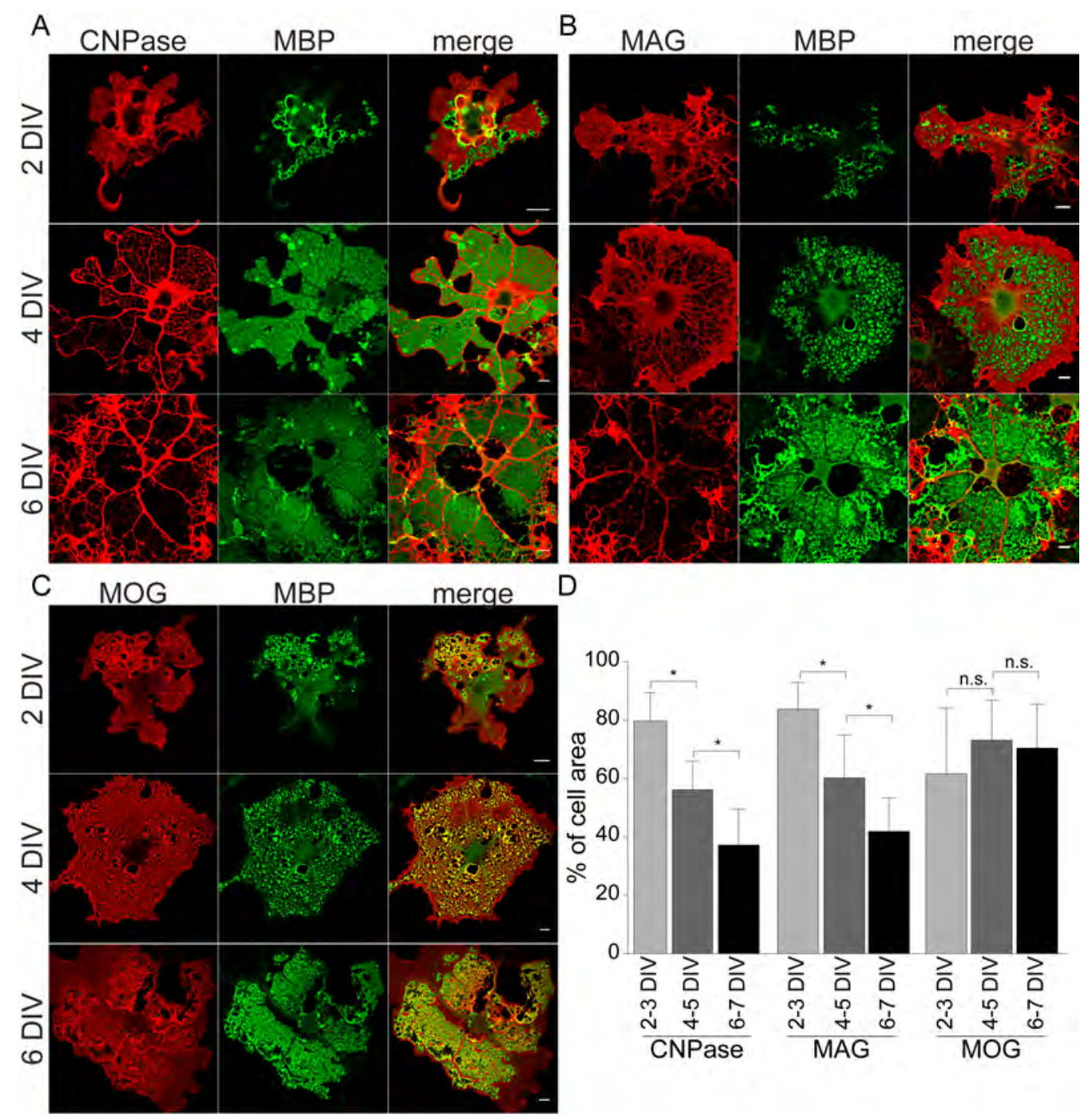

Figure 3.20: Extrusion of non-compact myelin proteins from myelin membrane sheets during oligodendrocyte development. Oligodendrocytes were immunostained for MBP (green) and (A) CNPase or (B) MAG (green) at different days in vitro (DIV), between 2 DIV and 7 DIV. In young cells (2-3 DIV), CNPase and MAG are distributed throughout the membrane sheets. Scale bars represent $10 \mu \mathrm{m}$. As the cells matured with progressive accumulation of MBP (4-7 DIV), CNPase and MAG are extruded from the sheets and localize to the processes instead. (C) MOG with a short cytoplasmic domain remains distributed into the sheets in mature oligodendrocytes. (D) Quantification of the \% of cell area occupied by the indicated protein at different DIVs. Bars show mean $\pm S D$ ( $n=40$ cells, ${ }^{*} p<0.05$, ANOVA, n.s. indicates no significance difference). 
A CNPase staining at P14 B

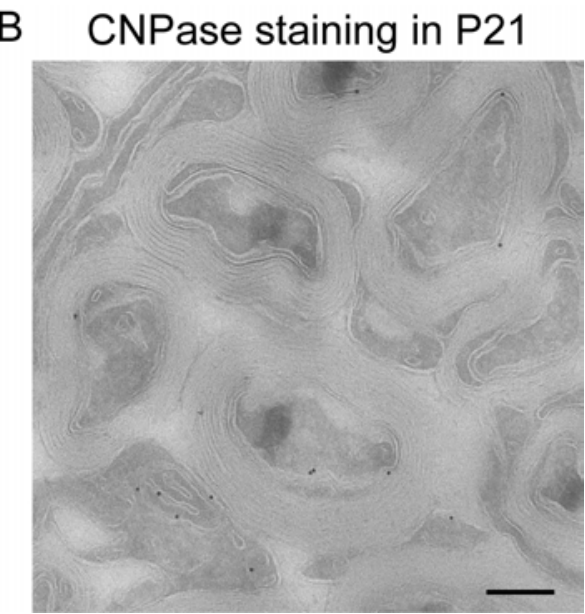

Figure 3.21: In vivo localization of CNPase in myelin during development. Immunoelectron microscopy analysis of optic nerves from wild type mice at P14 (before MBP expression) and P21 (after MBP expression). CNPase antibodies were used to investigate its localization in myelin. Scale bar in the left panel represents $500 \mathrm{~nm}$ and the scale bar in the right panel represents 200 $\mathrm{nm}$. Note that these experiments were performed in collaboration with N. Snaidero, Max Planck Institute of Experimental Medicine, Göttingen.

\subsubsection{Reconstitution of MBP barrier in a fibroblast cell line}

To further validate the extrusion of bulky proteins by MBP, we next established MBP barrier in a cell line at the interface of endoplasmic reticulum and plasma membrane (ER-PM). We chose PtK2 cells (derived from male Potorous tridactylus, also known as rat kangaroo) for the assay due to their flat morphology. It has been shown in the past that flat cell lines are suitable for visualizing ER-PM contacts/domains in mammalian cells (Várnai et al., 2007; Ercan et al., 2009). We designed a reporter construct where C-terminal domain of a non-compact myelin protein, Tmem10 was replaced with MBP (Tm10-MBP) and GFP was fused to its N-terminal side, immediately after the signal sequence (GFP-Tm10-MBP, see the schematic in Figure 3.22 A). Expression of this construct in PtK2 cells resulted in altered ER morphology. MBP coupled to Tm10 was visible in the form of distinct patch-like structures with shapes ranging from dot-like appearance to large rectangular regions (Figure 3.22 B). Localization of surface GFP molecules by immunofluorescence microscopy revealed that these patches were intracellular (Figure 
$3.22 \mathbf{C})$. Furthermore, we performed immunostainings against GFP tag in permeabilized cells. The domains were clearly stained by these antibodies (Figure 3.22 D), further confirming their intracellular localization. MBP, being a highly basic protein, has been shown to associate with the negatively charged lipids. It is this interaction of MBP with lipids which might recruit ER in close proximity to the PM, thereby restricting GFP-Tm10-MBP to the peripheral ER domains. In order to test the role of plasma membrane charge on the morphology of domains, we treated PtK2 cells expressing GFP-Tm10-MBP with $10 \mu \mathrm{M}$ of the ionophore, ionomycin. Treatment with ionomycin triggers $\mathrm{Ca}^{2+}$ influx (from extracellular medium) into the cells, which shields the surface charge (Várnai and Balla, 1998; Yeung et al., 2006; Nawaz et al., 2009). Interestingly, with the dispersal of surface charge, MBP domains retracted back into the ER (Figure 3.22 E, F). Similar results were obtained with the membrane permeant base sphingosine (Yeung et al., 2008). These experiments with surface charge alteration further confirm the role of MBP and negative charged lipids in the establishment of peripheral ER-PM contacts.

The experiments above revealed formation of ER-PM contacts in PtK2 cells upon C-terminus fusion of MBP to an integral membrane protein. The next question was whether MBP acts as a diffusion barrier for the plasma membrane proteins in these domains. To test this possibility, we first co-expressed plasma membrane targeted mem-RFP (anchored to the plasma membrane with N-terminus of neuromodulin GAP-43) together with GFP-Tm10-MBP. Indeed, mem-RFP was excluded from these ER-PM domains (Figure $3.23 \mathbf{A}$ ). As an important control, we also expressed only the Tm10 with N-terminal GFP (GFP-Tm10) into the cells together with mem-RFP (Figure 3.23 A lower panel). Both proteins were uniformly distributed throughout the plasma membrane without any visible domain formation (for quantification see Figure 3.23 B). However, does this visible ER-PM domain formation depends specifically on the transmembrane domain of Tm10? To check whether MBP forms these ER-PM contacts independent of features of the integral membrane protein, we replaced Tm10 with the transmembrane domain 4 of PLP (PLPTM4). When we expressed GFP-PLPTM4-MBP construct into PtK2 cells together with mem-RFP, we observed similar ER-PM domains from which mem- 
A

B

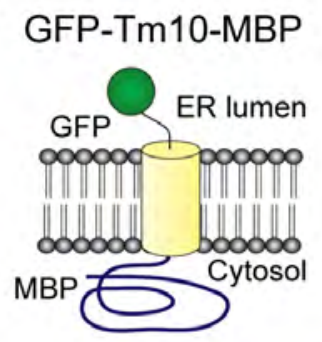

C
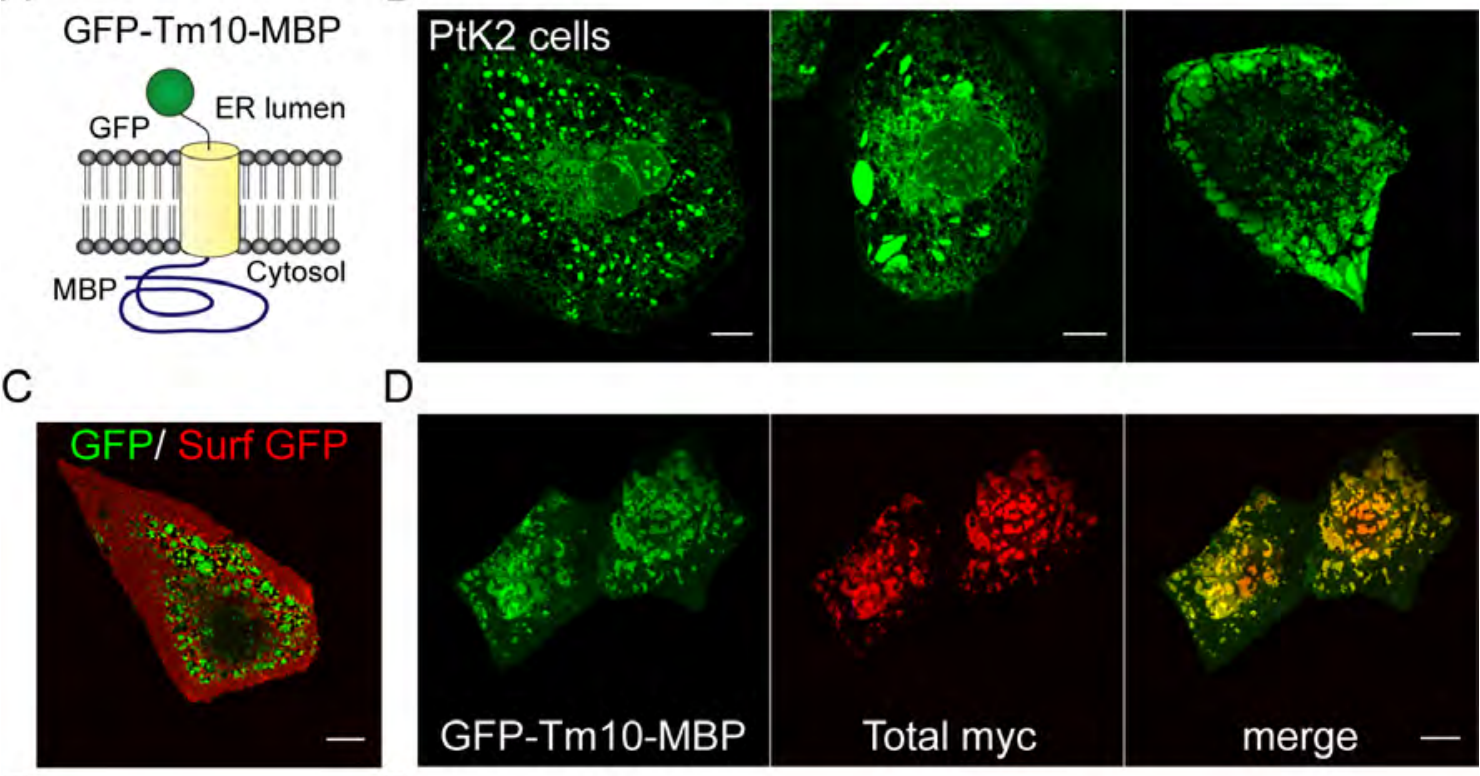

D

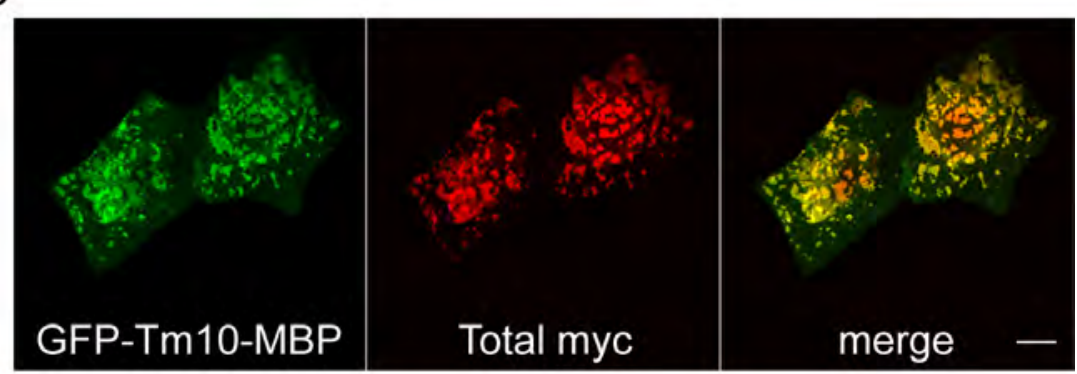

$\mathrm{E}$

$\mathrm{F}$
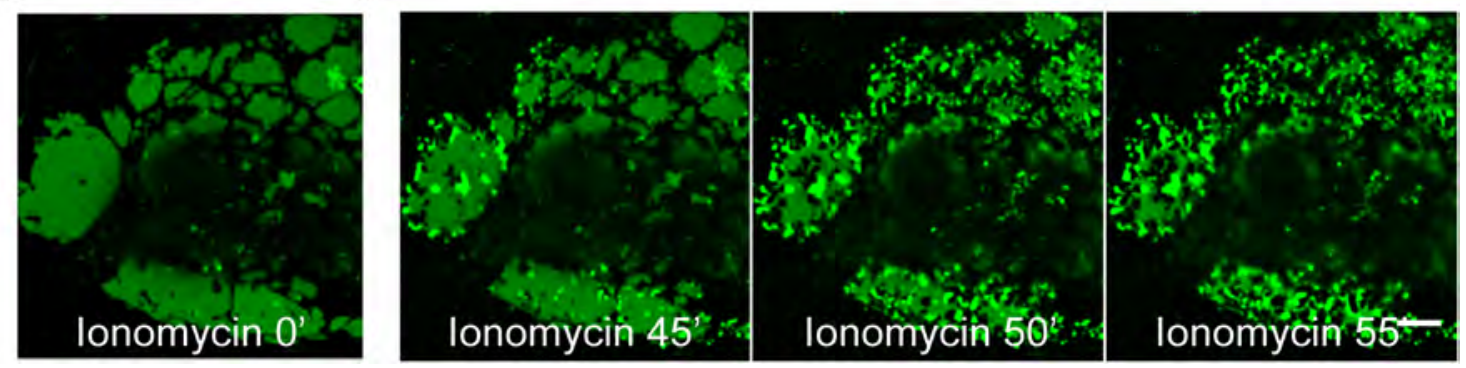

Figure 3.22: MBP establishes ER-PM domains in PtK2 cells when fused to the C-terminus of an integral membrane protein. (A) Schematic view of the reporter construct used for establishing ER-PM domains in PtK2 cells. (B) Different morphologies of MBP domains, ranging from dot-like structures emanating from the ER (left panel) to flat, rectangular sheet like structures (middle and right panel). (C) Typical image of PtK2 cells expressing GFP-Tm10-MBP (GFP in green) for 24 hrs and surface stained for the GFP molecules using antibodies (Surf GFP in red). (D) GFP can be clearly detected within MBP domains in permeabilized cells (total GFP in red channel). (E) Morphology of MBP domains before ionomycin treatment (lonomycin 0'). (F) Retraction of MBP domains into the ER with the addition of $10 \mu \mathrm{M}$ ionomycin between $45-55 \mathrm{sec}$. Scale bars in all cases represent $10 \mu \mathrm{m}$.

RFP molecules were excluded (Figure 3.23 C upper panel). Further, no domain formation or mem-RFP exclusion could be seen with only GFP-PLPTM4 (Figure $3.23 \mathbf{C}$ lower panel and $\mathbf{D}$ for quantification). Therefore, we conclude that MBP 
positive ER-PM domains form and exclude mem-RFP regardless of the features of the integral membrane proteins.
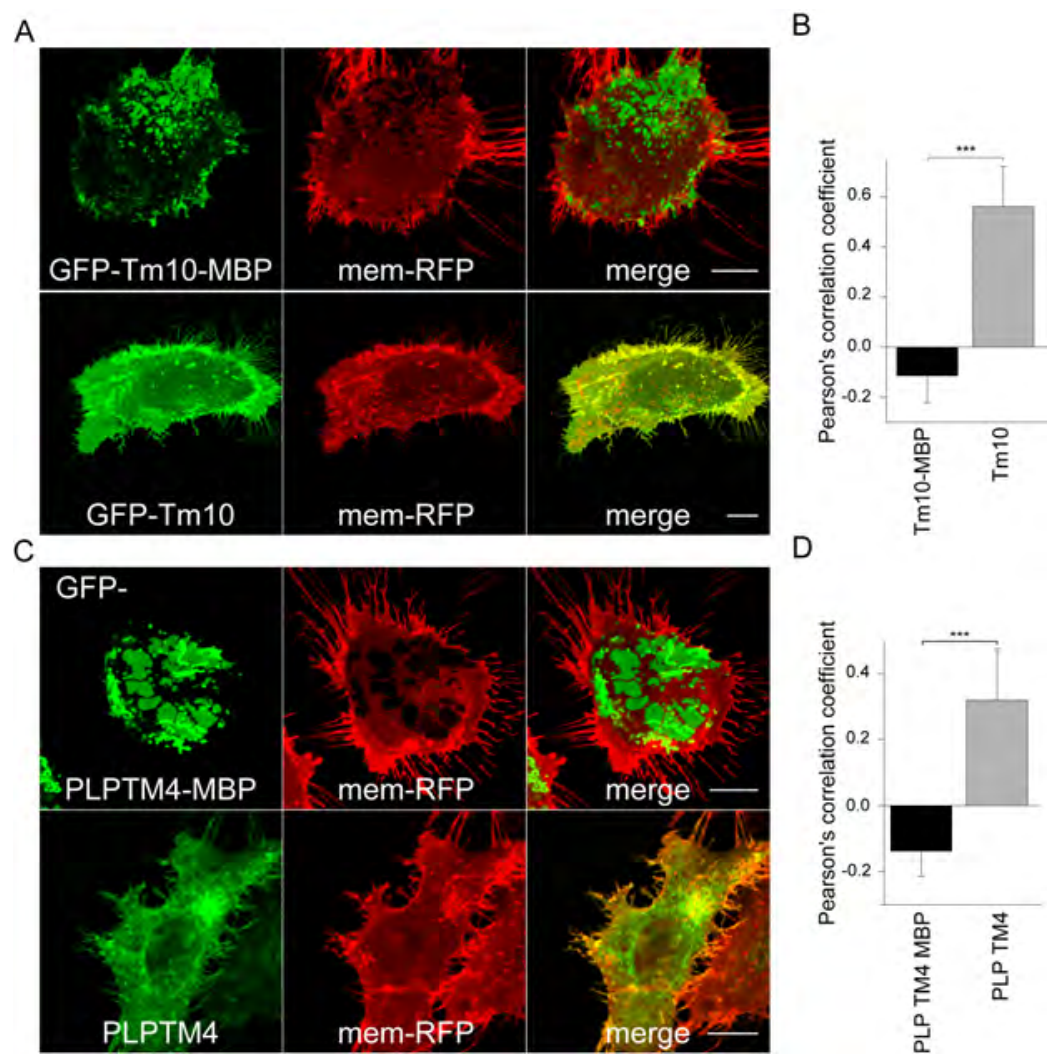

Figure 3.23: Reconstitution of MBP barrier function in a cell line. (A) PtK2 cells were cotransfected with GFP-Tm10-MBP together with mem-RFP and imaged live after 24 hrs. Expression of GFP-Tm10-MBP leads to the formation of visibly enlarged patches of ER-PM domains from which mem-RFP is excluded (upper panel). In the control sample, mem-RFP was co-transfected with GFP-Tm10. No visible domains form and mem-RFP is uniformly present throughout the plasma membrane (lower panel). Scale bars represent $10 \mu \mathrm{m}$. (B) Quantification of colocalization using Pearson's correlation coefficient. Bars show mean $\pm S D\left(n=20\right.$ cells, ${ }^{* * *} p<0.001$, $t$-test). (C) Representative images of the PtK2 cells expressing mem-RFP together with either GFP-PLPTM4-MBP (upper panel) or GFP-PLPTM4 (lower panel). Scale bars represent $10 \mu \mathrm{m}$. (D) Quantification of colocalization using Pearson's correlation coefficient. Bars show mean $\pm \mathrm{SD}$ ( $n=20$ cells, ${ }^{* * *} \mathrm{p}<0.001, t$-test). MBP forms ER-PM domains irrespective of the sequence of the transmembrane domain used. 
We next investigated whether other membrane proteins with bulky cytoplasmic domains would also be excluded from MBP positive ER-PM domains. We expressed a number of myelin proteins like PLP, CD9 and CD81, each of them containing Cterminal cytosolic GFP, together with mCherry-PLPTM4-MBP. All these proteins were excluded from MBP positive ER-PM domains (Figure 3.24). In addition, we fused GFP either to the extracellular or intracellular side of the transmembrane protein MOG. Fusion of GFP to intracellular, but not extracellular region of MOG resulted in exclusion from MBP positive domains in PtK2 cells (Figure 3.25 A, B). In primary oligodendrocytes, we observed that the cytosolic size limit for a protein to enter into the MBP positive myelin membrane sheets is less than 30 amino acids (Figure 3.9). To check whether this holds true also for MBP positive ER-PM domains in PtK2 cells, we co-expressed GFP-PLPTM4-MBP along with serial cytosolic truncations mutants of Tmem10, all expressed as N-terminal mCherry fusion proteins (Figure $3.25 \mathbf{C}$ ). As expected, only the variants with cytosolic domains of less than 30 amino acids (like Tmem10Nter, Tmem10NterC10 and Tmem10NterC20, Figure $3.25 \mathrm{D}$ ) could gain access into ER-PM domains formed by MBP. On the other hand variants of Tmem10 with 30 or 40 amino acids (Tmem10NterC30 and Tmem10NterC40 respectively) on the cytosolic domain were excluded from these MBP domains (see quantification in Figure 3.25 D).

Finally, to check the distribution of surface glycoproteins in cells forming MBP positive ER-PM domains, we stained live cells expressing mCherry-PLPTM4-MBP with fluorophore-conjugated lectins: ConA and WGA exactly as done previously in case of primary oligodendrocytes (Figure 3.13 A, C). Both these lectins were clearly excluded from the MBP positive areas as shown by the intensity profile plots (Figure 3.26 B, D). Together, these experiments convincingly demonstrate the exclusion of majority of the proteins from MBP positive ER-PM domains in PtK2 cells.

The data so far shows that MBP acts as a molecular sieve to exclude most proteins from the plasma membrane at the ER-PM contact sites in PtK2 cells. If indeed proteins are excluded from certain regions of the plasma membrane due to the barrier properties of MBP, the same proteins should have an access to these regions, 


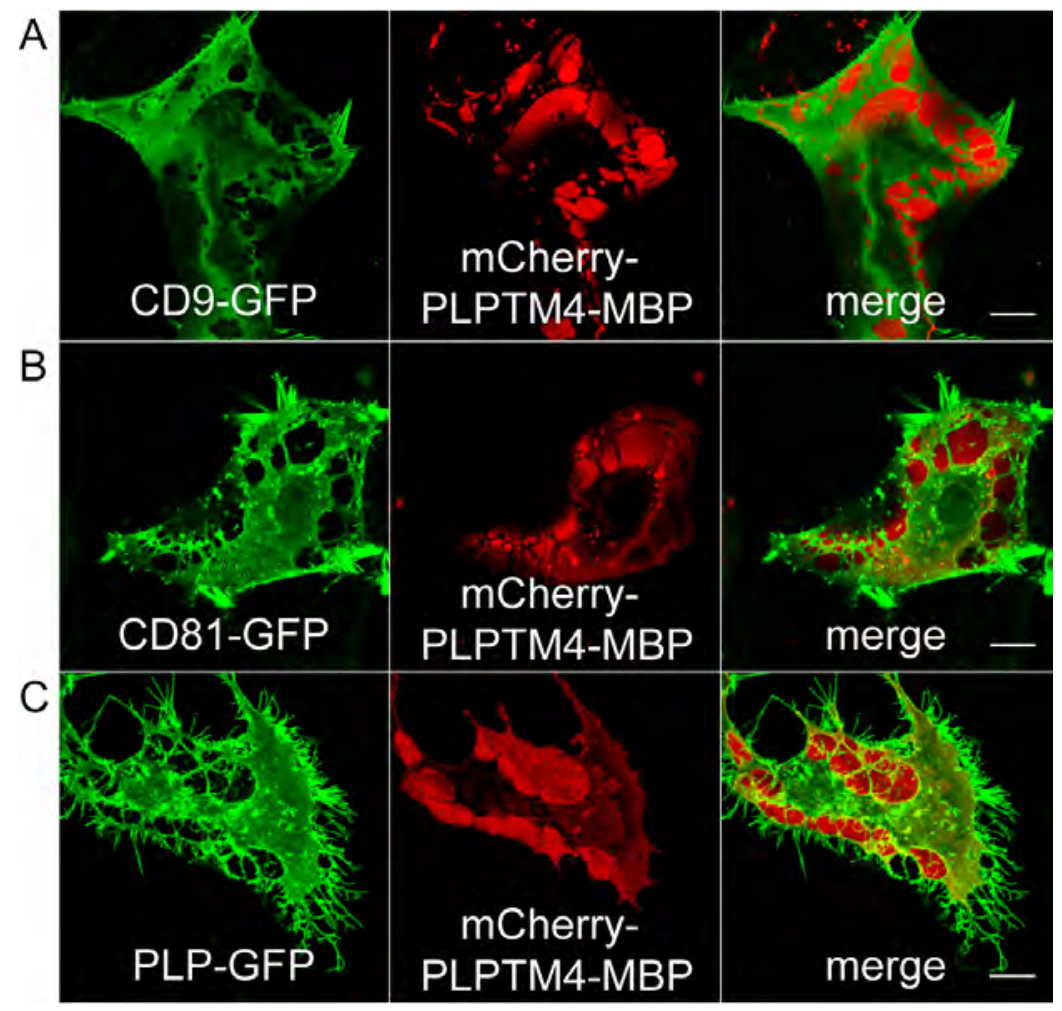

Figure 3.24: MBP positive ER-PM domains exclude proteins with bulky cytosolic domains. PtK2 cells were cotransfected with mCherry-PLPTM4-MBP and CD9-GFP/ CD81-GFP/ PLP-GFP and imaged live after $24 \mathrm{hrs}$. Scale bars represent $10 \mu \mathrm{m}$. Note that GFP/YFP in each case were fused to the cytosolic domain.

provided MBP molecules retract back from the peripheral regions of the ER. We imaged dynamics of mem-RFP in PtK2 cells co-expressing GFP-Tm10-MBP upon ionomycin (Figure 3.27 A) or sphingosine (Figure $3.27 \mathbf{B}$ ) treatment. With the retraction of MBP domains into the ER (see the white arrow heads in Figure 3.27 $\mathbf{A}$ and $\mathbf{B}$ ), mem-RFP was able to enter the previously inaccessible areas (see the white arrows Figure 3.27 A and B). This experiment clearly shows that it is the molecular sieve formed by MBP, which prevents proteins with bulky cytosolic domains from entering into ER-PM contact sites. 

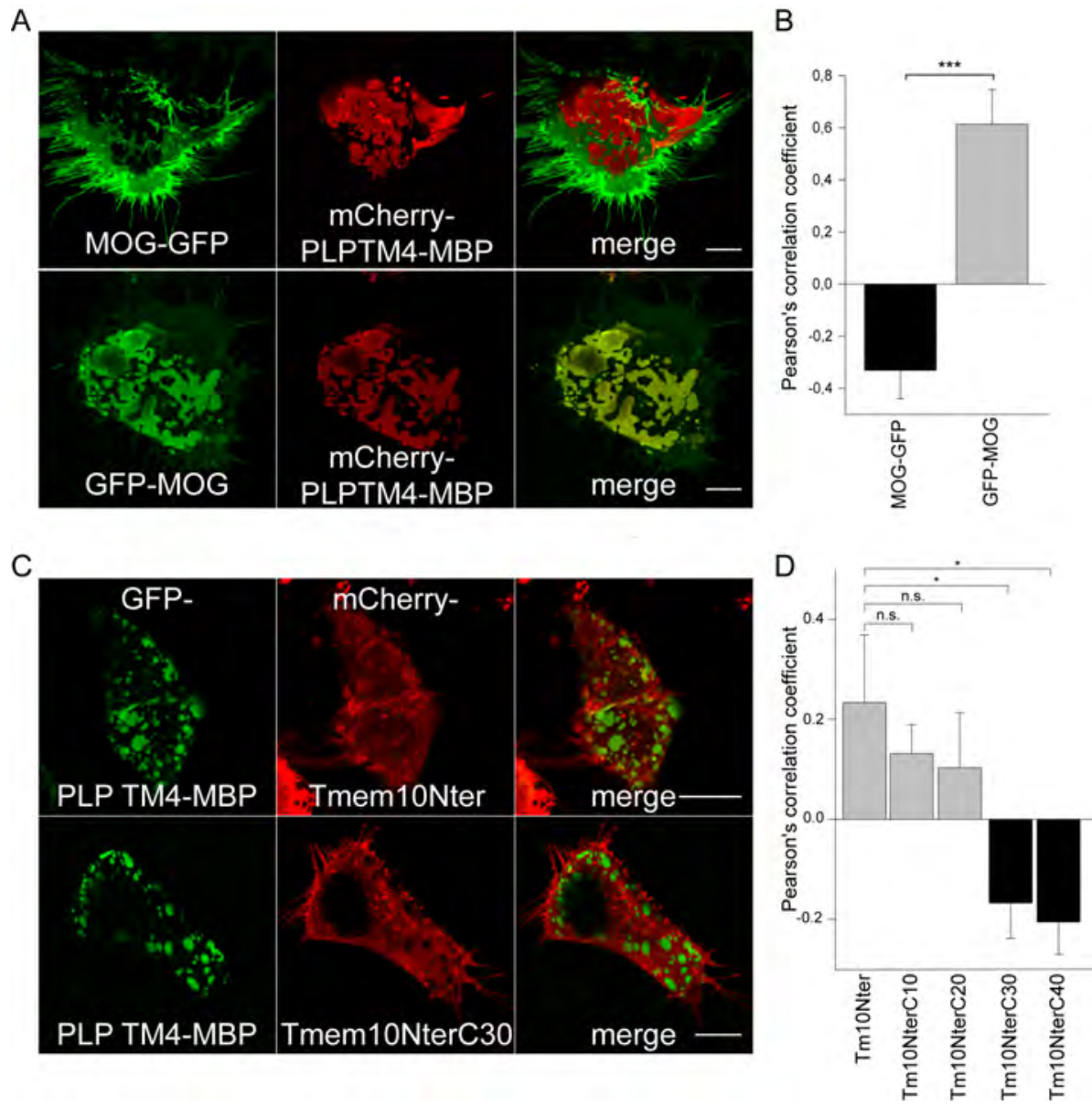

Figure 3.25: Cytosolic size limit for entering into MBP positive ER-PM domains is less than 30 aa. (A) Typical images of PtK2 cells expressing mCherry-PLPTM4-MBP together with either GFPMOG (extracellular GFP, upper panel) or MOG-GFP (intracellular GFP, lower panel). Scale bars represent $10 \mu \mathrm{m}$. (B) Quantification of colocalization using Pearson's correlation coefficient. Bars show mean $\pm S D\left(n=20\right.$ cells, ${ }^{* * *} p<0.001, t$-test). Fusion of GFP to the intracellular, but not extracellular domain leads to the exclusion of MOG from MBP positive ER-PM patches. (C) Localization of Tmem10 variants, namely Tmem10 with truncated cytosolic domain (Tmem10Nter, upper panel) and Tmem10 with 30 aa on the cytosolic side (Tmem10NterC30, lower panel) upon co-expression with GFP-PLPTM4-MBP. Scale bars represent $10 \mu \mathrm{m}$. (D) Quantification of colocalization of a series of cytoplasmic truncation mutants of Tmem10. Bars show mean \pm SD ( $n$ $=20$ cells, ${ }^{*} p<0.05$, ANOVA, n.s. indicates no significance). While Tmem10 with either truncated cytosolic domain or the cytosolic domains up to 20 aa co-exist together with MBP positive domains, 30 aa on the cytoplasmic side of Tmem10 restricts the entry into MBP positive domains. 
A

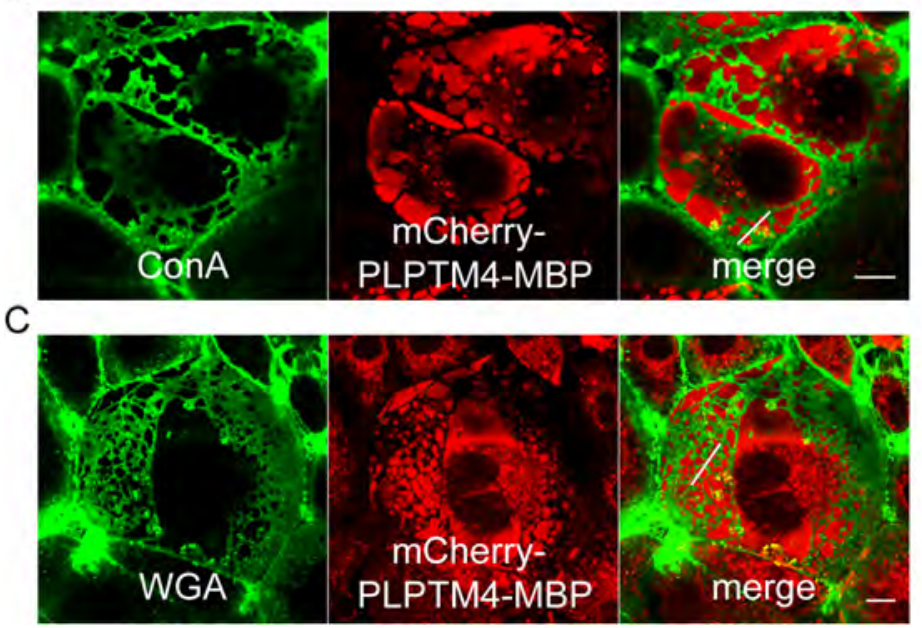

B

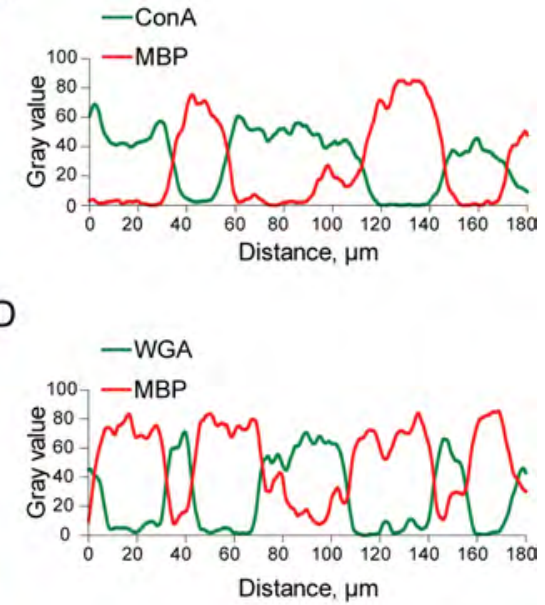

Figure 3.26: MBP positive ER-PM domains exclude glycoproteins. Typical images of lectin distribution: (A) Concanavalin A (ConA) and (C) Wheat germ agglutinin (WGA) in PtK2 cells expressing mCherry-PLPTM4-MBP. Scale bars represent $10 \mu \mathrm{m}$. Cell surface glycoproteins are excluded from MBP positive ER-PM contact sites as shown by the intensity profile plots (B, D) along the marked lines (see merged images).

\subsubsection{Self-associating assemblies of myelin basic protein}

After setting up the MBP barrier in PtK2 cells and verifying its functional properties, we next proceeded to get insights into the assembly process. The interesting question here was which properties, apart from membrane association, enable MBP to serve as an efficient size barrier. One requirement could be the formation of a tight network. Self-association between the molecular constituents of the meshwork is a plausible mechanism for achieving this. We observed that exogenously expressed MBP molecules are efficiently targeted to the mature myelin membrane sheets. This is possible only if the exogenously expressed molecules interact with the endogenous MBP molecules lining the sheets. If MBP forms two-dimensional arrays, its mobility within the network should be significantly lower in comparison to a protein that is just associated with the membrane. We employed a variety of live imaging techniques to address this question. 

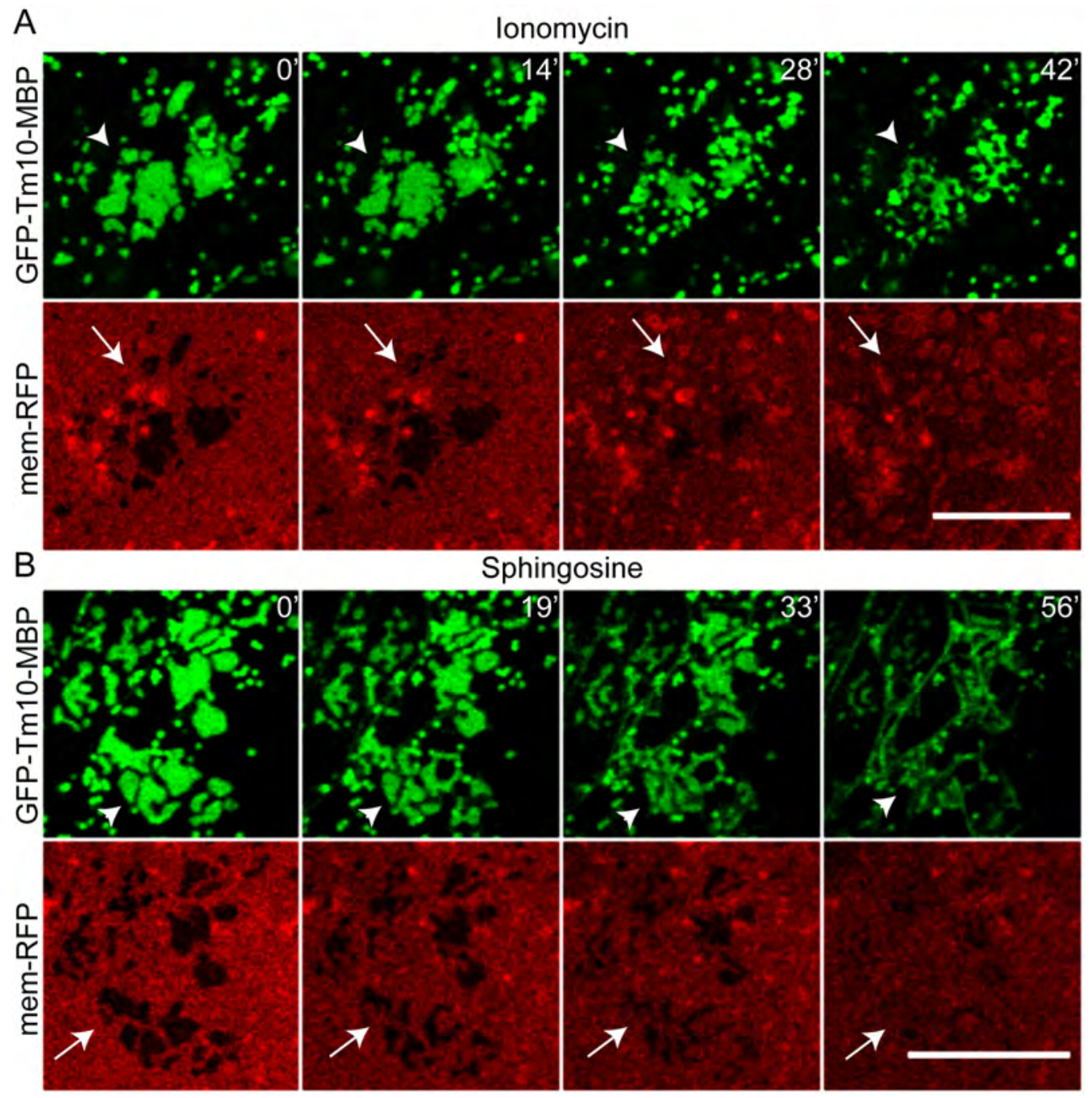

Figure 3.27: Retraction of MBP domains upon redistribution of surface charge. PtK2 cells expressing GFP-Tm10-MBP together with mem-RFP were imaged live at $37^{\circ} \mathrm{C}$. Cells were treated with either (A) $10 \mu \mathrm{M}$ ionomycin or (B) $100 \mu \mathrm{M}$ sphingosine and images were captured every 10 sec. Scale bars represent $10 \mu \mathrm{m}$. Note the uniform distribution of mem-RFP (white arrows) along the plasma membrane as MBP domains retract (white arrow heads) following surface charge redistribution.

We checked the mobility of GFP-Tm10-MBP domains in PtK2 cells and compared it with GFP-Tm10 using fluorescence recovery after photobleaching (FRAP, Figure $3.28 \mathbf{A}$ ). The Mobile fraction of GFP-Tm10 in the plasma membrane was $70 \%$, while it was only $34 \%$ for GFP-Tm10-MBP (Figure 3.28 B). The next aim was to 
measure the mobility of MBP molecules in primary oligodendrocytes. However, this is not trivial in case of primary oligodendrocytes as fusion of GFP or mCherry to MBP, either at the $\mathrm{N}$ - or $\mathrm{C}$-terminus, restricts the protein from entering into the compacted myelin membrane sheets (data not shown). To overcome this inherent difficulty, we tested the localization GFP-Tm10-MBP in primary oligodendrocytes. Interestingly, this construct efficiently localized to the membrane sheets (Figure $3.28 \mathbf{E}$ green channel). Staining for the surface GFP epitopes in non-permeabilized cells revealed that the construct was indeed efficiently targeted to the surface of myelin membrane sheets (Figure 3.28 E red channel). As an important control, we co-transfected primary oligodendrocyte cultures with Tm10-mCherry (mCherry fused to the C-terminal domain of truncated Tmem10). In a striking contrast to Tm10-MBP, Tm10-mCherry does not diffuse into MBP positive sheets (Figure 3.28 F). All together, these experiments demonstrate that GFP-Tm10-MBP maintains the barrier function of MBP. Therefore, we used this reporter construct to measure the mobility of MBP molecules. As a control, we looked at the mobility of GFPTm10, which localizes to the membrane sheets due to the truncation of cytoplasmic domain (Figure 3.28 C). Similar to PtK2 cells, mobility of Tm10-MBP was significantly lower than Tm10 (Figure 3.28 D). Low mobility of MBP molecules within the network is in agreement with results from literature (Piljić and Schultz, 2006) where self-assembling annexin A4 was shown to have significantly lower mobility in comparison to another membrane docking protein.

Next, we examined whether a protein once cross-linked into MBP domains would also exhibit lower mobility similar to MBP molecules. We chose chemically inducible molecular bridge strategy to cross-link a bulky protein like mCherry into the MBP positive domains. In this strategy, we fused the FRB fragment of mTOR protein to the N-terminal domain of Tm10-MBP after GFP (GFP-FRB-Tm10-MBP). FKBP12, other fragment of mTOR protein was fused to the N-terminus of Tm10-RFP (FKBP-Tm10-mCherry). We co-transfected PtK2 cells with these two constructs for $24 \mathrm{hrs}$ in the presence of either DMSO (control, Figure 3.29 A upper panel) or $100 \mathrm{nM}$ rapamycin (Figure 3.29 A lower panel). FRB and FKBP heterodimerize in the presence of rapamycin (Várnai et al., 2007). Live imaging experiments revealed 


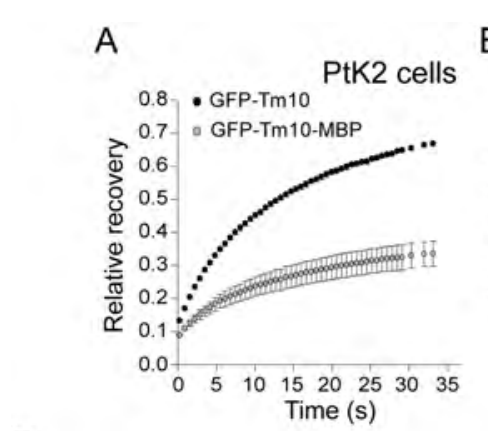

E

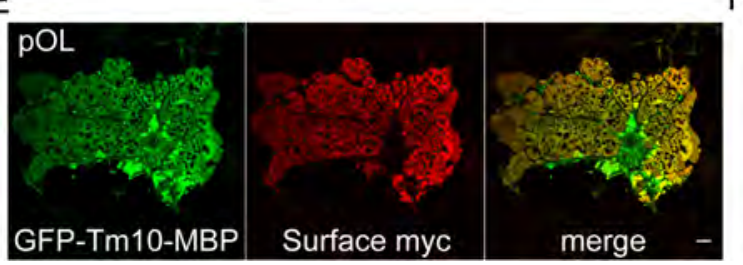

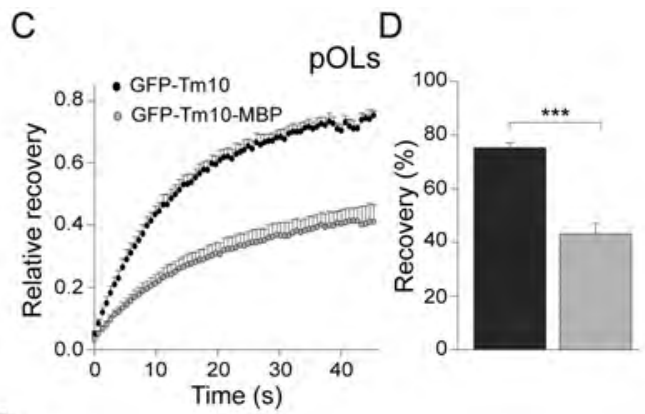

$\mathrm{F}$

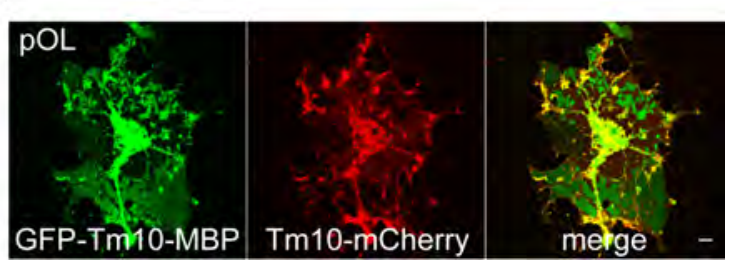

Figure 3.28: FRAP experiments to monitor the mobility of MBP domains in PtK2 cells and primary oligodendrocytes. (A) Recovery curves obtained after bleaching a squared ROI in PtK2 cells expressing either GFP-Tm10-MBP or only GFP-Tm10. (B) Average recovery after photobleaching. Bars represent mean $\pm \operatorname{SEM}\left(n=3\right.$ independent experiments, ${ }^{* * *} p<0.001, t$-test). (C) 4 DIV oligodendrocytes were transfected with GFP-Tm10-MBP or GFP-Tm10 for 16 hrs. Cells were then imaged live at $37^{\circ} \mathrm{C}$. Typical recovery curves obtained after bleaching a square $\mathrm{ROI}$ are shown as graphs. (D) Average recovery after photobleaching. Bars represent mean \pm SEM ( $n$ $=3$ independent experiments, ${ }^{* * *} \mathrm{p}<0.001, t$-test). (E) Representative images of primary oligodendrocytes expressing GFP-Tm10-MBP for $16 \mathrm{hrs}$ followed by surface staining against the GFP protein (red channel). Scale bar represents $10 \mu \mathrm{m}$. (F) Expression of GFP-Tm10-MBP preserves the barrier function of MBP as shown by exclusion of Tm10-mCherrry from the MBP positive domains within the myelin membrane sheets. Scale bar represents $10 \mu \mathrm{m}$.

that FKBP-Tm10-mCherry was excluded from MBP positive ER-PM domains in control, but not rapamycin treated cells (for quantification see Figure 3.29 B). In the next step, we compared the mobility of Tm10-mCherry outside (control recovery curve in Figure 3.29 C) vs. inside (rapamycin recovery curve in Figure 3.29 C) MBP positive ER-PM domains. Mobility of Tm10-RFP was significantly lower inside the MBP domains (For quantification see Figure 3.29 D). As a positive control, we also monitored the mobility of MBP domains (MBP recovery curve in Figure 3.29 C and Figure 3.29 D for quantification). 

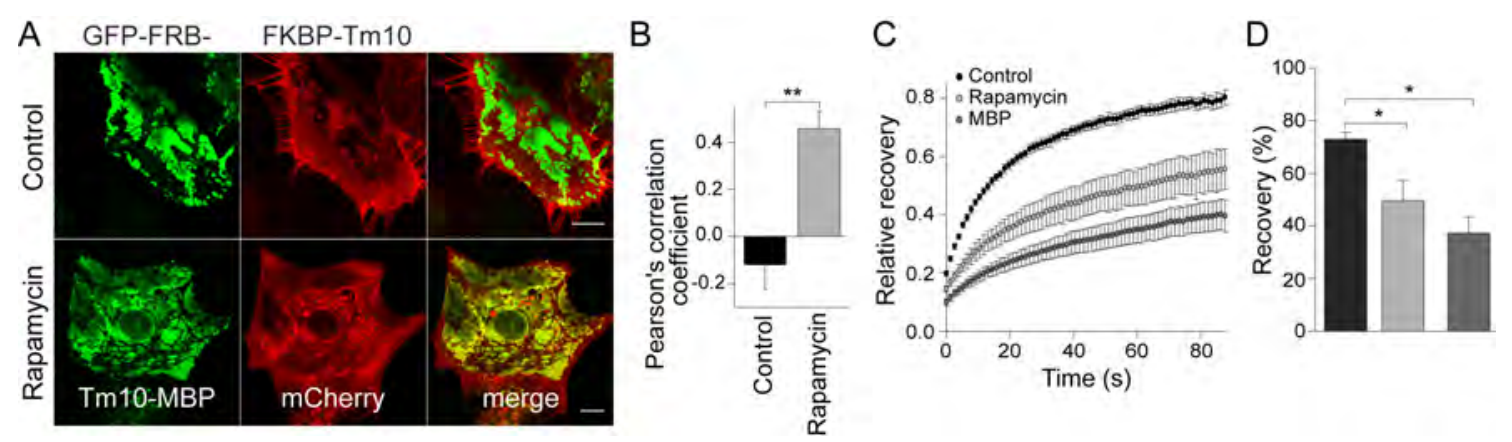

Figure 3.29: Cross-linking RFP into MBP positive domains and measuring its mobility. PtK2 cells were co-transfected with FKBP-Tm10-mCherry and GFP-FRB-Tm10-MBP for 24 hrs in the absence (Control) or presence of $100 \mathrm{nM}$ rapamycin. (A) Representative images showing distribution of two proteins in the Control (upper panel) and Rapamycin (lower panel) treated sample. Scale bars represent $10 \mu \mathrm{m}$. (B) Quantification of colocalization of mCherry within MBP positive domains in the Control vs. Rapamycin treated sample using Pearson's correlation coefficient. Bars show mean $\pm \mathrm{SD}$ ( $\mathrm{n}=20$ cells, ${ }^{* *} \mathrm{p}<0.01, t$-test). Rapamycin mediated cross-linking of FRB and FKBP enriches mCherry inside MBP domains. (C) Mobility of FKBP-Tm10-mCherry was monitored outside (Control) and inside (Rapamycin) the MBP positive domains by bleaching a squared $\mathrm{ROI}$ followed by monitoring the recovery. As a positive control, mobility of MBP domains was monitored (MBP). Typical recovery curves are presented from 3 independent experiments. (D) Average recovery curves obtained after photobleaching. Bars represent mean $\pm \operatorname{SEM}(n=3$ independent experiments, ${ }^{*} p<0.05$, ANOVA.

Apart from FRAP experiments, we also performed fluorescence decay after photoconverison (FDAP) experiments using green-to-red photoswitchable fluorescent protein Dendra2. The fluorescent protein was fused to the N-terminus of either Tm10 or Tm10-MBP. We expressed these constructs in PtK2 cells. After expression, we photoconverted a $5 \mu \mathrm{m} \times 5 \mu \mathrm{m}$ region of interest (ROI) in the transfected cells via excitation with $80 \%$ of $405 \mathrm{~nm}$ laser power, 4-5 times. Then we acquired 100 frames, every $10 \mathrm{sec}$ (Figure 3.30 A). For analysis, we quantified the average fluorescent intensities in the ROI as well as in the areas outside the ROI (for background correction) with time (Figure $3.30 \mathbf{B}$ ). We found that the rate of decay was much lower for Tm10-MBP in comparison to Tm10 only (Figure 3.30 C). We obtained similar results in primary oligodendrocytes (Figure 3.30 D, E). This inability of MBP molecules to diffuse out of an individual domains further re-affirms 
the results from the FRAP experiments.

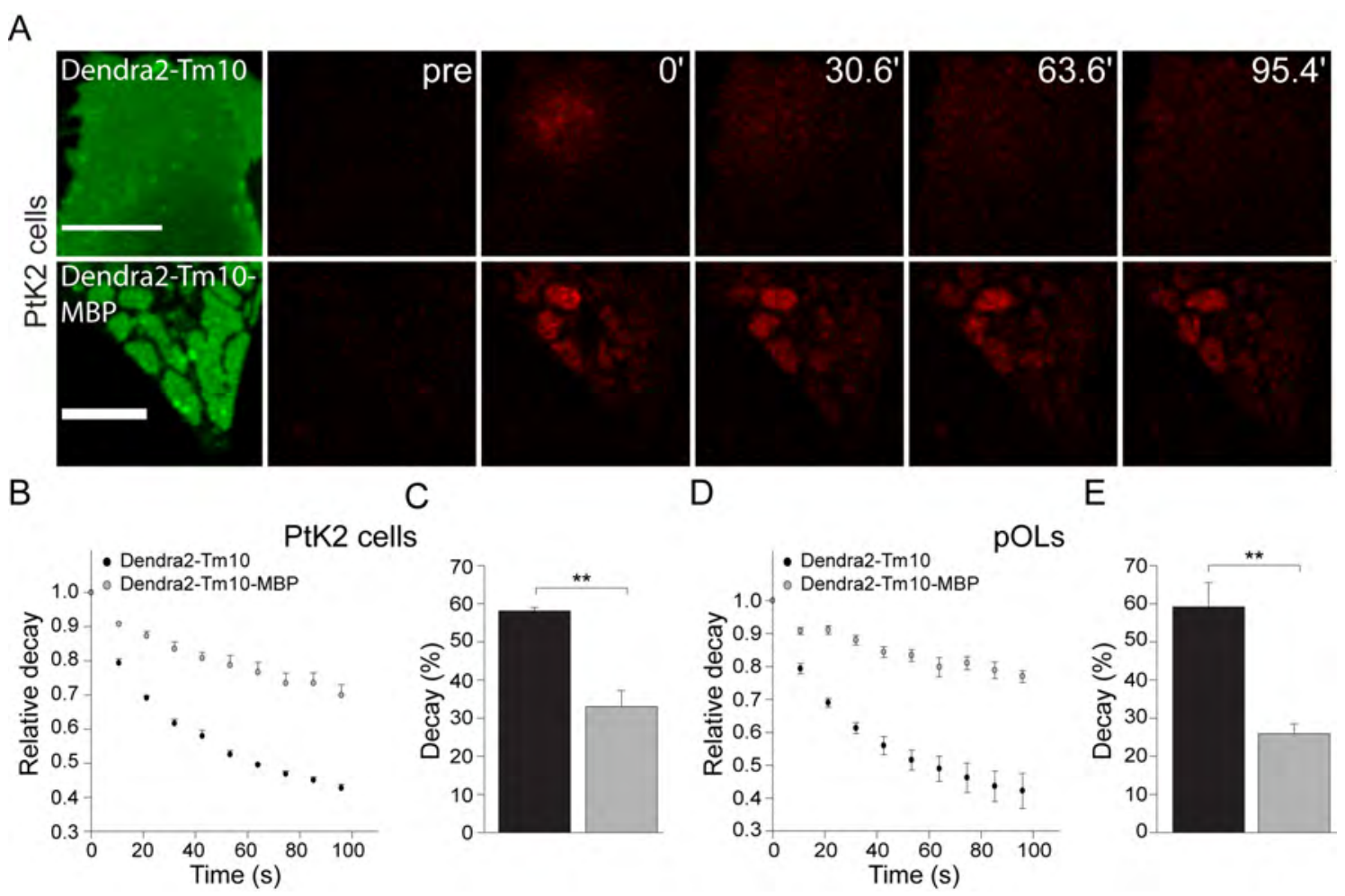

Figure 3.30: Photoconversion experiments for monitoring the mobility of MBP domains in PtK2 cells and primary oligodendrocytes. (A) PtK2 cells were transfected with either Dendra2-Tm10MBP or Dendra2-Tm10 for 24 hrs followed by live FDAP experiments. Typical images for the decay of red signal (after photoconversion) with time in cells expressing Dendra2-Tm10 (upper panel) and Dendra2-Tm10-MBP (lower panel) in the ROI. (B) Decay of signal is presented in the form of graphs from 3 independent experiments. (C) Average decay after photoconversion. Bars represent mean $\pm \operatorname{SEM}\left(n=3\right.$ independent experiments, ${ }^{* *} \mathrm{p}<0.01, t$-test). (D) Decay curves obtained from 5 DIV primary oligodendrocytes expressing either Dendra2-Tm10 or Dendra2-Tm10-MBP after photoconverting a squared ROI. (E) Average decay of signal. Bars represent mean $\pm \mathrm{SEM}$ ( $n=3$ independent experiments, ${ }^{* *} \mathrm{p}<0.01, t$-test). Signal decay is significantly less within MBP positive domains in comparison to cells expressing only the transmembrane domain.

If MBP assembles into a network, it should be possible to visualize its higher order assemblies in biochemical experiments. We performed cross-linking experiments both in vitro with minimal number of components (lipids and MBP) and in cell culture using cultures of primary oligodendrocytes. In the in vitro assay, we incubated 
MBP with GUVs (50 mole\% cholesterol, 25 mole\% PC and 25 mole\% PS) followed by the addition of various cross-linkers with variable spacer arms. Cross-linking experiments indeed revealed the formation of higher ordered assemblies of MBP molecules upon association with membranes containing negatively charged lipids. Five different cross-linkers gave comparable results (Figure $3.31 \mathrm{~A}$ ). Control experiments using the R3-GFP that also binds to the GUVs showed that MBP multimerization is specific as the R3-GFP did not cross-link into high ordered oligomers (Figure 3.31 A lower panel). Cross-linking experiments in 5 DIV primary oligodendrocytes also yielded similar results (Figure 3.31 B). All together, we conclude from these experiments that MBP molecules form higher order assemblies with low mobility on the plasma membrane.

Although the experiments above suggest for high ordered assemblies of MBP, the question was whether oligomerization is important for the sieving function. To address the possible role of oligomerization in molecular sieving, we designed a reporter construct using monomeric Plekstrin homology $(\mathrm{PH})$ domain from PLC $\delta 1$ (Klein et al., 1998). This domain has been shown to specifically bind to the negatively charged lipid PIP2 in the membrane (McLaughlin et al., 2002). We fused the PH domain to the oligomeric transmembrane domain 4 of PLP (Ng and Deber, 2010) and further cloned GFP to the N-terminal side of the construct (GFP-PLPTM4-PH). Next, we expressed GFP-PLPTM4-PH construct in PtK2 cells for 24 hrs together with mem-RFP. Surprisingly, we not only observed PH domain positive ER-PM domains (Figure 3.32 A left panel) that were similar to MBP domains (Figure 3.32 A right panel), but also the exclusion of mem-RFP from these domains.

To test the role of oligomerization in sieving function, we replaced PLPTM4 with monomeric domains. We used two sequence independent transmembrane domains that have been reported to be monomeric in nature. First domain was the mutant glycophorin domain, GypTM (Lemmon et al., 1992) and second was the mutant BNIP3 domain, BNIP TM (Sulistijo and MacKenzie, 2006). Interestingly, replacement of PLPTM4 with either of these monomeric transmembrane domains led to the uniform distribution of mem-RFP along the plasma membrane since $\mathrm{PH}$ domain 

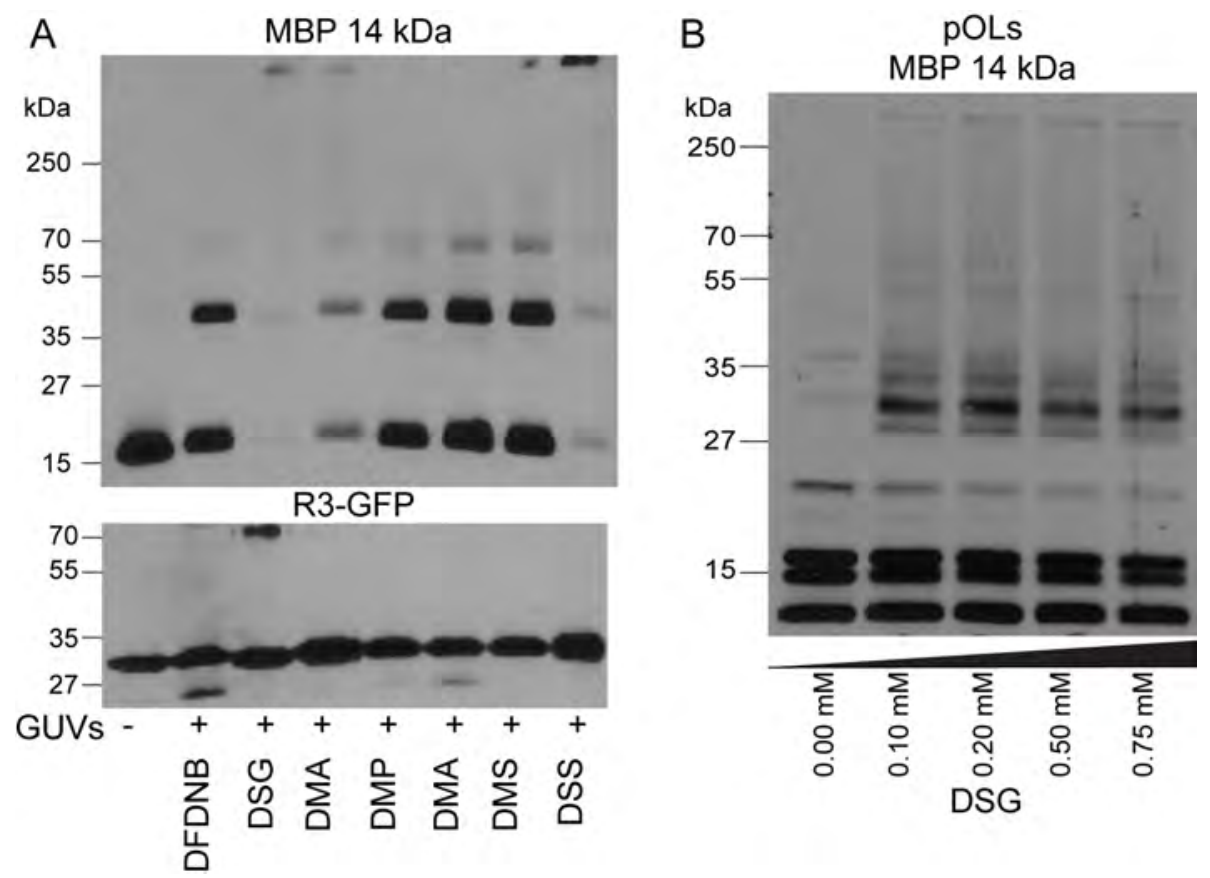

Figure 3.31: MBP forms higher order structures in vitro as well as in primary oligodendrocytes. (A) $200 \mathrm{ng}$ MBP was added to a solution of GUVs (PC:PS-2:1 mole\%) and incubated for $30 \mathrm{~min}$ at RT followed by the addition of indicated cross-linkers at a final concentration of $30 \mu \mathrm{M}$. Crosslinking experiment was performed for $30 \mathrm{~min}$. The reaction was quenched using $1 \mathrm{M}$ glycine at a final concentration of $50 \mathrm{mM}$. Control experiments were performed in a similar way using $400 \mathrm{ng}$ of R3-GFP (lower panel). (B) Chemical cross-linking reactions were performed on 5 DIV primary oligodendrocytes. Disuccinimidyl glutarate (DSG) was used in increasing concentrations as indicated and the cells were cross-linked for $20 \mathrm{~min}$ at RT followed by quenching with $1 \mathrm{M}$ Tris- $\mathrm{HCl}, \mathrm{pH} 7.5$ at a final concentration of $50 \mathrm{mM}$ and cell lysis. Equal volumes of cell lysates were subjected to Western blotting and the blots were stained with antibodies against MBP. Crosslinking converted MBP into higher molecular weight species.

failed to establish ER-PM domains (Figure 3.32 B and C left panels). As a control, we fused MBP to these monomeric transmembrane domains (Figure 3.32 B and $\mathbf{C}$ right panels). In line with the previous data where we showed higher order assemblies of MBP in oligodendrocytes, we observed ER-PM domains formation and exclusion of mem-RFP. Together, these experiments demonstrate that oligomerization, either via the transmembrane domain (for $\mathrm{PH}$ domain) or by the protein itself (in case of MBP) is sufficient for efficient molecular sieve formation. In physiological 
context, MBP is a soluble protein that associates with the membrane. Therefore, oligomerization should therefore play a critical role in orchestrating the barrier functions of MBP.
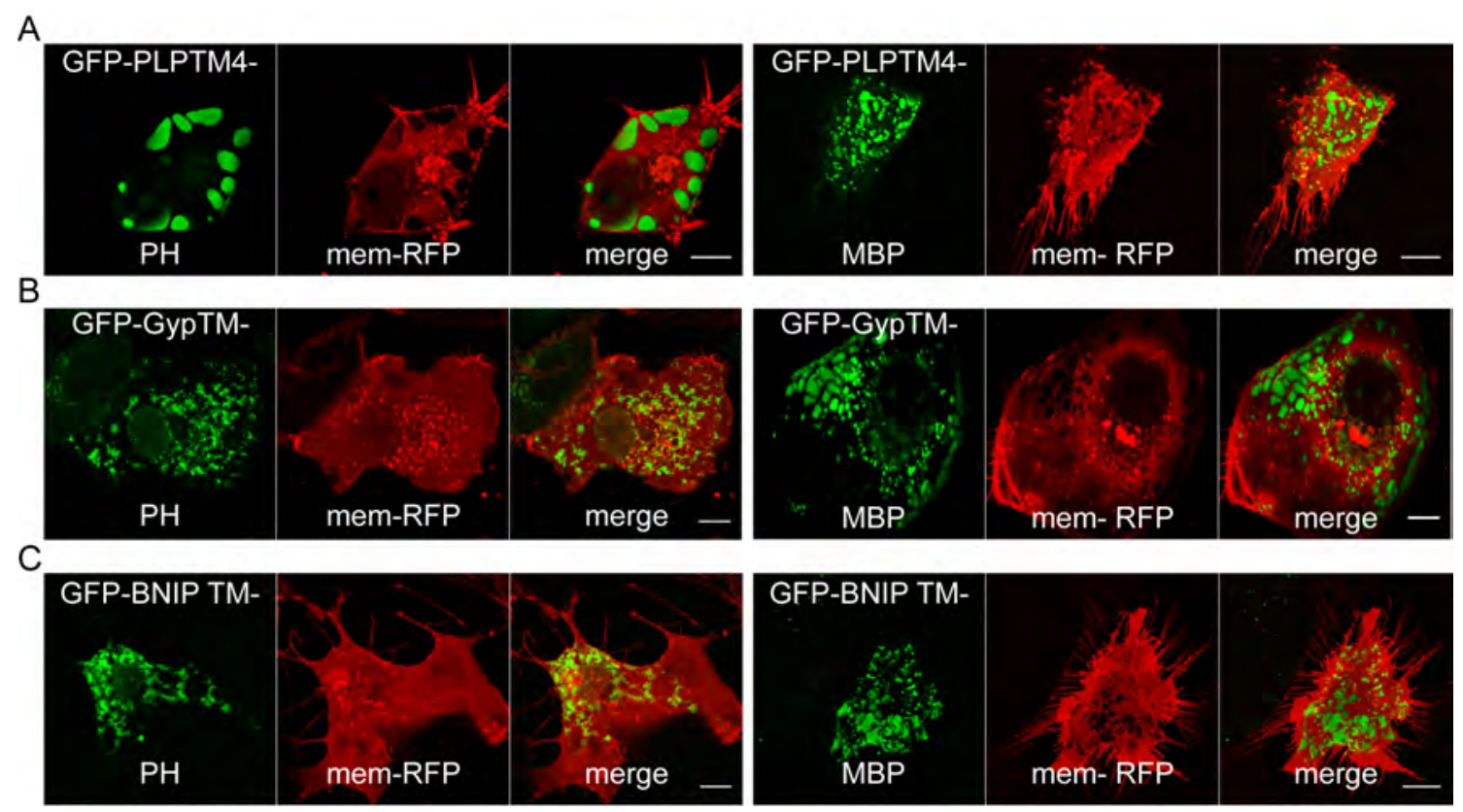

Figure 3.32: Oligomerization requirements for the efficient size barrier formation. Representative images of PtK2 cells co-expressing mem-RFP together with either PH domain (left panels) or MBP (right panels) fused to (A) oligomeric transmembrane domain 4 of PLP (PLPTM4), (B) monomeric glycophorin domain (GypTM) and (C) monomeric BNIP3 transmembrane domain (BNIP TM). Scale bars represent $10 \mu \mathrm{m}$. For the PH domain, fusion to oligomeric and not monomeric transmembrane domains leads to the formation of ER-PM domains and the exclusion of mem-RFP.

\subsubsection{Molecular nature of self-association}

Since self-assembly seems to be an important criteria for the sieving functions of MBP, we next aimed to understand the nature of self-association. Could we define specific residues within the sequence of MBP that are responsible for self-association? A very important example of physiological size-based diffusion barrier includes nuclear pore complexes, which allow only a restricted set of molecules to enter passively 
into the nucleus. Natively unfolded nuclear pore complexes form this permeability barrier by assembling into sieve like meshwork via hydrophobic interactions between the repetitive clusters of FG-repeats (Frey et al., 2006; Frey and Görlich, 2007). We tested the interesting possibility of self-association between MBP molecules through hydrophobic phenylalanine residues. We generated a mutant of MBP $14 \mathrm{kDa}$ isoform where all the phenylalanine residues (six in this case) were replaced by serines. The mutant is referred to as MBP $\mathrm{F} \rightarrow \mathrm{S}$. Next, we fused MBP $\mathrm{F} \rightarrow \mathrm{S}$ with an HA tag and expressed it exogenously into the MBP positive myelin membrane sheets. Interestingly, the mutant was restricted from entering into the sheets, unlike the wild type MBP (also fused with an HA tag). This experiment indicates that $\mathrm{F} \rightarrow \mathrm{S}$ mutant is incapable of interacting with the network.

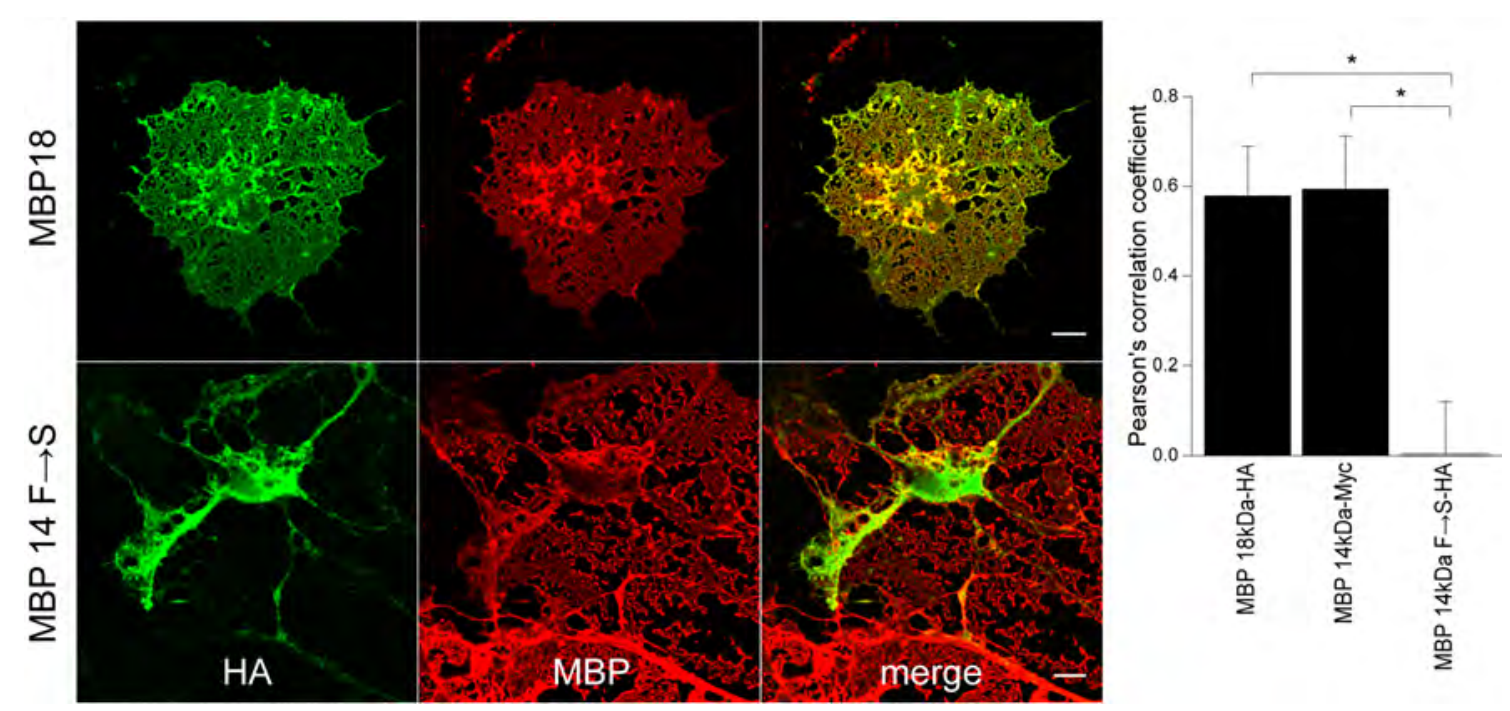

Figure 3.33: $M B P F \rightarrow S$ is restricted from entering into the myelin membrane sheets. (A) Typical images of 5 DIV primary oligodendrocytes expressing $18 \mathrm{kDa}$ isoform of wild type MBP (MBP 18) or $14 \mathrm{kDa}$ isoform of $\mathrm{MBP} F \rightarrow S$ mutant $(\mathrm{MBP} 14 \mathrm{~F} \rightarrow \mathrm{S}$ ), both tagged at the $C$-terminus with an HA tag. After 16 hrs of expression, cells were stained for MBP. (B) Quantification of colocalization of the expressed constructs with the total MBP signal using Pearson's correlation coefficient. Bars represent mean $\pm S D\left(n=20\right.$ cells, ${ }^{*} p<0.05$, ANOVA).

However, another possibility could be that the $\mathrm{F} \rightarrow \mathrm{S}$ mutant has lost the ability to bind to the membranes. We used a number of independent approached to test this possibility. 


\subsubsection{MBP $\mathrm{F} \rightarrow \mathrm{S}$ mutant retains membrane binding capabilities}

First, we expressed MBP $\mathrm{F} \rightarrow \mathrm{S}$ mutant in oligodendroglial precursor cell line, Olineu cells. $\mathrm{F} \rightarrow \mathrm{S}$ mutant clearly highlighted the plasma membrane of the cells similar to the wild type MBP (also see the intensity profile plots, Figure 3.34 A).

A

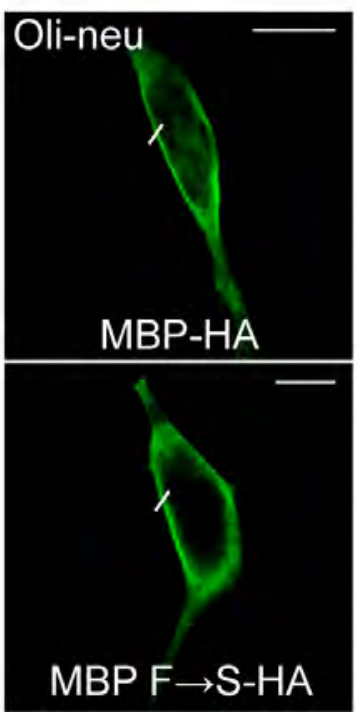

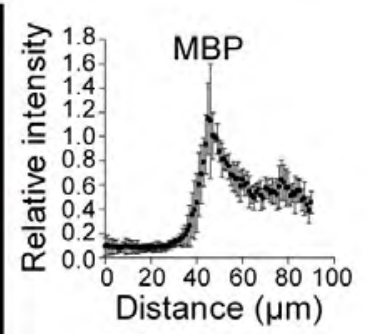

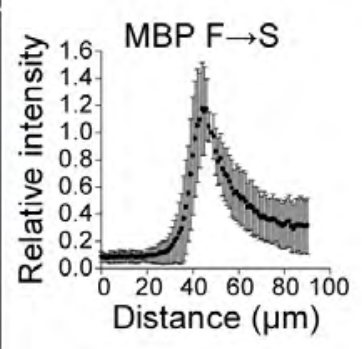

B

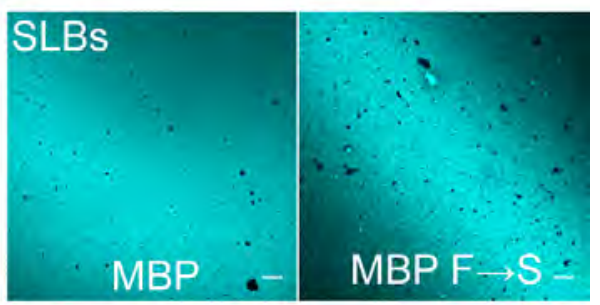

C

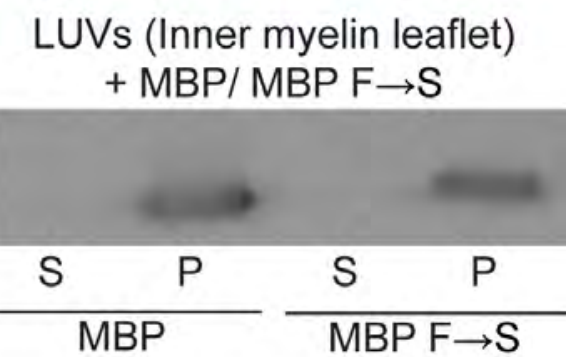

Figure 3.34: $M B P F \rightarrow S$ retains membrane binding capabilities similar to wild type MBP. (A) Representative images of Oli-neu cells transfected with either wild type (MBP-HA) or the $\mathrm{F} \rightarrow \mathrm{S}$ mutant (MBP $\mathrm{F} \rightarrow \mathrm{S}-\mathrm{HA}$ ) for $24 \mathrm{hrs}$ followed by immunostaining against the C-terminal HA tag. Scale bars represent $10 \mu \mathrm{m}$. Plasma membrane association is similar for both the wild type and the $F \rightarrow S$ mutant as shown by the relative intensity profile plots along the marked lines $(n=20$ cells). (B) Typical images of SLBs (inner myelin leaflet lipid composition) incubated with $7 \mu \mathrm{M}$ of either purified wild type (MBP) or mutant (MBP $F \rightarrow S$ ) for 10 min followed by washing and immunostaining for MBP. Scale bars represent $10 \mu \mathrm{m}$. (C) LUVs (100 nm in size and inner myelin leaflet lipid composition), incubated with $3.5 \mu \mathrm{M}$ of WT (MBP) or mutant MBP (MBP F $\rightarrow$ S) for 1 $\mathrm{hr}$, were centrifuged at 100,000 $\mathrm{g}$ for $30 \mathrm{~min}$. Pellet $(\mathrm{P})$ was resuspended in the same volume as the supernatant $(S)$ and fractions were then subjected to Western blotting. Both wild type as well as $\mathrm{F} \rightarrow \mathrm{S}$ mutant were recovered in the pellet fraction.

Second we compared the binding of purified MBP and MBP F $\rightarrow \mathrm{S}$ to the supported lipid bilayers (inner myelin membrane lipid composition). The proteins were added onto the SLBs and after extensive washing, SLBs were stained with MBP antibodies. For both, WT MBP as well as $\mathrm{F} \rightarrow \mathrm{S}$ mutant, SLBs were positively stained (Figure 
3.34 B). Third, we performed in vitro lipid vesicle co-sedimentation assay with the wild type and mutant MBP. In these experiments, $100 \mathrm{~nm}$ sized large unilamellar vesicles (LUVs) were prepared mimicking inner myelin leaflet lipid composition. LUVs were incubated with either wild type or mutant MBP for $1 \mathrm{hr}$ followed by ultracentrifugation to pellet down LUVs. MBP amounts in the pellet and supernatant were estimated by Western blotting (Figure $3.34 \mathbf{C}$ ). Again, both wild type and mutant MBP were mostly found in the pellet fraction further confirming that membrane binding remains comparable between wild type and mutant.

\subsubsection{Self-interaction between MBP molecules is promoted by hy- drophobicity per se at the site of phenylalanine residues}

Since $\mathrm{F} \rightarrow \mathrm{S}$ mutations did not affect the membrane binding properties of MBP, we could continue to explore whether it forms the molecular sieve and thereby exclude bulky proteins like wild type protein.

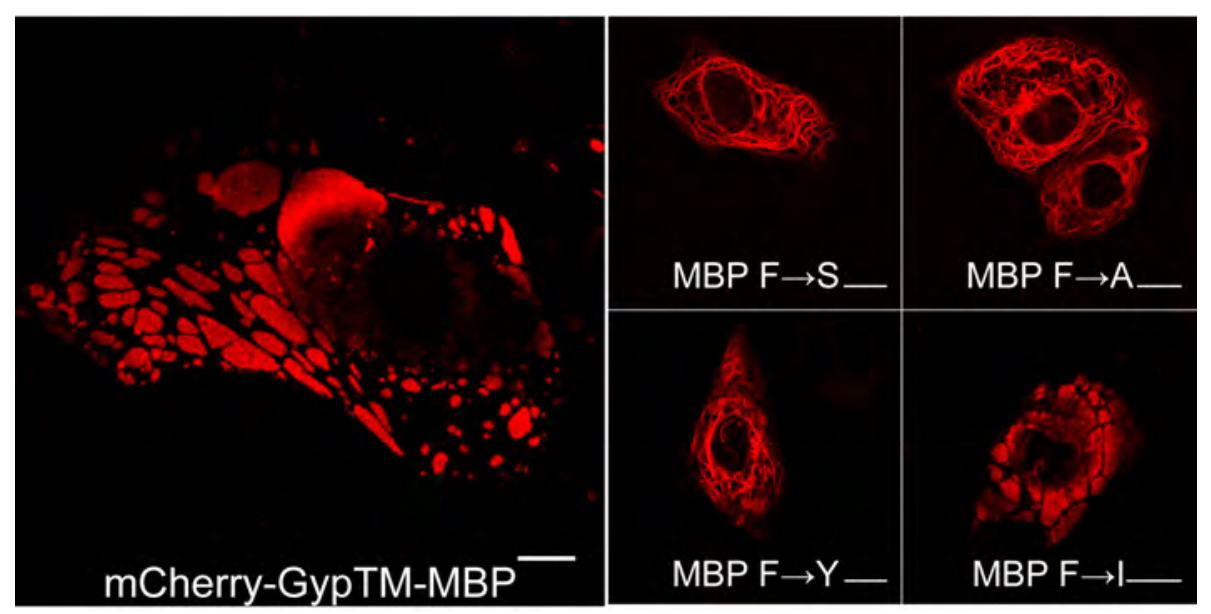

Figure 3.35: ER-PM domain formation by various MBP mutants in PtK2 cells. Typical images of the PtK2 cells transfected with either mCherry-GypTM- MBP (left most panel) or with various MBP mutants, namely $\mathrm{F} \rightarrow \mathrm{S}, \mathrm{F} \rightarrow \mathrm{A}, \mathrm{F} \rightarrow \mathrm{Y}$ and $\mathrm{F} \rightarrow \mathrm{I}$. Scale bars represent $10 \mu \mathrm{m}$. While MBP $\mathrm{F} \rightarrow \mathrm{S}$ and $\mathrm{F} \rightarrow \mathrm{A}$ fail to form the domains completely, $\mathrm{F} \rightarrow \mathrm{Y}$ shows an intermediate phenotype with reduced tendency to form domains. In a striking contrast $F \rightarrow I$ mutant forms domains similar to wild type MBP. 
We turned back to the PtK2 cell assay to compare $\mathrm{F} \rightarrow \mathrm{S}$ mutant with wild type MBP. We fused MBP $\mathrm{F} \rightarrow \mathrm{S}$ mutant to the C-terminal domain of mCherry-GypTM (Note that both mCherry as well the transmembrane domain are monomeric in nature). Next, we expressed the protein in PtK2 cells for 24 hrs followed by live imaging. As a control, we used mCherry-GypTM-MBP. Unlike wild type MBP, MBP F $\rightarrow$ S mutant did not form any ER-PM domains (Figure 3.35). One possibility of how phenylalanine residues could mediate close association between MBP molecules is via the side chains. Another way could be that the overall hydrophobicity per se at these positions promotes self-interactions. In order to distinguish between these two possibilities, we designed other mutants where hydrophobic phenylalanines were replaced with either neutral, non polar residues like glycine $(\mathrm{F} \rightarrow \mathrm{G})$ and alanine $(\mathrm{F} \rightarrow \mathrm{A})$ or hydrophobic residues like isoleucines $(\mathrm{F} \rightarrow \mathrm{I})$. Furthermore, we also designed $\mathrm{F} \rightarrow \mathrm{Y}$ mutant which has a hydrophobic aromatic ring together with polar -OH group. Indeed $\mathrm{F} \rightarrow \mathrm{G}$ as well as $\mathrm{F} \rightarrow \mathrm{A}$ mutants were incapable of ER-PM domain formation. On the other hand, $\mathrm{F} \rightarrow \mathrm{I}$ mutant efficiently formed ER-PM domains. Finally, $\mathrm{F} \rightarrow \mathrm{Y}$ mutant formed domains with drastically less efficiency than the wild type protein (Figure 3.35). Therefore, hydrophobicity per se at these positions is important for self-association.

\subsubsection{FRET measurements reveal less proximity between $\mathrm{MBP} F \rightarrow \mathrm{S}$ molecules}

As an independent approach, we performed FRET experiments in PtK2 cells to compare the proximity between $\mathrm{MBP} \mathrm{F} \rightarrow \mathrm{S}$ mutant molecules. We fused $\mathrm{F} \rightarrow \mathrm{S}$ mutant to the C-terminus of the FRET pair: GFP-Tm10 (donor) and mCherry-GypTM (acceptor). Next, we expressed GFP-Tm10-MBP F $\rightarrow \mathrm{S}$ and mCherry-GypTM-MBP $\mathrm{F} \rightarrow \mathrm{S}$ in PtK2 cells for $24 \mathrm{hrs}$ followed by fixing. We measured FRET between the acceptor and donor pair using acceptor photobleaching (Figure 3.36 A). As a negative control, we used PtK2 cells co-expressing GFP-Tm10 and mCherry-GypTM. In the positive control we replaced $\mathrm{F} \rightarrow \mathrm{S}$ with wild type MBP. FRET efficiency between the $\mathrm{F} \rightarrow \mathrm{S}$ molecules was less than two fold in comparison to either the wild type MBP (for quantification see Figure $3.36 \mathbf{B}$ ). 


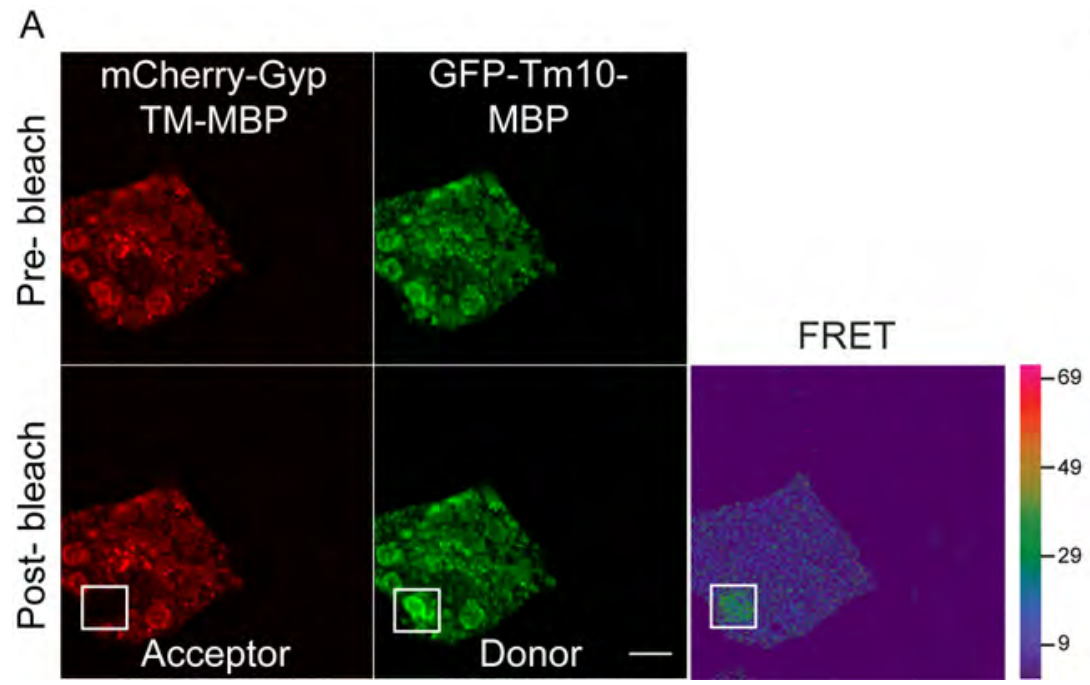

B

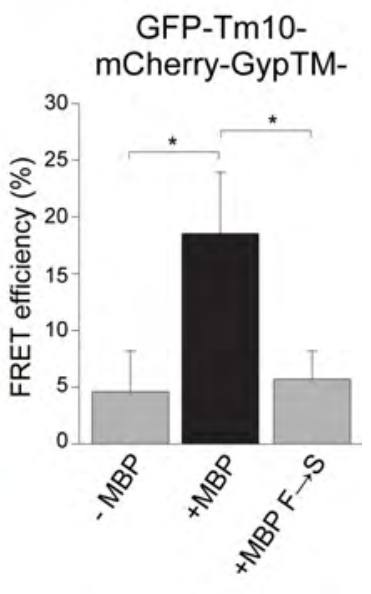

Figure 3.36: Self-interactions between wild type MBP molecules vs $\mathrm{F} \rightarrow \mathrm{S}$ mutant molecules. FRET measurements between wild type and MBP $F \rightarrow S$ mutant in PtK2 cells. PtK2 cells were cotransfected with GFP-Tm10-MBP/MBP $F \rightarrow S$ and mCherry-GypTM-MBP/MBP $F \rightarrow S$ in 1:1 ratio for $24 \mathrm{hrs}$ followed by fixing. As control, cells were co-transfected with only the two transmembrane domains: GFP-Tm10 and mCherry-GypTM. FRET efficiency was measured by performing acceptor photobleaching. (A) Typical pre-bleach (top row) and post-bleach (bottom row) images for PtK2 cells transfected with GFP-Tm10-MBP (donor) and mCherry-GypTM-MBP (acceptor). FRET signal is depicted in the pseudocolor scale (bottom row, extreme right panel). Scale bar represents $10 \mu \mathrm{m}$. (B) Quantification of FRET efficiency for the indicated pairs. Bars represent mean $\pm S D\left(n=20\right.$ cells, ${ }^{*} p<0.05$, ANOVA).

\subsubsection{Measurement of adhesion forces between wild type and $\mathrm{F} \rightarrow \mathrm{S}$ molecules}

In addition to FRET experiments, we also performed AFM measurements to monitor MBP-MBP interactions. We adsorbed MBP to the negatively charged mica surface and silicon nitride AFM tip. MBP molecules are known to bind to the mica surface (Mueller et al., 1999). In order to investigate the strength of interaction forces, we carefully moved MBP-coated cantilever tip towards MBP molecules present on the surface of mica. Next, we collected a series of approach and retraction curves (see the schematic in Figure 3.37 A). Representative curves for the approach and retraction cycles are presented in Figure 3.37 B. Retardation of peaks during the approach cycle indicated that there is little interaction during the approach cycle. However, 
as tip was retracted from MBP coated mica surface, strong adhesive forces were encountered. Control experiments, performed only in the buffer solution, revealed negligible adhesion between AFM tip and mica surface (Figure 3.37 C). Peak of the retraction curve gave the value of force required to separate the MBP-coated AFM tip from the MBP molecules present on mica surface. Multiple force curves were recorded at a given position and were highly reproducible. Force spectra were strikingly different for the mutant in comparison to the wild type MBP. Peak force measurements revealed 5-fold stronger adhesion of wild type MBP molecules (500 $\mathrm{nN}$ ) in comparison to the mutant (100 nN) (Figure $3.37 \mathbf{C})$.

A

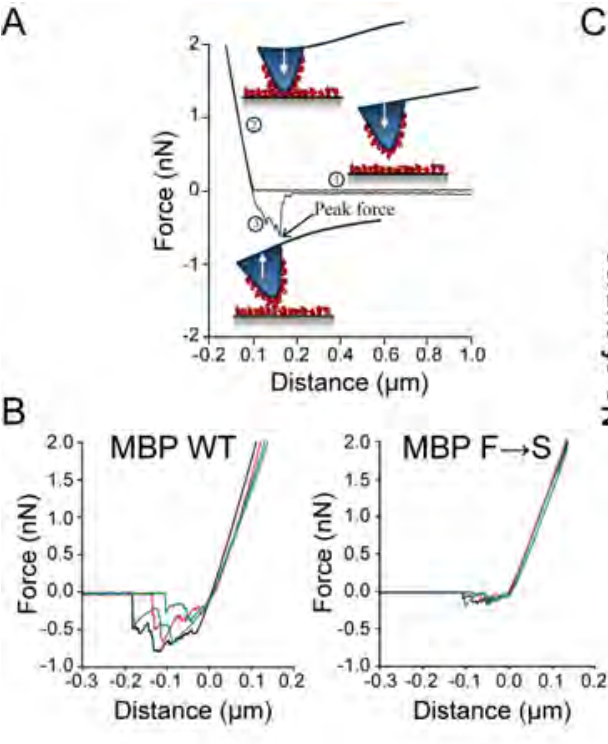

B
C

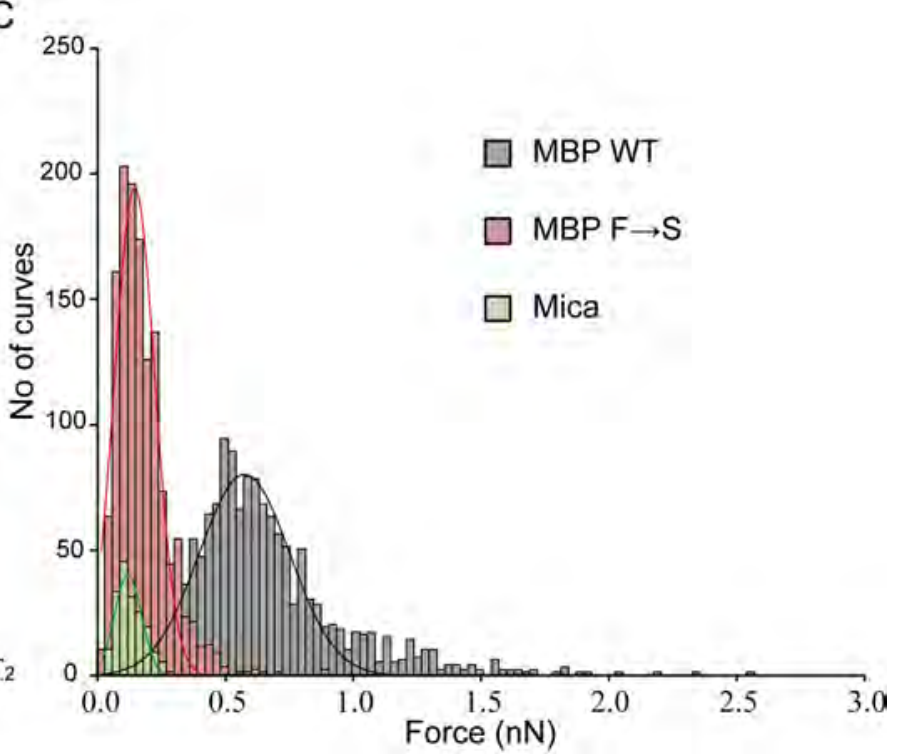

Figure 3.37: The self-interaction forces between $M B P F \rightarrow S$ mutant are smaller than wild type MBP. (A) Schematic depiction of cantilever movement during a force vs. distance measurement. Proteins (WT MBP or $\mathrm{F} \rightarrow \mathrm{S}$ mutant) were pre-adsorbed to the mica surface and the AFM tip. Shape of the curve (1) as the tip approaches the sample surface, (2) when tip touches the surface and (3) as the tip is retracted away from the sample surface. (B) Exemplary retraction curves for wild type (MBP WT) and mutant (MBP F $\rightarrow$ S) MBP. (C) Histogram of peak force measured for MBP WT (black), MBP F $\rightarrow S$ (red) and buffer (green) shows that the adhesion force between WT molecules is much higher than for the $\mathrm{F} \rightarrow \mathrm{S}$ mutant. The mean was obtained by a Gaussian fit to the histogram. Note that these experiments were performed in collaboration with P. Sánchez and Dr. I. Schaap at the Third Institute of Physics, University of Göttingen. 


\subsubsection{Functional consequences of $\mathrm{F} \rightarrow \mathrm{S}$ mutations}

To study the functional consequences of $\mathrm{F} \rightarrow \mathrm{S}$ mutations within MBP sequence, we first employed the biomimetic SLB-MBP-GUV assay. We showed before that GUVs attach to the SLBs in the presence of MBP, which is followed by MBP induced spreading of the GUVs on to the SLBs. Now, the question was whether $\mathrm{F} \rightarrow \mathrm{S}$ mutant would behave differently. Indeed, GUV spreading onto the SLBs was significantly impaired in case of $\mathrm{F} \rightarrow \mathrm{S}$ mutant (Figure 3.38 A, see Figure 3.38 B for quantifictaion). Interestingly, adhesion of GUVs to the SLBs was not affected .
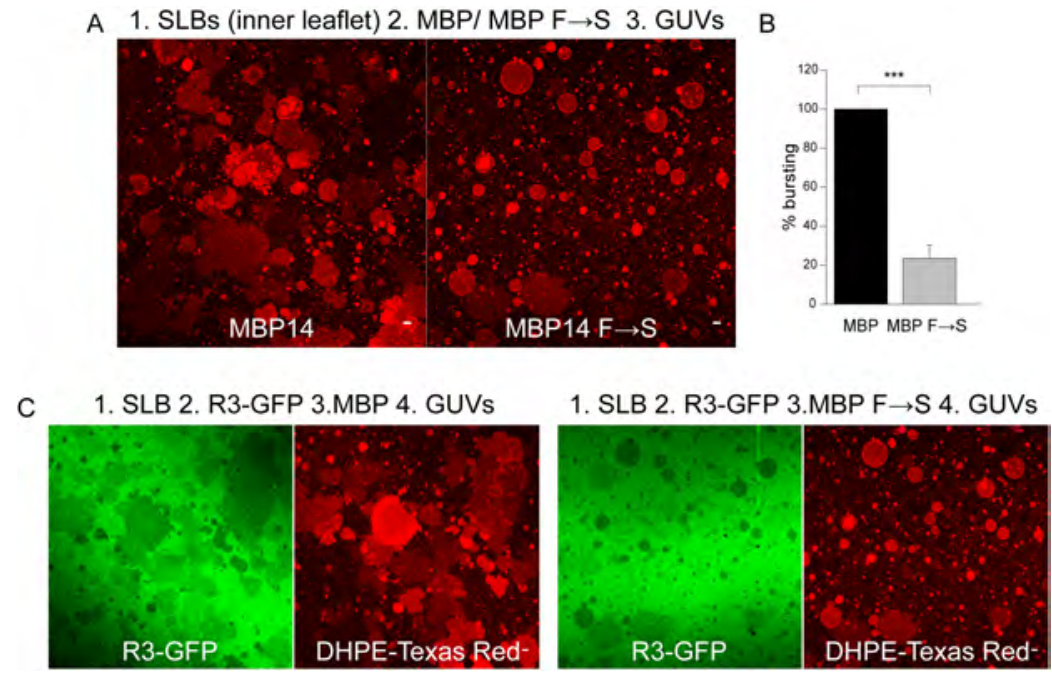

Figure 3.38: Functional consequences of $F \rightarrow S$ mutations within MBP sequence. (A) Supported lipid bilayers (SLBs) mimicking myelin inner leaflet lipid composition were prepared using mol\% of following lipids: $44 \%$ cholesterol, $27 \%$ PE, $2 \%$ PIP2, $11.5 \%$ PC, $12.5 \%$ PS, $3 \%$ SM, and $0.1 \%$ DHPE-Texas Red. $7 \mu \mathrm{M}$ of either wild type MBP (MBP14) or $\mathrm{F} \rightarrow \mathrm{S}$ mutant $(\mathrm{MBP} 14 \mathrm{~F} \rightarrow \mathrm{S})$ was added to the SLBs for $30 \mathrm{~min}$. After extensive washing, GUVs were added to the SLBs. Typical images are shown for the SLBs incubated with either MBP14 or MBP14 F $\rightarrow$ S mutant. (B) Quantification of \% GUV bursting (C) SLBs were prepared as in (A). $7 \mu \mathrm{M}$ R3-GFP was added to the SLBs followed by the addition of either MBP14 or MBP14 F $\rightarrow$ S mutant. Finally, GUVs were added from the top and extent of R3-GFP extrusion was measured. Scale bars in all the cases represent $10 \mu \mathrm{m}$. Note that all the recombinant proteins were purified in collaboration with S. Frey at Max Planck Institute for Biophysical Chemistry, Göttingen. 
Furthermore, we compared the extrusion of R3-GFP between $\mathrm{WT}$ and $\mathrm{F} \rightarrow \mathrm{S}$ mutant. As expected, the total area from which R3-GFP was extruded was significantly higher for the WT MBP in comparison to the $\mathrm{F} \rightarrow \mathrm{S}$ mutant (Figure $3.38 \mathrm{C}$ ).

In an independent approach we monitored the ability of $\mathrm{F} \rightarrow \mathrm{S}$ mutant to restore polarization in shiverer cultures. We cloned $\mathrm{F} \rightarrow \mathrm{S}$ mutant along with C-terminal HA tag into AAV2 viral vector. Next, we infected shiverer cultures with the viral particles, 2-4 hrs after plating on to the coverslips. As a positive control, we used AAV2 virus encoding wild type MBP, also carrying C-terminal HA tag. Cells were fixed at 6 DIV followed by staining for CNPase. As shown before, wild type MBP depleted CNPase from the membrane sheets (Figure 3.39 upper panel).

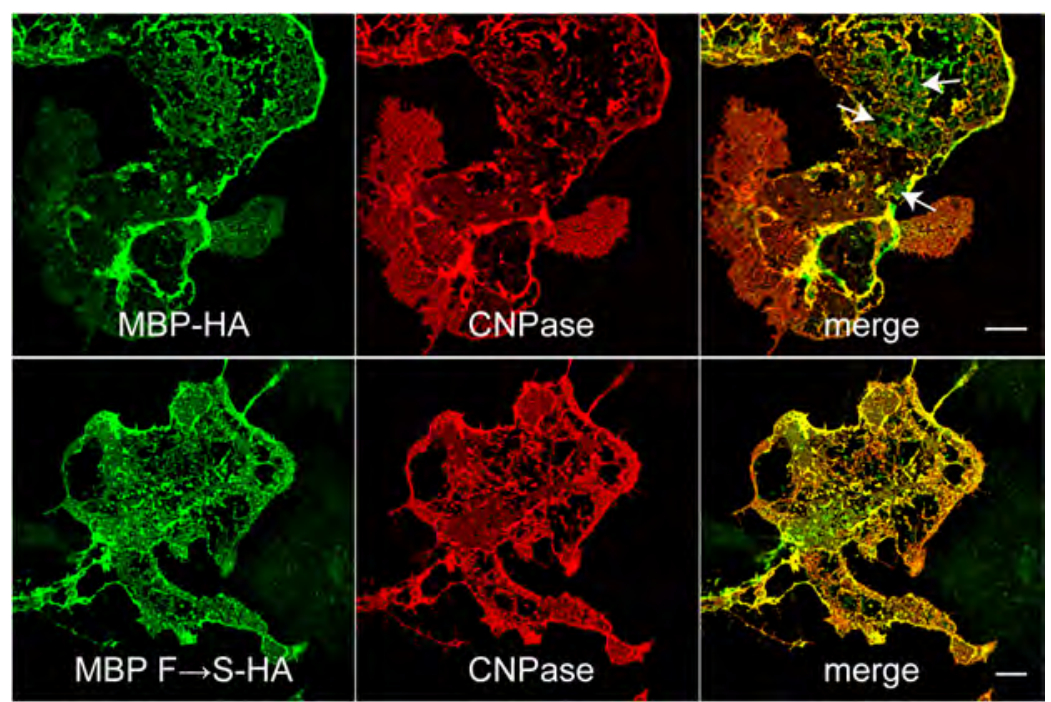

Figure 3.39: $\mathrm{F} \rightarrow \mathrm{S}$ mutant fails to rescue polarity loss in shiverer cells. Typical images for 6 DIV shiverer cells expressing wild type MBP (MBP-HA) or $F \rightarrow S$ mutant (MBP $F \rightarrow S-H A)$ and immunostained for CNPase. Expressed proteins were visualized by co-staining for the HA tags. CNPase is depleted from certain regions of the sheets with the expression of MBP-HA (upper panel, white arrows). $\mathrm{F} \rightarrow \mathrm{S}$ mutant enters into the membrane sheets of shiverer cells, but fails to extrude CNPase (lower panel). Scale bars represent $10 \mu \mathrm{m}$. Note that all the viral particles coding for $\mathrm{F} \rightarrow \mathrm{S}$ were purified in collaboration with $\mathrm{M}$. Weil at the Max Planck Institute of Experimental Medicine, Göttingen. 
Interestingly, in the absence of endogenous $\mathrm{MBP}, \mathrm{F} \rightarrow \mathrm{S}$ mutant entered the myelin membrane sheets. However, CNPase remained distributed within the sheets together with the mutant MBP (Figure 3.39 lower panel).

\subsubsection{Self-association of MBP promotes macroscopic phase separation}

The data so far clearly showed that self-association between membrane associating MBP molecules plays a crucial role in the macroscopic myelin membrane compartmentalization. The next question was whether the subsequent events of interaction between MBP molecules and lipids followed by protein oligomerization triggers phase separation.
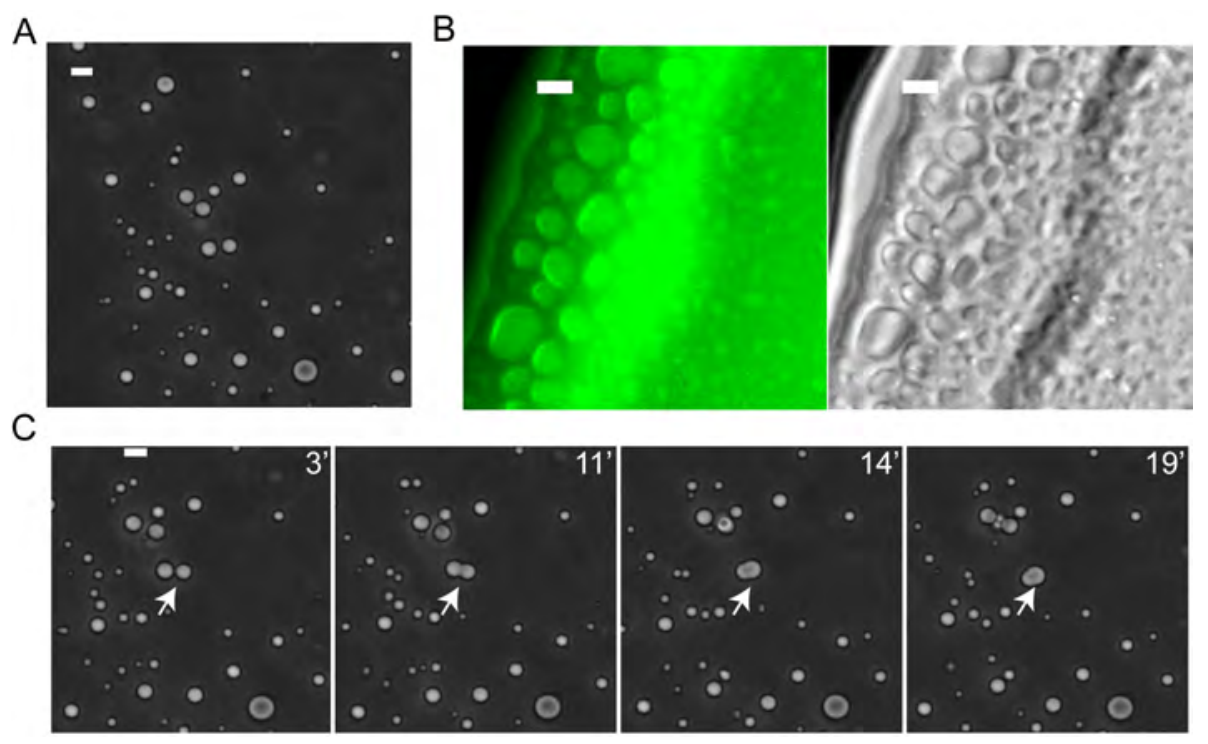

Figure 3.40: Mesoscopic phase separation of MBP in solution. (A) Liquid droplets of MBP (5 $\mathrm{mg} / \mathrm{mL}$ ) at basic $\mathrm{pH}$ visualized by phase contrast microscopy. (B) Liquid droplets of MBP labeled with Atto-488 $(5 \mathrm{mg} / \mathrm{mL})$ at basic $\mathrm{pH}$ as visualized by wide field (left panel) and phase contrast (right panel) microscopy. (C) Time-lapse images of two merging droplets. Scale bars in all cases represent $5 \mu \mathrm{m}$.

We assumed that by neutralizing the charge of MBP via interaction with lipids, oligodendrocytes exploit the potential of MBP molecules to self-associate and drive 
phase separation. If this assumption holds true, phase separation of MBP could be triggered in solution via raising $\mathrm{pH}$ close to the isoelectric point of protein. Indeed, when the concentration of MBP was raised beyond a critical limit at basic $\mathrm{pH}$, the solution became cloudy. Mixing resulted in the fragmentation of initially bicontinuous interconnected phases into $\mu \mathrm{m}$ sized droplets (Figure $3.40 \mathrm{~A}$ ). Atto-488 labelled $\mathrm{MBP}$ revealed that MBP was enriched within the droplets (Figure 3.40 B). The droplets grew and coarsen with time (Figure $3.40 \mathrm{C}$ ). Together, this behavior is consistent with a demixing phase transition in fluid. Droplets rapidly dissolved when the $\mathrm{pH}$ was neutralized showing the reversibility of the process. Importantly, when the $\mathrm{F} \rightarrow \mathrm{S}$ mutant of MBP was added into a basic solution, the solution remained clear and droplets were not observed. These results show that MBP molecules posses the inherent capability for phase separation and these physicochemical properties are likely to be physiologically and functionally relevant for the organization of myelin structure. We therefore propose that the interaction of MBP with the myelin membrane triggers a phase transition and in consequence results in protein extrusion. In conclusion, our findings provide a physico-chemical mechanism of how a protein can drive large-scale macroscopic segregations within the cytosol at the membrane interface. 
"The true sign of intelligence is not knowledge but imagination."

Albert Einstein

\section{Discussion}

One of the main functions of myelin is to insulate the axons and cluster the sodium channels at the nodes of ranvier. Due to the clustering of sodium channels, the current flows only at the nodes and not along the myelinated segments (internodes). The insulating functions of myelin arise from its truly unique molecular composition with lipids constituting more than $70 \%$ of the dry weight of myelin. In comparison, plasma membrane has equivalent ratios of lipids and proteins. Myelin composition in terms of its lipid and protein content has been the subject of extensive study. However, in spite of our burgeoning knowledge about the importance of these individual components, the key mechanisms which lead to the enrichment of lipids over proteins in myelin membrane remain obscure.

\subsection{Model system to study biogenesis of myelin membrane}

Much of our present knowledge about myelin biogenesis comes from the in vivo studies. This is because in vivo, myelin is in close contact with the axons and it is difficult to reconstitute these complex interactions in cell culture (Nave, 2010). However, upon association with the axons, individual myelin membrane domains are so tightly packed that their fine structure can only be resolved by electron microscopy. Therefore, not so surprisingly, our understanding of the cell biological mechanisms in oligodendrocytes have significantly lagged behind that of neurons. In case of neurons, a lot of insights have been acquired through in vitro dissociated cultures (Horton and Ehlers, 2003; Barnes and Polleux, 2009). Indeed, there are hints in literature that cultured oligodendrocytes can potentially serve as an 
excellent model system to study certain aspects of myelin membrane biogenesis (McCarthy and De Vellis, 1980). First, the initial events of myelination are intrinsic to oligodendrocytes that does not require association with the axons (Mirsky et al., 1980). Second, within a few days in culture, primary oligodendrocytes differentiate from bipolar to highly branched cells and subsequently to cells that extend flat membrane sheets that are enriched in myelin membrane proteins like MBP and PLP (Szuchet et al., 1986; Dubois-Dalcq et al., 1986). Third, it has been shown in our lab that even though cultured oligodendrocytes cannot recapitulate all the possible in vivo myelin subdomains, these cells can still polarize their membrane into two major domains, namely myelin membrane sheets which resemble compact myelin in composition and processes that are more similar to the non-compact areas (Dr. L. Yurlova). We employed these primary oligodendrocytes as the model system to ask the question of how compact myelin, which forms bulk of the myelin, attains its unique molecular composition. In this study, we show that it is possible to gain insights into the cell biology of oligodendrocyte polarization in relatively simple in vitro cell cultures.

\subsection{Mechanisms that regulate polarization of oligo- dendrocytes}

We found that MBP is critically involved in the process of oligodendrocyte polarization. MBP forms a physical filter and controls the diffusion of proteins into the myelin membrane sheets, based on the size of their cytosolic domains. PLP is a compact myelin protein with 11 aa on the C-terminus. However, addition of a bulky GFP restricted the protein to the processes in primary oligodendrocyte cultures (Figure 3.3). Similarly, proteins like MAG, Tmem10 and neurofascin-155 that localize to the processes or the non-compact myelin areas could gain access into the myelin membrane sheets, once their cytosolic domains (46, 100 and 90 amino acids respectively) were truncated (Figure 3.8). Serial truncations of each of these proteins revealed the cytosolic size limit to be less than 30 aa for entering into the myelin membrane sheets (Figure 3.9). Further, the polarization between compact 
and non-compact myelin was lost in the oligodendrocyte cultures prepared from MBP deficient shiverer mice. Endogenous proteins like MAG and CNPase as well as exogenously expressed proteins like PLP-YFP, CD9-GFP, CD81-GFP and Tsp2GFP were uniformly distributed throughout the cell area in the shiverer cultures (Figure 3.4). It is important to note that oligodendrocytes from shiverer mice still develop membrane sheets which shows that sheet formation is an independent event that does not require MBP. Furthermore, cytosol remains polarized in the shiverer cultures indicating that MBP regulates mainly the surface polarity in oligodendrocytes (Figure 3.6). In future it would be interesting to elucidate the molecular machinery which leads to the polarization of the cytosol in oligodendrocytes.

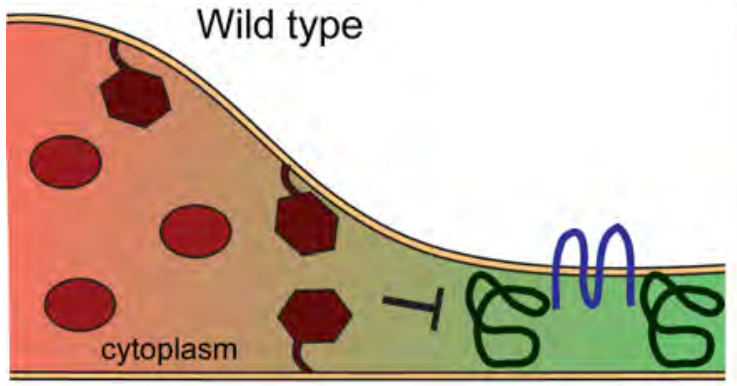

Non-compact myelin

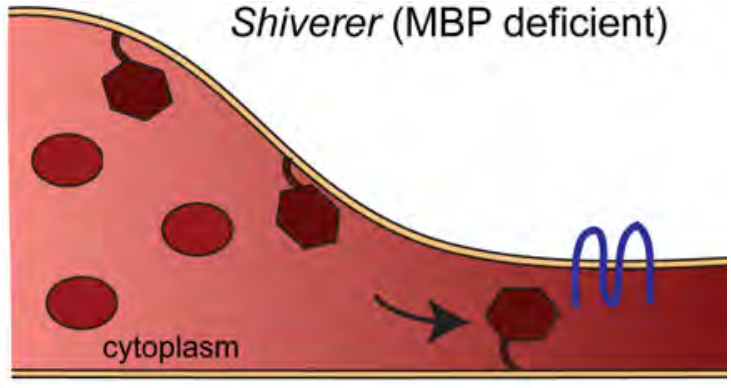

Non-compact myelin

M PLP

Figure 4.1: A diffusion barrier generates polarized myelin membrane sheets. Oligodendrocytes generate polarity at the plasma membrane by establishing a diffusion barrier. Myelin basic protein, an integral component of the compact myelin, serves as a molecular sieve and controls entry of membrane-associated proteins into the myelin membrane sheets based on the size of their cytoplasmic domain. A membrane-anchor seems to be necessary for the sorting of a cytosolic protein to membrane sheets of shiverer cells, indicating that MBP regulates polarity mainly at the plasma membrane. Modified from (Zuchero and Barres, 2011).

In addition to the in vitro cell culture experiments, we found that size barrier mechanism operates also in vivo. While CNPase normally localizes to the non-compact myelin areas (mainly inner tongue of myelin) in the wild type mice at the age of P21, it was uniformly distributed throughout the myelin sheaths in MBP deficient shiverer mice (Figure 3.15). Furthermore, we analyzed the distribution a membrane- 
anchored YFP within myelin in transgenic mice. Spassky and colleagues showed, mainly via biochemical experiments, that this construct is efficiently targeted to the myelin membrane areas (Spassky et al., 2001). However, the precise localization of the protein within the myelin domains was not investigated before. We found that this construct is specifically enriched in the non-compact myelin areas (Figure 3.14). These observations also highlight the caution required while studying the function of myelin proteins tagged with fluorescent probes.

Another important finding of our study was the role of MBP in determining the low protein-to-lipid ratios in myelin. Whereas most plasma membranes have proteinto-lipid ratio ranging between 1.0-4.0 (wt/wt), the ratio is particular low in myelin (0.25) (Singer and Nicolson, 1972). Our results show that membrane sheets contain much higher protein levels in the absence of MBP (Figure 3.13). The most likely explanation for this finding is that MBP forms a barrier that preferentially filters proteins to generate a lipid-rich membrane. Consistent with this model, myelin membrane sheets contain much higher protein levels in the absence of MBP. Thus, myelinating cells employ the MBP diffusion barrier to create the large mass of lipidrich compacted myelin membrane.

Generation and maintenance of cellular polarization has been a topic of intense investigations in a variety of cell types. It seems to follow certain common themes. For example, one way to achieve the asymmetric distribution of components is via their sorting into distinct vesicular carriers, which are in turn targeted towards different domains (Mellman and Nelson, 2008). However, this would be complicated in case of myelin as the stack of tightly packed membranes would not leave sufficient space for vesicular trafficking. Nevetheless, we cannot ignore the possibility that certain components are packed into distinct carrier vesicles and then arrive at common destination. For example, PLP has been shown to associate with cholesterol before exiting the Golgi apparatus (Simons et al., 2000). This association might lead to presorting and assembly of myelin components in the biosynthetic pathway. 
Selective retention in one of the domains is another way to generate polarized distribution of the components. When we added inert tags to the compact or non-compact myelin proteins, in each case sorting into the sheets or processes was solely decided by the size rather than any sequence per se. Still, it needs to be seen whether some proteins can gain access into the myelin membrane sheets via interaction with the fence elements. Such a mechanism has been shown for the nuclear pore complex that forms the permeability and controls the entry of protein into the nucleus based on their size. The sieve like structure has been shown to be a hydrogel made up of interlinked FG repeat domains (Frey et al., 2006). Nuclear transport receptors can overcome this size limit because of specific interactions with the inter-repeats, causing dissociation and thus transient opening of the meshwork (Frey and Görlich, 2007).

Another important mechanism for the generation of cellular polarization is through employment of diffusion barriers. In most cases, asymmetry is initially established through the polarized transport of proteins and lipids to the different domains of the membrane. Diffusion barriers are subsequently required to maintain surface asymmetry by preventing the mixing of molecules that are localized on either side of the barrier. One example is the tight junctions, which separate lateral and apical domains in epithelia (Dragsten et al., 1981). Another example includes septins which form diffusion barriers in a wide range of cellular systems and thereby cause cellular compartmentalization (Luedeke et al., 2005; Shcheprova et al., 2008; McMurray and Thorner, 2009; Kwitny et al., 2010; McMurray et al., 2011; Mostowy and Cossart, 2012). Further, diffusion barriers are also found at the axon initiation segment which separates the axon from somatodendritic compartment (Rasband, 2010a) and at the neck of a spine in the dendrites (Barral and Mansuy, 2007). In oligodendrocytes, barrier in the compacted myelin membrane is not just a fence. Because MBP molecules line the entire sheet, barrier function is intimately intertwined with the biogenesis of the polarized cell surface. As the membrane sheets do not seem to leave sufficient space for the entry of vesicular carriers, separation of membrane components must occur at the cell surface. One possibility is that oligodendrocytes extend myelin membrane sheets by lateral flow of myelin components 
within the plasma membrane. The biosynthetic trafficking may be directed to the surface of the cell body and the processes from where the diffusion of the proteins into the sheets is at least in part regulated by the size of their cytoplasmic domains. An alternative to the "lateral membrane flow" model of myelin expansion is generation of surface asymmetry via extrusion. In this model, initially molecules would mix homogeneously within the sheets. Later, heterogeneity at the surface would be achieved via MBP mediated extrusion of bulky proteins.

If the size of the cytoplasmic domain of a molecule is the main determinant for sorting into myelin, lipids should diffuse relatively freely. This is because according to picket-fence model, transmembrane proteins, when present in high density behave like pickets and restrict lipid diffusion (Nakada et al., 2003). As most of the proteins are restricted to the processes, lipid should diffuse relatively unhindered in myelin membrane sheets.

\subsection{Minimal component systems and reconstitu- tion of MBP barrier}

In this study, we also developed two independent minimal component assays for validating the function of MBP outside the myelin membrane environment. First, we established membrane based biomimetic three component assay. In this assay MBP was sandwiched between two lipid bilayers (Figure 3.17). The first bilayer was formed deposited on the coverslip and the second bilayer was added from the top in the form of giant unilamellar vesicles. We observed exclusion of membrane associated GFP from the areas where GUVs adhered and bursted onto the SLBs in the presence of MBP (Figure 3.17).

As a second assay, we established MBP barrier at the interface of the endoplasmic reticulum (ER) and plasma membrane (PM) in a fibroblast cell line (Figure 3.22). For this, we fused MBP to C-terminal domains of a number of myelin as well as non-myelin transmembrane proteins. In each case, we observed exclusion of plasma 
membrane proteins from the MBP positive areas based on the size of the cytoplasmic domains (Figure 3.24). Interestingly, similar to primary oligodendrocytes, the size limit for a protein to co-exist with MBP positive domains was less than 30 amino acids (Figure 3.25). Furthermore, the domain formation was closely dependent on the charge of the plasma membrane. Dissipation of negative charges at the plasma membrane with the addition ionomycin or sphingosine caused the retraction of MBP domains and dissolution of the barrier (Figure 3.27).

With these two assays, we could specifically look at MBP dependent effects, independent of myelin-specific factors. Furthermore, we could use either recombinant proteins or exogenous expression MBP constructs to screen for the mutants defective in self-assembly, but still capable of membrane binding.

\subsection{Self-assembling diffusion barriers}

Since diffusion barriers seem to play an important role in cellular compartmentalization, it is important to understand the nature their assembly. For example, nuclear pore complexes (NPCs) form the diffusion barrier and establish two major compartments within the eukaryotic cells, namely the nucleus and the cytoplasm (Görlich and Kutay, 1999). The structural unit of NPCs is comprised of mainly nucleoporins. A subset of these nucleoporins contain upto 50 repeat units of FG, FxFG or GLFG amino acid sequence separated by hydrophilic spacers (Denning and Rexach, 2007). These so called "FG repeat" domains are intrinsically unstructured (Denning et al., 2003) and have been shown to form elastic hydrogels that can recapitulate the selective permeability properties of the NPC (Frey et al., 2006; Frey and Görlich, 2007, 2009). Importantly, hydrogel formation seems to require hydrophobic interactions between the FG repeat domains via phenylalanine residues. Mutation of these phenylalanine residues to serines not only abolished the cross-linking between the FG repeats, but also the hydrogel formation (Frey et al., 2006). 
Strikingly, similar to nucleoporins, MBP seems to self-assemble into higher order structures upon membrane binding. Higher order assemblies of MBP were evident in the in vitro and in vivo cross-linking experiments (Figure 3.31). Furthermore, addition of MBP to the cytosolic side of two transmembrane proteins, TM10 and GypTM increased FRET efficiency by more than two-fold in PtK2 cells (Figure 3.36). How might this self-assembly work? We tested the role of hydrophobic phenylalanine residues in mediating self-interaction between the MBP molecules. For this, we mutated each of the six phenylalanine residues to hydrophilic serines. $\mathrm{F} \rightarrow \mathrm{S}$ mutant failed to establish the ER-PM domains in PtK2 cells (Figure 3.35). Similar results were obtained when phenylalanine residues were replaced with either alanine of glycine residues. However, MBP F $\rightarrow$ I mutant where phenylalanine residues were replaced with hydrophobic isoleucine residues, efficiently established ER-PM domains in PtK2 cells. These experiments revealed the need for the overall hydrophobicity at these positions. Interestingly, similar results have been obtained for the self-associating $\mathrm{A} \beta 42$ assemblies. $\mathrm{A} \beta 42$ has stretches of hydrophobic residues on the C-terminal half. Substitution of 12 hydrophobic residues with random non-polar residues in the $\mathrm{C}$-terminal half of the protein preserved the aggregation propensities (Kim and Hecht, 2006).

As described before, MBP is unstructured in solution and it is only upon binding to the membrane (specifically negatively charged lipids), this protein acquires secondary structure. We performed several independent control experiments to check the membrane binding capabilities of $\mathrm{F} \rightarrow \mathrm{S}$ mutant. MBP has been demonstrated to associate with the plasma membrane in the oligodendroglial precursor cell line, Oli-neu cells (Nawaz et al., 2009). We expressed wild type and F $\rightarrow \mathrm{S}$ MBP in Olineu cells. Both proteins showed similar association with the plasma membrane. We also purified the two variants in the bacterial expression system. Supported lipid bilayers (SLBs), mimicking inner myelin leaflet lipid composition, were incubated with $\mathrm{WT}$ and $\mathrm{F} \rightarrow \mathrm{S}$ recombinant proteins. Each protein remained bound to the SLB even after multiple washing steps. Finally, we performed liposome pull down assay with the WT and $\mathrm{F} \rightarrow \mathrm{S}$ mutant protein. The lipid composition of liposomes was similar to the inner myelin leaflet. These liposomes were incubated with similar 
concentrations of the two protein variants. We reasoned in case of association with the membranes, protein would co-pellet with liposomes upon centrifugation. Indeed, $\mathrm{F} \rightarrow \mathrm{S}$ mutant protein was almost completely depleted from the supernatant upon centrifugation (Figure 3.34).

On the molecular level, we could show that self-assembly is severely perturbed in case of $\mathrm{F} \rightarrow \mathrm{S}$ mutant. FRET assay demonstrated that the proximity between $\mathrm{F} \rightarrow \mathrm{S}$ is significantly less than the wild type molecules (Figure 3.36). In addition, we measured the adhesive forces between MBP molecules and later compared these forces for $\mathrm{F} \rightarrow \mathrm{S}$ mutant (Figure 3.37). Such measurements have been performed in the past with the wild type MBP molecules (Mueller et al., 1999), but the molecular nature of the observed long-range interaction was not clear. We now implicate hydrophobic phenylalanine residues in mediating these long range interactions between MBP molecules.

What is the functional consequence of these hydrophobic self-interactions? Interaction of MBP with large unilamellar vesicles has been investigated before (ter Beest and Hoekstra, 1993). Large unilamellar vesicles containing PS aggregated irreversibly in the presence of MBP. Furthermore, the membrane became unstable or perturbed as shown by the release of aqueous content marker, carboxyfluorescin. As mentioned before, we also saw the spreading of GUVs onto the SLBs in the presence of MBP (Figure 3.17). Importantly, mere cross-linking of the two membrane with polyvalent antibodies was not sufficient to cause the GUV bursting (Figure 3.19). Our model suggests that GUVs burst due to self-association between MBP molecules. Consistent with our model, we observed that in comparison to the wild type MBP, GUV bursting events were reduced more than five fold with the $\mathrm{F} \rightarrow \mathrm{S}$ mutant (Figure 3.38). What is the physiological relevance of this finding? One function of MBP is to extrude the bulky proteins out of the myelin membrane sheets during development (Figure 3.20). Unlike MBP, the $\mathrm{F} \rightarrow \mathrm{S}$ mutant was not able to push CNPase out of the sheets in MBP deficient shiverer cultures (Figure 3.39). Therefore, apart from the association with the inner leaflet lipids, self-interaction between MBP molecules is equally important for polarity establishment in oligoden- 
drocytes.

Apart from MBP, there are other basic proteins like MARKS that have been speculated to self-associate. For example, upon expression into macrophages, MARKS show a punctate distribution pattern (Rosen et al., 1990). Unlike MBP, in case of MARKS the basic residues are concentrated in a stretch. The effector domain of MARKS is made up of 13 aa that also contains five phenylalanine residues. When these phenylalanine residues are mutated to the alanines, the peptide still binds strongly to the acidic membrane, but with an altered mode of interaction (Andrews et al., 1999). The effector domain of MARKS with mutated phenylalanine residues is no longer able to sequester the lipid PIP2. Probably, similar to MBP, the self-association is disrupted. In future, it would be interesting to investigate the role of MBP self-association in the lateral organization of lipids within the plasma membrane. For example, MBP has been shown to sequester PIP2 in the model membranes by fluorescence quenching and electron paramagnetic resonance spectroscopy (Musse et al., 2008). Furthermore, MBP has been shown to induce the clustering of the outer leaflet lipid GalC (Fitzner et al., 2006).

Another interesting avenue for future studies would be explore whether there are other proteins within myelin that behave in a similar way as MBP. An interesting candidate is the P2 protein, which is mainly present in the PNS (Kursula, 2008). Interestingly, while the loss of MBP leads to a severe myelination defect in CNS, the effect in milder in PNS (Privat et al., 1979). Lack of severe phenotype in PNS myelin has been attributed to compensatory mechanisms by other myelin proteins like P2 and P0 (Winter, 1982). Similar to MBP, P2 is also a peripheral membrane protein. In the in vitro assays with the membranes, both MBP and P2 have been shown to induce the stacking of the bilayers (Suresh et al., 2010). Interestingly, simultaneous addition of both proteins increased the extent of stacking. As these proteins induce stacking of the membranes, the overall area occupied by the bilayer on the mica surface decreased progressively with the increasing extent of stacking. The authors speculate that MBP and P2 act in a synergistic way. Whether P2 also forms a self associating molecular sieve remains to be seen. The various minimal 
component assays developed in this study can be used to explore the role of P2. For example, it would be interesting to see whether P2 can establish ER-PM domains in PtK2 cells upon fusion to a transmembrane proteins. If this would be the case, one can test the size limit of proteins that can be excluded. Furthermore, P2 can be expressed in MBP deficient shiverer cultures and polarity re-establishment can be tested by staining for CNPase.

\subsection{Protein phase separation in solution}

Self-assembly of MBP upon association with the negatively charged lipids causes mesoscopic compact myelin domain formation. These experiments raised an intriguing question whether MBP has an intrinsic capability to phase separate from the bulk of solution. Indeed, beyond a critical concentration of MBP, charge neutralization via raising the $\mathrm{pH}$ led to the formation of macrosopic sized protein droplets. These droplets exhibited random motion due to diffusion and upon contact the two droplets merged together (Figure 3.40). The liquid like behavior of these droplets was evident from the reversibility of the process. pH neutralization lead to the rapid dissolution of the droplets. Interestingly, no droplets were observed when $\mathrm{pH}$ was raised for the $\mathrm{F} \rightarrow \mathrm{S}$ mutant.

Other examples of liquid-liquid transition includes human hemoglobin, HbA which undergoes reversible liquid-liquid separation near physiological $\mathrm{pH}$ in the presence of small amounts of polyethyleneglycol (PEG) between 35-40 $\mathrm{C}$ (Galkin et al., 2002). These liquid-liquid transitions play an important role in the formation of polymers of mutant hemoglobin, HbS in sickle cell anemia (Chen et al., 2004). Similarly, in cataract formation, opacification of the eye lens occurs due to the liquid-liquid phase separation of the gamma-crystalline proteins (Broide et al., 1991).

Our data implicates hydrophobic interactions in the formation of protein droplets. Such interactions have been proposed before to play a role in the demixing of two liquid phases. Condensed P-granules in C. elegans are amongst the physiologi- 
cally phase separated protein assemblies (Brangwynne et al., 2009). Recently, the P-granule protein, GLH1 with three tandem FG repeats was shown to readily assemble into droplets (Updike et al., 2011). Furthermore, disordered domains have been demonstrated to play an important role in the intracellular phase transitions (Kato et al., 2012). For example, many RNA-binding proteins (RBPs), enriched in low complexity sequences, were precipitated by the addition of the chemical, 5-arylisoxazole-3-carboxyamide. These RBPs form part of RNA granules. An abundant RBP, FUS (fused in sarcoma) was also precipitated with this chemical. FUS and six other candidates, when incubated at low temperature in high concentration, underwent phase transitions to form hydrogels.

Apart from hydrophobicity, mutivalency has also been identified as an important factor in promoting phase separation ( $\mathrm{Li}$ et al., 2012). In this study, many mutidomain proteins underwent phase transition in solution and formed liquid droplets. The valency of the interacting species had a direct effect on the critical concentration required for the de-mixing. For example, the interaction between purified repeats of SRC homology 3 (SH3) domain $\left(\mathrm{SH} 3_{\mathrm{n}}\right)$ and proline-rich motif (PRM) ligand $\left(\mathrm{PRM}_{\mathrm{n}}\right)$ generated droplets. Importantly, droplets formed only when the repeat number, $\mathrm{n}$ was increased beyond 3 .

Protein phase separations have been implicated in mesoscopic organization of nonmembrane bound organelles in cells. Examples include the nucleolus, the centrosomes and the germ granules (Brangwynne et al., 2009, 2011; Brangwynne, 2011; Hyman and Brangwynne, 2011; Weber and Brangwynne, 2012). Our data suggests that MBP also has intrinsic properties to separate out from the bulk solution when its charge is neutralized. However, physiologically it is the negatively charged lipids that bind and negate the electrostatic repulsions between the MBP molecules. This association between lipids and MBP in turn provides protein with a unique opportunity to de-mix from the cytoplasmic phase and organize the macroscopic, compact myelin domain within myelin. 
"The outcome of any serious research can only be to make two questions grow where only one grew before."

Thorstein Veblen

\section{Summary and Outlook}

Myelin is a unique membrane with lipids constituting more than $70 \%$ of its dry weight. By insulating axons, this membrane allows fast saltatory conduction of nerve impulses. Apart from the insulation, it also provides trophic support for the axons. The importance of myelin is underscored in de/dysmyelinating diseases like multiple sclerosis and leukodystrophies. Myelin is also an excellent example of a polarized membrane. The two major domains within myelin include the compact myelin and non-compact myelin. Compact myelin forms the bulk of myelin and is responsible for the insulating properties.

This thesis describes the molecular mechanism underlying formation of two major domains within the myelin membrane, namely the compact and non-compact domains. We used the model system of cultured oligodendrocytes that form twodimensional myelin membrane sheets. The myelin membrane sheets resembled compacted domain in composition. We found that the myelin basic protein forms a diffusion barrier on the cytoplasmic side of the myelin membrane sheets. Our results show that only proteins with cytoplasmic domains of less than 30 amino acids can cross this permeability barrier. However, in the absence of MBP, any membrane protein could gain access into the sheets, regardless of the size of the cytosolic domain. Using this cytoplasmic filtering mechanism, MBP excludes most proteins from diffusing into the myelin membrane sheets, thereby setting a pre-condition for the enrichment of lipids. Indeed lipid-to-protein ratios changed in myelin extracted from the MBP deficient shiverer mice. While MBP clearly generated asymmetry at the surface, the polarization of the cytosol was not affected in shiverer cultures. 
How the cytosol becomes polarized is an interesting question for future studies.

The presence of a diffusion barrier along the compacted myelin raised several further questions. How does MBP generate lateral asymmetry at the surface? Is MBP synthesis closely coupled to the sheet biogenesis? If sheet biogenesis is closely coupled with MBP synthesis, then bulky proteins would not enter into the sheets at any time point during development. However, we observed that sheets form prior to the expression of MBP. These unmature sheets were positive for the non-compact myelin components. With time, bulky proteins present in the unmature sheets were extruded to the non-compact domains by MBP. Therefore, surface asymmetry is achieved in oligodendrocytes via lateral unmixing of the components. This lateral unmixing was intimately dependent on the self-association between MBP molecules. Hydrophobic phenylalanine residues were found to be vital for the self-association of MBP molecules. MBP mutant in which all the phenylalanine residues were mutated to the hydrophilic serine residues, was incapable of laterally unmixing the components and generating asymmetry in shiverer cultures.

We also found that MBP possess inherent capabilities to phase separate from the bulk fluid when its basic charges are neutralized. Physiologically, electrostatic interactions between negatively charged inner leaflet lipids and basic MBP molecules probably leads to the separation of compact myelin from non-compact domains. An interesting avenue for future studies will be to address whether demyelinating events could be triggered by such phase transitions. 


\section{Bibliography}

Aggarwal, S., Yurlova, L. and Simons, M. (2011). Central nervous system myelin: structure, synthesis and assembly. Trends in Cell Biology 21, 585-93.

Ainger, K., Avossa, D., Diana, a. S., Barry, C., Barbarese, E. and Carson, J. H. (1997). Transport and localization elements in myelin basic protein mRNA. The Journal of Cell Biology 138, 1077-87.

Ainger, K., Avossa, D., Morgan, F., Hill, S. J., Barry, C., Barbarese, E. and Carson, J. H. (1993). Transport and localization of exogenous myelin basic protein mRNA microinjected into oligodendrocytes. The Journal of Cell Biology 123, 431-41.

Akowitz, A. A., Barbarese, E., Scheld, K. and Carson, J. H. (1987). Structure and Expression of Myelin Basic Protein Gene Sequences in the mld Mutant Mouse: Reiteration and Rearrangement of the Mbp Gene. Genetics 116, 447-464.

Alexander, R. T., Jaumouillé, V., Yeung, T., Furuya, W., Peltekova, I., Boucher, A., Zasloff, M., Orlowski, J. and Grinstein, S. (2011). Membrane surface charge dictates the structure and function of the epithelial $\mathrm{Na}+/ \mathrm{H}+$ exchanger. The European Molecular Biology Organization Journal 30, 679-691.

Andrews, R. K., Harris, S. J., McNally, T. and Berndt, M. C. (1999). Interactions controlling the membrane binding of basic protein domains: phenylalanine and the attachment of the myristoylated alanine-rich C-kinase substrate protein to interfaces. Biochemistry 3\%, 12527-12536.

Axelrod, D., Koppel, D. E., Schlessinger, J., Elson, E. and Webb, W. W. (1976). Mobility measurement by analysis of fluorescence photobleaching recovery kinetics. Biophysical Journal 16, 1055-1069.

Bansal, R. and Pfeiffer, S. E. (1997). FGF-2 converts mature oligodendrocytes to a novel phenotype. Journal of Neuroscience Research 50, 215-228. 
Barbarese, E., Koppel, D. E., Deutscher, M. P., Smith, C. L., Ainger, K., Morgan, F. and Carson, J. H. (1995). Protein translation components are colocalized in granules in oligodendrocytes. Journal of Cell Science 108 ( Pt 8, 2781-90.

Barnes, A. P. and Polleux, F. (2009). Establishment of axon-dendrite polarity in developing neurons. Annual Review of Neuroscience 32, 347-81.

Barral, Y. and Mansuy, I. M. (2007). Septins: cellular and functional barriers of neuronal activity. Current Biology 17, R961-3.

Becker, I., Wang-Eckhardt, L., Yaghootfam, A., Gieselmann, V. and Eckhardt, M. (2008). Differential expression of (dihydro)ceramide synthases in mouse brain: oligodendrocyte-specific expression of CerS2/Lass2. Histochemistry and Cell Biology 129, 233-41.

Ben-David, O., Pewzner-Jung, Y., Brenner, O., Laviad, E. L., Kogot-Levin, A., Weissberg, I., Biton, I. E., Pienik, R., Wang, E., Kelly, S., Alroy, J., RaasRothschild, A., Friedman, A., Brügger, B., Merrill, A. H. and Futerman, A. H. (2011). Encephalopathy caused by ablation of very long acyl chain ceramide synthesis may be largely due to reduced galactosylceramide levels. The Journal of Biological Chemistry 286, 30022-30033.

Beniac, D. R., Luckevich, M. D., Czarnota, G. J., Tompkins, T. A., Ridsdale, R. A., Ottensmeyer, F. P., Moscarello, M. A. and Harauz, G. (1997). Three-dimensional structure of myelin basic protein. I. Reconstruction via angular reconstitution of randomly oriented single particles. The Journal of Biological Chemistry 272, 4261-4268.

Bennett, V. and Lambert, S. (2000). Physiological roles of axonal ankyrins in survival of premyelinated axons and localization of voltage-gated sodium channels. Journal of Neurocytology 28, 303-18.

Bernard, C. C., Johns, T. G., Slavin, A., Ichikawa, M., Ewing, C., Liu, J. and Bettadapura, J. (1997). Myelin oligodendrocyte glycoprotein: a novel candidate autoantigen in multiple sclerosis. Journal of Molecular Medicine 75, 77-88.

Boggs, J. M. (2006). Myelin basic protein: a multifunctional protein. Cellular and Molecular Life Sciences 63, 1945-61. 
Boggs, J. M. and Moscarello, M. a. (1978). Effect of basic protein from human central nervous system myelin on lipid bilayer structure. The Journal of Membrane Biology 39, 75-96.

Boggs, J. M., Moscarello, M. A. and Papahadjopoulos, D. (1977). Phase separation of acidic and neutral phospholipids induced by human myelin basic protein. Biochemistry 16, 5420-6.

Boggs, J. M., Rangaraj, G. and Koshy, K. M. (1999). Analysis of the membraneinteracting domains of myelin basic protein by hydrophobic photolabeling. Biochimica et Biophysica Acta 1417, 254-66.

Boggs, J. M., Yip, P. M., Rangaraj, G. and Jo, E. (1997). Effect of posttranslational modifications to myelin basic protein on its ability to aggregate acidic lipid vesicles. Biochemistry 36, 5065-71.

Boison, D., Büssow, H., D’Urso, D., Müller, H. W. and Stoffel, W. (1995). Adhesive properties of proteolipid protein are responsible for the compaction of CNS myelin sheaths. The Journal of Neuroscience 15, 5502-5513.

Boison, D. and Stoffel, W. (1994). Disruption of the compacted myelin sheath of axons of the central nervous system in proteolipid protein-deficient mice. Proceedings of the National Academy of Sciences 91, 11709-11713.

Bradford, M. M. (1976). A rapid and sensitive method for the quantitation of microgram quantities of protein utilizing the principle of protein-dye binding. Analytical Biochemistry 72, 248-54.

Brangwynne, C. P. (2011). Soft active aggregates: mechanics, dynamics and selfassembly of liquid-like intracellular protein bodies. Soft Matter 7, 3052.

Brangwynne, C. P., Eckmann, C. R., Courson, D. S., Rybarska, A., Hoege, C., Gharakhani, J., Jülicher, F. and Hyman, A. a. (2009). Germline P granules are liquid droplets that localize by controlled dissolution/condensation. Science 324, $1729-32$.

Brangwynne, C. P., Mitchison, T. J. and Hyman, A. a. (2011). Active liquid-like behavior of nucleoli determines their size and shape in Xenopus laevis oocytes. Proceedings of the National Academy of Sciences 108, 4334-9. 
Broide, M. L., Berland, C. R., Pande, J., Ogun, O. O. and Benedek, G. B. (1991). Binary-liquid phase separation of lens protein solutions. Proceedings of the National Academy of Sciences of the United States of America 88, 5660-4.

Bronstein, J. M., Micevych, P. E. and Chen, K. (1997). Oligodendrocyte-specific protein (OSP) is a major component of CNS myelin. Journal of Neuroscience Research 50, 713-720.

Bronstein, J. M., Popper, P., Micevych, P. E. and Farber, D. B. (1996). Isolation and characterization of a novel oligodendrocyte-specific protein. Neurology 47 , $772-778$.

Bryant, D. M. and Mostov, K. E. (2008). From cells to organs: building polarized tissue. Nature Reviews. Molecular Cell Biology 9, 887-901.

Carson, J. H., Worboys, K., Ainger, K. and Barbarese, E. (1997). Translocation of myelin basic protein mRNA in oligodendrocytes requires microtubules and kinesin. Cell Motility and the Cytoskeleton 38, 318-28.

Caudron, F. and Barral, Y. (2009). Septins and the lateral compartmentalization of eukaryotic membranes. Developmental Cell 16, 493-506.

Charles, P., Tait, S., Faivre-Sarrailh, C., Barbin, G., Gunn-Moore, F., DenisenkoNehrbass, N., Guennoc, A.-M., Girault, J.-A., Brophy, P. J. and Lubetzki, C. (2002). Neurofascin is a glial receptor for the paranodin/Caspr-contactin axonal complex at the axoglial junction. Current Biology 12, 217-220.

Chen, Q., Vekilov, P. G., Nagel, R. L. and Hirsch, R. E. (2004). Liquid-liquid phase separation in hemoglobins: distinct aggregation mechanisms of the beta6 mutants. Biophysical Journal 86, 1702-12.

Chernoff, G. F. (1981). Shiverer: an autosomal recessive mutant mouse with myelin deficiency. The Journal of Heredity 72, 128.

Coetzee, T., Fujita, N., Dupree, J., Shi, R., Blight, A., Suzuki, K. and Popko, B. (1996). Myelination in the absence of galactocerebroside and sulfatide: normal structure with abnormal function and regional instability. Cell 86, 209-219.

Colman, D. R., Kreibich, G., Frey, a. B. and Sabatini, D. D. (1982). Synthesis and incorporation of myelin polypeptides into CNS myelin. The Journal of Cell Biology 95, 598-608. 
Cove, D. J. (2000). The generation and modification of cell polarity. Journal of Experimental Botany 51, 831-8.

Denning, D. P., Patel, S. S., Uversky, V., Fink, A. L. and Rexach, M. (2003). Disorder in the nuclear pore complex: the FG repeat regions of nucleoporins are natively unfolded. Proceedings of the National Academy of Sciences 100, 24502455.

Denning, D. P. and Rexach, M. F. (2007). Rapid evolution exposes the boundaries of domain structure and function in natively unfolded FG nucleoporins. Molecular Cellular Proteomics 6, 272-282.

Devaux, J. and Gow, A. (2008). Tight junctions potentiate the insulative properties of small CNS myelinated axons. The Journal of Cell Biology 183, 909-921.

Dhaunchak, A.-S. and Nave, K.-A. (2007). A common mechanism of PLP/DM20 misfolding causes cysteine-mediated endoplasmic reticulum retention in oligodendrocytes and Pelizaeus-Merzbacher disease. Proceedings of the National Academy of Sciences 104, 17813-17818.

Dragsten, P. R., Blumenthal, R. and Handler, J. S. (1981). Membrane asymmetry in epithelia: is the tight junction a barrier to diffusion in the plasma membrane? Nature 294, 718-722.

Dubois-Dalcq, M., Behar, T., Hudson, L. and Lazzarini, R. A. (1986). Emergence of three myelin proteins in oligodendrocytes cultured without neurons. The Journal of Cell Biology 102, 384-392.

Dunker, A., Brown, C., Lawson, J. and Lm (2002). Intrinsic Disorder and Protein Function. Biochemistry 41, 6573-6582.

Eggeling, C., Ringemann, C., Medda, R., Schwarzmann, G., Sandhoff, K., Polyakova, S., Belov, V. N., Hein, B., Von Middendorff, C., Schönle, A. and Hell, S. W. (2009). Direct observation of the nanoscale dynamics of membrane lipids in a living cell. Nature 457, 1159-62.

Eisele, N. B., Frey, S., Piehler, J., Görlich, D. and Richter, R. P. (2010). Ultrathin nucleoporin phenylalanine-glycine repeat films and their interaction with nuclear transport receptors. EMBO Reports 11, 366-372. 
Emery, B. (2010). Regulation of oligodendrocyte differentiation and myelination. Science 330, 779-82.

Ercan, E., Momburg, F., Engel, U., Temmerman, K., Nickel, W. and Seedorf, M. (2009). A conserved, lipid-mediated sorting mechanism of yeast Ist2 and mammalian STIM proteins to the peripheral ER. Traffic 10, 1802-1818.

Fairbanks, G., Steck, T. L. and Wallach, D. F. (1971). Electrophoretic analysis of the major polypeptides of the human erythrocyte membrane. Biochemistry 10 , 2606-2617.

Fink, A. L. (2005). Natively unfolded proteins. Current Opinion in Structural Biology 15, 35-41.

Fitzner, D., Schneider, A., Kippert, A., Möbius, W., Willig, K. I., Hell, S. W., Bunt, G., Gaus, K. and Simons, M. (2006). Myelin basic protein-dependent plasma membrane reorganization in the formation of myelin. The European Molecular Biology Organization Journal 25, 5037-48.

Fogel, A. I., Stagi, M., Perez De Arce, K. and Biederer, T. (2011). Lateral assembly of the immunoglobulin protein SynCAM 1 controls its adhesive function and instructs synapse formation. The European Molecular Biology Organization Journal 30, 4728-38.

Folch, J. and Lees, M. (1951). Proteolipides, a new type of tissue lipoproteins; their isolation from brain. The Journal of Biological Chemistry 191, 807-17.

Folch, J., Lees, M. and Stanley, G. H. S. (1957). A simple method for the isolation and purification of total lipids from animal tissues. The Journal of Biological Chemistry 226, 497-509.

Fölsch, H. (2008). Regulation of membrane trafficking in polarized epithelial cells. Current Opinion in Cell Biology 20, 208-13.

Fölsch, H., Ohno, H., Bonifacino, J. S. and Mellman, I. (1999). A novel clathrin adaptor complex mediates basolateral targeting in polarized epithelial cells. Cell 99, 189-98.

Francone, V. P., Maggipinto, M. J., Kosturko, L. D. and Barbarese, E. (2007). The microtubule-associated protein tumor overexpressed gene/cytoskeleton-associated 
protein 5 is necessary for myelin basic protein expression in oligodendrocytes. The Journal of Neuroscience 27, 7654-62.

Frank, M., Atanasoski, S., Sancho, S., Magyar, J. P., Rülicke, T., Schwab, M. E. and Suter, U. (2000). Progressive segregation of unmyelinated axons in peripheral nerves, myelin alterations in the CNS, and cyst formation in the kidneys of myelin and lymphocyte protein-overexpressing mice. Journal of Neurochemistry 75, 1927-1939.

Frey, S. and Görlich, D. (2007). A saturated FG-repeat hydrogel can reproduce the permeability properties of nuclear pore complexes. Cell 130, 512-23.

Frey, S. and Görlich, D. (2009). FG/FxFG as well as GLFG repeats form a selective permeability barrier with self-healing properties. The European Molecular Biology Organization Journal 28, 2554-67.

Frey, S., Richter, R. P. and Görlich, D. (2006). FG-rich repeats of nuclear pore proteins form a three-dimensional meshwork with hydrogel-like properties. Science $314,815-7$.

Friedrichson, T. and Kurzchalia, T. V. (1998). Microdomains of GPI-anchored proteins in living cells revealed by crosslinking. Nature 394, 802-805.

Galiano, M., Jha, S., Ho, T.-Y., Zhang, C., Ogawa, Y., Chang, K.-J., Stankewich, M., Mohler, P. and Rasband, M. (2012). A Distal Axonal Cytoskeleton Forms an Intra-Axonal Boundary that Controls Axon Initial Segment Assembly. Cell 149, $1125-1139$.

Galkin, O., Chen, K., Nagel, R. L., Hirsch, R. E. and Vekilov, P. G. (2002). Liquidliquid separation in solutions of normal and sickle cell hemoglobin. Proceedings of the National Academy of Sciences 99, 8479-83.

García-Sáez, A. J., Carrer, D. C. and Schwille, P. (2010). Fluorescence correlation spectroscopy for the study of membrane dynamics and organization in giant unilamellar vesicles. Methods In Molecular Biology 606, 493-508.

Garratt, A. N., Voiculescu, O., Topilko, P., Charnay, P. and Birchmeier, C. (2000). A Dual Role of erbB2 in Myelination and in Expansion of the Schwann Cell Precursor Pool. The Journal of Cell Biology 148, 1035-1046. 
Golan, N., Adamsky, K., Kartvelishvily, E., Brockschnieder, D., Möbius, W., Spiegel, I., Roth, A. D., Thomson, C. E., Rechavi, G. and Peles, E. (2008). Identification of Tmem10/Opalin as an oligodendrocyte enriched gene using expression profiling combined with genetic cell ablation. Glia 56, 1176-1186.

Gollan, L., Salomon, D., Salzer, J. L. and Peles, E. (2003). Caspr regulates the processing of contactin and inhibits its binding to neurofascin. The Journal of Cell Biology 163, 1213-1218.

Gomez, C. M., Muggleton-Harris, A. L., Whittingham, D. G., Hood, L. E. and Readhead, C. (1990). Rapid preimplantation detection of mutant (shiverer) and normal alleles of the mouse myelin basic protein gene allowing selective implantation and birth of live young. Proceedings of the National Academy of Sciences 87, 4481-4484.

Görlich, D. and Kutay, U. (1999). Transport between the cell nucleus and the cytoplasm. Annual Review of Cell and Developmental Biology 15, 607-660.

Gow, A., Southwood, C. M., Li, J. S., Pariali, M., Riordan, G. P., Brodie, S. E., Danias, J., Bronstein, J. M., Kachar, B. and Lazzarini, R. A. (1999). CNS myelin and sertoli cell tight junction strands are absent in Osp/claudin-11 null mice. Cell 99, 649-659.

Griffiths, I., Klugmann, M., Anderson, T., Yool, D., Thomson, C., Schwab, M. H., Schneider, A., Zimmermann, F., McCulloch, M., Nadon, N. and Nave, K. A. (1998). Axonal swellings and degeneration in mice lacking the major proteolipid of myelin. Science 280, 1610-1613.

Harauz, G., Ishiyama, N., Hill, C. M. D., Bates, I. R., Libich, D. S. and Farès, C. (2004). Myelin basic protein-diverse conformational states of an intrinsically unstructured protein and its roles in myelin assembly and multiple sclerosis. Micron 35, 503-42.

Harauz, G., Ladizhansky, V. and Boggs, J. M. (2009). Structural polymorphism and multifunctionality of myelin basic protein. Biochemistry 48, 8094-104.

Hedstrom, K. L., Ogawa, Y. and Rasband, M. N. (2008). AnkyrinG is required for maintenance of the axon initial segment and neuronal polarity. The Journal of Cell Biology 183, 635-40. 
Hill, C. M., Bates, I. R., White, G. F., Hallett, F. R. and Harauz, G. (2002). Effects of the osmolyte trimethylamine-N-oxide on conformation, self-association, and two-dimensional crystallization of myelin basic protein. Journal of Structural Biology 139, 13-26.

Hill, C. M. D., Haines, J. D., Antler, C. E., Bates, I. R., Libich, D. S. and Harauz, G. (2003). Terminal deletion mutants of myelin basic protein: new insights into self-association and phospholipid interactions. Micron 34, 25-37.

Hoek, K. S., Kidd, G. J., Carson, J. H. and Smith, R. (1998). hnRNP A2 selectively binds the cytoplasmic transport sequence of myelin basic protein mRNA. Biochemistry 37, 7021-9.

Horton, A. C. and Ehlers, M. D. (2003). Neuronal polarity and trafficking. Neuron 40, 277-95.

Hoshi, T., Suzuki, A., Hayashi, S., Tohyama, K., Hayashi, A., Yamaguchi, Y., Takeuchi, K. and Baba, H. (2007). Nodal protrusions, increased SchmidtLanterman incisures, and paranodal disorganization are characteristic features of sulfatide-deficient peripheral nerves. Glia 55, 584-594.

Hu, Q., Milenkovic, L., Jin, H., Scott, M. P., Nachury, M. V., Spiliotis, E. T. and Nelson, W. J. (2010). A septin diffusion barrier at the base of the primary cilium maintains ciliary membrane protein distribution. Science 329, 436-9.

Hu, Y., Doudevski, I., Wood, D., Moscarello, M., Husted, C., Genain, C., Zasadzinski, J. A. and Israelachvili, J. (2004). Synergistic interactions of lipids and myelin basic protein. Proceedings of the National Academy of Sciences 101, 13466-13471.

$\mathrm{Hu}, \mathrm{Y}$. and Israelachvili, J. (2008). Lateral reorganization of myelin lipid domains by myelin basic protein studied at the air-water interface. Colloids and Surfaces B Biointerfaces 62, 22-30.

Humbert, P. O., Dow, L. E. and Russell, S. M. (2006). The Scribble and Par complexes in polarity and migration: friends or foes? Trends in Cell Biology 16, 622-630.

Hunziker, W. and Fumey, C. (1994). A di-leucine motif mediates endocytosis and basolateral sorting of macrophage IgG Fc receptors in MDCK cells. The European Molecular Biology Organization Journal 13, 2963-9. 
Hutterer, A., Betschinger, J., Petronczki, M. and Knoblich, J. A. (2004). Sequential Roles of Cdc42, Par-6, aPKC , and Lgl in the Establishment of Epithelial Polarity during Drosophila Embryogenesis. Cell 6, 845-854.

Hyman, A. A. and Brangwynne, C. P. (2011). Forum Beyond Stereospecificity : Liquids and Mesoscale Organization of Cytoplasm. Developmental Cell 21, 1416.

Imgrund, S., Hartmann, D., Farwanah, H., Eckhardt, M., Sandhoff, R., Degen, J., Gieselmann, V., Sandhoff, K. and Willecke, K. (2009). Adult ceramide synthase 2 (CERS2)-deficient mice exhibit myelin sheath defects, cerebellar degeneration, and hepatocarcinomas. The Journal of Biological Chemistry 284, 33549-60.

Inouye, H. and Kirschner, D. A. (1988). Membrane interactions in nerve myelin: II. Determination of surface charge from biochemical data. Biophysical Journal 53, $247-260$.

Jahn, O., Tenzer, S. and Werner, H. B. (2009). Myelin Proteomics: Molecular Anatomy of an Insulating Sheath. Molecular Neurobiology 40, 55-72.

Jo, E. and Boggs, J. M. (1995). Aggregation of acidic lipid vesicles by myelin basic protein: dependence on potassium concentration. Biochemistry 34, 13705-16.

Jung, M., Krämer, E., Grzenkowski, M., Tang, K., Blakemore, W., Aguzzi, A., Khazaie, K., Chlichlia, K., Von Blankenfeld, G. and Kettenmann, H. (1995). Lines of murine oligodendroglial precursor cells immortalized by an activated neu tyrosine kinase show distinct degrees of interaction with axons in vitro and in vivo. European Journal of Neuroscience 7, 1245-1265.

Kahya, N., Brown, D. A. and Schwille, P. (2005). Raft partitioning and dynamic behavior of human placental alkaline phosphatase in giant unilamellar vesicles. Biochemistry 44, 7479-7489.

Kamholz, J., Toffenetti, J. and Lazzarini, R. A. (1988). Organization and expression of the human myelin basic protein gene. Journal of Neuroscience Research 21, $62-70$.

Kato, M., Han, T. W., Xie, S., Shi, K., Du, X., Wu, L. C., Mirzaei, H., Goldsmith, E. J., Longgood, J., Pei, J., Grishin, N. V., Frantz, D. E., Schneider, J. W., Chen, S., Li, L., Sawaya, M. R., Eisenberg, D., Tycko, R. and McKnight, S. L. 
(2012). Cell-free formation of RNA granules: low complexity sequence domains form dynamic fibers within hydrogels. Cell 149, 753-67.

Katsuki, M., Sato, M., Kimura, M., Yokoyama, M., Kobayashi, K. and Nomura, T. (1988). Conversion of normal behavior to shiverer by myelin basic protein antisense cDNA in transgenic mice. Science 241, 593-595.

Keniry, M. A. and Smith, R. (1979). Circular dichroic analysis of the secondary structure of myelin basic protein and derived peptides bound to detergents and to lipid vesicles. Biochimica et Biophysica Acta 578, 381-391.

Kim, W. and Hecht, M. H. (2006). Generic hydrophobic residues are sufficient to promote aggregation of the Alzheimer's A $\beta 42$ peptide. Proceedings of the National Academy of Sciences 103, 15824-15829.

Kimura, M., Sato, M., Akatsuka, A., Nozawa-Kimura, S., Takahashi, R., Yokoyama, M., Nomura, T. and Katsuki, M. (1989). Restoration of myelin formation by a single type of myelin basic protein in transgenic shiverer mice. Proceedings of the National Academy of Sciences 86, 5661-5665.

Kippert, A., Trajkovic, K., Fitzner, D., Opitz, L. and Simons, M. (2008). Identification of Tmem10/Opalin as a novel marker for oligodendrocytes using gene expression profiling. BMC Neuroscience 9, 40.

Klein, D. E., Lee, A., Frank, D. W., Marks, M. S. and Lemmon, M. A. (1998). The pleckstrin homology domains of dynamin isoforms require oligomerization for high affinity phosphoinositide binding. The Journal of Biological Chemistry 273, 27725-27733.

Kosturko, L. D., Maggipinto, M. J., Korza, G., Lee, J. W., Carson, J. H. and Barbarese, E. (2006). Heterogeneous Nuclear Ribonucleoprotein (hnRNP) E1 Binds to hnRNP A2 and Inhibits Translation of A2 Response Element mRNAs. Molecular Biology of the Cell 17, 3521-3533.

Kristensson, K., Zeller, N. K., Dubois-Dalcq, M. E. and Lazzarini, R. A. (1986). Expression of myelin basic protein gene in the developing rat brain as revealed by in situ hybridization. The Journal of Histochemistry and Cytochemistry 34, 467-473.

Kursula, P. (2008). Structural properties of proteins specific to the myelin sheath. Amino acids 34, 175-85. 
Kwitny, S., Klaus, A. V. and Hunnicutt, G. R. (2010). The annulus of the mouse sperm tail is required to establish a membrane diffusion barrier that is engaged during the late steps of spermiogenesis. Biology of Reproduction 82, 669-78.

Laemmli, U. K. (1970). Cleavage of structural proteins during the assembly of the head of bacteriophage T4. Nature 227, 680-685.

Lappe-Siefke, C., Goebbels, S., Gravel, M., Nicksch, E., Lee, J., Braun, P. E., Griffiths, I. R. and Nave, K.-A. (2003). Disruption of Cnp1 uncouples oligodendroglial functions in axonal support and myelination. Nature Genetics 33, 366-374.

Larocca, J. N. and Norton, W. T. (2007). Isolation of myelin. Current Protocols in Cell Biology Chapter 3, Unit3.25.

Laursen, L. S., Chan, C. W. and Ffrench-Constant, C. (2011). Translation of myelin basic protein mRNA in oligodendrocytes is regulated by integrin activation and hnRNP-K. The Journal of Cell Biology 192, 797-811.

Lemmon, M. A., Flanagan, J. M., Hunt, J. F., Adair, B. D., Bormann, B. J., Dempsey, C. E. and Engelman, D. M. (1992). Glycophorin A dimerization is driven by specific interactions between transmembrane alpha-helices. The Journal of Biological Chemistry 267, 7683-7689.

Li, C., Tropak, M. B., Gerlai, R., Clapoff, S., Abramow-Newerly, W., Trapp, B., Peterson, A. and Roder, J. (1994). Myelination in the absence of myelin-associated glycoprotein. Nature 369, 747-750.

Li, P., Banjade, S., Cheng, H.-C., Kim, S., Chen, B., Guo, L., Llaguno, M., Hollingsworth, J. V., King, D. S., Banani, S. F., Russo, P. S., Jiang, Q.-X., Nixon, B. T. and Rosen, M. K. (2012). Phase transitions in the assembly of multivalent signalling proteins. Nature 483, 336-340.

Lu, Q. R., Sun, T., Zhu, Z., Ma, N., Garcia, M., Stiles, C. D. and Rowitch, D. H. (2002). Common developmental requirement for Olig function indicates a motor neuron/oligodendrocyte connection. Cell 109, 75-86.

Luedeke, C., Frei, S. B., Sbalzarini, I., Schwarz, H., Spang, A. and Barral, Y. (2005). Septin-dependent compartmentalization of the endoplasmic reticulum during yeast polarized growth. The Journal of Cell Biology 169, 897-908. 
Macklin, W. B., Campagnoni, C. W., Deininger, P. L. and Gardinier, M. V. (1987). Structure and expression of the mouse myelin proteolipid protein gene. Journal of Neuroscience Research 18, 383-394.

Marcus, J., Honigbaum, S., Shroff, S., Honke, K., Rosenbluth, J. and Dupree, J. L. (2006). Sulfatide is essential for the maintenance of CNS myelin and axon structure. Glia 53, 372-381.

Marsh, D., Horváth, L. I., Swamy, M. J., Mantripragada, S. and Kleinschmidt, J. H. (2002). Interaction of membrane-spanning proteins with peripheral and lipid-anchored membrane proteins: perspectives from protein-lipid interactions (Review). Molecular Membrane Biology 19, 247-55.

Maurel, P., Einheber, S., Galinska, J., Thaker, P., Lam, I., Rubin, M. B., Scherer, S. S., Murakami, Y., Gutmann, D. H. and Salzer, J. L. (2007). Nectin-like proteins mediate axon Schwann cell interactions along the internode and are essential for myelination. The Journal of Cell Biology 178, 861-874.

McCarthy, K. D. and De Vellis, J. (1980). Preparation of separate astroglial and oligodendroglial cell cultures from rat cerebral tissue. The Journal of Cell Biology 85, 890-902.

McKinnon, R. D., Matsui, T., Aranda, M. and Dubois-Dalcq, M. (1991). A role for fibroblast growth factor in oligodendrocyte development. Annals Of The New York Academy Of Sciences 638, 378-386.

McLaughlin, S., Wang, J., Gambhir, A. and Murray, D. (2002). PIP(2) and proteins: interactions, organization, and information flow. Annual Review of Biophysics and Biomolecular Structure 31, 151-175.

McMurray, M. a., Bertin, A., Garcia, G., Lam, L., Nogales, E. and Thorner, J. (2011). Septin filament formation is essential in budding yeast. Developmental Cell 20, 540-9.

McMurray, M. a. and Thorner, J. (2009). Septins: molecular partitioning and the generation of cellular asymmetry. Cell Division 4, 18.

Mellman, I. and Nelson, W. J. (2008). Coordinated protein sorting, targeting and distribution in polarized cells. Nature Reviews. Molecular Cell Biology 9, 833-45. 
Michailov, G. V., Sereda, M. W., Brinkmann, B. G., Fischer, T. M., Haug, B., Birchmeier, C., Role, L., Lai, C., Schwab, M. H. and Nave, K.-A. (2004). Axonal neuregulin-1 regulates myelin sheath thickness. Science 304, 700-703.

Min, Y., Kristiansen, K., Boggs, J. M., Husted, C., Zasadzinski, J. a. and Israelachvili, J. (2009). Interaction forces and adhesion of supported myelin lipid bilayers modulated by myelin basic protein. Proceedings of the National Academy of Sciences 106, 3154-9.

Mirsky, R., Winter, J., Abney, E. R., Pruss, R. M., Gavrilovic, J. and Raff, M. C. (1980). Myelin-specific proteins and glycolipids in rat Schwann cells and oligodendrocytes in culture. The Journal of Cell Biology 84, 483-494.

Möbius, W., Patzig, J., Nave, K.-A. and Werner, H. B. (2008). Phylogeny of proteolipid proteins: divergence, constraints, and the evolution of novel functions in myelination and neuroprotection. Neuron Glia Biology 4, 111-127.

Mohler, P. J., Gramolini, A. O. and Bennett, V. (2002). Ankyrins. Journal of Cell Science 115, 1565-6.

Mostowy, S. and Cossart, P. (2012). Septins: the fourth component of the cytoskeleton. Nature Reviews. Molecular Cell Biology 13, 183-94.

Mueller, H., Butt, H. J. and Bamberg, E. (1999). Force measurements on myelin basic protein adsorbed to mica and lipid bilayer surfaces done with the atomic force microscope. Biophysical Journal 76, 1072-1079.

Munro, T. P., Magee, R. J., Kidd, G. J., Carson, J. H., Barbarese, E., Smith, L. M. and Smith, R. (2006). Mutational analysis of a heterogeneous nuclear ribonucleoprotein A2 response element for RNA trafficking. The Journal of Biological Chemistry 281, 2478-2488.

Murray, D., Ben-Tal, N., Honig, B. and McLaughlin, S. (1997). Electrostatic interaction of myristoylated proteins with membranes: simple physics, complicated biology. Structure London England 1993 5, 985-989.

Musse, A. a., Boggs, J. M. and Harauz, G. (2006). Deimination of membrane-bound myelin basic protein in multiple sclerosis exposes an immunodominant epitope. Proceedings of the National Academy of Sciences 103, 4422-7. 
Musse, A. a., Gao, W., Homchaudhuri, L., Boggs, J. M. and Harauz, G. (2008). Myelin basic protein as a "PI(4,5)P2-modulin": a new biological function for a major central nervous system protein. Biochemistry 47, 10372-82.

Nakada, C., Ritchie, K., Oba, Y., Nakamura, M., Hotta, Y., Iino, R., Kasai, R. S., Yamaguchi, K., Fujiwara, T. and Kusumi, A. (2003). Accumulation of anchored proteins forms membrane diffusion barriers during neuronal polarization. Nature Cell Biology 5, 626-32.

Nave, K.-A. (2010). Myelination and the trophic support of long axons. Nature Reviews. Neuroscience 11, 275-83.

Nave, K. A., Lai, C., Bloom, F. E. and Milner, R. J. (1987). Splice site selection in the proteolipid protein (PLP) gene transcript and primary structure of the DM-20 protein of central nervous system myelin. Proceedings of the National Academy of Sciences 84, 5665-5669.

Nawaz, S., Kippert, A., Saab, a. S., Werner, H. B., Lang, T., Nave, K.-a. and Simons, M. (2009). Phosphatidylinositol 4,5-Bisphosphate-Dependent Interaction of Myelin Basic Protein with the Plasma Membrane in Oligodendroglial Cells and Its Rapid Perturbation by Elevated Calcium. The Journal of Neuroscience 29, 4794-4807.

Ng, D. P. and Deber, C. M. (2010). Modulation of the oligomerization of myelin proteolipid protein by transmembrane helix interaction motifs. Biochemistry 49, 6896-6902.

Noble, M., Murray, K., Stroobant, P., Waterfield, M. D. and Riddle, P. (1988). Platelet-derived growth factor promotes division and motility and inhibits premature differentiation of the oligodendrocyte/type- 2 astrocyte progenitor cell. Nature 333, 560-562.

Norton, W. T. and Autilio, L. A. (1965). THE CHEMICAL COMPOSITION OF BOVINE CNS MYELIN. Annals Of The New York Academy Of Sciences 122, $77-85$.

Norton, W. T. and Poduslo, S. E. (1973). Myelination in rat brain: method of myelin isolation. Journal of Neurochemistry 21, 749-757.

Okano, H., Miura, M., Moriguchi, A., Ikenaka, K., Tsukada, Y. and Mikoshiba, K. (1987). Inefficient transcription of the myelin basic protein gene possibly causes 
hypomyelination in myelin-deficient mutant mice. Journal of Neurochemistry 48 , $470-6$.

Ozçelik, M., Cotter, L., Jacob, C., Pereira, J. A., Relvas, J. a. B., Suter, U. and Tricaud, N. (2010). Pals1 is a major regulator of the epithelial-like polarization and the extension of the myelin sheath in peripheral nerves. The Journal of Neuroscience 30, 4120-4131.

Pedraza, L. (1997). Nuclear transport of myelin basic protein. Journal of Neuroscience Research 50, 258-264.

Pedraza, L., Huang, J. K. and Colman, D. R. (2001). Organizing principles of the axoglial apparatus. Neuron 30, 335-344.

Pereira, J. A., Lebrun-Julien, F. and Suter, U. (2011). Molecular mechanisms regulating myelination in the peripheral nervous system. Trends in Neurosciences 35, $123-34$.

Pfeiffer, S. E., Warrington, A. E. and Bansal, R. (1993). The oligodendrocyte and its many cellular processes. Trends in Cell Biology 3, 191-197.

Pham-Dinh, D., Mattei, M. G., Nussbaum, J. L., Roussel, G., Pontarotti, P., Roeckel, N., Mather, I. H., Artzt, K., Lindahl, K. F. and Dautigny, A. (1993). Myelin/oligodendrocyte glycoprotein is a member of a subset of the immunoglobulin superfamily encoded within the major histocompatibility complex. Proceedings of the National Academy of Sciences 90, 7990-7994.

Piljić, A. and Schultz, C. (2006). Annexin A4 Self-Association Modulates General Membrane Protein Mobility in Living Cells. Molecular Biology of the Cell 17, 3318-3328.

Pillai, A. M., Thaxton, C., Pribisko, A. L., Cheng, J.-G., Dupree, J. L. and Bhat, M. A. (2009). Spatiotemporal ablation of myelinating glia-specific neurofascin (Nfasc NF155) in mice reveals gradual loss of paranodal axoglial junctions and concomitant disorganization of axonal domains. Journal of Neuroscience Research 87, 1773-1793.

Poliak, S. and Peles, E. (2003). The local differentiation of myelinated axons at nodes of Ranvier. Nature Reviews. Neuroscience 4, 968-80. 
Polverini, E., Fasano, A., Zito, F., Riccio, P. and Cavatorta, P. (1999). Conformation of bovine myelin basic protein purified with bound lipids. European Biophysics Journal 28, 351-5.

Privat, A., Jacque, C., Bourre, J. M., Dupouey, P. and Baumann, N. (1979). Absence of the major dense line in myelin of the mutant mouse "shiverer". Neuroscience Letters 12, 107-112.

Quarles, R. H. (2007). Myelin-associated glycoprotein (MAG): past, present and beyond. Journal of Neurochemistry 100, 1431-48.

Raff, M. C. (1989). Glial cell diversification in the rat optic nerve. Science 243, $1450-1455$.

Raju, C. S., Go, C., Nord, Y., Hermanson, O., Lo, C., Visa, N. and Castelo-branco, G. (2008). In Cultured Oligodendrocytes the A / B-type hnRNP CBF-A Accompanies MBP mRNA Bound to mRNA Trafficking Sequences. Molecular Biology of the Cell 19, 3008-3019.

Rasband, M. N. (2010a). The axon initial segment and the maintenance of neuronal polarity. Nature Reviews. Neuroscience 11, 552-62.

Rasband, M. N. (2010b). The axon initial segment and the maintenance of neuronal polarity. Nature reviews. Neuroscience 11, 552-62.

Readhead, C., Popko, B., Takahashi, N., Shine, H. D., Saavedra, R. A., Sidman, R. L. and Hood, L. (1987). Expression of a myelin basic protein gene in transgenic shiverer mice: correction of the dysmyelinating phenotype. Cell 48, 703-712.

Ren, S., Scarsdale, J. N., Ariga, T., Zhang, Y., Klein, R. A., Hartmann, R., Kushi, Y., Egge, H. and Yu, R. K. (1993). Mutants of Arabidopsis deficient in the synthesis of alpha-linolenate. Biochemical and genetic characterization of the endoplasmic reticulum linoleoyl desaturase. The Journal of Biological Chemistry 268, 12632-12638.

Richter, R. P., Bérat, R. and Brisson, A. R. (2006). Formation of solid-supported lipid bilayers: an integrated view. Langmuir 22, 3497-3505.

Ridsdale, R. A., Beniac, D. R., Tompkins, T. A., Moscarello, M. A. and Harauz, G. (1997). Three-dimensional structure of myelin basic protein. II. Molecular 
modeling and considerations of predicted structures in multiple sclerosis. The Journal of Biological Chemistry 272, 4269-4275.

Rios, J. C., Rubin, M., St Martin, M., Downey, R. T., Einheber, S., Rosenbluth, J., Levinson, S. R., Bhat, M. and Salzer, J. L. (2003). Paranodal interactions regulate expression of sodium channel subtypes and provide a diffusion barrier for the node of Ranvier. The Journal of Neuroscience 23, 7001-7011.

Roach, A., Boylan, K., Horvath, S., Prusiner, S. B. and Hood, L. E. (1983). Characterization of cloned cDNA representing rat myelin basic protein: absence of expression in brain of shiverer mutant mice. Cell 34, 799-806.

Romero, P., Obradovic, Z., Li, X., Garner, E. C., Brown, C. J. and Dunker, A. K. (2001). Sequence complexity of disordered protein. Proteins 42, 38-48.

Rosen, A., Keenan, K. F., Thelen, M., Nairn, A. C. and Aderem, A. (1990). Activation of protein kinase $\mathrm{C}$ results in the displacement of its myristoylated, alaninerich substrate from punctate structures in macrophage filopodia. The Journal of Experimental Medicine 172, 1211-1215.

Rosenbluth, J. (1980). Central myelin in the mouse mutant shiverer. The Journal of Comparative Neurology 194, 639-48.

Rosenbluth, J., Liang, W. L., Liu, Z., Guo, D. and Schiff, R. (1995). Paranodal structural abnormalities in rat CNS myelin developing in vivo in the presence of implanted O1 hybridoma cells. Journal of Neurocytology 24, 818-824.

Saher, G., Brügger, B., Lappe-Siefke, C., Möbius, W., Tozawa, R.-i., Wehr, M. C., Wieland, F., Ishibashi, S. and Nave, K.-A. (2005). High cholesterol level is essential for myelin membrane growth. Nature Neuroscience 8, 468-475.

Saher, G., Quintes, S. and Nave, K.-A. (2011). Cholesterol: A Novel Regulatory Role in Myelin Formation. The Neuroscientist 17, 79-93.

Saher, G., Rudolphi, F., Corthals, K., Ruhwedel, T., Schmidt, K.-F., Löwel, S., Dibaj, P., Barrette, B., Möbius, W. and Nave, K.-A. (2012). Therapy of PelizaeusMerzbacher disease in mice by feeding a cholesterol-enriched diet. Nature Medicine $18,1130-1136$.

Salzer, J. L. (2003). Polarized domains of myelinated axons. Neuron 40, 297-318. 
Sampo, B., Kaech, S., Kunz, S. and Banker, G. (2003). Membrane Proteins to the Axonal Surface. Neuron 37, 611-624.

Schaeren-Wiemers, N., Bonnet, A., Erb, M., Erne, B., Bartsch, U., Kern, F., Mantei, N., Sherman, D. and Suter, U. (2004). The raft-associated protein MAL is required for maintenance of proper axon-glia interactions in the central nervous system. The Journal of Cell Biology 166, 731-742.

Schuck, S. and Simons, K. (2004). Polarized sorting in epithelial cells: raft clustering and the biogenesis of the apical membrane. Journal of Cell Science 117, 5955-64.

Serrels, A., Timpson, P., Canel, M., Schwarz, J. P., Carragher, N. O., Frame, M. C., Brunton, V. G. and Anderson, K. I. (2009). Real-time study of E-cadherin and membrane dynamics in living animals: implications for disease modeling and drug development. Cancer Research 69, 2714-9.

Shcheprova, Z., Baldi, S., Frei, S. B., Gonnet, G. and Barral, Y. (2008). A mechanism for asymmetric segregation of age during yeast budding. Nature 454, 728-34.

Sherman, D. L. and Brophy, P. J. (2005). Mechanisms of axon ensheathment and myelin growth. Nature Reviews Neuroscience 6, 683-690.

Sherman, D. L., Tait, S., Melrose, S., Johnson, R., Zonta, B., Court, F. a., Macklin, W. B., Meek, S., Smith, A. J. H., Cottrell, D. F. and Brophy, P. J. (2005). Neurofascins are required to establish axonal domains for saltatory conduction. Neuron 48, 737-42.

Shivas, J. M., Morrison, H. a., Bilder, D. and Skop, A. R. (2010). Polarity and endocytosis: reciprocal regulation. Trends in Cell Biology 20, 445-52.

Simmen, T., Höning, S., Icking, A., Tikkanen, R. and Hunziker, W. (2002). AP4 binds basolateral signals and participates in basolateral sorting in epithelial MDCK cells. Nature Cell Biology 4, 154-9.

Simons, M., Kramer, E.-M., Macchi, P., Rathke-Hartlieb, S., Trotter, J., Nave, K.A. and Schulz, J. B. (2002). Overexpression of the myelin proteolipid protein leads to accumulation of cholesterol and proteolipid protein in endosomes/lysosomes: implications for Pelizaeus-Merzbacher disease. The Journal of Cell Biology 157, $327-336$. 
Simons, M., Krämer, E. M., Thiele, C., Stoffel, W. and Trotter, J. (2000). Assembly of myelin by association of proteolipid protein with cholesterol- and galactosylceramide-rich membrane domains. The Journal of Cell Biology 151, $143-54$.

Singer, S. J. and Nicolson, G. L. (1972). The fluid mosaic model of the structure of cell membranes. Science 175, 720-31.

Smith, G. S. T., Seymour, L. V., Boggs, J. M. and Harauz, G. (2012). The 21.5$\mathrm{kDa}$ isoform of myelin basic protein has a non-traditional PY-nuclear-localization signal. Biochemical and Biophysical Research Communications 422, 670-5.

Smith, P. D., Liesegang, G. W., Berger, R. L., Czerlinski, G. and Podolsky, R. J. (1984). A stopped-flow investigation of calcium ion binding by ethylene glycol bis(beta-aminoethyl ether)-N,N'-tetraacetic acid. Analytical Biochemistry 143, 188-195.

Sommer, I. and Schachner, M. (1981). Monoclonal antibodies (O1 to O4) to oligodendrocyte cell surfaces: an immunocytological study in the central nervous system. Developmental Biology 83, 311-327.

Song, A.-H., Wang, D., Chen, G., Li, Y., Luo, J., Duan, S. and Poo, M.-M. (2009). A selective filter for cytoplasmic transport at the axon initial segment. Cell 136, 1148-60.

Spassky, N., Olivier, C., Cobos, I., LeBras, B., Goujet-Zalc, C., Martínez, S., Zalc, B. and Thomas, J. L. (2001). The early steps of oligodendrogenesis: insights from the study of the plp lineage in the brain of chicks and rodents. Developmental Neuroscience 23, 318-326.

Spiegel, I., Adamsky, K., Eshed, Y., Milo, R., Sabanay, H., Sarig-Nadir, O., Horresh, I., Scherer, S. S., Rasband, M. N. and Peles, E. (2007). A central role for Necl4 (SynCAM4) in Schwann cell-axon interaction and myelination. Nature Neuroscience 10, 861-9.

Sulistijo, E. S. and MacKenzie, K. R. (2006). Sequence dependence of BNIP3 transmembrane domain dimerization implicates side-chain hydrogen bonding and a tandem GxxxG motif in specific helix-helix interactions. Journal of Molecular Biology 364, 974-990. 
Suresh, S., Wang, C., Nanekar, R., Kursula, P. and Edwardson, J. M. (2010). Myelin basic protein and myelin protein 2 act synergistically to cause stacking of lipid bilayers. Biochemistry 49, 3456-63.

Swanton, E., Holland, A., High, S. and Woodman, P. (2005). Disease-associated mutations cause premature oligomerization of myelin proteolipid protein in the endoplasmic reticulum. Proceedings of the National Academy of Sciences 102, 4342-4347.

Szuchet, S., Polak, P. E. and Yim, S. H. (1986). Mature oligodendrocytes cultured in the absence of neurons recapitulate the ontogenic development of myelin membranes. Developmental Neuroscience 8, 208-221.

Tait, S., Gunn-Moore, F., Collinson, J. M., Huang, J., Lubetzki, C., Pedraza, L., Sherman, D. L., Colman, D. R. and Brophy, P. J. (2000). An oligodendrocyte cell adhesion molecule at the site of assembly of the paranodal axo-glial junction. The Journal of Cell Biology 150, 657-666.

Takai, Y., Irie, K., Shimizu, K., Sakisaka, T. and Ikeda, W. (2003). Nectins and nectin-like molecules: roles in cell adhesion, migration, and polarization. Cancer Science 94, 655-667.

Teigler, A., Komljenovic, D., Draguhn, A., Gorgas, K. and Just, W. W. (2009). Defects in myelination, paranode organization and Purkinje cell innervation in the ether lipid-deficient mouse cerebellum. Human Molecular Genetics 18, 18971908.

ter Beest, M. B. and Hoekstra, D. (1993). Interaction of myelin basic protein with artificial membranes. Parameters governing binding, aggregation and dissociation. European Journal of Biochemistry / FEBS 211, 689-96.

Tompa, P. (2002). Intrinsically unstructured proteins. Trends in Biochemical Sciences 27, 527-533.

Towbin, H., Staehelin, T. and Gordon, J. (1979). Electrophoretic transfer of proteins from polyacrylamide gels to nitrocellulose sheets: procedure and some applications. Proceedings of the National Academy of Sciences 76, 4350-4354.

Trajkovic, K., Dhaunchak, A. S., Goncalves, J. T., Wenzel, D., Schneider, A., Bunt, G., Nave, K.-A. and Simons, M. (2006). Neuron to glia signaling triggers myelin 
membrane exocytosis from endosomal storage sites. The Journal of Cell Biology 172, 937-48.

Trapp, B. D. and Quarles, R. H. (1982). Presence of the myelin-associated glycoprotein correlates with alterations in the periodicity of peripheral myelin. The Journal of Cell Biology 92, 877-882.

Umemori, H., Kadowaki, Y., Hirosawa, K., Yoshida, Y., Hironaka, K., Okano, H. and Yamamoto, T. (1999). Stimulation of myelin basic protein gene transcription by Fyn tyrosine kinase for myelination. The Journal of Neuroscience 19, 13931397.

Updike, D. L., Hachey, S. J., Kreher, J. and Strome, S. (2011). P granules extend the nuclear pore complex environment in the C. elegans germ line. The Journal of Cell Biology 192, 939-48.

Várnai, P. and Balla, T. (1998). Visualization of phosphoinositides that bind pleckstrin homology domains: calcium- and agonist-induced dynamic changes and relationship to myo-[3H]inositol-labeled phosphoinositide pools. The Journal of Cell Biology 143, 501-510.

Várnai, P., Tóth, B., Tóth, D. J., Hunyady, L. and Balla, T. (2007). Visualization and manipulation of plasma membrane-endoplasmic reticulum contact sites indicates the presence of additional molecular components within the STIM1-Orai1 Complex. The Journal of Biological Chemistry 282, 29678-29690.

Verity, A. N. and Campagnoni, A. T. (1988). Regional expression of myelin protein genes in the developing mouse brain: in situ hybridization studies. Journal of Neuroscience Research 21, 238-248.

Vogel, U. S. and Thompson, R. J. (1988). Molecular structure, localization, and possible functions of the myelin-associated enzyme 2',3'-cyclic nucleotide 3'phosphodiesterase. Journal of Neurochemistry 41, 506-515.

Wang, L. and Boyer, J. L. (2004). The maintenance and generation of membrane polarity in hepatocytes. Hepatology 39, 892-9.

Weber, S. C. and Brangwynne, C. P. (2012). Getting RNA and protein in phase. Cell 149, 1188-91. 
Wegner, M. (2008). A matter of identity: transcriptional control in oligodendrocytes. Journal of Molecular Neuroscience 35, 3-12.

Weimbs, T. and Stoffel, W. (1992). Proteolipid protein (PLP) of CNS myelin: positions of free, disulfide-bonded, and fatty acid thioester-linked cysteine residues and implications for the membrane topology of PLP. Biochemistry 31, 1228912296.

Werner, H. B., Kuhlmann, K., Shen, S., Uecker, M., Schardt, A., Dimova, K., Orfaniotou, F., Dhaunchak, A., Brinkmann, B. G., Möbius, W., Guarente, L., Casaccia-Bonnefil, P., Jahn, O. and Nave, K.-A. (2007). Proteolipid protein is required for transport of sirtuin 2 into CNS myelin. The Journal of Neuroscience 27, 7717-7730.

White, R., Gonsior, C., Bauer, N. M., Kraemer-Albers, E. M., Luhmann, H. J. and Trotter, J. (2011). HnRNP F is a novel component of oligodendroglial RNA transport granules contributing to the regulation of MBP protein synthesis. The Journal of Biological Chemistry 28\%, 1742-1754.

White, R., Gonsior, C., Krämer-Albers, E.-M., Stöhr, N., Hüttelmaier, S. and Trotter, J. (2008). Activation of oligodendroglial Fyn kinase enhances translation of mRNAs transported in hnRNP A2-dependent RNA granules. The Journal of Cell Biology 181, 579-586.

Wight, P. A., Duchala, C. S., Readhead, C. and Macklin, W. B. (1993). A myelin proteolipid protein-LacZ fusion protein is developmentally regulated and targeted to the myelin membrane in transgenic mice. The Journal of Cell Biology 123, $443-454$.

Winckler, B., Forscher, P. and Mellman, I. (1999). A diffusion barrier maintains distribution of membrane proteins in polarized neurons. Nature 397, 698-701.

Winter, J. (1982). Shiverer peripheral myelin contains P2. Nature 298, 471-472.

Wood, D. D. and Moscarello, M. a. (1989). The isolation, characterization, and lipid-aggregating properties of a citrulline containing myelin basic protein. The Journal of Biological Chemistry 264, 5121-7.

Yeung, T., Gilbert, G. E., Shi, J., Silvius, J., Kapus, A. and Grinstein, S. (2008). Membrane phosphatidylserine regulates surface charge and protein localization. Science 319, 210-3. 
Yeung, T., Terebiznik, M., Yu, L., Silvius, J., Abidi, W. M., Philips, M., Levine, T., Kapus, A. and Grinstein, S. (2006). Receptor activation alters inner surface potential during phagocytosis. Science 313, 347-351.

Yin, X., Crawford, T. O., Griffin, J. W., Tu, P. H., Lee, V. M., Li, C., Roder, J. and Trapp, B. D. (1998). Myelin-associated glycoprotein is a myelin signal that modulates the caliber of myelinated axons. The Journal of Neuroscience 18, 1953-1962.

Yool, D. A., Edgar, J. M., Montague, P. and Malcolm, S. (2000). The proteolipid protein gene and myelin disorders in man and animal models. Human Molecular Genetics 9, 987-992.

Yoshikawa, F., Sato, Y., Tohyama, K., Akagi, T., Hashikawa, T., Nagakura-Takagi, Y., Sekine, Y., Morita, N., Baba, H., Suzuki, Y., Sugano, S., Sato, A. and Furuichi, T. (2008). Opalin, a transmembrane sialylglycoprotein located in the central nervous system myelin paranodal loop membrane. The Journal of Biological Chemistry 283, 20830-20840.

Zhou, Q., Choi, G. and Anderson, D. J. (2001). The bHLH transcription factor Olig2 promotes oligodendrocyte differentiation in collaboration with Nkx2.2. Neuron 31, 791-807.

Zuchero, J. B. and Barres, B. a. (2011). Between the sheets: a molecular sieve makes myelin membranes. Developmental Cell 21, 385-6. 


\section{A. Appendix}

Table A.1: List of constructs. Note that antibiotic resistance for pcDNA3.1(-) and pUC vector is ampicillin; and for pEGFPN1 and pSFV4 vector is kanamycin. The numbers stated for the plasmids are the personal internal numbers.

\begin{tabular}{|c|l|l|l|l|}
\hline No. & Plasmid name & Cloning strategy & Enzymes & Vector \\
\hline 008 & $\begin{array}{l}\text { SS-myc- } \\
\text { MAGNter }\end{array}$ & $\begin{array}{l}\text { PCR for MAGNter with } \\
18329 / 18528\end{array}$ & AfeI/HindIII & pcDNA3.1(-) \\
\hline 014 & His-MBP & $\begin{array}{l}\text { PCR for MBP with } \\
19355 / 19363\end{array}$ & BamHI/SpeI & pSFV4 \\
\hline 025 & $\begin{array}{l}\text { SS-myc- } \\
\text { Tmem10Nter }\end{array}$ & $\begin{array}{l}\text { PCR for Tmem10Nter with } \\
18901 / 18902\end{array}$ & AfeI/HindIII & pcDNA3.1(-) \\
\hline 031 & $\begin{array}{l}\text { SS-myc- } \\
\text { MAGNter6(+) }\end{array}$ & $\begin{array}{l}\text { PCR for MAGNter6(-) } \\
\text { with } 18329 / 18991\end{array}$ & AfeI/HindIII & pcDNA3.1(-) \\
\hline 032 & $\begin{array}{l}\text { SS-myc- } \\
\text { MAGNter6(-) }\end{array}$ & $\begin{array}{l}\text { PCR for MAGNter6(-) } \\
\text { with } 18329 / 18990\end{array}$ & AfeI/HindIII & pcDNA3.1(-) \\
\hline 033 & $\begin{array}{l}\text { SS-myc- } \\
\text { Tmem10Nter6(+) }\end{array}$ & $\begin{array}{l}\text { PCR for Tmem10Nter6(+) } \\
\text { with } 18901 / 18993\end{array}$ & AfeI/HindIII & pcDNA3.1(-) \\
\hline 034 & $\begin{array}{l}\text { SS-myc- } \\
\text { Tmem10Nter6(-) }\end{array}$ & $\begin{array}{l}\text { PCR for Tmem10Nter6(-) } \\
\text { with } 18901 / 18992\end{array}$ & AfeI/HindIII & pcDNA3.1(-) \\
\hline 041 & $\begin{array}{l}\text { SS-myc- } \\
\text { MAGNterC10 }\end{array}$ & $\begin{array}{l}\text { PCR for MAGNterC10 } \\
\text { with } 18329 / 19116\end{array}$ & AfeI/HindIII & pcDNA3.1(-) \\
\hline 042 & $\begin{array}{l}\text { SS-myc- } \\
\text { MAGNterC20 }\end{array}$ & $\begin{array}{l}\text { PCR for MAGNterC20 } \\
\text { with } 18329 / 19117\end{array}$ & AfeI/HindIII & pcDNA3.1(-) \\
\hline 043 & $\begin{array}{l}\text { SS-myc- } \\
\text { MAGNterC30 }\end{array}$ & $\begin{array}{l}\text { PCR for MAGNterC30 } \\
\text { with } 18329 / 19118\end{array}$ & $\begin{array}{l}\text { PCR for MAGN/HindIII } \\
\text { with } 18329 / 19121\end{array}$ & pcDNA3.1(-) \\
\hline 046 & $\begin{array}{l}\text { SS-myc- } \\
\text { MAGNterC46 }\end{array}$ & $\begin{array}{l}\text { PCR for Tmem10NterC10 } \\
\text { with } 18901 / 19243\end{array}$ & AfeI/HindIII & pcDNA3.1(-) \\
\hline 051 & $\begin{array}{l}\text { SS-myc- } \\
\text { Tm10NterC10 }\end{array}$ & pcDNA3.1(-) \\
\hline
\end{tabular}


A Appendix

\begin{tabular}{|c|c|c|c|c|}
\hline 052 & $\begin{array}{l}\text { SS-myc- } \\
\text { Tm10NterC20 }\end{array}$ & $\begin{array}{l}\text { PCR for Tmem10NterC20 } \\
\text { with } 18901 / 19244\end{array}$ & AfeI/HindIII & pcDNA3.1(-) \\
\hline 053 & $\begin{array}{l}\text { SS-myc- } \\
\text { Tm10NterC30 }\end{array}$ & $\begin{array}{l}\text { PCR for Tmem10NterC30 } \\
\text { with } 18901 / 19245\end{array}$ & AfeI/HindIII & pcDNA3.1(-) \\
\hline 054 & $\begin{array}{l}\text { SS-myc- } \\
\text { Tm10NterC40 }\end{array}$ & $\begin{array}{l}\text { PCR for Tmem10NterC40 } \\
\text { with } 18901 / 19246\end{array}$ & AfeI/HindIII & pcDNA3.1(-) \\
\hline 055 & $\begin{array}{l}\text { SS-myc- } \\
\text { Tm10NterC50 }\end{array}$ & $\begin{array}{l}\text { PCR for Tmem10NterC50 } \\
\text { with } 18901 / 19247\end{array}$ & AfeI/HindIII & pcDNA3.1(-) \\
\hline 060 & $\begin{array}{l}\text { SS-myc- } \\
\text { Tm10NterC100 }\end{array}$ & $\begin{array}{l}\text { PCR for Tmem10NterC100 } \\
\text { with } 18901 / 19252\end{array}$ & AfeI/HindIII & pcDNA3.1(-) \\
\hline 071 & $\begin{array}{l}\text { SS-myc- } \\
\text { Nfas155NterC10 }\end{array}$ & $\begin{array}{l}\text { PCR for Nfas155NterC10 } \\
\text { with } 18897 / 19364\end{array}$ & AfeI/HindIII & pcDNA3.1(-) \\
\hline 072 & $\begin{array}{l}\text { SS-myc- } \\
\text { Nfas155NterC20 }\end{array}$ & $\begin{array}{l}\text { PCR for Nfas155NterC20 } \\
\text { with } 18897 / 19365\end{array}$ & AfeI/HindIII & pcDNA3.1(-) \\
\hline 073 & $\begin{array}{l}\text { SS-myc- } \\
\text { Nfas155NterC30 }\end{array}$ & $\begin{array}{l}\text { PCR for Nfas155NterC20 } \\
\text { with } 18897 / 19366\end{array}$ & AfeI/HindIII & pcDNA3.1(-) \\
\hline 091 & $\begin{array}{l}\text { SS-myc- } \\
\text { Tm10Nter-HA }\end{array}$ & $\begin{array}{l}\text { PCR for Tmem10Nter-HA } \\
\text { with } 18901 / 20058\end{array}$ & AfeI/HindIII & pcDNA3.1(-) \\
\hline 093 & $\begin{array}{l}\text { SS-myc- } \\
\text { Tm10NterC20- } \\
\text { HA }\end{array}$ & $\begin{array}{l}\text { PCR for Tmem10NterC20- } \\
\text { HA with } 18901 / 20060\end{array}$ & AfeI/HindIII & pcDNA3.1(-) \\
\hline 129 & MBP18-myc & $\begin{array}{lcc}\text { PCR for } & \text { MBP } & \text { with } \\
21768 / 21769 & & \\
\end{array}$ & BamHI/HindIII & pcDNA3.1(-) \\
\hline 130 & MBP18-HA & $\begin{array}{l}\text { PCR for MBP with } \\
21768 / 21770\end{array}$ & BamHI/HindIII & pcDNA3.1(-) \\
\hline 151 & MBP14-Myc & $\begin{array}{l}\text { PCR for MBP with } \\
21768 / 21769\end{array}$ & BamHI/HindIII & pcDNA3.1(-) \\
\hline 155 & $\begin{array}{l}\text { SS-myc-Tm10- } \\
\text { MBP }\end{array}$ & $\begin{array}{l}\text { PCR for MBP with } \\
22951 / 22952\end{array}$ & XhoI/HindIII & pcDNA3.1(-) \\
\hline 171 & $\begin{array}{l}\text { SS-myc-GFP- } \\
\text { Tm10-MBP }\end{array}$ & $\begin{array}{lll}\text { PCR for } & \text { GFP with } \\
24121 / 24122 & & \\
\end{array}$ & NotI/AfeI & pcDNA3.1(-) \\
\hline 172 & $\begin{array}{l}\text { SS-myc-YFP- } \\
\text { Tm10-MBP }\end{array}$ & $\begin{array}{lccc}\text { PCR for } & \text { YFP } & \text { with } \\
24121 / 24122 & & \\
\end{array}$ & NotI/AfeI & pcDNA3.1(-) \\
\hline 181 & $\begin{array}{l}\text { SS-myc-YFP- } \\
\text { Tm10 }\end{array}$ & $\begin{array}{lccc}\text { PCR for } & \text { YFP } & \text { with } \\
24121 / 24122 & & \\
\end{array}$ & NotI/AfeI & pcDNA3.1(-) \\
\hline 195 & $\begin{array}{l}\text { SS-myc-GFP- } \\
\text { Tm10 }\end{array}$ & $\begin{array}{lccc}\text { PCR for } & \text { GFP } & \text { with } \\
24121 / 24122 & & \\
\end{array}$ & NotI/AfeI & pcDNA3.1(-) \\
\hline
\end{tabular}




\begin{tabular}{|c|c|c|c|c|}
\hline 196 & mem- YFP & ordered from clonetech & - & pcDNA \\
\hline 226 & mem-RFP & $\begin{array}{lccc}\text { PCR } & \text { for } & \text { RFP } & \text { with } \\
24809 / 24810 & & \\
\end{array}$ & $\mathrm{XbaI} /$ NotI & pEGFPN1 \\
\hline 239 & MBP 14 & $\begin{array}{l}\text { PCR for MBP with } \\
24986 / 24987\end{array}$ & XhoI/HindIII & pEGFPN1 \\
\hline 262 & $\begin{array}{l}\text { SS-myc-GFP- } \\
\text { Tm10-PH }\end{array}$ & $\begin{array}{l}\text { PCR for PH domain with } \\
25095 / 25096\end{array}$ & XhoI/HindIII & pcDNA3.1(-) \\
\hline 284 & $\begin{array}{l}\text { SS-myc-GFP- } \\
\text { FRB-Tm10- } \\
\text { MBP }\end{array}$ & $\begin{array}{l}\text { PCR for FRB with } \\
25139 / 2540\end{array}$ & AfeI/EcoRI & pcDNA3.1(-) \\
\hline 285 & $\begin{array}{l}\text { SS-myc- } \\
\text { Dendra2-Tm10- } \\
\text { MBP }\end{array}$ & Subcloned Dendra2 & NotI/AfeI & pcDNA3.1(-) \\
\hline 290 & $\begin{array}{l}\text { SS-myc-GFP- } \\
\text { BNIP TM-MBP }\end{array}$ & $\begin{array}{l}\text { BNIP TM by annealing of } \\
25188 / 25189\end{array}$ & AfeI/XhoI & pcDNA3.1(-) \\
\hline 302 & $\begin{array}{l}\text { SS-myc-GFP- } \\
\text { BNIP TM }\end{array}$ & $\begin{array}{l}\text { BNIP TM by annealing of } \\
25188 / 25189\end{array}$ & AfeI/XhoI & pcDNA3.1(-) \\
\hline 315 & $\begin{array}{l}\text { SS-myc-GFP- } \\
\text { BNIP TM-PH }\end{array}$ & $\begin{array}{l}\text { BNIP TM by annealing of } \\
25188 / 25189\end{array}$ & AfeI/XhoI & pcDNA 3.1 \\
\hline 324 & $\begin{array}{l}\text { SS-myc-GFP- } \\
\text { GyPTM }\end{array}$ & $\begin{array}{l}\text { GyPTM by annealing } \\
\text { bm01/bm02 }\end{array}$ & AfeI/XhoI & pcDNA3.1(-) \\
\hline 325 & $\begin{array}{l}\text { SS-myc-GFP- } \\
\text { GyPTM-MBP }\end{array}$ & $\begin{array}{l}\text { GyPTM by annealing } \\
\text { bm01/bm02 }\end{array}$ & AfeI/XhoI & pcDNA3.1(-) \\
\hline 328 & $\begin{array}{l}\text { SS-myc-GFP- } \\
\text { GyPTM-PH }\end{array}$ & $\begin{array}{l}\text { GyPTM by annealing } \\
\text { bm01/bm02 }\end{array}$ & AfeI/XhoI & pcDNA3.1(-) \\
\hline 336 & $\begin{array}{l}\text { SS-myc-FKBP- } \\
\text { Tm10-mCherry }\end{array}$ & $\begin{array}{l}\text { PCR for FKBP with } \\
25143 / 25144\end{array}$ & NotI/AfeI & pcDNA3.1(-) \\
\hline 338 & $\begin{array}{l}\text { SS-myc- } \\
\text { Dendra2-Tm10 }\end{array}$ & Subcloned Dendra2 & NotI/AfeI & pcDNA3.1(-) \\
\hline 344 & $\begin{array}{l}\text { myc } \\
\text { tmem10nterc20ha } \\
\text { MBP }\end{array}$ & Subcloned MBP & XhoI/HindIII & pcDNA3.1(-) \\
\hline 347 & $\begin{array}{l}\text { SS-myc- } \\
\text { mCherry-Tm10- } \\
\text { MBP }\end{array}$ & $\begin{array}{l}\text { PCR for mCherry with } \\
25924 / 25925\end{array}$ & NotI/AfeI & pcDNA3.1(-) \\
\hline 355 & $\begin{array}{l}\text { SS-myc-GFP- } \\
\text { PLPTM4-MBP }\end{array}$ & $\begin{array}{l}\text { PCR for PLPTM4 with } \\
26184 \text { and } 26185\end{array}$ & AfeI/XhoI & pcDNA3.1(-) \\
\hline
\end{tabular}




\begin{tabular}{|c|c|c|c|c|}
\hline 356 & $\begin{array}{l}\text { SS-myc-GFP- } \\
\text { PLPTM4 }\end{array}$ & $\begin{array}{l}\text { PCR for PLPTM4 with } \\
26184 \text { and } 26185\end{array}$ & AfeI/XhoI & pcDNA3.1(-) \\
\hline 357 & $\begin{array}{l}\text { SS-myc- } \\
\text { mCherry- } \\
\text { PLPTM4-MBP }\end{array}$ & $\begin{array}{l}\text { PCR for PLPTM4 with } \\
26184 \text { and } 26185\end{array}$ & AfeI/XhoI & pcDNA3.1(-) \\
\hline 358 & $\begin{array}{l}\text { SS-myc- } \\
\text { mCherry-Tm10- } \\
\text { PH }\end{array}$ & Subcloned PH domain & XhoI/HindIII & pcDNA3.1(-) \\
\hline 363 & $\begin{array}{l}\text { SS-myc-GFP- } \\
\text { MAGNterC46 }\end{array}$ & $\begin{array}{l}\text { PCR for MAGNterC46 } \\
\text { with } 26318 / 26319\end{array}$ & AfeI/HindIII & pcDNA3.1(-) \\
\hline 364 & $\begin{array}{l}\text { SS-myc-GFP- } \\
\text { Tmem10NterC50 }\end{array}$ & $\begin{array}{l}\text { PCR for Tmem10NterC50 } \\
\text { with } 26320 / 26321\end{array}$ & AfeI/HindIII & pcDNA3.1(-) \\
\hline 368 & $\begin{array}{l}\text { SS-myc- } \\
\text { mCherry- } \\
\text { Tm10Nter }\end{array}$ & $\begin{array}{l}\text { PCR for mCherry with } \\
25924 / 25925\end{array}$ & NotI/AfeI & pcDNA3.1(-) \\
\hline 369 & $\begin{array}{l}\text { SS-myc- } \\
\text { mCherry- } \\
\text { Tm10NterC10 }\end{array}$ & $\begin{array}{l}\text { PCR for mCherry with } \\
25924 / 25925\end{array}$ & NotI/AfeI & pcDNA3.1(-) \\
\hline 370 & $\begin{array}{l}\text { SS-myc- } \\
\text { mCherry- } \\
\text { Tm10NterC20 }\end{array}$ & $\begin{array}{l}\text { PCR for mCherry with } \\
25924 / 25925\end{array}$ & NotI/AfeI & pcDNA3.1(-) \\
\hline 371 & $\begin{array}{l}\text { SS-myc- } \\
\text { mCherry- } \\
\text { Tm10NterC30 }\end{array}$ & $\begin{array}{l}\text { PCR for mCherry with } \\
25924 / 25925\end{array}$ & NotI/AfeI & pcDNA3.1(-) \\
\hline 372 & $\begin{array}{l}\text { SS-myc- } \\
\text { mCherry- } \\
\text { Tm10NterC40 }\end{array}$ & $\begin{array}{l}\text { PCR for mCherry with } \\
25924 / 25925\end{array}$ & NotI/AfeI & pcDNA3.1(-) \\
\hline 380 & $\begin{array}{l}\text { SS-myc- } \\
\text { mCherry- } \\
\text { PLPTM4-MBP }\end{array}$ & $\begin{array}{l}\text { PCR for PLPTM4 with } \\
26184 \text { and } 26185\end{array}$ & AfeI/XhoI & pcDNA3.1(-) \\
\hline 409 & $\begin{array}{l}\text { SS-myc- } \\
\text { mCherry- } \\
\text { GyPTM }\end{array}$ & $\begin{array}{l}\text { GyPTM by annealing } \\
\text { bm01/bm02 }\end{array}$ & AfeI/XhoI & pcDNA3.1(-) \\
\hline 410 & $\begin{array}{l}\text { SS-myc- } \\
\text { mCherry- } \\
\text { GyPTM-MBP }\end{array}$ & $\begin{array}{l}\text { GyPTM by annealing } \\
\text { bm01/bm02 }\end{array}$ & AfeI/XhoI & pcDNA3.1(-) \\
\hline 421 & MBP FtoS & ordered from genescript & - & $\mathrm{pUC}$ \\
\hline
\end{tabular}




\begin{tabular}{|c|c|c|c|c|}
\hline 422 & $\begin{array}{l}\text { SS-myc- } \\
\text { mCherry- } \\
\text { GyPTM-MBP } \\
\text { FtoS }\end{array}$ & $\begin{array}{l}\text { PCR for MBP FtoS with } \\
21768 / 21770\end{array}$ & BamHI/HindIII & pcDNA3.1(-) \\
\hline 423 & $\begin{array}{l}\text { SS-myc-GFP- } \\
\text { Tm10-MBP } \\
\text { FtoS }\end{array}$ & $\begin{array}{l}\text { PCR for MBP FtoS with } \\
22951 / 22952\end{array}$ & XhoI/HindIII & pcDNA3.1(-) \\
\hline 424 & MBP FtoS-HA & $\begin{array}{l}\text { PCR for MBP FtoS with } \\
21768 / 21770\end{array}$ & BamHI/HindIII & pcDNA3.1(-) \\
\hline 425 & $\begin{array}{l}\text { SS-myc- } \\
\text { mCherry- } \\
\text { PLPTM4-MBP } \\
\text { FtoS }\end{array}$ & $\begin{array}{l}\text { PCR for MBP FtoS with } \\
21768 / 21770\end{array}$ & BamHI/HindIII & pcDNA3.1(-) \\
\hline 426 & His-MBP FtoS & $\begin{array}{l}\text { PCR for MBP } \\
\text { with19355/19363 }\end{array}$ & BamHI/SpeI & pSFV4 \\
\hline 442 & MBP FtoY & ordered from genescript & - & $\mathrm{pUC}$ \\
\hline 443 & MBP FtoA & ordered from genescript & - & $\mathrm{pUC}$ \\
\hline 444 & MBP FtoI & ordered from genescript & - & $\mathrm{pUC}$ \\
\hline 445 & $\begin{array}{l}\text { SS-myc- } \\
\text { mCherry- } \\
\text { GyPTM-MBP } \\
\text { FtoY }\end{array}$ & $\begin{array}{l}\text { PCR for MBP FtoY with } \\
21768 / 21770\end{array}$ & BamHI/HindIII & pcDNA3.1(-) \\
\hline 446 & $\begin{array}{l}\text { SS-myc- } \\
\text { mCherry- } \\
\text { GyPTM-MBP } \\
\text { FtoA }\end{array}$ & $\begin{array}{l}\text { PCR for MBP FtoA with } \\
21768 / 21770\end{array}$ & BamHI/HindIII & pcDNA3.1(-) \\
\hline 447 & $\begin{array}{l}\text { SS-myc- } \\
\text { mCherry- } \\
\text { GyPTM-MBP } \\
\text { FtoI }\end{array}$ & $\begin{array}{l}\text { PCR for MBP FtoA with } \\
21768 / 21770\end{array}$ & BamHI/HindIII & pcDNA3.1(-) \\
\hline
\end{tabular}


Table A.2: List of primers.

\begin{tabular}{|c|c|}
\hline $\begin{array}{l}\text { Internal } \\
\text { no. }\end{array}$ & Sequence $\left(5^{\prime}-3^{\prime}\right)$ \\
\hline 18329 & AAAAAAAAAAGCGCTGTGGGCGTGGGGGGC \\
\hline 18528 & TTTTTTTTTAAGCTTTCACGTCTGGGTGATGTAGCACACAATG \\
\hline 18897 & AAAAAAAAAAGCGCTATGGCCAGGCAGCAG \\
\hline 18898 & TTTTTTTTTAAGCTTTCAGGCCAGGGAATAGATG \\
\hline 18899 & TTTTTTTTTAAGCTTTCACTTGATGAAGCAGACGATCAG \\
\hline 18901 & AAAAAAAAAAGCGCTATGAGTTTTTCACTGAACTTCAC \\
\hline 18902 & TTTTTTTTTAAGCTTTCATCTTCTTCGGTGAATCAAAGTAAATAG \\
\hline 18903 & TTTTTTTTTTCAGTTATCTAGATCCGGTGG \\
\hline 18990 & $\begin{array}{l}\text { TTTTTTTTTAAGCTTTCATCTTCTTCTTCTTCTTCTTCCTCCTCCTCCC } \\
\text { GTCTGGGTGATGTAGC }\end{array}$ \\
\hline 18991 & $\begin{array}{l}\text { TTTTTTTTTAAGCTTTCAGTCGTCGTCGTCGTCGTCTCCTCCTCCTCC } \\
\text { CGTCTGGGTGATGTAGC }\end{array}$ \\
\hline 18992 & $\begin{array}{l}\text { TTTTTTTTTAAGCTTTCAGTCGTCGTCGTCGTCGTCTCCTCCTCCTCC } \\
\text { TCTTCTTCGGTGAATCAAAG }\end{array}$ \\
\hline 18993 & $\begin{array}{l}\text { TTTTTTTTTAAGCTTTCATCTTCTTCTTCTTCTTCTTCCTCCTCCTCCI } \\
\text { CTTCTTCGGTGAATCAAAG }\end{array}$ \\
\hline 19116 & TTTTTTTTTAAGCTTTCAGGAGCTCTCCGTGAC \\
\hline 19117 & TTTTTTTTTAAGCTTTCAGACATGAGGGTTGTCTCC \\
\hline 19118 & TTTTTTTTTAAGCTTTCACCCAGAGATTCTGAATTCGG \\
\hline 19121 & TTTTTTTTTAAGCTTTCAGTGACAATCCCGGG \\
\hline 19243 & TTTTTTTTTAAGCTTTCACATGGCCTCAATGCTG \\
\hline 19244 & TTTTTTTTTAAGCTTTCATGAAATTTCACATGGTCTGTC \\
\hline 19245 & TTTTTTTTTAAGCTTTCACTCAGATATCTTGGGATTGTC \\
\hline 19246 & TTTTTTTTTAAGCTTTCACTTCTCATGTGTGGGTGATC \\
\hline 19247 & TTTTTTTTTAAGCTTTCATATGTGGGCCTCTTGTG \\
\hline 19252 & TTTTTTTTTAAGCTTTCAGGCCAGGACCACTTTG \\
\hline 19355 & AAAAAAAAAGGATCCATGGCATCACAGAAGAGAC \\
\hline 19356 & TTTTTTTTTAAGCTTTCAGCGTCTCGCCATG \\
\hline 19363 & TTTTTTTTTACTAGTGCGTCTCGCCATGG \\
\hline 19364 & TTTTTTTTTAAGCTTTCATGGGTACTTGCCGCC \\
\hline 19365 & TTTTTTTTTAAGCTTTCAGCCCAAGGGGACATCC \\
\hline 19366 & TTTTTTTTTAAGCTTTCATGAACCATCTTCTTCTTTGGG \\
\hline 20058 & $\begin{array}{l}\text { TTTAAGCTTTCACTCGAGAGCGTAATCTGGAACATCGTATGGGTATC } \\
\text { CTCCTCCTCTTCTTCGGTGAATCAAAG }\end{array}$ \\
\hline
\end{tabular}




\begin{tabular}{|c|c|}
\hline 20060 & $\begin{array}{l}\text { TTTAAGCTTTCACTCGAGAGCGTAATCTGGAACATCGTATGGGTATC } \\
\text { CTCCTCCTGAAATTTCACATGGTCTG }\end{array}$ \\
\hline 21768 & AAAAAAAGGATCCACCATGGCATCACAGAAGAGAC \\
\hline 21769 & $\begin{array}{l}\text { TTTTTTAAGCTTTCACAGATCCTCTTCAGAGATGAGTTTCTGCTCTC } \\
\text { CTCCTCCGCGTCTCGCCATGG }\end{array}$ \\
\hline 21770 & $\begin{array}{l}\text { TTTTTTAAGCTTTCAAGCGTAATCTGGAACATCGTATGGGTATCCTC } \\
\text { CTCCGCGTCTCGCCATGG }\end{array}$ \\
\hline 22951 & AAAAAACTCGAGGGAGGAGGAGGAATGGCATCACAGAAGAGACC \\
\hline 22952 & TTTTTTAAGCTTTCAGCGTCTCGCCATGGG \\
\hline 24121 & $\begin{array}{l}\text { AAAAAAAGCGGCCGCGGAGGAGGAGGAATGGTGAGCAAGGGCGA } \\
\text { GG }\end{array}$ \\
\hline 24122 & TTTTTTAGCGCTCTTGTACAGCTCGTCCATGCCG \\
\hline 24809 & AAAAAATCTAGAGCCTCCTCCGAGGAC \\
\hline 24810 & TTTTTTGCGGCCGCTTAGGCGCCGGTGGAG \\
\hline 24986 & AAAAAACTCGAGACCATGGCATCACAGAAGAGACC \\
\hline 24987 & TTTTTTAAGCTTGCGTCTCGCCATGGG \\
\hline 25095 & AAAAAACTCGAGGCCCTTCTGAAGGGCAG \\
\hline 25096 & TTTTTTAAGCTTTCAGTGGATGATCTTGCGCAGG \\
\hline 25139 & AAAAAAGCGCTGGAGGAGGAGGAATCCTCTGGCATGAGATGTGG \\
\hline 25140 & TTTTTGAATTCTCCTCCTCCTCCCTTTGAGATTCGTCGGAACACATG \\
\hline 25143 & AAAAAAAGCGGCCGCGGAGGAGGAGGAGTGCAGGTGGAAACCATC \\
\hline 25144 & $\begin{array}{l}\text { TTTTTTAGCGCTTCCTCCTCCTCCTTCCAGTTTTAGAAGCTCCACAT } \\
\text { CG }\end{array}$ \\
\hline 25188 & $\begin{array}{l}\text { GCTCTTAAAGTTTTTCTTCCTTCTCTTCTTCTTTCTCATCTTCTTCTT } \\
\text { ATTGGTCTTGGTATTTATATTGGTCGTCGTC }\end{array}$ \\
\hline 25189 & $\begin{array}{l}\text { TCGAGACGACGACCAATATAAATACCAAGACCAATAAGAAGAAGAT } \\
\text { GAGAAAGAAGAAGAGAAGGAAGAAAAACTTTAAGAGC }\end{array}$ \\
\hline 25829 & $\begin{array}{l}\text { AAAACTCGAGGGAGGAGGAGGATTTCTGAACTGTTGCCCGGGCTGC } \\
\text { TGTATGGAACCCGGAGGAGGAGTGAGCAAGGGCGAG }\end{array}$ \\
\hline 25830 & $\begin{array}{l}\text { TTTTTTAAGCTTTCATCTAGAGCAGGTCTTCTTCTTGTACAGCTCGTC } \\
\text { CATG }\end{array}$ \\
\hline 25924 & AAAAAAGCGGCCGCGTGAGCAAGGGCGAG \\
\hline 25925 & TTTTTTAGCGCTTCCTCCTCCCTTGTACAGCTCGTCCATG \\
\hline 26184 & AAAAAAAGCGCTGGAGGAGGAGGATTCCAAATGACCTTCCACCTG \\
\hline 26185 & $\begin{array}{l}\text { TTTTTTCTCGAGGCCGCCGCCAGTGGCAGCAATCATGAAGGTGAGC } \\
\text { AG }\end{array}$ \\
\hline 26318 & AAAAAAAGCGCTGGGGGCCACTGGGG \\
\hline
\end{tabular}




\begin{tabular}{|l|l|}
\hline 26319 & TTTTTTAAGCTTTCATCTAGAGTGACAATCCCGGGTAGAGAC \\
\hline 26320 & AAAAAAAGCGCTATGAGTTTTTCACTGAACTTCACCC \\
\hline 26321 & TTTTTTAAGCTTTCATCTAGATATGTGGGCCTCTTGTGCTC \\
\hline bm01 & $\begin{array}{l}\text { GCTCCAGAGATAACACTCATTATTTTTCTGGTGATGGCTGCTGTTATTG } \\
\text { GAACGATCCTCTTAATTTCTTACGGTATTCGCCGAC }\end{array}$ \\
\hline bm02 & $\begin{array}{l}\text { TCGAGTCGGCGAATACCGTAAGAAATTAAGAGGATCGTTCCAATAACA } \\
\text { GCAGCCATCACCAGAAAAATAATGAGTGTTATCTCTGGAGC }\end{array}$ \\
\hline
\end{tabular}


"At times our own light goes out and is rekindled by a spark from another person. Each of us has cause to think with deep gratitude of those who have lighted the flame within us."

Albert Schweitzer

\section{Acknowledgements}

My first and foremost thanks goes to my supervisor Prof. Mikael Simons for giving me a chance to work in his lab. Thanks Mika! You always guided me with great enthusiasm. Our scientific discussions and the numerous times I asked you 'Do you have few minutes' would go a long way down the memory lane. You taught me the strength of good ideas in scientific research.

I sincerely thank my committee members: Prof. Peter Rehling and Prof. Dirk Görlich for their scientific insights and friendly discussions during thesis committee meetings. Thank you Dirk for all the valuable advices and very helpful collaborations! Your pertinent questioning taught me the importance of the clarity in scientific thoughts and presentations. It was a great pleasure to discuss science with you.

Many thanks to Dr. Steffen Frey for a lot of intellectual and technical inputs into the project on several occasions. Steffen, for me you are not just a collaborator, but a researcher with whom I never had to think twice before asking a question. I will always remember the promptness with which you replied to all my long emails that were filled with a bunch of questions. Your inputs were of great help in moving the project forward in crucial times.

I would also like to thank Nicolas Snaidero for all the EM experiments. Thanks a lot Nic for sometimes doing the experiments on very short notices! I thank Gesa Pähler for taking out time during her thesis writing and helping with the FTIR experiments. I thank Giselheid Schulz and Kirsten Fladung for excellent technical help. Many thanks to Basti and Lena for meticulously correcting parts of my thesis; Nic for his feedback on introduction, and Mayur for doing last-minute proof-reading. A 
very special thanks to Harish for teaching me $\mathrm{AT}_{\mathrm{E} X} \mathrm{X}$ and all the help with formating.

Special thanks to my enthusiastic and motivated lab rotation students: Christia Reetz and and Isabel Ohs. Tina, I would always cherish our friendship. I truly enjoyed our chatting session whether it was right across the bench or sitting in the cafeteria. Thanks for all the personal and scientific support!

The life in lab was full of fun in the last three years and the credit goes to all the former and current members of AG Simons: Basti, Tina, Shima, Marie, Nils, Schanila, Chieh, Larisa, Marieka, Holger, Katrin, Aniket and Anja. Mosi, Natalia, Lena and Nic: you are all great colleagues and friends. You guys influenced me not just scientifically, but also in personal life. I would cherish the memories of mensa time, coffee time and not to forget the small tea sessions. Thanks Mosi and Natalia for sharing the fun and frustration, specially during thesis writing.

People outside the lab also contributed indirectly to this thesis a lot. Bro and Sravanti, you gave me a 'home' away from home and made me stand strong through all the storms. Thanks for being always there! I would keep going ahead with a feeling of security that you both are right behind.

I thank Mayur, Ishwar and Dada for their friendship, right from the very early days in Göttingen and also many other friends: Nadya, Gopi, Vimal, Atul Bharde, Srinivas, Swati, Prem and Smriti, Gopal and Saujanya, Atul and Miraj, Vaibhav bhaiya, Varun, Sarat, Shalaka, Pawan, Tanmoy, Somu, Sonia, Anjali, Heena and many more.

All these last five years would not have been the same without the support and care from one person. Thank you Harish! You have been my greatest pillar of strength, both in personal and professional life. From tolerating my blabbering sessions to fixing every small tiny problem in my life, I thank you for the wonderful journey.

I am grateful to my parents, grand parents and my sister for giving me unconditional love, for believing in me and supporting me in chasing my dreams. You are all my source of inspiration for whatever I have done or will do in my life. 


\section{Curriculum Vitae}

\section{Personal details}

$\begin{array}{ll}\text { Name } & \text { Shweta Aggarwal } \\ \text { Date of birth } & 10.03 .1984 \\ \text { Place of birth } & \text { Jalandhar, India } \\ \text { Nationality } & \text { Indian } \\ \text { Email address } & \text { shwetaagg@gmail.com }\end{array}$

\section{Languages}

$\begin{array}{ll}\text { English } & \text { fluent } \\ \text { German } & \text { beginners level } \\ \text { Hindi } & \text { mother tongue }\end{array}$

\section{Studies}

2009- 2012 PhD in the Department of Cellular Neuroscience Prof. Mikael Simons, Max Planck Institute of Experimental Medicine, Göttingen, Germany

2008-2009 Research project at European Neuroscience Institute Dr. Silvio Rizzoli, Göttingen, Germany

2007-2008Ｍ.Sc./PhD Molecular Biology Program International Max Planck Research School, Göttingen, Germany, Grade: 1.35 (A) 
2005 -2007 M.Sc (Biotechnology)

Jawaharlal Nehru University, Delhi, India, Grade: 8.31/9 (A)

2002-2005 B.Sc (Hons.), Biochemistry

Daulat Ram College, University of Delhi, India, Marks: 80\% (A)

1999-2001 Higher Secondary School

D.A.V Senior Secondary School, Noida, India, Marks: 83\% (A)

\section{Scholarships}

2007-2008 International Max Planck Research School Stipend

2005-2007 Department of Biotechnology (DBT) sponsored scholarship

\section{Research Experience}

2009- 2012 PhD thesis at Max Planck Institute of Experimental Medicine Prof. Mikael Simons, Göttingen, Germany.

Title: Mechanisms of lipid-rich myelin membrane sheet formation

2008-2009 Research project at European Neuroscience Institute

Dr. Silvio Rizzoli, Göttingen, Germany

Title: Molecular determinants of synaptic vesicle formation

$2007-2008$

Lab rotation project at Max Planck Institute for Biophysical Chemistry

Prof. Reinhard Jahn, Göttingen, Germany

Title: Characterization of early endosomal sorting in vitro

2007-2008 Lab rotation project at European Neuroscience Institute

Dr. Silvio Rizzoli, Göttingen, Germany 
Title: STED microscopy reveals different patterns of transferrin recycling in different cell types

2007-2008 Lab rotation project at European Neuroscience Institute

Dr. Stefan Eimer, Göttingen, Germany

Title: Functional characterization of Ric-19 in C.elegans

2006-2007 Lab rotation project at Jawaharlal Nehru University

Dr. K. J. Mukherjee, Delhi, India

Title: Bioprocess and genetic strategies to generate quiescent cells

for high level recombinant protein production

\section{Teaching}

\section{Tutor of the lectures}

2009 - 2010 "Protein Sorting and Processing", "Vesicular Transport" and "Organelle Biosynthesis" within the International $\mathrm{MSc} / \mathrm{PhD}$ programs "Molecular Biology" and "Neurosciences"

\section{Supervisor of lab rotations}

2009 - 2011 Christina Reetz within the International MSc/PhD Program "Neuroscience"

Isabel Ohs within MSc "Molecular Medicine" Program

\section{Publications}

- "Cell polarity in myelinating glia: From membrane flow to diffusion barriers" Mikael Simons, Nicolas Snaidero, Shweta Aggarwal, Biochimica et Biophysica Acta, 1821, 1146-1153, January 2012.

- "Self-seggregation of myelin membrane lipids in model membranes" Larisa Yurlova, Nicoletta Kahya, Shweta Aggarwal, Hermann-Josef Kaiser, Salvatore Chiantia, Mostafa Bakhti, Yael Pewzner-Jung, Oshrit Ben-David, Anthony H. Futerman, Britta Brügger, Mikael Simons, Biophysical Journal, 101, 2713-2720, December 2011. 
- "Central nervous system myelin: structure, synthesis and assembly" Shweta Aggarwal, Larisa Yurlova, Mikael Simons, Trends in Cell Biology, Vol. 21, No. 10, 585-593, October 2011.

- "A size barrier limits protein diffusion at the cell surface to generate lipidrich myelin-membrane sheets" Shweta Aggarwal, Larisa Yurlova, Nicolas Snaidero, Christina Reetz, Steffen Frey, Johannes Zimmermann, Gesa Pähler, Andreas Janshoff, Jens Friedrichs, Daniel J. Müller, Cornelia Goebel, Mikael Simons, Developmental Cell, 21, 445-456, September 2011.

- "Sorting in early endosomes reveals connections to docking- and fusion-associated factors" Sina V. Barysch, Shweta Aggarwal, Reinhard Jahn, Silvio O.Rizzoli, PNAS, Vol. 106, No. 24, 9697-9702, June 2009.

\section{Posters}

- "A molecular sieve that generates a lipid-rich insulator" Shweta Aggarwal, Larisa Yurlova, Nicolas Snaidero, Christina Reetz, Steffen Frey, Mikael Simons, EMBO, Nice (2012).

- "A molecular sieve that generates a lipid-rich insulator" Shweta Aggarwal, Larisa Yurlova, Nicolas Snaidero, Christina Reetz, Steffen Frey, Mikael Simons, IMPRS, Molecular Biology Program Evaluation, Göttingen (2012).

- "A molecular sieve generates lipid-rich myelin-membrane sheets" Shweta Aggarwal, Larisa Yurlova, Nicolas Snaidero, Christina Reetz, Steffen Frey, Mikael Simons, Scientific Advisory Board Meeting, Göttingen (2011).

- "A molecular sieve generates lipid-rich myelin-membrane sheets" Shweta Aggarwal, Larisa Yurlova, Nicolas Snaidero, Christina Reetz, Steffen Frey, Mikael Simons, GGNB Science Day, Göttingen (2011).

- "A size barrier limits protein diffusion at the cell surface to generate lipid rich myelin-membrane sheets" Shweta Aggarwal, Larisa Yurlova, Nicolas Snaidero, Christina Reetz, Steffen Frey, Mikael Simons, Neurizons, Göttingen (2011).

- "Myelin membrane trafficking and expansion in oligodendrocytes. Horizons in Molecular Biology" Larisa Yurlova, Shweta Aggarwal, Johannes Zimmermann, Mikael Simons, Horizons in Molecular Biology, Göttingen (2009). 
- "Sorting in early endosomes reveals connections to docking- and fusion- associated factors"' Sina V. Barysch, Shweta Aggarwal, Reinhard Jahn, Silvio Rizzoli, Horizons in Molecular Biology, Göttingen (2009).

\section{Talks}

- "A molecular sieve that generates a lipid-rich insulator" IMPRS, Molecular Biology Program Evaluation, Göttingen (2012).

- "Molecular Mechanisms of myelin membrane assembly" Joint Symposium, Göttingen (2010).

\section{Extracurricular Activities}

\section{Co-organizer}

2009

$6^{\text {th }}$ International PhD Student Symposium: "Horizons in Molecular Biology" Göttingen, Germany. 